\title{
Perinatal inflammation and adverse outcomes of the intestine and liver
}

Citation for published version (APA):

Heymans, C. (2022). Perinatal inflammation and adverse outcomes of the intestine and liver. [Doctoral Thesis, Maastricht University]. ProefschriftMaken. https://doi.org/10.26481/dis.20220204ch

Document status and date:

Published: 01/01/2022

DOI:

10.26481/dis.20220204ch

Document Version:

Publisher's PDF, also known as Version of record

\section{Please check the document version of this publication:}

- A submitted manuscript is the version of the article upon submission and before peer-review. There can be important differences between the submitted version and the official published version of record.

People interested in the research are advised to contact the author for the final version of the publication, or visit the DOI to the publisher's website.

- The final author version and the galley proof are versions of the publication after peer review.

- The final published version features the final layout of the paper including the volume, issue and page numbers.

Link to publication

\footnotetext{
General rights rights.

- You may freely distribute the URL identifying the publication in the public portal. please follow below link for the End User Agreement:

www.umlib.nl/taverne-license

Take down policy

If you believe that this document breaches copyright please contact us at:

repository@maastrichtuniversity.nl

providing details and we will investigate your claim.
}

Copyright and moral rights for the publications made accessible in the public portal are retained by the authors and/or other copyright owners and it is a condition of accessing publications that users recognise and abide by the legal requirements associated with these

- Users may download and print one copy of any publication from the public portal for the purpose of private study or research.

- You may not further distribute the material or use it for any profit-making activity or commercial gain

If the publication is distributed under the terms of Article $25 \mathrm{fa}$ of the Dutch Copyright Act, indicated by the "Taverne" license above, 


\section{Perinatal Inflammation and Adverse}

Outcomes of the Intestine and Liver

Cathelijne Heymans 
(c) Cathelijne Heymans, Maastricht 2021

All rights reserved. No parts of this thesis may be reproduced or distributed in any form or by any means, without prior permission in writing by the author, or when appropriate, by the publisher.

Cover design: Famoose by Frank Mooren | www.famoose.be

Layout: ProefschriftMaken | www.proefschriftmaken.nl

Printed by: ProefschriftMaken | www.proefschriftmaken.nl

ISBN: 978-94-6423-553-1 


\title{
Perinatal Inflammation and Adverse Outcomes of the Intestine and Liver
}

\author{
PROEFSCHRIFT \\ ter verkrijging van de graad van doctor aan de Universiteit Maastricht, \\ op gezag van de Rector Magnificus, Prof. Dr. Rianne M. Letschert \\ volgens het besluit van het College van Decanen, \\ in het openbaar te verdedigen \\ op vrijdag 4 februari 2022 om 12:00 uur
} door 


\section{Promotoren}

Prof. Dr. Wim G. van Gemert

Dr. Tim G.A.M. Wolfs

\section{Copromotor}

Dr. Kaatje Lenaerts

\section{Beoordelingscommissie}

Prof. Dr. Steven W.M. Olde Damink (voorzitter)

Prof. Dr. Jan B.F. Hulscher (UMCG)

Prof. Dr. Daisy M.A.E. Jonkers

Prof. Dr. Laurents P.S. Stassen

Dr. Joep P.M. Derikx (Amsterdam UMC) 


\section{Table of contents}

CHAPTER 1 General introduction and outline of this thesis

CHAPTER 2 Chronic intra-uterine Ureaplasma parvum infection induces injury of the enteric nervous system in ovine fetuses

Frontiers in Immunology. 2020. 11(189)

CHAPTER 3 Chorioamnionitis induces enteric nervous system injury: effects of timing and inflammation in the ovine fetus

Molecular Medicine. 2020. 26(1)

CHAPTER 4 Alterations of the enteric nervous system and intestinal motility in a mouse necrotizing enterocolitis model

CHAPTER 5 Chorioamnionitis induces hepatic inflammation and timedependent changes of the enterohepatic circulation in the ovine fetus 89 Scientific Reports. 2021. 11(1)

CHAPTER 6 Prophylactic intra-uterine $\beta$-cyclodextrin administration during intra-uterine Ureaplasma parvum infection partly prevents liver inflammation without interfering with the enterohepatic circulation of the fetal sheep

Nutrients. 2020. 12(5)

CHAPTER 7 General discussion, implications and future perspectives

CHAPTER 8 Summary

Samenvatting

CHAPTER 9 Impact

APPENDICES Abbreviations 

General introduction and outline of this thesis 



\section{General Introduction}

\section{Preterm birth}

The normal duration of a pregnancy is 37 to 42 weeks. Birth between 22 and 37 weeks of gestation is defined as premature birth (1). At this time-point, the child is still immature and therefore needs extra medical care. Premature birth is the most important cause of neonatal morbidity and mortality around birth (2). It is estimated that one in every ten births worldwide are preterm accounting for approximately fifteen million prematurely delivered babies each year (3). In the Netherlands, seven to eight percent of all babies are born prematurely (1). Worldwide, around a million newborns die as a result of premature birth $(2,4)$. Survivors of preterm birth have higher rates of adverse long-term health outcomes including cerebral palsy, sensory deficits, learning disabilities, altered intestinal motility and respiratory illnesses compared with children born at term (5). The morbidity associated with preterm birth often extends to later life and has a severe impact on emotional and financial status of patients, their families, caregivers and society (6).

Preterm delivery can be classified into two main categories. The first category is classified as indicated preterm birth and consists of induced preterm labor or infants delivered by caesarean section, based on medical indications of maternal or fetal health (7). These include e.g. severe intra-uterine growth retardation (IUGR), maternal pre-eclampsia and HELLP syndrome (Hemolysis, Elevated Liver enzymes and Low Platelets) (8). The second category is classified as spontaneous preterm birth and consists of preterm labor with intact membranes and preterm premature rupture of the membranes (PPROM) $(7,9)$. In the latter, the risk of contamination and infection of the child is increased (1). Intra-uterine infection is the most common cause of spontaneous premature delivery $(10,11)$. As a result of an inflammatory reaction in and damage to the membranes, several enzymes, proteases and collagenases are released that initiate prostaglandin production (1). This can lead to premature dilation, contractions, PPROM, and ultimately premature birth.

\section{Chorioamnionitis}

Spontaneous preterm birth is in up to $40 \%$ associated with chorioamnionitis, defined as an inflammation of the chorion and amnion (10-13). Chorioamnionitis is inversely correlated to gestational age with the highest incidence in the most immature infants (14). Approximately $60 \%$ of preterm births below 25 weeks of gestation are associated with chorioamnionitis $(10,15)$, in contrast to $1-13 \%$ in term births (16-18).

Only a small proportion of pregnant women with preterm birth display clinical signs of chorioamnionitis such as maternal fever, uterine fundal tenderness, maternal tachycardia, fetal tachycardia, and purulent or foul amniotic fluid (19). However, preterm birth is most frequently the result of an asymptomatic chorioamnionitis. In these cases, pregnant women do not have clinical symptoms and the presence of chorioamnionitis is recognized after delivery. More precisely, histological examination after birth with proof of inflammation and necrosis throughout the chorionic plate and amnion is currently the gold standard to diagnose chorioamnionitis $(20,21)$. Consequently, the duration of fetal exposure to an inflammatory stimulus is seldom known. 
Several risk factors have been reported for chorioamnionitis, such as prolonged membrane rupture (including PPROM), prolonged labor, multiple digital vaginal exams with membrane rupture, nulliparity, group B streptococcus colonization, bacterial vaginosis, alcohol and tobacco use, meconium-stained amniotic fluid, internal monitoring and epidural anesthesia (12). Although PPROM is a major risk factor for clinical chorioamnionitis, it is noteworthy that together with preterm labor, PPROM frequently is the consequence of sub-clinical chorioamnionitis (22).

Chorioamnionitis typically results from a retrograde or ascending bacterial infection from the lower genital tract (cervix and vagina), resulting in microbial colonization of the chorioamnion and/or umbilical cord (figure 1) $(22,23)$. Other less common routes are: hematogenous/transplacental passage, iatrogenic infection complicating amniocentesis or chorionic villous sampling and anterograde infection from the peritoneum via the fallopian tubes (figure 1) (23).

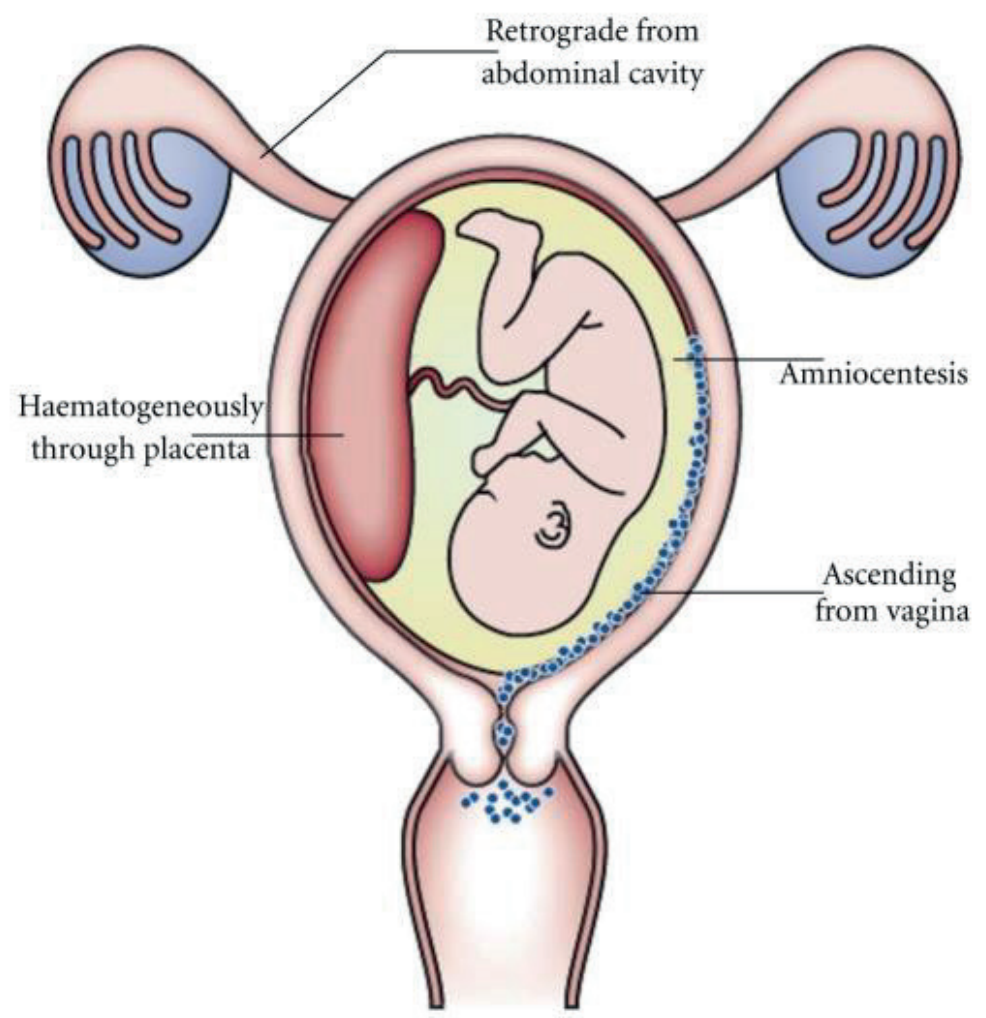

Figure 1. Potential routes of intra-uterine infection (7).

Various pathogens are identified in causing chorioamnionitis: E. coli, Fusobacterium, Streptococcus, Bacteriodes, Gardnerella species (spp.), Ureaplasma spp. (Ureaplasma 
urealyticum and Ureaplasma Parvum) and Mycoplasma spp. (Mycoplasma hominis), with the latter two being the most commonly identified (24-29). Chorioamnionitis is often polymicrobial, since over $65 \%$ of positive amniotic fluid cultures lead to the identification of two or more pathogens (12).

Chorioamnionitis can occur with intact membranes, which is especially common for the very small fastidious genital mycoplasmas such as Ureaplasma spp. and Mycoplasma hominis, found in the lower genital tract of over $70 \%$ of women (30). Hematogenous dissemination through the placenta is very rare, which can occur with a maternal Listeria monocytogenes infection (31).

The pathophysiology of a retrograde or ascending microbial invasion of the uterine cavity starts with invasion of the decidua, chorion and amnion with microbes from the lower genital tract. Subsequently, the microbes gain access to the amniotic cavity and infect the amniotic fluid. The presence of infectious microbes in the chorioamnion causes a maternal inflammatory response characterized by the release of IL-1, IL-6, IL-8, TNF- $a$ and matrix metalloproteinase (MMP) (12). The maternal inflammatory response may produce clinical chorioamnionitis and/or lead to prostaglandin release, cervical changes and weakened or ruptured membranes, with subsequent labor at term or premature birth (12).

As the fetus makes breathing movements and swallows the amniotic fluid, the lungs and intestine become directly exposed to bacterial components and inflammatory cytokines present in the amniotic fluid (32). This can induce an immune response in the fetus which is referred as the fetal inflammatory response syndrome (FIRS), characterized by increased systemic IL-6 and IL-8 levels in the fetus (33). The inflammatory response to chorioamnionitis is supposed to be the primary cause of injury to fetal organs (32). The combination of prematurity, FIRS and direct exposure to the infected amniotic fluid can induce multi-organ injury and may have long-term negative effects for the preterm infant (12).

Optimal management of clinical chorioamnionitis includes immediate initiation of broadspectrum antibiotic therapy and delivery to prevent both maternal and fetal complications $(12,31)$. Several studies have shown that the frequency of neonatal sepsis is reduced by up to $80 \%$ with intrapartum antibiotic treatment $(34,35)$. However, antibiotic treatment for clinical chorioamnionitis is only partially a solution.

\section{Necrotizing enterocolitis}

One of the most severe, life-threatening postnatal adverse effects of chorioamnionitis is necrotizing enterocolitis (NEC). NEC is the most common severe neonatal gastrointestinal (Gl) emergency that predominantly affects premature infants. It is an inflammatory intestinal disorder, characterized by variable damage to the intestinal tract, ranging from mucosal injury to full-thickness necrosis and perforation (36-40). The natural history of NEC is a fulminant progression with severe inflammation of the bowel, ischemia and necrosis, leading to septic shock, multiorgan failure, and death within hours or days of onset if appropriate treatment is not timely instituted. 
The risk of developing NEC is higher in neonates with a low birth weight. More than $85 \%$ of all NEC cases occur in very low birth weight premature infants $(<1500 \mathrm{~g},<32$ weeks of gestation), while only around $10 \%$ of cases occur in full-term newborns $(41,42)$. NEC incidences are still rising, because of increased early survival. Other known risk factors besides prematurity and low birth weight include formula feeding, mechanical ventilation, FIRS and chorioamnionitis $(19,41,43,44)$.

Mortality generally ranges from 15 to $30 \%$, but increases up to $50 \%$ for extremely low birth weight children treated surgically (45-47). Around $10 \%$ of Neonatal Intensive Care Unit (NICU) deaths can be attributed to NEC (48). Infants that do survive are at increased risk for e.g. growth retardation $(49,50)$, neurodevelopmental delay $(51,52)$, short bowel syndrome (53) and intestinal failure (54).

The timing of the onset of NEC is inversely correlated with the gestational age at delivery; in the severely premature neonate, onset often occurs in the fourth week after birth. In neonates born closer to term, onset typically occurs in the first week after birth (42).

The pathogenesis of NEC is multifactorial and still incompletely understood, however, immaturity of the intestinal immune system, abnormal bacterial colonization and immature gut motility are clearly involved in the etiology of the excessive inflammation $(41,46)$. As chorioamnionitis alters the amniotic environment, the fetal gut becomes predisposed to develop NEC either by direct bacterial colonization of the fetal intestine or by the immunological and structural changes that occur in the developing fetal gut following intra-uterine inflammation (55).

Neonates can present different symptoms varying from mild/moderate symptoms such as feeding intolerance, vomiting and abdominal distension to severe symptoms such as sepsis, metabolic shock, apnea and respiratory distress (55).

Despite advancing medical care, NEC treatment remains largely symptomatic. Indications for surgical intervention are pneumoperitoneum caused by bowel perforation and relentless systemic deterioration despite conservative medical management (41). However, effective preventative and therapeutic strategies are lacking (41).

An interesting category as a promising preventative intervention in NEC, might be pharmaceutical nutritional options, since NEC onset is closely related to the initiation of enteral feeding and enteral feeding is considered an important modifiable factor influencing NEC pathogenesis (56-58). Also the kind of enteral feeding is of importance, so is formula feeding associated with a risk for the development of NEC, while breast feeding reduces the risk of NEC (43). Plant sterols, also called phytosterols, are dietary constituents present in vegetable oils, nuts, grains and fruit (59) that are increasingly valued for their immune regulatory properties (60). Plant sterols are found in amniotic fluid $(61,62)$ and human breast milk (63-66), both fluids that protect against NEC $(67,68)$. Studies in rodent inflammatory bowel disease models and in human volunteers showed that plant sterols possess anti-inflammatory properties (69-71). Furthermore, recent literature showed in an ovine chorioamnionitis model that intra-amniotic plant sterol administration reduces fetal 
systemic inflammation and potentially gut inflammation (72), both factors predisposing to NEC development $(19,44)$. Therefore, enteral plant sterol administration during enteral feeding initiation might reduce intestinal inflammation and therewith might lead to a reduction of NEC incidence and severity. An option next to enrichment of formula feeding with plant sterols, might be increasing maternal plant sterol intake during the lactating period to therewith increase the plant sterol concentrations in breast milk.

\section{Enteric nervous system}

The enteric nervous system (ENS) is the largest and most complex part of the peripheral nervous system (PNS). The ENS is located in the walls of the entire gastro-intestinal (GI) tract and associated glands (salivary glands and pancreas) as well as the gallbladder. In humans, the ENS contains about 200-600 million neurons. The ENS of the tubular digestive tract (esophagus, stomach and intestines) is organized into an interconnected network (plexuses) of neurons and supportive cells (glial cells) that are grouped into ganglia located in two major plexuses: the submucosal or Meissner's plexus and the myenteric or Auerbach's plexus (figure 2) (73). Submucosal plexuses are solely located in the submucosa of the small and large intestine. The myenteric plexuses are located between the inner and outer layers of the muscularis externa extending from the upper esophagus to the internal anal sphincter (74).

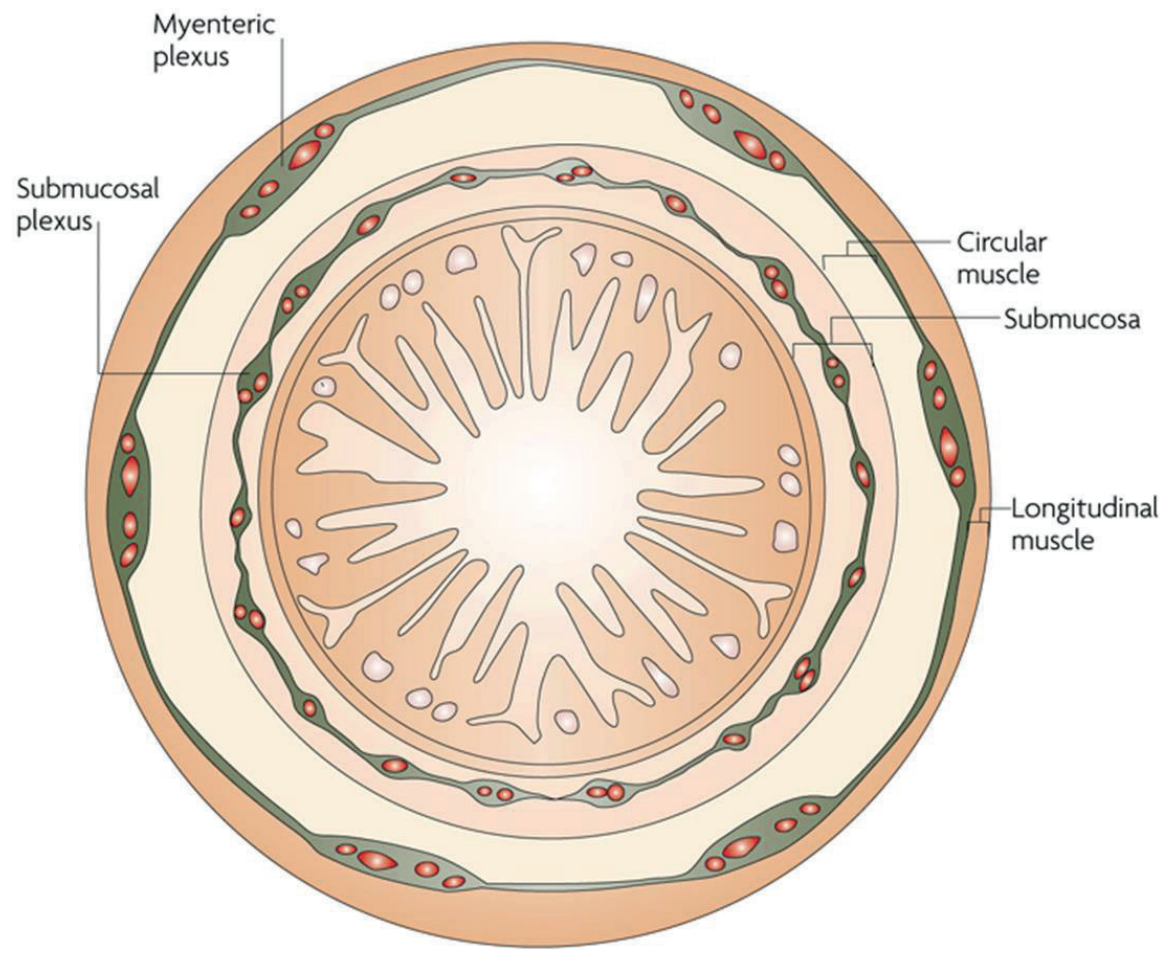

Figure 2. Transversal section of the gut. The neurons and glial cells of the ENS are grouped into two major plexuses: the submucosal plexus and the myenteric plexus (75). 
The submucosal and myenteric plexuses have different functions, which are all autonomously regulated. The submucosal plexus is responsible for e.g. coordinating the exchange of fluids across the mucosal surface, secretion of hormones, absorption and local blood flow. The myenteric plexus is primarily responsible for coordinating contractions and relaxations of gut smooth muscle involved in gut motility (73).

The ENS receives input from the extrinsic parasympathetic (via the vagus nerve and pelvic nerves) and sympathetic (via prevertebral ganglia) nervous systems (figure 3) (73). The information flow between the ENS and central nervous system (CNS) and between the ENS and sympathetic prevertebral ganglia is bidirectional.

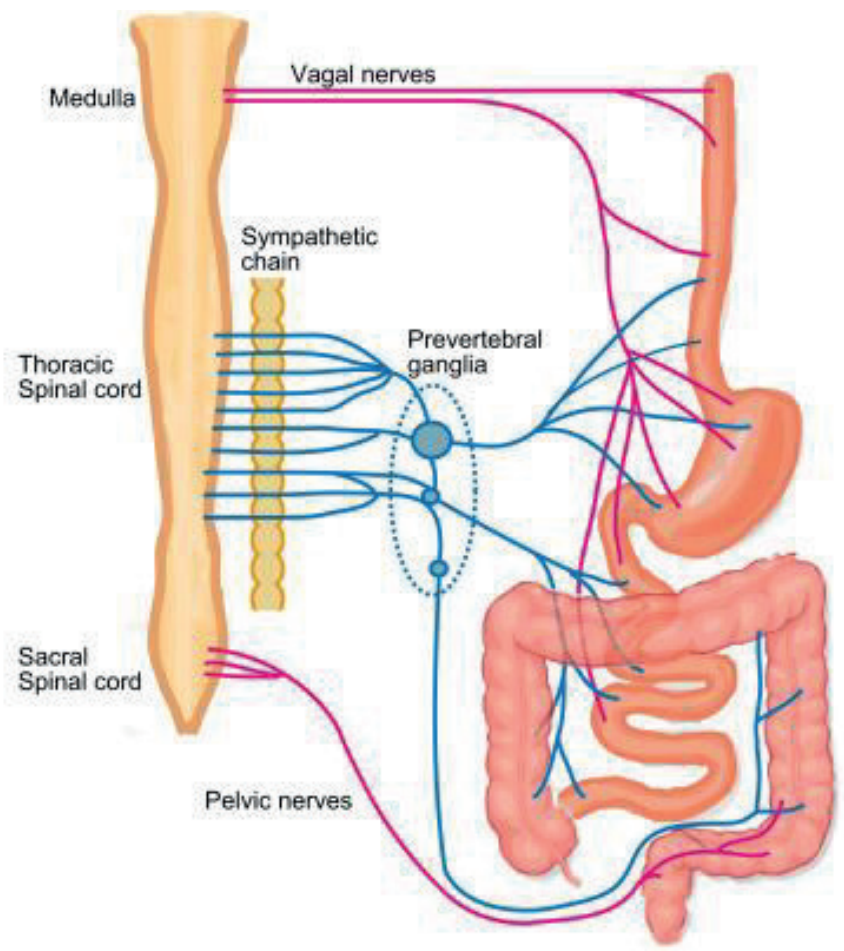

Figure 3. Extrinsic sympathetic (blue) and parasympathetic (pink) innervation of the Gl tract (76).

The ENS can act autonomously from any extrinsic innervation since enteric neurons are organized in microcircuits that contain intrinsic primary afferent neurons that can respond intrinsically to local stimuli to integrate information and coordinate motor output (73). The ENS is therefore unique in having both sensory and motor properties. The GI tract also harbors an extensive endocrine signaling system, and many Gl functions are under dual neuronal and endocrine control. In addition, the ENS interacts with the extensive intrinsic immune system of the Gl tract (73). 
Approximately 20 types of different enteric neurons can be defined based on their functions in the ENS. These various types can be classified in three groups: sensory neurons, interneurons and motor neurons (73). Sensory neurons or intrinsic primary afferent neurons (IPANs) can be subdivided in mucosal chemosensors, mucosal mechanoreceptors and stretch responsive neurons (77). They detect the physical state of the organs via chemical features of the luminal content and mechanical stimuli (e.g. stroking or tension in the gut wall). They react to these signals to initiate appropriate reflex control of motility, secretion and blood flow (77). IPANs connect with each other, with interneurons and directly with motor neurons.

Interneurons connect with other interneurons and with motor neurons to form chains that extend along the gut. At least one type of ascending and three types of descending interneurons have been identified, of which most of the interneurons are from the descending type (78). The ascending interneurons are mainly cholinergic, while the descending interneurons express a combination of different neurotransmitters including choline acetyltransferase (CHAT), neuronal nitric oxide synthase (nNOS), vasoactive intestinal polypeptide (VIP), serotonin $(5-H T)$, and somatostatin. nNOS, VIP and somatostatin neurons are involved in local motility reflexes, whereas 5-HT neurons are involved in the local secretomotor reflexes. Neurons using CHAT are involved in both reflexes $(77,78)$.

Motor neurons can be subdivided in muscle motor neurons, secretomotor neurons and secretomotor/vasodilator neurons (77). The muscle motor neurons innervate the longitudinal and circular muscles and the muscularis mucosae throughout the digestive tract and are therefore responsible for coordinating peristalsis. The muscle motor neurons are either excitatory (cholinergic and tachynergic) or inhibitory (e.g. nNOS) regulating muscle contraction or relaxation (77).

Secretomotor neurons regulate the exchange of water and electrolytes between the gut lumen and tissue fluid compartments by controlling the permeability of the mucosa to ions in the small and large intestines (77). Secretion is integrated with vasodilatation, which provides some of the fluid that is secreted. Further, vasodilator neurons regulate the local blood flow to the mucosa (77).

Enteric glial cells are known as the"supporting cells" of the nervous system. They outnumber enteric neurons by a factor of 4 to 10 (79) and surround the neuronal cell bodies tightly to protect the enteric neurons from potentially harmful extra ganglionic substances (80). Enteric glia share many structural and functional similarities to astrocytes in the CNS. They establish the homeostatic environment within enteric ganglia and function to provide structural and nutritional support for enteric neurons (81). Furthermore, enteric glial cells are known to contribute to neuronal maintenance, survival, function and are capable of generating enteric neurons in response to injury (82-84).

Mucosal enteric glial cells also interact with nonneuronal cells such as enterocytes, immune cells and enteroendocrine cells that are important in the maintenance of Gl homeostasis. Glial cells influence the development and maturation of enterocytes, can cause formation 
of more tight junctions in the epithelium and reduce epithelial permeability, contributing to the maintenance or enhancement of the Gl epithelial barrier $(79,85-89)$.

Glial cells have immunomodulatory effects and activated glial cells play a substantial role in the progression and inhibition of inflammation in the ENS. They can secrete cytokines and chemokines, which plays an important role in the activation and recruitment of immune cells (81). Moreover, enteric glial cells have phagocytic capabilities and can present antigens to innate and adaptive immune cells (81). On the other hand, enteric glial cells are capable of immunosuppression by the release of anti-inflammatory interleukins and by suppressing lymphocyte proliferation $(90,91)$.

Less is known about the interaction between mucosal glial cells and enteroendocrine cell, but specialized connections between the two cell types suggest that glia may have the potential to influence Gl hormone release (92). The fundamental roles of barrier regulation and hormone release in Gl physiology suggest that defective interactions between enteric glia and enterocytes or enteroendocrine cells could contribute to Gl inflammation (92).

The ENS originates from neural crest cells that colonize the gut during intra-uterine life. Formation of the ENS requires a complex coordinated migration, proliferation and differentiation, directed neurite growth, and establishment of a network of interconnected neurons $(93,94)$. Consequently, defects in ENS development can lead to several GI diseases (94). The ENS becomes functional in the last third of gestation in human, and continues to develop following birth $(73,93)$.

Functional and structural ENS abnormalities of both the submucosal and myenteric plexus have been found in rodent NEC models and neonates with NEC. Both in rodent NEC models and in specimen of neonates with NEC, a loss of enteric neurons and glial cells was observed in the submucosal and myenteric plexus (95-99). This loss of neurons might be explained by increased ENS apoptosis (96). Specifically, a reduced amount of nNOS-positive cells or decreased nNOS expression was found in the myenteric plexus (96, $97,100,101)$. In specimen of neonates with NEC, also the mRNA expression of CHAT in the myenteric plexus was decreased (96). These alterations suggests that ENS functions are disrupted. In a rodent NEC model, the suggested disrupted ENS functions were objectified by measuring methylene blue dye migration, showing that pups exposed to the NEC protocol had significantly decreased intestinal motility compared with control pups (95).

The number of nNOS-expressing neurons and glial cells remained low in the intestines from NEC patients even months after the acute episode when the patients underwent stoma closure (96). This decreased $\mathrm{nNOS}$ expression may explain the intestinal dysmotility seen in NEC patients even after recovery from the acute event (96).

We propose that the neuropathological lesions found in NEC have their origin far before the manifestation of NEC and that particularly the ENS alterations result in dysfunctional intestinal motility that facilitates intraluminal bacterial overgrowth and translocation, therewith promoting the multifactorial pathophysiology of NEC. We suggest that the involvement of chorioamnionitis in the induction of adverse intestinal outcomes including 
NEC combined with the presence of ENS abnormalities in NEC, makes it plausible that these alterations might already have their origin in utero due to the impact of an antenatal infection on the ENS.

In literature, several promising treatments have been proposed as treatments for the ENS alterations found in NEC, namely the hormone ghrelin, heparin-binding epidermal growth factor-like growth factor and neural stem cell transplantation $(95,96,102)$. These treatments were in one way or another able to recover the NEC-induced loss of enteric neurons and glial cells, promote enteric nerve regeneration by for instance promoting glial cell-derived neurogenesis and improve intestinal transit $(95,96,102)$.

\section{Enterohepatic circulation of bile acids}

Bile acids (BAs), the primary component of bile, have an important role in facilitating the digestion and absorption of lipids and lipid-soluble vitamins by micelle formation (103). Hepatocytes synthesize primary BAs from cholesterol through two different complex enzymatic pathways. The neutral or classical pathway is initiated by cholesterol 7a-hydroxylase (CYP7A1), which is the rate-limiting enzyme in the conversion of cholesterol to BAs (104). The acidic or alternative pathway is initiated by cytochrome P450 family 27 subfamily A member 1 (CYP27A1) and plays a more significant role in the BAs production in neonates (104). Cholic acid (CA) and chenodeoxycholic acid (CDCA) are the major primary BAs synthesized in the human liver, and are conjugated with taurine or glycine for secretion into the bile $(105,106)$. Conjugation of BAs is necessary to render them less toxic in the liver, to minimize passive absorption to achieve high luminal BAs concentrations in the intestine and to make them resistant to cleavage by pancreatic carboxypeptidases (104). The conjugated BAs are transported from the hepatocytes into the bile canaliculi via the bile salt export pump (BSEP) and released into the gallbladder and subsequently in the duodenum. Most conjugated BAs are reabsorbed in the brush border membrane of the terminal ileum through active uptake by the apical sodium-dependent bile acid transporter (ASBT) (107). Within the intestinal lumen, particularly in the colon, conjugated BAs which have failed to be absorbed via the ASBT are deconjugated and metabolized by the gut microbiota into secondary BAs. CA is formed into deoxycholic acid (DCA) and CDCA is formed into lithocholic acid (LCA), which are the major secondary BAs in humans (108). About $20-50 \%$ of formed DCA is reabsorbed by passive diffusion in the colon, while most of LCA is lost in the feces (107). In total, about $95 \%$ of the BAs are reabsorbed into intestinal epithelial cells. The remaining $5 \%$ is lost in the feces and are compensated by de novo synthesis in the liver to maintain a constant BA pool (104).

Following uptake by ASBT into the enterocyte, BAs bind to the ileal bile acid-binding protein (IBABP) to traverse the cytoplasm of the epithelial cells. BAs exit the basolateral site of the enterocyte via the organic solute transporter $a$ and $\beta$ dimer (OSTa/OST $\beta$ ) (109). Once in the interstitium, it is unclear how BAs enter the villus capillaries to gain access to the portal circulation, but this likely occurs by a passive process (107). BAs are transported bound to albumin via the portal vein back to the liver. The sinusoidal $\mathrm{Na}^{+}$-taurocholate cotransporting polypeptide (NTCP) takes up the BAs into the hepatocyte to be recycled. BAs can be recycled 4-12 times per day between hepatocytes and enterocytes, which is called the enterohepatic circulation ([EHC]; figure 4) (104). 


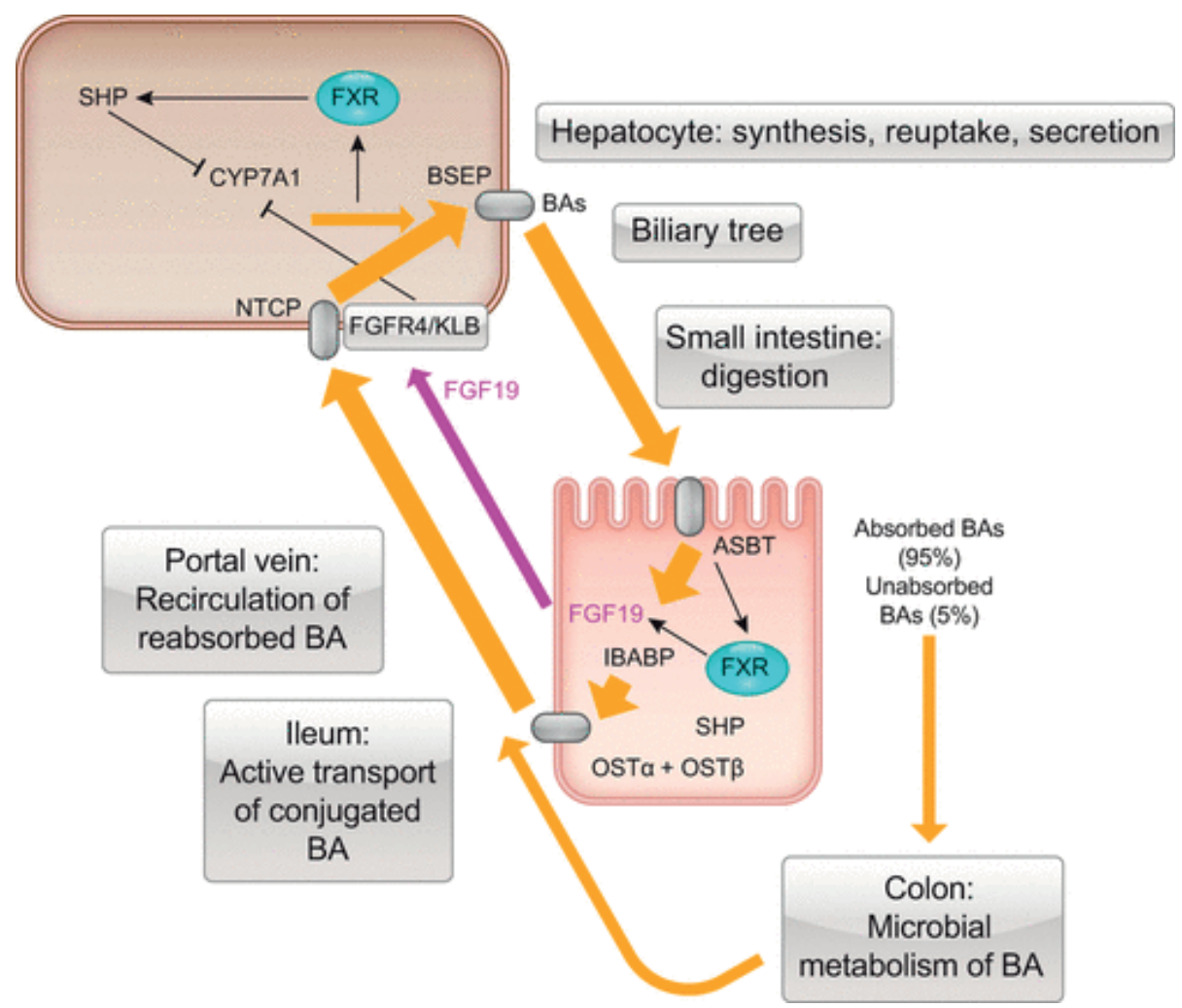

Figure 4. The enterohepatic circulation of bile acids (107).

Each step of the EHC, from hepatic synthesis, to intestinal reabsorption, and reuptake into hepatocytes, is regulated by complex pathways with cellular and molecular communication along the gut-liver axis. BAs themselves play a major role in activating these pathways, primarily by binding on the farnesoid $X$ receptor ( $F X R$ ) expressed in hepatocytes and epithelial cells lining the intestinal lumen (107). FXR regulates biliary BA secretion, intestinal BA absorption and secretion, inter-enterocyte transport and hepatic uptake of BAs (104). In addition, ileal FXR regulates BAs synthesis upon BAs binding, stimulating fibroblast growth factor 19 (FGF19) transcription. FGF19 inhibits BAs synthesis by inhibiting CYP7A1 and CYP27A1 (figure 4) (110).

Disruptions to the processes that control the synthesis, recycling, and excretion of BAs are associated with the onset of many diseases that can affect the intestine and adjacent organs (107). Alterations in the EHC have been found to contribute to the pathophysiology of NEC. Several studies have found that elevated ileal BAs and an altered expression of several BAs transporters may be a mechanism by which ileal damage occurs (111-115). It was shown that intraluminal accumulation of BAs resulted in intestinal epithelial damage similar to the histopathological findings in NEC (111). These high concentrations of intraluminal BAs might be the result of increased BA synthesis, which was found in a rat NEC model (112). 
High concentrations of intraluminal BAs resulted in their accumulation within enterocytes, with concomitant enterocyte damage (113). Preterm infants and rodents with NEC were found to have an increased expression of ASBT, suggesting increased BAs uptake into the enterocyte $(115,115)$. In addition, an insufficient transport from the apical to basolateral side of the ileum was suggested since rats with NEC had a decreased expression of IBABP (114). Given the role of BAs in NEC pathogenesis and the fact that fecal BA levels are higher in preterm infants in the week preceding NEC manifestation compared with control (113), one could speculate that EHC alterations might already have their origin in utero.

\section{Gut-liver axis}

The intestinal tract and the liver are anatomically and physiologically connected. The relationship between the two organs has been called the gut-liver axis. A special association between the liver and gut is also underlined by the common developmental origin of hepatocytes and intestinal epithelium from the ventral foregut endoderm (116).

The intestinal tract and the liver are anatomically connected through the portal vein, which drains blood from the mesenteric veins of the intestinal tract. Consequently, the liver is the primary recipient of gut-derived products, most prominently dietary nutrients and microbial components. Many dietary nutrients are taken up by hepatocytes and then metabolized. Moreover, the liver protects the body by clearing microbial products, toxins and microorganisms (such as bacteria and fungi) that have crossed the intestinal barrier (117). The cells responsible for the latter are the Kupffer cells, which are strategically located to clear the portal circulation of pathogens from the gut. If the intestinal barrier is disrupted, microbial exposure of the liver is enhanced and pathologic effects in the liver can be induced such as liver inflammation, hepatocyte death and parenchyma destruction (108). Bacteria and their products can be detrimental in the liver, but on the other hand have the beneficial effect of promoting liver regeneration (117).

The intestinal tract and the liver are physiologically connected through the EHC of BAs and the production of $\lg A$ antibodies. The liver influences the intestinal microbiota and helps to defend the intestine against microbial pathogens via the production and secretion of BAs and IgA. BAs can exert direct effects on intestinal bacteria by causing membrane damage and disrupting protein and DNA functions, particularly in Gram-positive bacteria (117). In addition, BAs are recently found to be critical regulators of intestinal epithelial function (107). They have for example a role in the regulation of fluid secretion, epithelial cell proliferation, survival and apoptosis, tight junction function and permeability, mucus production and cytokine production (107).

IgA production by the liver, which is secreted into the gut via the bile, is important for controlling intestinal microbial loads as lack of lgA leads to a significant increase in the biomass of anaerobic microbes in the small intestine (118). Furthermore, IgA plays an important role in preventing invasion of intestinal pathogens by preventing the uptake of enteric antigens and the binding of enterotoxins and micro-organisms to the intestinal microvilli (119). 


\section{Sheep as a translational model of chorioamnionitis}

In the past years, large animal models have proven their utility in translational research. In line, large animal models and sheep in particular have been successfully used to understand and unravel the pathological mechanisms underlying chorioamnionitis and the effect of various intra-uterine triggers on different organs (120). There are several reasons for the successful use of ovine models in translational perinatal medicine: the development of and the developmental processes that occur in the ovine intestine, lungs and brain during gestation are highly similar to that of humans and therefore the use of fetal sheep as an animal model is widely accepted (figure 5) (121). For example, human and ovine intestinal villus and crypt formation occurs during gestation, whereas in rodents these intestinal structures develop near term gestation or postnatally (121). In addition, the relatively long gestational period in sheep (term at $\sim 150$ days) makes investigation of fetal pathologies at selected gestational/maturational stages and for longer periods of time feasible.
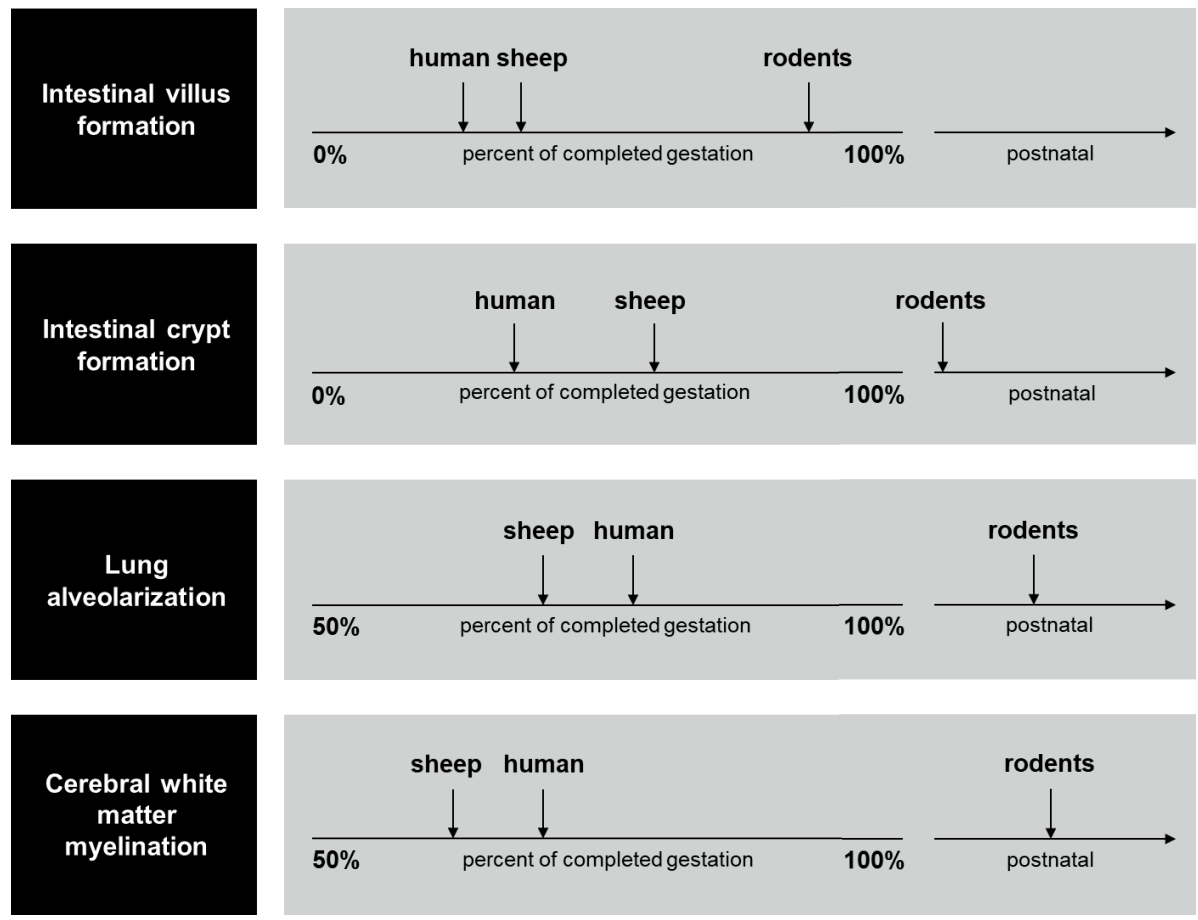

Figure 5. Differences through gestation in humans, sheep and rodents of various hallmarks of development of the fetal gut, lung and brain (121). 


\section{Outline of this thesis}

The aim of this thesis is to investigate the effect of an antenatal intra-uterine infection on the ENS and EHC of the fetus in order to gain essential insight in the relation between chorioamnionitis and the predisposition to adverse effects in fetal gut and liver outcomes. Moreover, we aim to investigate the ENS and intestinal motility postnatally to study the consequences of perinatal stress after birth. The aims and outcomes are described in several chapters.

Chorioamnionitis can occur with intact membranes, which is common for genital mycoplasmas, such as Ureaplasma spp., present in the lower genital tract of women (10, 122). The Ureaplasma spp. can cause chronic chorioamnionitis that does not evoke a maternal response, but is still associated with adverse fetal outcomes (123). In line, several studies have reported that Ureaplasma colonization in preterm infants is associated with NEC $(124,125)$. Therefore, in chapter $\mathbf{2}$, we explore the effects of acute LPS exposure and chronic IA UP on mucosa, submucosa and ENS of the terminal ileum in ovine fetuses. Since chorioamnionitis is often polymicrobial, with identification of two or more pathogens in over $65 \%$ of positive amniotic fluid cultures (12), we also investigate the effects of repetitive IA microbial exposure on the ovine fetal ileal mucosa, submucosa and ENS in chapter 2.

In chapter $\mathbf{3}$ we explore the time point specific effects (from 15 days to 5 hours before premature delivery) of intra-amniotic (IA) lipopolysaccharide (LPS) exposure on the ENS of the terminal ileum as inflammation is a dynamic process and the vulnerability of the fetus to injurious exposure during intra-uterine development varies. Understanding timedependent effects of intra-uterine inflammation is clinically important, as chorioamnionitis is often clinically silent and infants born after chorioamnionitis have been exposed to varying durations of intra-uterine inflammation when diagnosed.

Chapter $\mathbf{4}$ evaluates the ENS and intestinal motility postnatally in a NEC mouse model to investigate the consequences of perinatal stress after birth.

In chapter 5 the time-dependent effects of IA LPS administration from 15 days to 5 hours before premature delivery on the liver and EHC of premature sheep are being investigated, as inflammation is a dynamic process and the vulnerability of the fetus to injurious hits during the complex intra-uterine development varies.

In a recent study, chorioamnionitis induced by six days of intra-uterine UP exposure caused a reduced amount of conjugated BAs in the enterocytes of the terminal ileum of fetal sheep (72), which prompted us to investigate whether UP can cause changes in BAs related liver functions and the EHC. Therefore, in chapter $\mathbf{6}$, we investigate the effects of IA UP exposure on the liver and EHC in in a model of UP-induced chorioamnionitis in ovine fetuses. Furthermore, the prophylactic effects of IA plant sterols administration on the liver and EHC are being investigated as plant sterols have been shown to be anti-inflammatory in the liver of mice that developed non-alcoholic fatty liver disease (NASH) (126).

The main findings from the studies described in this thesis are integrated and discussed in a broader perspective in chapter $\mathbf{7}$. Clinical perspectives and potential implications for future research are also provided in this chapter. 


\section{References}

1. NVK/NVOG. Richtlijn Perinataal beleid bij extreme vroeggeboorte. Utrecht: Nederlandse Vereniging voor Obstetrie en Gynaecologie (NVOG). 2010.

2. Blencowe $\mathrm{H}$, Cousens S. Addressing the challenge of neonatal mortality. Trop Med Int Health. 2013;18(3):303-12 DOI: 10.1111/tmi.12048.

3. Howson CP, Kinney MV, McDougall L, Lawn JE, Born Too Soon Preterm Birth Action G. Born too soon: preterm birth matters. Reprod Health. 2013;10 Suppl 1:S1 DOI: 10.1186/1742-4755-10-S1S1.

4. Blencowe H, Cousens S, Oestergaard MZ, Chou D, Moller AB, Narwal R, Adler A, Vera Garcia C, Rohde S, Say L, Lawn JE. National, regional, and worldwide estimates of preterm birth rates in the year 2010 with time trends since 1990 for selected countries: a systematic analysis and implications. Lancet. 2012;379(9832):2162-72 DOI: 10.1016/S0140-6736(12)60820-4.

5. Beck S, Wojdyla D, Say L, Betran AP, Merialdi M, Requejo JH, Rubens C, Menon R, Van Look PF. The worldwide incidence of preterm birth: a systematic review of maternal mortality and morbidity. Bull World Health Organ. 2010;88(1):31-8 DOI: 10.2471/BLT.08.062554.

6. Hodek JM, von der Schulenburg JM, Mittendorf T. i. Health Econ Rev. 2011;1(1):6 DOI: 10.1186/2191-1991-1-6.

7. Goldenberg RL, Culhane JF, lams JD, Romero R. Epidemiology and causes of preterm birth. Lancet. 2008;371(9606):75-84 DOI: 10.1016/S0140-6736(08)60074-4.

8. Irminger-Finger I, Jastrow N, Irion O. Preeclampsia: a danger growing in disguise. Int J Biochem Cell Biol. 2008;40(10):1979-83 DOI: 10.1016/j.biocel.2008.04.006.

9. Menon R. Spontaneous preterm birth, a clinical dilemma: etiologic, pathophysiologic and genetic heterogeneities and racial disparity. Acta Obstet Gynecol Scand. 2008;87(6):590-600 DOI: 10.1080/00016340802005126.

10. Goldenberg RL, Hauth JC, Andrews WW. Intrauterine infection and preterm delivery. N Engl J Med. 2000;342(20):1500-7 DOI: 10.1056/NEJM200005183422007.

11. Lamont RF. Infection in the prediction and antibiotics in the prevention of spontaneous preterm labour and preterm birth. BJOG. 2003;110 Suppl 20:71-5.

12. Tita AT, Andrews WW. Diagnosis and management of clinical chorioamnionitis. Clin Perinatol. 2010;37(2):339-54 DOI: 10.1016/j.clp.2010.02.003.

13. Galinsky R, Polglase GR, Hooper SB, Black MJ, Moss TJ. The consequences of chorioamnionitis: preterm birth and effects on development. J Pregnancy. 2013;2013:412831 DOI: $10.1155 / 2013 / 412831$.

14. Lahra MM, Jeffery HE. A fetal response to chorioamnionitis is associated with early survival after preterm birth. Am J Obstet Gynecol. 2004;190(1):147-51 DOI: 10.1016/j.ajog.2003.07.012.

15. Romero R, Espinoza J, Chaiworapongsa T, Kalache K. Infection and prematurity and the role of preventive strategies. Semin Neonatol. 2002;7(4):259-74.

16. Alexander JM, McIntire DM, Leveno KJ. Chorioamnionitis and the prognosis for term infants. Obstet Gynecol. 1999;94(2):274-8 DOI: 10.1016/s0029-7844(99)00256-2.

17. Blume HK, Li Cl, Loch CM, Koepsell TD. Intrapartum fever and chorioamnionitis as risks for encephalopathy in term newborns: a case-control study. Dev Med Child Neurol. 2008;50(1):19-24 DOI: $10.1111 /$ j.1469-8749.2007.02007.x.

18. Seong HS, Lee SE, Kang JH, Romero R, Yoon BH. The frequency of microbial invasion of the amniotic cavity and histologic chorioamnionitis in women at term with intact membranes in the presence or absence of labor. Am J Obstet Gynecol. 2008;199(4):375 e1-5 DOI: 10.1016/j.ajog.2008.06.040. 
19. Gantert M, Been JV, Gavilanes AW, Garnier Y, Zimmermann LJ, Kramer BW. Chorioamnionitis: a multiorgan disease of the fetus? J Perinatol. 2010;30 Suppl:S21-30 DOI: 10.1038/jp.2010.96.

20. Redline RW. Inflammatory responses in the placenta and umbilical cord. Semin Fetal Neonatal Med. 2006;11(5):296-301 DOI: 10.1016/j.siny.2006.02.011.

21. Faye-Petersen OM. The placenta in preterm birth. J Clin Pathol. 2008;61(12):1261-75 DOI: 10.1136/ jcp.2008.055244.

22. Goldenberg RL, Andrews WW, Hauth JC. Choriodecidual infection and preterm birth. Nutr Rev. 2002;60(5 Pt 2):S19-25 DOI: 10.1301/00296640260130696.

23. Fahey JO. Clinical management of intra-amniotic infection and chorioamnionitis: a review of the literature. J Midwifery Womens Health. 2008;53(3):227-35 DOI: 10.1016/j.jmwh.2008.01.001.

24. Kwak DW, Hwang HS, Kwon JY, Park YW, Kim YH. Co-infection with vaginal Ureaplasma urealyticum and Mycoplasma hominis increases adverse pregnancy outcomes in patients with preterm labor or preterm premature rupture of membranes. J Matern Fetal Neonatal Med. 2014;27(4):333-7 DOI: 10.3109/14767058.2013.818124.

25. Martinez-Lopez DG, Funderburg NT, Cerissi A, Rifaie R, Aviles-Medina L, Llorens-Bonilla BJ, Sleasman J, Luciano AA. Lipopolysaccharide and soluble CD14 in cord blood plasma are associated with prematurity and chorioamnionitis. Pediatr Res. 2014;75(1-1):67-74 DOI: 10.1038/pr.2013.182.

26. Payne MS, Bayatibojakhi S. Exploring preterm birth as a polymicrobial disease: an overview of the uterine microbiome. Front Immunol. 2014;5:595 DOI: 10.3389/fimmu.2014.00595.

27. Romero R, Miranda J, Chaiworapongsa T, Chaemsaithong P, Gotsch F, Dong Z, Ahmed Al, Yoon $\mathrm{BH}$, Hassan SS, Kim CJ, Korzeniewski SJ, Yeo L. A novel molecular microbiologic technique for the rapid diagnosis of microbial invasion of the amniotic cavity and intra-amniotic infection in preterm labor with intact membranes. Am J Reprod Immunol. 2014;71(4):330-58 DOI: 10.1111/aji.12189.

28. Ericson JE, Laughon MM. Chorioamnionitis: implications for the neonate. Clin Perinatol. 2015;42(1):155-65, ix DOI: 10.1016/j.clp.2014.10.011.

29. Musilova I, Pliskova L, Kutova R, Hornychova H, Jacobsson B, Kacerovsky M. Ureaplasma species and Mycoplasma hominis in cervical fluid of pregnancies complicated by preterm prelabor rupture of membranes. J Matern Fetal Neonatal Med. 2016;29(1):1-7 DOI: 10.3109/14767058.2014.984606.

30. Eschenbach DA. Ureaplasma urealyticum and premature birth. Clin Infect Dis. 1993;17 Suppl 1:S100-6 DOI: 10.1093/clinids/17.supplement_1.s100.

31. Gibbs RS, Duff P. Progress in pathogenesis and management of clinical intraamniotic infection. Am J Obstet Gynecol. 1991;164(5 Pt 1):1317-26 DOI: 10.1016/0002-9378(91)90707-x.

32. Wolfs TG, Kramer BW, Thuijls G, Kemp MW, Saito M, Willems MG, Senthamarai-Kannan P, Newnham JP, Jobe AH, Kallapur SG. Chorioamnionitis-induced fetal gut injury is mediated by direct gut exposure of inflammatory mediators or by lung inflammation. Am J Physiol Gastrointest Liver Physiol. 2014;306(5):G382-93 DOI: 10.1152/ajpgi.00260.2013.

33. Gussenhoven R, Westerlaken RJJ, Ophelders D, Jobe AH, Kemp MW, Kallapur SG, Zimmermann LJ, Sangild PT, Pankratova S, Gressens P, Kramer BW, Fleiss B, Wolfs T. Chorioamnionitis, neuroinflammation, and injury: timing is key in the preterm ovine fetus. J Neuroinflammation. 2018;15(1):113 DOI: 10.1186/s12974-018-1149-x.

34. Sperling RS, Ramamurthy RS, Gibbs RS. A comparison of intrapartum versus immediate postpartum treatment of intra-amniotic infection. Obstet Gynecol. 1987;70(6):861-5.

35. Gilstrap LC, 3rd, Leveno KJ, Cox SM, Burris JS, Mashburn M, Rosenfeld CR. Intrapartum treatment of acute chorioamnionitis: impact on neonatal sepsis. Am J Obstet Gynecol. 1988;159(3):579-83 DOI: 10.1016/s0002-9378(88)80012-7. 
36. Berman L, Moss RL. Necrotizing enterocolitis: an update. Semin Fetal Neonatal Med. 2011;16(3):145-50 DOI: 10.1016/j.siny.2011.02.002.

37. Dominguez KM, Moss RL. Necrotizing enterocolitis. Clin Perinatol. 2012;39(2):387-401 DOI: 10.1016/j.clp.2012.04.011.

38. Choi YY. Necrotizing enterocolitis in newborns: update in pathophysiology and newly emerging therapeutic strategies. Korean J Pediatr. 2014;57(12):505-13 DOI: 10.3345/kjp.2014.57.12.505.

39. Neu J. Necrotizing enterocolitis: the mystery goes on. Neonatology. 2014;106(4):289-95 DOI: 10.1159/000365130.

40. Lim JC, Golden JM, Ford HR. Pathogenesis of neonatal necrotizing enterocolitis. Pediatr Surg Int. 2015;31(6):509-18 DOI: 10.1007/s00383-015-3697-9.

41. Neu J. Necrotizing enterocolitis. World Rev Nutr Diet. 2014;110:253-63 DOI: 10.1159/000358474.

42. Rich BS, Dolgin SE. Necrotizing Enterocolitis. Pediatr Rev. 2017;38(12):552-9 DOI: 10.1542/ pir.2017-0002.

43. Gephart SM, McGrath JM, Effken JA, Halpern MD. Necrotizing enterocolitis risk: state of the science. Adv Neonatal Care. 2012;12(2):77-87; quiz 8-9 DOI: 10.1097/ANC.0b013e31824cee94.

44. Been JV, Lievense S, Zimmermann LJ, Kramer BW, Wolfs TG. Chorioamnionitis as a risk factor for necrotizing enterocolitis: a systematic review and meta-analysis. J Pediatr. 2013;162(2):236-42 e2 DOI: 10.1016/j.jpeds.2012.07.012.

45. Blakely ML, Lally KP, McDonald S, Brown RL, Barnhart DC, Ricketts RR, Thompson WR, Scherer LR, Klein MD, Letton RW, Chwals WJ, Touloukian RJ, Kurkchubasche AG, Skinner MA, Moss $\mathrm{RL}$, Hilfiker ML, Network NECSotNNR. Postoperative outcomes of extremely low birth-weight infants with necrotizing enterocolitis or isolated intestinal perforation: a prospective cohort study by the NICHD Neonatal Research Network. Ann Surg. 2005;241(6):984-9; discussion 9-94 DOI: 10.1097/01.sla.0000164181.67862.7f.

46. Lin PW, Stoll BJ. Necrotising enterocolitis. Lancet. 2006;368(9543):1271-83 DOI: 10.1016/S01406736(06)69525-1.

47. Hull MA, Fisher JG, Gutierrez IM, Jones BA, Kang KH, Kenny M, Zurakowski D, Modi BP, Horbar JD, Jaksic T. Mortality and management of surgical necrotizing enterocolitis in very low birth weight neonates: a prospective cohort study. J Am Coll Surg. 2014;218(6):1148-55 DOI: 10.1016/j. jamcollsurg.2013.11.015.

48. Jacob J, Kamitsuka M, Clark RH, Kelleher AS, Spitzer AR. Etiologies of NICU deaths. Pediatrics. 2015;135(1):e59-65 DOI: 10.1542/peds.2014-2967.

49. Hintz SR, Kendrick DE, Stoll BJ, Vohr BR, Fanaroff AA, Donovan EF, Poole WK, Blakely ML, Wright L, Higgins R, Network NNR. Neurodevelopmental and growth outcomes of extremely low birth weight infants after necrotizing enterocolitis. Pediatrics. 2005;115(3):696-703 DOI: 10.1542/ peds.2004-0569.

50. Hong CR, Fullerton BS, Mercier CE, Morrow KA, Edwards EM, Ferrelli KR, Soll RF, Modi BP, Horbar JD, Jaksic T. Growth morbidity in extremely low birth weight survivors of necrotizing enterocolitis at discharge and two-year follow-up. J Pediatr Surg. 2018;53(6):1197-202 DOI: 10.1016/j. jpedsurg.2018.02.085.

51. Rees CM, Pierro A, Eaton S. Neurodevelopmental outcomes of neonates with medically and surgically treated necrotizing enterocolitis. Arch Dis Child Fetal Neonatal Ed. 2007;92(3):F193-8 DOI: 10.1136/adc.2006.099929.

52. Schulzke SM, Deshpande GC, Patole SK. Neurodevelopmental outcomes of very low-birth-weight infants with necrotizing enterocolitis: a systematic review of observational studies. Arch Pediatr Adolesc Med. 2007;161(6):583-90 DOI: 10.1001/archpedi.161.6.583. 
53. Mutanen A, Wales PW. Etiology and prognosis of pediatric short bowel syndrome. Semin Pediatr Surg. 2018;27(4):209-17 DOI: 10.1053/j.sempedsurg.2018.07.009.

54. Duggan CP, Jaksic T. Pediatric Intestinal Failure. N Engl J Med. 2017;377(7):666-75 DOI: 10.1056/ NEJMra1602650.

55. Coggins SA, Wynn JL, Weitkamp JH. Infectious causes of necrotizing enterocolitis. Clin Perinatol. 2015;42(1):133-54, ix DOI: 10.1016/j.clp.2014.10.012.

56. Caplan MS. Necrotizing Enterocolitis in Preterm Infants is Related to Enteral Feeding, But the Mechanisms Remain Uncertain and Have Changed Over Time. Current Pediatrics Reports. 2014;2(4):241-7 DOI: 10.1007/s40124-014-0062-8.

57. Christian VJ, Polzin E, Welak S. Nutrition Management of Necrotizing Enterocolitis. Nutr Clin Pract. 2018;33(4):476-82 DOI: 10.1002/ncp.10115.

58. Vaks Y, Birnie KL, Carmichael SL, Hernandez-Boussard T, Benitz WE. Temporal Relationship of Onset of Necrotizing Enterocolitis and Introduction of Enteric Feedings and Powdered Milk Fortifier. Am J Perinatol. 2018;35(7):616-23 DOI: 10.1055/s-0037-1608928.

59. Gylling H, Plat J, Turley S, Ginsberg HN, Ellegard L, Jessup W, Jones PJ, Lutjohann D, Maerz W, Masana L, Silbernagel G, Staels B, Boren J, Catapano AL, De Backer G, Deanfield J, Descamps OS, Kovanen PT, Riccardi G, Tokgozoglu L, Chapman MJ. Plant sterols and plant stanols in the management of dyslipidaemia and prevention of cardiovascular disease. Atherosclerosis. 2014;232(2):346-60 DOI: 10.1016/j.atherosclerosis.2013.11.043.

60. Brull F, De Smet E, Mensink RP, Vreugdenhil A, Kerksiek A, Lutjohann D, Wesseling G, Plat J. Dietary plant stanol ester consumption improves immune function in asthma patients: results of a randomized, double-blind clinical trial. Am J Clin Nutr. 2016;103(2):444-53 DOI: 10.3945/ ajcn.115.117531.

61. Amaral C, Gallardo E, Rodrigues R, Pinto Leite R, Quelhas D, Tomaz C, Cardoso ML. Quantitative analysis of five sterols in amniotic fluid by GC-MS: application to the diagnosis of cholesterol biosynthesis defects. J Chromatogr B Analyt Technol Biomed Life Sci. 2010;878(23):2130-6 DOI: 10.1016/j.jchromb.2010.06.010.

62. Baardman ME, Erwich JJ, Berger RM, Hofstra RM, Kerstjens-Frederikse WS, Lutjohann D, Plosch T. The origin of fetal sterols in second-trimester amniotic fluid: endogenous synthesis or maternal-fetal transport? Am J Obstet Gynecol. 2012;207(3):202 e19-25 DOI: 10.1016/j.ajog.2012.06.003.

63. Mellies MJ, Burton K, Larsen R, Fixler D, Glueck CJ. Cholesterol, phytosterols, and polyunsaturated/ saturated fatty acid ratios during the first 12 months of lactation. The American Journal of Clinical Nutrition. 1979;32(12):2383-9 DOI: 10.1093/ajcn/32.12.2383.

64. Laitinen K, Isolauri E, Kaipiainen L, Gylling H, Miettinen TA. Plant stanol ester spreads as components of a balanced diet for pregnant and breast-feeding women: evaluation of clinical safety. Br J Nutr. 2009;101(12):1797-804 DOI: 10.1017/S0007114508133608.

65. Benoit B, Fauquant C, Daira P, Peretti N, Guichardant M, Michalski M-C. Phospholipid species and minor sterols in French human milks. Food chemistry. 2010;120(3):684-91 DOI: 10.1016/j. foodchem.2009.10.061.

66. Hamdan IJA, Sanchez-Siles LM, Matencio E, Bermudez JD, Garcia-Llatas G, Lagarda MJ. Sterols in human milk during lactation: bioaccessibility and estimated intakes. Food Funct. 2018;9(12):656676 DOI: 10.1039/c8fo01795f.

67. Lucas A, Cole TJ. Breast milk and neonatal necrotising enterocolitis. Lancet. 1990;336(8730):151923.

68. Dasgupta S, Jain SK. Protective effects of amniotic fluid in the setting of necrotizing enterocolitis. Pediatr Res. 2017;82(4):584-95 DOI: 10.1038/pr.2017.144. 
69. Lee IA, Kim EJ, Kim DH. Inhibitory effect of beta-sitosterol on TNBS-induced colitis in mice. Planta Med. 2012;78(9):896-8 DOI: 10.1055/s-0031-1298486.

70. Aldini R, Micucci M, Cevenini M, Fato R, Bergamini C, Nanni C, Cont M, Camborata C, Spinozzi S, Montagnani M, Roda G, D'Errico-Grigioni A, Rosini F, Roda A, Mazzella G, Chiarini A, Budriesi R. Antiinflammatory effect of phytosterols in experimental murine colitis model: prevention, induction, remission study. PLoS One. 2014;9(9):e108112 DOI: 10.1371/journal.pone.0108112.

71. te Velde AA, Brull F, Heinsbroek SE, Meijer SL, Lutjohann D, Vreugdenhil A, Plat J. Effects of Dietary Plant Sterols and Stanol Esters with Low- and High-Fat Diets in Chronic and Acute Models for Experimental Colitis. Nutrients. 2015;7(10):8518-31 DOI: 10.3390/nu7105412.

72. van Gorp C, de Lange IH, Spiller OB, Dewez F, Cillero Pastor B, Heeren RMA, Kessels L, Kloosterboer N, van Gemert WG, Beeton ML, Stock SJ, Jobe AH, Payne MS, Kemp MW, Zimmermann LJ, Kramer BW, Plat J, Wolfs T. Protection of the Ovine Fetal Gut against Ureaplasma-Induced Chorioamnionitis: A Potential Role for Plant Sterols. Nutrients. 2019;11(5) DOI: 10.3390/nu11050968.

73. Furness J. The Enteric Nervous System. Oxford, UK: Blackwell. 2006.

74. Furness JB, Callaghan BP, Rivera LR, Cho HJ. The enteric nervous system and gastrointestinal innervation: integrated local and central control. Adv Exp Med Biol. 2014;817:39-71 DOI: 10.1007/978-1-4939-0897-4_3.

75. Heanue TA, Pachnis V. Enteric nervous system development and Hirschsprung's disease: advances in genetic and stem cell studies. Nat Rev Neurosci. 2007;8(6):466-79 DOI: 10.1038/nrn2137.

76. Uesaka T, Young HM, Pachnis V, Enomoto H. Development of the intrinsic and extrinsic innervation of the gut. Dev Biol. 2016;417(2):158-67 DOI: 10.1016/j.ydbio.2016.04.016.

77. Furness JB. Types of neurons in the enteric nervous system. J Auton Nerv Syst. 2000;81(1-3):87-96 DOI: 10.1016/s0165-1838(00)00127-2.

78. Hansen MB. The enteric nervous system I: organisation and classification. Pharmacol Toxicol. 2003;92(3):105-13 DOI: 10.1034/j.1600-0773.2003.t01-1-920301.x.

79. Neunlist M, Aubert P, Bonnaud S, Van Landeghem L, Coron E, Wedel T, Naveilhan P, Ruhl A, Lardeux B, Savidge T, Paris F, Galmiche JP. Enteric glia inhibit intestinal epithelial cell proliferation partly through a TGF-beta1-dependent pathway. Am J Physiol Gastrointest Liver Physiol. 2007;292(1):G231-41 DOI: 10.1152/ajpgi.00276.2005.

80. Savidge TC, Sofroniew MV, Neunlist M. Starring roles for astroglia in barrier pathologies of gut and brain. Lab Invest. 2007;87(8):731-6 DOI: 10.1038/labinvest.3700600.

81. Chow AK, Gulbransen BD. Potential roles of enteric glia in bridging neuroimmune communication in the gut. Am J Physiol Gastrointest Liver Physiol. 2017;312(2):G145-G52 DOI: 10.1152/ ajpgi.00384.2016.

82. Joseph NM, He S, Quintana E, Kim YG, Nunez G, Morrison SJ. Enteric glia are multipotent in culture but primarily form glia in the adult rodent gut. J Clin Invest. 2011;121(9):3398-411 DOI: 10.1172/ JCl58186.

83. Laranjeira C, Sandgren K, Kessaris N, Richardson W, Potocnik A, Vanden Berghe P, Pachnis V. Glial cells in the mouse enteric nervous system can undergo neurogenesis in response to injury. J Clin Invest. 2011;121(9):3412-24 DOI: 10.1172/jci58200.

84. De Giorgio R, Giancola F, Boschetti E, Abdo H, Lardeux B, Neunlist M. Enteric glia and neuroprotection: basic and clinical aspects. Am J Physiol Gastrointest Liver Physiol. 2012;303(8):G887-93 DOI: 10.1152/ajpgi.00096.2012.

85. Savidge TC, Newman P, Pothoulakis C, Ruhl A, Neunlist M, Bourreille A, Hurst R, Sofroniew MV. Enteric glia regulate intestinal barrier function and inflammation via release of S-nitrosog/utathione. Gastroenterology. 2007;132(4):1344-58 DOI: 10.1053/j.gastro.2007.01.051. 
86. Cheadle GA, Costantini TW, Lopez N, Bansal V, Eliceiri BP, Coimbra R. Enteric glia cells attenuate cytomix-induced intestinal epithelial barrier breakdown. PLoS One. 2013;8(7):e69042 DOI: 10.1371/journal.pone.0069042.

87. Xiao W, Wang W, Chen W, Sun L, Li X, Zhang C, Yang H. GDNF is involved in the barrier-inducing effect of enteric glial cells on intestinal epithelial cells under acute ischemia reperfusion stimulation. Mol Neurobiol. 2014;50(2):274-89 DOI: 10.1007/s12035-014-8730-9.

88. Meir M, Flemming S, Burkard N, Bergauer L, Metzger M, Germer CT, Schlegel N. Glial cell linederived neurotrophic factor promotes barrier maturation and wound healing in intestinal epithelial cells in vitro. Am J Physiol Gastrointest Liver Physiol. 2015;309(8):G613-24 DOI: 10.1152/ ajpgi.00357.2014.

89. Li Z, Zhang X, Zhou H, Liu W, Li J. Exogenous S-nitrosoglutathione attenuates inflammatory response and intestinal epithelial barrier injury in endotoxemic rats. J Trauma Acute Care Surg. 2016;80(6):977-84 DOI: 10.1097/TA.0000000000001008.

90. Ibiza S, Garcia-Cassani B, Ribeiro H, Carvalho T, Almeida L, Marques R, Misic AM, BartowMcKenney C, Larson DM, Pavan WJ, Eberl G, Grice EA, Veiga-Fernandes H. Glial-cell-derived neuroregulators control type 3 innate lymphoid cells and gut defence. Nature. 2016;535(7612):4403 DOI: $10.1038 /$ nature 18644.

91. Kermarrec L, Durand T, Neunlist M, Naveilhan P, Neveu I. Enteric glial cells have specific immunosuppressive properties. J Neuroimmunol. 2016;295-296:79-83 DOI: 10.1016/j. jneuroim.2016.04.011.

92. Bohorquez DV, Samsa LA, Roholt A, Medicetty S, Chandra R, Liddle RA. An enteroendocrine cellenteric glia connection revealed by 3D electron microscopy. PLoS One. 2014;9(2):e89881 DOI: 10.1371/journal.pone.0089881.

93. Burns AJ, Roberts RR, Bornstein JC, Young HM. Development of the enteric nervous system and its role in intestinal motility during fetal and early postnatal stages. Semin Pediatr Surg. 2009;18(4):196-205 DOI: 10.1053/j.sempedsurg.2009.07.001.

94. Lake J, Heuckeroth RO. Entericnervous system development:migration, differentiation, and disease. Am J Physiol Gastrointest Liver Physiol. 2013;305(1):G1-24 DOI: 10.1152/ajpgi.00452.2012.

95. Wei J, Zhou Y, Besner GE. Heparin-binding EGF-like growth factor and enteric neural stem cell transplantation in the prevention of experimental necrotizing enterocolitis in mice. Pediatr Res. 2015;78(1):29-37 DOI: 10.1038/pr.2015.63.

96. Zhou Y, Yang J, Watkins DJ, Boomer LA, Matthews MA, Su Y, Besner GE. Enteric nervous system abnormalities are present in human necrotizing enterocolitis: potential neurotransplantation therapy. Stem Cell Res Ther. 2013;4(6):157 DOI: 10.1186/scrt387.

97. Sigge W, Wedel T, Kuhnel W, Krammer HJ. Morphologic alterations of the enteric nervous system and deficiency of non-adrenergic non-cholinergic inhibitory innervation in neonatal necrotizing enterocolitis. Eur J Pediatr Surg. 1998;8(2):87-94 DOI: 10.1055/s-2008-1071128.

98. Wedel T, Krammer HJ, Kuhnel W, Sigge W. Alterations of the enteric nervous system in neonatal necrotizing enterocolitis revealed by whole-mount immunohistochemistry. Pediatr Pathol Lab Med. 1998;18(1):57-70.

99. Fagbemi AO, Torrente F, Puleston J, Lakhoo K, James S, Murch SH. Enteric neural disruption in necrotizing enterocolitis occurs in association with myenteric glial cell CCL20 expression. J Pediatr Gastroenterol Nutr. 2013;57(6):788-93 DOI: 10.1097/MPG.0b013e3182a86fd4.

100. Meister AL, Doheny KK, Travagli RA. Necrotizing enterocolitis attenuates developmental heart rate variability increases in newborn rats. Neurogastroenterol Motil. 2019;31(3):e13484 DOI: 10.1111/ nmo.13484. 
101.Zhou Y, Wang Y, Olson J, Yang J, Besner GE. Heparin-binding EGF-like growth factor promotes neuronal nitric oxide synthase expression and protects the enteric nervous system after necrotizing enterocolitis. Pediatr Res. 2017;82(3):490-500 DOI: 10.1038/pr.2017.68.

102. Meister AL, Burkholder CR, Doheny KK, Travagli RA. Ghrelin ameliorates the phenotype of newborn rats induced with mild necrotizing enterocolitis. Neurogastroenterol Motil. 2019;31(11):e13682 DOI: $10.1111 / \mathrm{nmo} .13682$.

103. Russell DW. The enzymes, regulation, and genetics of bile acid synthesis. Annu Rev Biochem. 2003;72:137-74 DOI: 10.1146/annurev.biochem.72.121801.161712.

104. Chiang JY. Bile acid metabolism and signaling. Compr Physiol. 2013;3(3):1191-212 DOI: 10.1002/ cphy.c120023.

105. Hylemon PB, Zhou H, Pandak WM, Ren S, Gil G, Dent P. Bile acids as regulatory molecules. J Lipid Res. 2009;50(8):1509-20 DOI: 10.1194/jlr.R900007-JLR200.

106. Zhou H, Hylemon PB. Bile acids are nutrient signaling hormones. Steroids. 2014;86:62-8 DOI: 10.1016/j.steroids.2014.04.016.

107. Hegyi P, Maleth J, Walters JR, Hofmann AF, Keely SJ. Guts and Gall: Bile Acids in Regulation of Intestinal Epithelial Function in Health and Disease. Physiol Rev. 2018;98(4):1983-2023 DOI: 10.1152/physrev.00054.2017.

108. Ohtani N, Kawada N. Role of the Gut-Liver Axis in Liver Inflammation, Fibrosis, and Cancer: A Special Focus on the Gut Microbiota Relationship. Hepatol Commun. 2019;3(4):456-70 DOI: 10.1002/ hep4.1331.

109. Dawson PA. Roles of Ileal ASBT and OSTalpha-OSTbeta in Regulating Bile Acid Signaling. Dig Dis. 2017;35(3):261-6 DOI: 10.1159/000450987.

110. Zhou M, Learned RM, Rossi SJ, DePaoli AM, Tian H, Ling L. Engineered fibroblast growth factor 19 reduces liver injury and resolves sclerosing cholangitis in Mdr2-deficient mice. Hepatology. 2016;63(3):914-29 DOI: 10.1002/hep.28257.

111. Halpern MD, Dvorak B. Does abnormal bile acid metabolism contribute to NEC? Semin Perinatol. 2008;32(2):114-21 DOI: 10.1053/j.semperi.2008.01.005.

112. Cherrington NJ, Estrada TE, Frisk HA, Canet MJ, Hardwick RN, Dvorak B, Lux K, Halpern MD. The hepatic bile acid transporters Ntcp and Mrp2 are downregulated in experimental necrotizing enterocolitis. Am J Physiol Gastrointest Liver Physiol. 2013;304(1):G48-56 DOI: 10.1152/ ajpgi.00317.2012.

113. Hulzebos CV, van Zoonen AG, Hulscher JB, Schat TE, Kooi EM, Koehorst M, Boverhof R, Krabbe PF, Groen AK, Verkade HJ. Fecal Bile Salts and the Development of Necrotizing Enterocolitis in Preterm Infants. PLoS One. 2017;12(1):e0168633 DOI: 10.1371/journal.pone.0168633.

114. Halpern MD, Holubec H, Saunders TA, Dvorak K, Clark JA, Doelle SM, Ballatori N, Dvorak B. Bile acids induce ileal damage during experimental necrotizing enterocolitis. Gastroenterology. 2006;130(2):359-72 DOI: 10.1053/j.gastro.2005.10.023.

115. Halpern MD, Weitkamp JH, Mount Patrick SK, Dobrenen HJ, Khailova L, Correa H, Dvorak B. Apical sodium-dependent bile acid transporter upregulation is associated with necrotizing enterocolitis. Am J Physiol Gastrointest Liver Physiol. 2010;299(3):G623-31 DOI: 10.1152/ajpgi.00242.2010.

116.Zorn AM, Wells JM. Vertebrate endoderm development and organ formation. Annu Rev Cell Dev Biol. 2009;25:221-51 DOI: 10.1146/annurev.cellbio.042308.113344.

117. Brandl K, Kumar V, Eckmann L. Gut-liver axis at the frontier of host-microbial interactions. Am J Physiol Gastrointest Liver Physiol. 2017;312(5):G413-G9 DOI: 10.1152/ajpgi.00361.2016. 
118. Fagarasan S, Muramatsu M, Suzuki K, Nagaoka H, Hiai H, Honjo T. Critical roles of activationinduced cytidine deaminase in the homeostasis of gut flora. Science. 2002;298(5597):1424-7 DOI: 10.1126/science.1077336.

119. Alverdy J, Chi HS, Sheldon GF. The effect of parenteral nutrition on gastrointestinal immunity. The importance of enteral stimulation. Ann Surg. 1985;202(6):681-4 DOI: 10.1097/00000658198512000-00003.

120. Kramer BW. Chorioamnionitis-newideas from experimental models. Neonatology. 2011;99(4):3205 DOI: 10.1159/000326620.

121. Wolfs TG, Jellema RK, Turrisi G, Becucci E, Buonocore G, Kramer BW. Inflammation-inducedimmune suppression of the fetus: a potential link between chorioamnionitis and postnatal early onset sepsis. J Matern Fetal Neonatal Med. 2012;25 Suppl 1:8-11 DOI: 10.3109/14767058.2012.664447.

122. Kataoka S, Yamada T, Chou K, Nishida R, Morikawa M, Minami M, Yamada H, Sakuragi N, Minakami H. Association between preterm birth and vaginal colonization by mycoplasmas in early pregnancy. J Clin Microbiol. 2006;44(1):51-5 DOI: 10.1128/JCM.44.1.51-55.2006.

123. Sweeney EL, Dando SJ, Kallapur SG, Knox CL. The Human Ureaplasma Species as Causative Agents of Chorioamnionitis. Clin Microbiol Rev. 2017;30(1):349-79 DOI: 10.1128/CMR.00091-16.

124. Okogbule-Wonodi AC, Gross GW, Sun CC, Agthe AG, Xiao L, Waites KB, Viscardi RM. Necrotizing enterocolitis is associated with ureaplasma colonization in preterm infants. Pediatr Res. 2011;69(5 Pt 1):442-7 DOI: 10.1203/PDR.0b013e3182111827.

125. Viscardi RM. Ureaplasma species: role in neonatal morbidities and outcomes. Arch Dis Child Fetal Neonatal Ed. 2014;99(1):F87-92 DOI: 10.1136/archdischild-2012-303351.

126. Plat J, Hendrikx T, Bieghs V, Jeurissen ML, Walenbergh SM, van Gorp PJ, De Smet E, Konings M, Vreugdenhil AC, Guichot YD, Rensen SS, Buurman WA, Greve JW, Lutjohann D, Mensink RP, ShiriSverdlov R. Protective role of plant sterol and stanol esters in liver inflammation: insights from mice and humans. PLoS One. 2014;9(10):e110758 DOI: 10.1371/journal.pone.0110758. 



\section{Chronic intra-uterine Ureaplasma parvum infection induces injury of the enteric nervous system in ovine fetuses}

C. Heymans, I.H. de Lange, M.C. Hütten, K. Lenaerts, N.J.E. de Ruijter, L.C.G.A. Kessels, G. Rademakers, V. Melotte, W. Boesmans, M. Saito, H. Usuda, S.J. Stock, O.B. Spiller, M.L. Beeton, M.S. Payne, B.W. Kramer, J.P Newnham, A.H. Jobe, M.W. Kemp, W.G. van Gemert, T.G.A.M. Wolfs 


\section{Abstract}

\section{Background}

Chorioamnionitis, inflammation of the fetal membranes during pregnancy, is often caused by intra-amniotic (IA) infection with single or multiple microbes. Chorioamnionitis can be either acute or chronic, and is associated with adverse postnatal outcomes of the intestine, including necrotizing enterocolitis (NEC). Neonates with NEC have structural and functional damage to the intestinal mucosa and the enteric nervous system (ENS), with loss of enteric neurons and glial cells. Yet, the impact of acute, chronic or repetitive antenatal inflammatory stimuli on the development of the intestinal mucosa and ENS has not been studied. The aim of this study is therefore to investigate the effect of acute, chronic and repetitive microbial exposure on the intestinal mucosa, submucosa and ENS in premature lambs.

\section{Materials and Methods}

A sheep model of pregnancy was used in which the ileal mucosa, submucosa and ENS were assessed following IA exposure to lipopolysaccharide (LPS) for 2 or 7 days (acute), Ureaplasma parvum (UP) for 42 days (chronic) or repetitive microbial exposure (42 days UP with 2 or 7 days LPS).

\section{Results}

IA LPS exposure for 7 days or IA UP exposure for 42 days caused intestinal injury and inflammation in the mucosal and submucosal layer of the gut. Repetitive microbial exposure did not further aggravate injury of the terminal ileum. Chronic IA UP exposure caused significant structural ENS alterations characterized by loss of PGP9.5 and S100 $\beta$ immunoreactivity whereas these changes were not found after re-exposure of chronic UP-exposed fetuses to LPS for 2 or 7 days.

\section{Conclusion}

The in utero loss of PGP9.5 and S100 $\beta$ immunoreactivity following chronic UP exposure corresponds with intestinal changes in neonates with NEC, and may therefore form a novel mechanistic explanation for the association of chorioamnionitis and NEC. 


\section{Introduction}

Preterm birth is a common and major worldwide health issue, contributing to significant neonatal morbidity and mortality (1). Around one in every ten births are preterm, accounting for approximately 15 million premature newborns each year (2). Due to complications, over one million premature newborns will die shortly after birth (3, 4). Chorioamnionitis, defined as inflammatory cell infiltration of fetal membranes, is frequently associated with preterm birth and typically occurs due to an ascending bacterial infection (5-7) that can be acute or chronic (8). Intra-uterine exposure of preterm infants to chorioamnionitis is associated with an increased risk of adverse neonatal outcomes $(9,10)$, including necrotizing enterocolitis (NEC) $(9,11,12)$. Adverse gastrointestinal outcomes have been associated with both systemic fetal inflammatory response syndrome (FIRS) and direct exposure of the gut to swallowed infected amniotic fluid $(11,13,14)$. Chorioamnionitis can occur with intact membranes, which is common for genital mycoplasmas, such as Ureaplasma species (spp.), present in the lower genital tract of women $(6,15)$. The Ureaplasma spp. can cause chronic chorioamnionitis that does not evoke a maternal response, but is still associated with adverse fetal outcomes (16). In an experimental large animal model, we previously showed that an Ureaplasma parvum (UP) serovar 3 infection up to 14 days prior to delivery causes fetal gut inflammation with damaged villus epithelium, gut barrier loss and severe villus atrophy (17).

The injury caused by intra-uterine Ureaplasma spp. exposure might derive from the direct inflammatory reaction, as well as from potential interactions with other inflammatory stimuli. Chorioamnionitis is often polymicrobial, as over $65 \%$ of positive amniotic fluid cultures lead to the identification of two or more pathogens (7). In this context, we previously showed that cerebral and lung immune activation following intra-amniotic (IA) lipopolysaccharide (LPS) exposure was prevented when these animals were chronically pre-exposed to UP serovar $3(18,19)$. This illustrates that interactions between different microbes can occur, leading to organ-specific sensitization or preconditioning.

The enteric nervous system (ENS) consists of enteric neurons and glial cells, autonomously regulates gastrointestinal activity (i.e. secretion, absorption, and motility), and contributes to gut integrity (20). The formation of the ENS requires coordinated migration, proliferation and differentiation of neural crest progenitors, directed neurite growth, and establishment of a network of interconnected neurons and glia $(21,22)$. Although these processes mostly occur in utero, an important part of ENS development takes place postnatally $(23,24)$. Neonates with NEC have structural and functional damage of the submucosal and myenteric plexus, including loss of enteric neurons and glial cells (2527). The involvement of chorioamnionitis in the induction of adverse intestinal outcomes including NEC combined with the presence of ENS abnormalities in NEC, prompted us to study the impact of an antenatal infection on the ENS.

The aim of this study was therefore to investigate the effect of acute IA exposure to LPS and chronic exposure to UP on the intestinal mucosa and ENS in fetal lambs using a wellestablished sheep model of chorioamnionitis. In addition, we investigated the potential 
interactions of repetitive IA microbial stimuli by acute exposure to LPS in ovine fetuses that were chronically pre-exposed to UP.

\section{Materials and Methods}

\section{Animal model and experimental procedures}

All experiments were approved by the animal ethics committee of the University of Western Australia (Perth, Australia).

The animal model and experimental procedures were previously described (18). Briefly, 39 date-mated merino ewes were randomly assigned to six different groups of between five and eight animals, to receive IA injections under ultrasound guidance. Verification of the IA injections was done by amniotic fluid electrolyte analysis. The date-mated pregnant ewes received an IA injection of an in vitro cultured strain HPA5 of UP serovar $3\left(2 \times 10^{5}\right.$ color-changing units [CCU]) (28) 42 days prior to delivery (at 82 days of gestation, which corresponds to the second trimester in humans), or E. coli-derived LPS (O55:B5; Merck, Darmstadt, Germany), $10 \mathrm{mg}$ in two $\mathrm{mL}$ of saline, 2 days or 7 days prior to delivery (at respectively 122 and 117 days of gestation). Previously, we have shown that the half-life time of LPS in the amniotic fluid is relatively long (1.7 days) and that the LPS amount is higher than the essential threshold of $1 \mathrm{mg}$ for at least 5 days $(29,30)$. Chronic sustained UP infection was confirmed by positive culture of amniocentesis samples at intermediate time points and sterile amniotic fluid samples collected at caesarean delivery as previously described (31). Two or 7 days LPS exposure (prior to caesarean delivery) represents an acute inflammatory challenge. To evaluate the combined effect between these inflammatory modalities, a subgroup of chronically UP infected ewes received IA LPS at 35 and 40 days post UP infection (i.e. 7 or 2 days LPS exposure prior to delivery following 42 days of UP infection). A group receiving IA injections of sterile saline ( 2 or 7 days prior to delivery; respectively 6 and 2 animals which were pooled) served as controls (figure 1). Fetuses were surgically delivered at $124 \pm 2$ days of gestational age (term gestation in sheep $=150$ days), equivalent of approximately 30 weeks of human gestation. After delivery, fetuses were euthanized with intravenous pentobarbital ( $100 \mathrm{mg} / \mathrm{kg}$ ). For this experiment, fetuses of both sexes were used. 


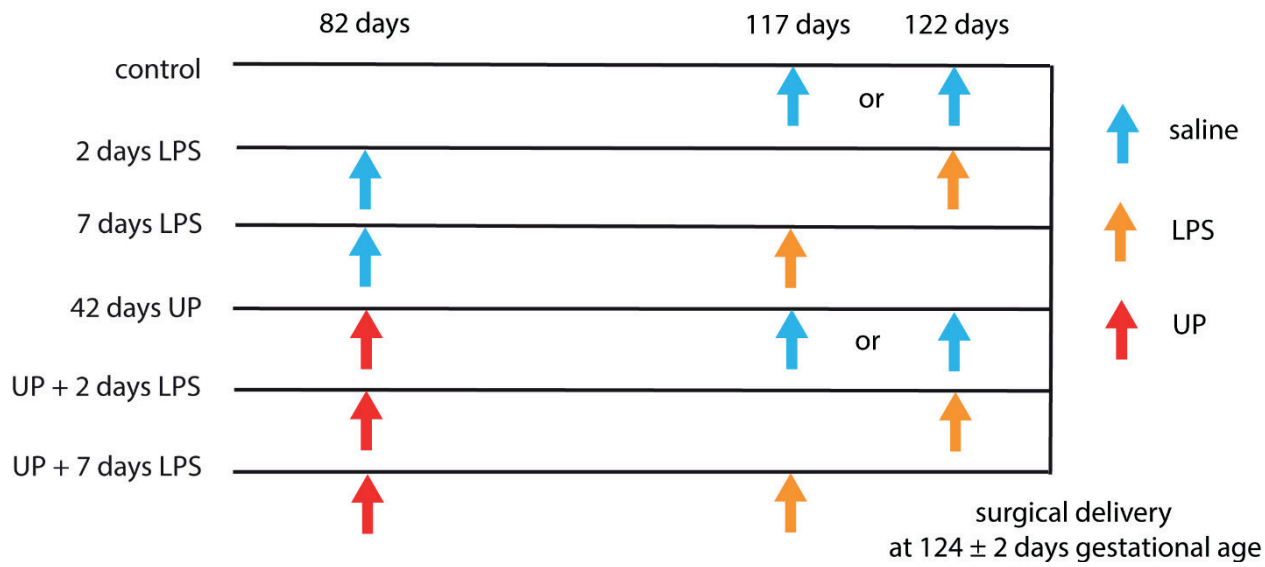

Figure 1. Different intervention study groups. All injections were delivered by ultrasound-guided amniocentesis. Timing shown in gestational days.

\section{Sampling}

During necropsy, blood and terminal ileum samples were collected. lleum samples were fixed in $10 \%$ formalin and embedded in paraffin, or snap frozen in liquid nitrogen. Where insufficient paraffin-embedded material was available for study, additional material was generated by paraffin embedding snap frozen tissue samples: frozen tissue blocks were defrosted, fixed in $4 \%$ formaldehyde at room temperature overnight and transferred to $70 \%$ ethanol prior to embedding in paraffin with the use of a vacuum infiltration processor.

\section{Antibodies}

The following antibodies were used for immunohistochemistry: polyclonal rabbit antimyeloperoxidase ([MPO]; A0398, Dakocytomation, Glostrup, Denmark) for identification of neutrophils, polyclonal rabbit anti- cluster of differentiation 3 ([CD3]; A0452, Dakocytomation) for the detection of T cells, polyclonal rabbit anti-bovine protein gene product 9.5 ([PGP9.5]; Z5116, Dakocytomation) for the detection of enteric neurons, polyclonal rabbit anti-doublecortin (Ab18723, Abcam, Cambridge, UK) for the detection of immature neurons, polyclonal rabbit anti-glial fibrillary acidic protein ([GFAP]; Zo334, Dakocytomation) to detect enteric glial cell reactivity/activation (32) and polyclonal rabbit anti-S100 $\beta$ (PA5-16257, Invitrogen, Carlsbad, CA, USA) as a marker for enteric glial cells.

The following secondary antibodies were used: peroxidase-conjugated polyclonal goat anti-rabbit (111-035-045, Jackson, WestGrove, PA, USA) (MPO), peroxidase-conjugated polyclonal swine anti-rabbit (P0399, DakoCytomation) (doublecortin) and BrightVision+ Poly-HRP-Anti Mouse/Rabbit IgG Biotin-free (ImmunoLogic, Duiven, the Netherlands) (PGP9.5), and biotin conjugated polyclonal swine anti-rabbit (E0353, DakoCytomation) (CD3, GFAP, S100ß). 


\section{Immunohistochemistry}

Formalin-fixed terminal ileum was embedded in paraffin and $4 \mu \mathrm{m}$ sections were cut. After deparaffinization and rehydration, endogenous peroxidase activity was blocked with $0.3 \% \mathrm{H}_{2} \mathrm{O}_{2}$ diluted in phosphorylated buffer saline ([PBS]; $\mathrm{pH} 7.4$ ). Antigen retrieval was performed with citrate buffer for CD3, PGP9.5, doublecortin and S100 $\beta$ stainings. Nonspecific binding was blocked for 30 minutes at room temperature with $10 \%$ normal goat serum (NGS) in PBS (MPO), 5\% NGS in PBS (doublecortin), or 5\% bovine serum albumin (BSA) in PBS (CD3, GFAP and S100ß). For PGP9.5, non-specific binding was blocked for 10 minutes at room temperature with $20 \%$ fetal calf serum (FCS). Thereafter, sections were incubated with the primary antibody of interest and subsequently incubated with the respective secondary antibody. MPO, PGP9.5 and doublecortin were detected by using a peroxidase-conjugated secondary antibody and antibodies against CD3, GFAP and $S 100 \beta$ were detected with avidin-biotin complex (Vectastain Elite ABC kit, Bio-connect, Huissen, the Netherlands). Substrate staining was performed for MPO with 3-amino-9ethylcarbazole ([AEC]; Merck, Darmstadt, Germany). Immunoreactivity for CD3 and GFAP was detected by using nickel-DAB. Immunoreactivity for PGP9.5, doublecortin and S100 $\beta$ was detected by using DAB. Haematoxyline (MPO, PGP9.5, doublecortin and S100ß) or nuclear fast red (CD3 and GFAP) were used as counterstain for nuclei.

\section{Qualitative analysis of damage of the terminal ileum}

$H \& E$ slides were analyzed by two independent investigators blinded to the experimental set-up, to assess damage of the terminal ileum. A scoring system from 0 to 4 was used to describe the severity of histological injury. Scoring was as follows: 0 no damage, 1 disrupted epithelial lining, but no loss of enterocytes, 2 disrupted epithelial lining, mild enterocyte loss from the villus tips, 3 disrupted epithelial lining, moderate enterocyte loss from villus tips, some debris in the lumen and 4 abundant enterocyte loss from villus tips, abundant debris in the lumen and severe shedding of villus tips.

\section{Quantification of immunohistochemical stainings}

The stained tissue sections were scanned with the Ventana iScan HT slide scanner (Ventana Medical Systems, Oro Valley, AZ, USA). Of these images, viewed with Panoramic Viewer (version 1.15.4, 3DHISTECH, Budapest, Hungary), random images of regions of interest were taken (200x).

Two investigators blinded to the study groups counted the MPO-positive and CD3-positive cells in three to five non-overlapping high power fields in the mucosa and submucosa. The average MPO- and CD3-positive cells per area are reported for each animal. The percentage of area in the submucosal and myenteric ganglia positively stained for PGP9.5, doublecortin, GFAP and $\mathrm{S} 100 \beta$ was determined in five non-overlapping high power fields using Leica QWin Pro software (version 3.4.0, Leica Microsystems, Mannheim, Germany) by an investigator blinded to the study groups. Relative area staining was calculated by dividing the positively stained areas of the ganglia of the submucosal or myenteric plexus by the total area of the muscle layer. The data are expressed as fold increase over the control value. 


\section{RNA extraction and quantitative real-time PCR}

RNA was extracted from snap frozen terminal ileum tissue using TRI reagent (Invitrogen)/ chloroform extraction. Isolated RNA was DNase treated to remove possible contamination with genomic DNA by using the RQ1 RNase-Free DNase kit (Promega, Madison, WI, USA) and afterwards reverse transcribed into cDNA using oligo(dT)12-18 primers (Invitrogen) and Moloney murine leukemia virus (M-MLV) reverse transcriptase (Invitrogen). Quantitative real-time PCR (qPCR) reactions were performed with a LightCycler 480 Instrument (Roche Applied Science, Basel, Switzerland) using the SensiMix ${ }^{\mathrm{TM}}$ SYBR $^{\circledR}$ No-ROX Kit (Bioline, London, UK) for 45 cycles. mRNA levels of IL-1 $\beta$, IL-6, IL-10, tumor necrosis factor alpha (TNF-a) and interleukin-1 receptor-associated kinase 3 (IRAK3) were determined to assess inflammation of the terminal ileum. mRNA levels of neuronal nitric oxide synthase (nNOS) and choline acetyltransferase (CHAT) were determined to assess motility signaling functions of the ENS using LinRegPCR software (version 2016.0, Heart Failure Research Center, Academic Medical Center, Amsterdam, the Netherlands). The geometric mean of the mRNA levels of three reference genes (ribosomal protein S15 (RPS15), glyceraldehyde 3-phosphate dehydrogenase (GAPDH) and peptidylprolyl isomerase A (PPIA)) were calculated and used as a normalization factor. The data are expressed as fold increase over the control value. Primer sequences are shown in Table 1.

Table 1. Primer sequences

\begin{tabular}{|c|c|c|}
\hline Primer & Forward & Reverse \\
\hline RPS15 & 5'-CGAGATGGTGGGCAGCAT-3' & 5'-GCTTGATTTCCACCTGGTTGA-3' \\
\hline GAPDH & 5'-GGAAGCTCACTGGCATGGC-3' & 5'-CCTGCTTCACCACCTTCTTG-3' \\
\hline PPIA & 5'-TTATAAAGGTTCCTGCTTTCACAGAA-3' & 5'-ATGGACTTGCCACCAGTACCA-3' \\
\hline $\mathrm{IL}-1 \beta$ & 5'-AGAATGAGCTGTTATTTGAGGTTGATG-3' & 5'-GTGAGAAATCTGCAGCTGGATGT-3' \\
\hline IL-6 & 5'-ACATCGTCGACAAAATCTCTGCAA-3' & 5'-GCCAGTGTCTCCTTGCTGTTT-3' \\
\hline IL-10 & 5'-CATGGGCCTGACATCAAGGA-3' & 5'-CGGAGGGTCTTCAGCTTCTC-3' \\
\hline TNF-a & 5'-GCCGGAATACCTGGACTATGC-3' & 5'-CAGGGCGATGATCCCAAAGTAG-3' \\
\hline IRAK3 & 5'-AGTGTGTAGGTAACACAGCCC-3' & 5'-TGCTGGTCATGCTTATGGCA-3' \\
\hline nNOS & 5'-CGGCTTTGGGGGTTATCAGT-3' & 5'-TTGCCCCATTTCCACTCCTC-3' \\
\hline CHAT & 5'-CCGCTGGTATGACAAGTCCC-3' & 5'-GCTGGTCTTCACCATGTGCT-3' \\
\hline
\end{tabular}

\section{Data analysis}

Data are presented as median with interquartile range. Statistical analyses were performed using GraphPad Prism (version 6.01, GraphPad Software Inc., La Jolla, CA, USA). A nonparametric Kruskal-Wallis test followed by Dunn's post hoc test was used to analyze statistically significant group differences. Differences were considered statistically significant at $p<0.05$. Given the relatively small animal numbers per group, we also reported actual $p$-values between $p \geq 0.05$ and $p<0.10$ and interpreted these as potentially biologically relevant. This assumption will decrease the chance of a type II error, but increases the chance of a type l error. 


\section{Results}

Intestinal damage and inflammation in the terminal ileum due to chorioamnionitis There was a higher intestinal damage score for all experimental groups, compared to control ( $p<0.005$ for 7d LPS group, $p<0.05$ for $42 d$ UP group and $42 d$ UP $+7 d$ LPS group and $p=0.06$ for $42 d$ UP $+2 d$ LPS group all compared to control; figure 2), except for the animals exposed to 2 days of LPS. Pre-exposure with UP did not augment mucosal injury in the LPS-treated groups.

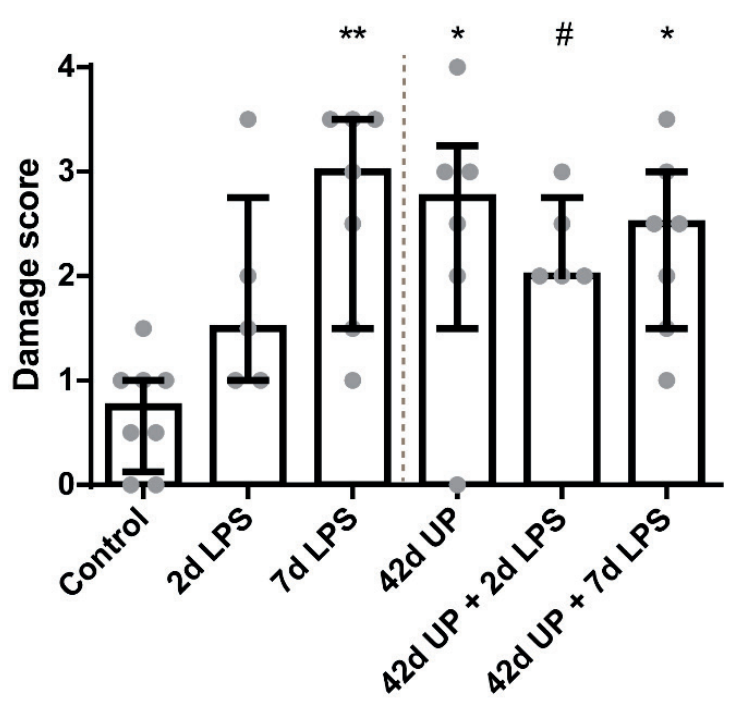

Figure 2. Increased mucosal injury in all groups, except for the animals exposed to 2 days LPS. \# $p=0.06,{ }^{*} p<0.05$ and ${ }^{* *} \mathrm{p}<0.005$ compared to control.

A statistically significant increase in MPO-positive cells was seen in the mucosa 7 days after LPS exposure, compared to control ( $p<0.05$; figure 3 ). Chronic UP infection also caused an elevation of mucosal MPO-positive cells, compared to control ( $p=0.08$; figure 3 ). Furthermore, combining these two inflammatory insults resulted in an increased mucosal MPO-positive cell count compared to control ( $p<0.005$; figure 3 ) and this experimental group tended to be increased when compared to the UP + two-day LPS-exposed group $(p=0.07$; figure 3). LPS exposure 2 days prior to delivery was insufficient to induce mucosal MPO-positive cell infiltration. Pre-exposure to UP in combination with LPS administration did not alter the number of mucosal MPO-positive cells, compared to LPS alone, both after 2 days and 7 days. 
A
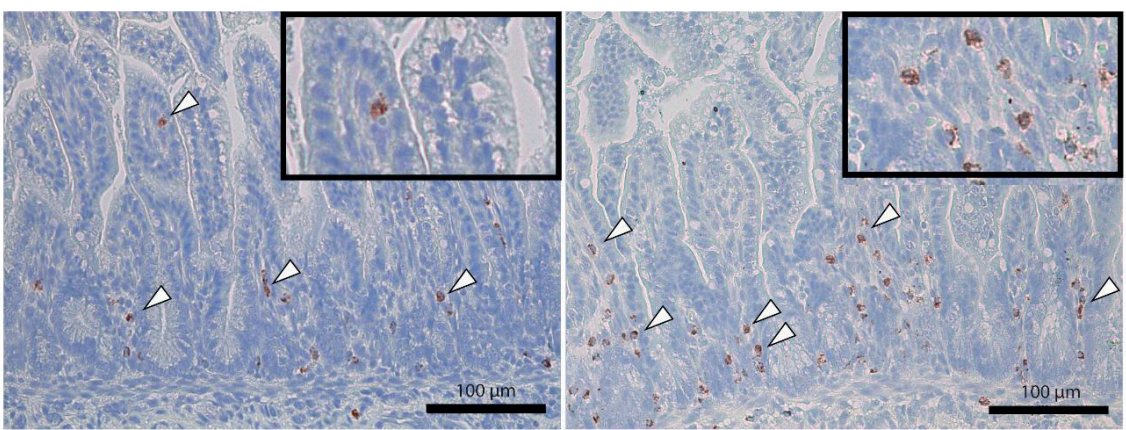

B
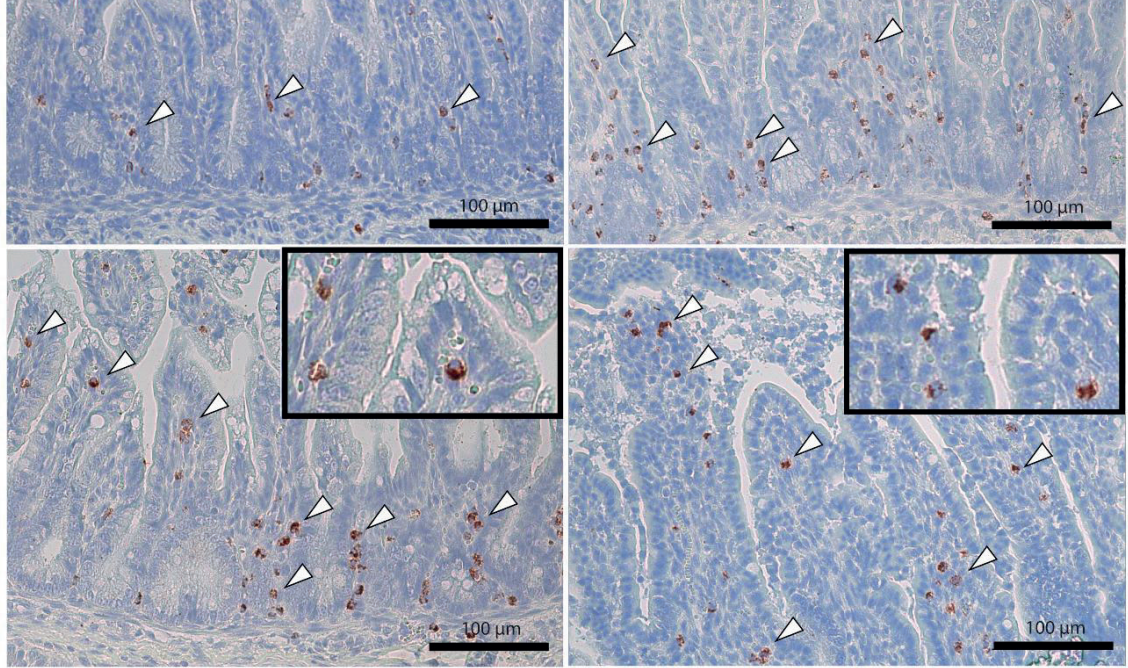

D

E

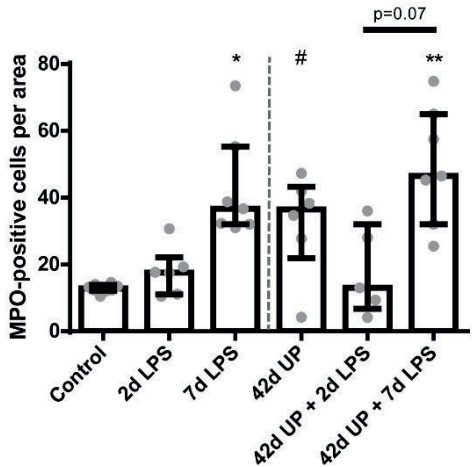

Figure 3. Representative images of mucosal neutrophil influx reflected by MPO-positive cell (indicated by white triangles) counts of the control (A), 7 days LPS (B), UP (C) and UP prior to 7 days LPS group (D). E: Increased MPO count in animals exposed to 7 days LPS, UP and UP prior to 7 days LPS. $\# p=0.08,{ }^{*} p<0.01,{ }^{* *} p<0.005$ compared to control. 
While chronic UP infection and acute LPS exposure 2 days pre-delivery did not have any effect on mucosal CD3-positive T cell presence, those animals receiving LPS 7 days predelivery (both uninfected and chronic UP infected groups) as well as chronic UP infected animals receiving LPS 2 days pre-delivery all showed apparent elevated levels of CD3positive $T$ cell infiltration (figure 4). However, the only comparison to achieve $p<0.05$ significance was that of uninfected and chronic UP infected animals receiving LPS 2 days pre-delivery (figure 4).
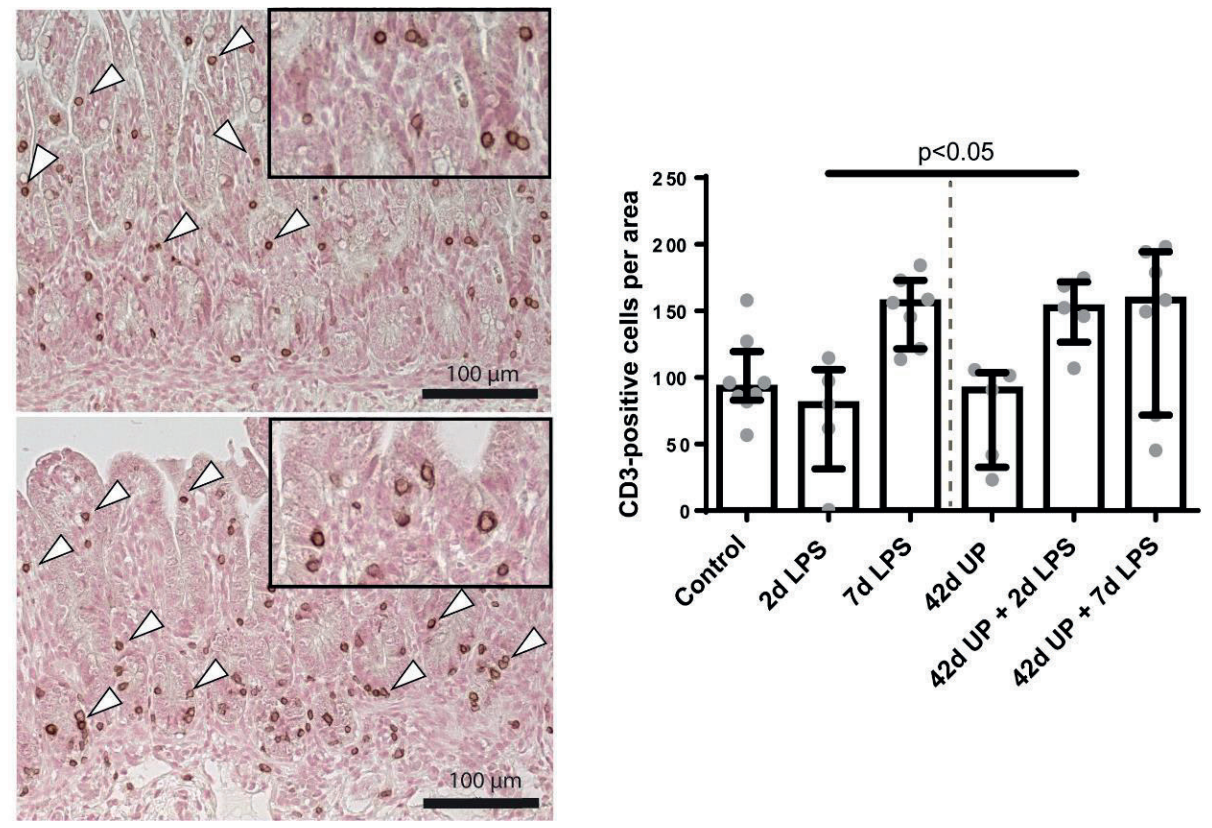

Figure 4. Representative images of mucosal $T$ cell influx reflected by CD3-positive cell (indicated by white triangles) counts of the control (A) and UP prior to two-day LPS (B). C: Increased CD3 count in animals exposed to UP prior to 2 days LPS compared to the 2 days LPS group. 
For investigation of submucosal inflammation, there was an increase of MPO-positive cells in the seven-day LPS group and submucosal MPO-positive cells tended to be increased in the chronic UP infection group, compared to control $(p<0.05$ and $p=0.06$; figure 5). Additional acute LPS insult ( 2 or 7 days pre-delivery) in chronic UP infected animals resulted in increased variability and loss of significance in the MPO cell infiltration compared to 2 or 7 days of LPS alone.
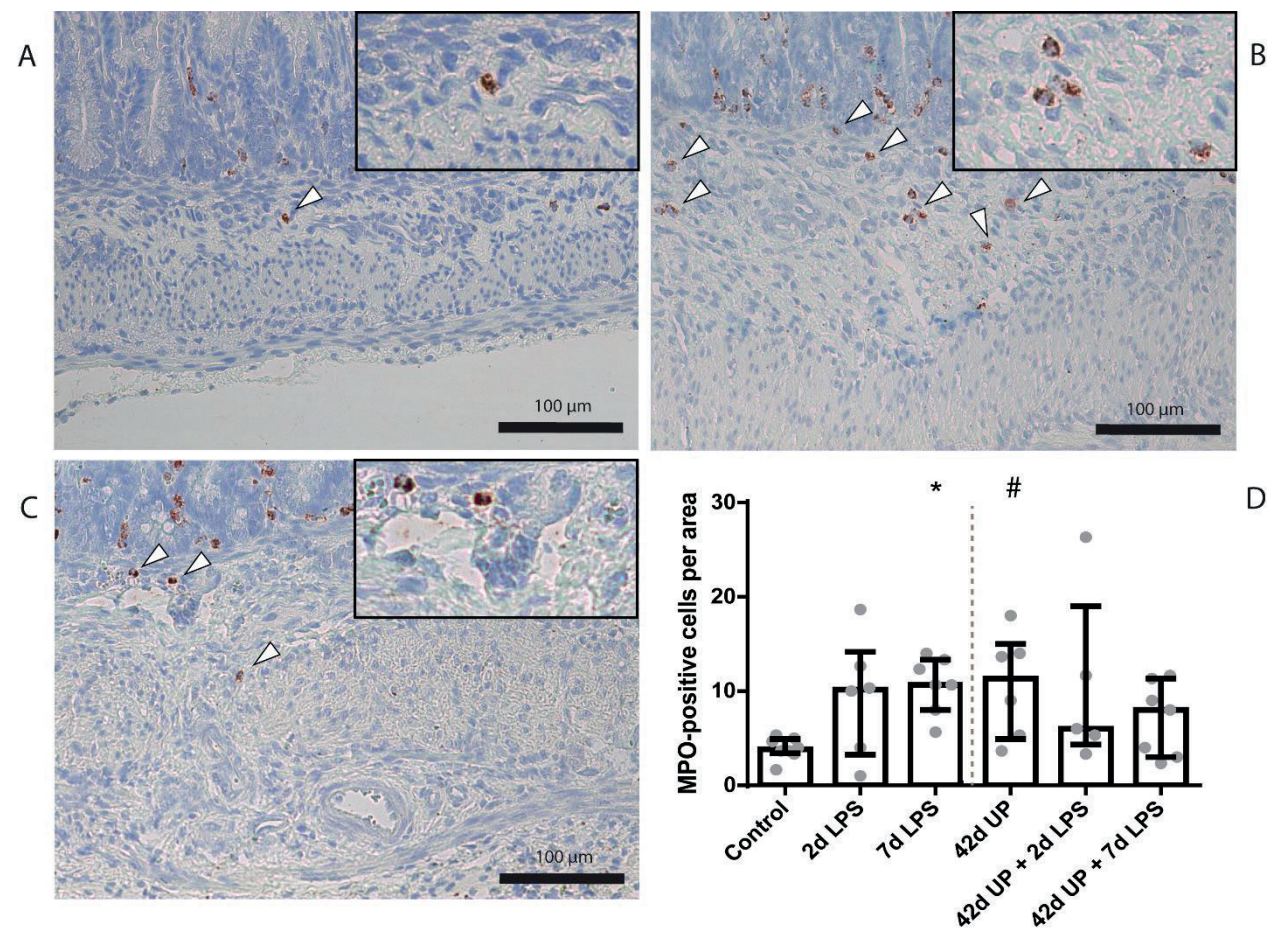

Figure 5. Representative images of submucosal neutrophil influx reflected by MPO-positive cell (indicated by white triangles) counts of the control (A), 7 days LPS (B) and UP (C). D: Increased MPO count in animals exposed to 7 days LPS and UP. \# $p=0.06,{ }^{*} p<0.05$ compared to control. 
The greatest increase of submucosal CD3-positive cells was observed in two-day LPSexposed chronic UP infected animals, which was significantly increased compared to control or acute 2 day LPS stimulation alone (both $p<0.05$; figure 6 ) and appeared more potent than in chronic UP infected animals receiving LPS at 7 days pre-delivery $(p=0.08$; figure 6).

A
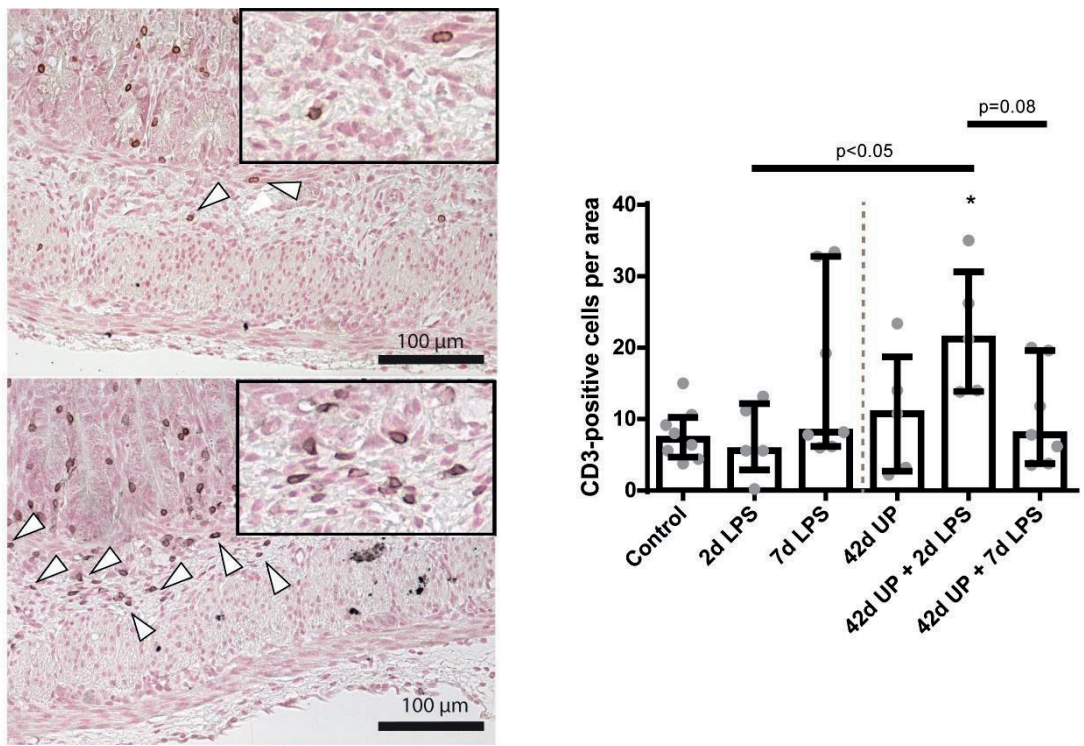

Figure 6. Representative images of submucosal T cell influx reflected by CD3-positive cell (indicated by white triangles) counts of the control (A) and UP prior to 2 days LPS group (B). C: Increased CD3 count in animals exposed to UP prior to 2 days LPS. ${ }^{*} \mathrm{p}<0.05$ compared to control.

Examination of underlying cytokine levels revealed increased IL-1 $\beta$ mRNA levels only in uninfected or chronic UP infected animals when LPS was administered 2 days pre-delivery ( $p<0.05$ compared to $7 d$ LPS group; and $p<0.01$ compared to $42 d$ UP group, $p<0.05$ compared to $42 d$ UP $+7 d$ LPS group and $p<0.05$ compared to control respectively; figure $7 A)$. Whereas IL-1 $\beta$ mRNA levels had dropped to baseline again if LPS was administered 7 days pre-delivery (figure 7A). IL-6 and IL-10 mRNA levels were not altered (data not shown) and the only group showing apparent TNF-a mRNA level elevation were the chronic UP infected animals additionally receiving LPS 2 days pre-delivery $(p=0.07$; figure 7B). 
IRAK3 mRNA levels were increased significantly only in animals exposed to 2 days of LPS alone compared to control ( $p<0.05$; figure $7 C)$.

A

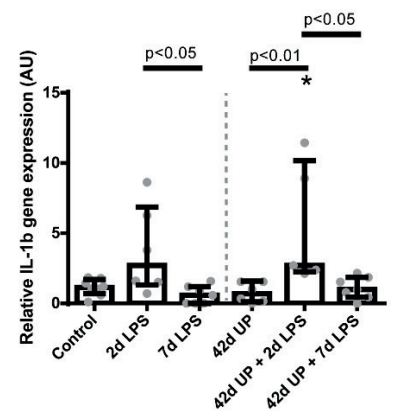

B

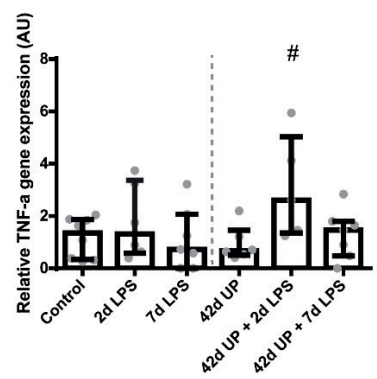

C

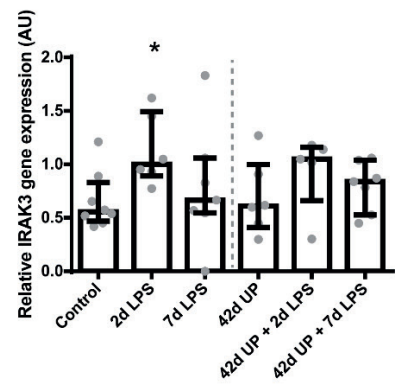

Figure 7. Relative mRNA levels of IL-1 $\beta$ (A), TNF- $\alpha$ (B) and IRAK3 (C) in arbitrary unit (AU). A: Increased IL- $1 \beta$ mRNA level in animals exposed to 2 days LPS and UP prior to 2 days LPS. ${ }^{*} p<0.05$ compared to control. B: Increased TNF- $a$ mRNA level in animals exposed to UP prior to 2 days LPS. \# $p=0.07$ compared to control. C: Increased IRAK3 mRNA level in animals exposed to 2 days LPS. * $p<0.05$ compared to control. 


\section{ENS alterations in the terminal ileum due to chronic IA UP exposure}

The PGP9.5-positive surface area in the submucosal plexus tended to be decreased in animals chronically infected for 42 days with UP, compared to control ( $p=0.08$; figure 8). Similarly, chronic UP infected animals had a diminished PGP9.5-positive surface area in the myenteric plexus ( $p<0.05$; figure 8$)$.
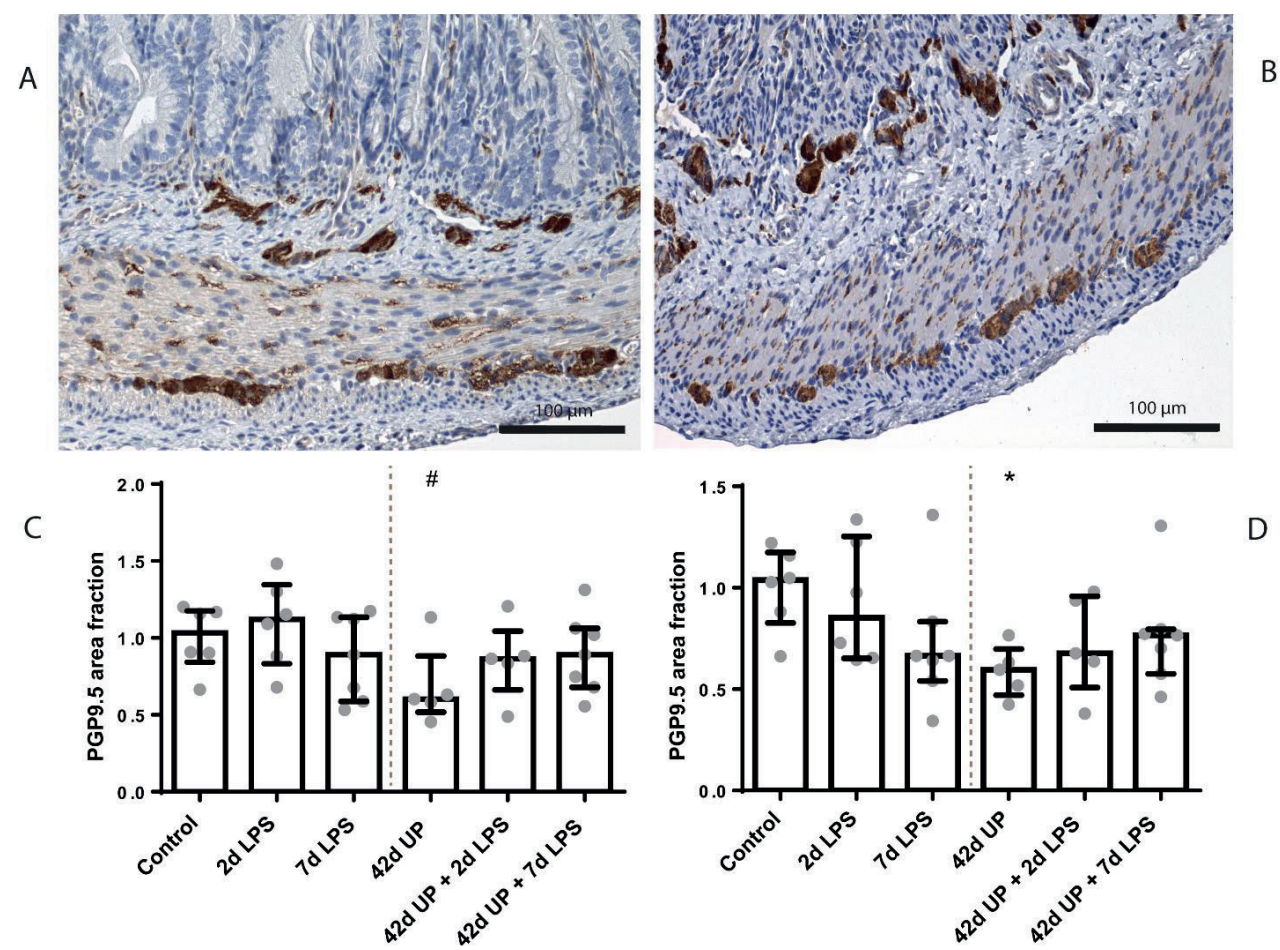

Figure 8. Representative images of PGP9.5 immunoreactivity in the submucosal and myenteric plexus of the control (A) and UP group (B). Area fraction of PGP9.5 in the submucosal plexus (C) and myenteric plexus (D) as fold increase over the control value. C: PGP9.5-positive surface area tended to be decreased in the submucosal plexus of animals exposed to UP. \# $p=0.08$ compared to control. D: Decreased PGP9.5-positive surface area in the myenteric plexus of animals exposed to UP. * $\mathrm{p}<0.05$ compared to control.

Doublecortin-positive surface areas were not altered in either the submucosal or the myenteric plexus (data not shown). 
In the submucosal plexus, the GFAP-positive surface area tended to be increased in groups receiving LPS either 2 or 7 days pre-delivery, compared to control $(p=0.07$ and $p=0.09$; figure 9), while in the myenteric plexus, the GFAP-positive surface area was only increased in the group receiving LPS 7 days pre-delivery, compared to control $(p<0.05$; figure 9). For both of these regions, concomitant chronic infection by UP appeared to mute these effects.

A

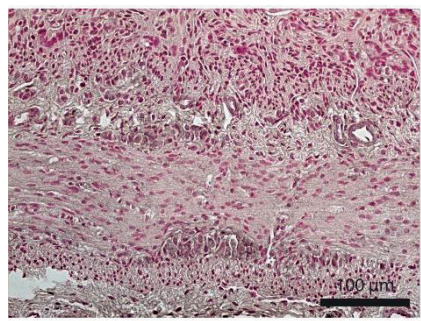

B
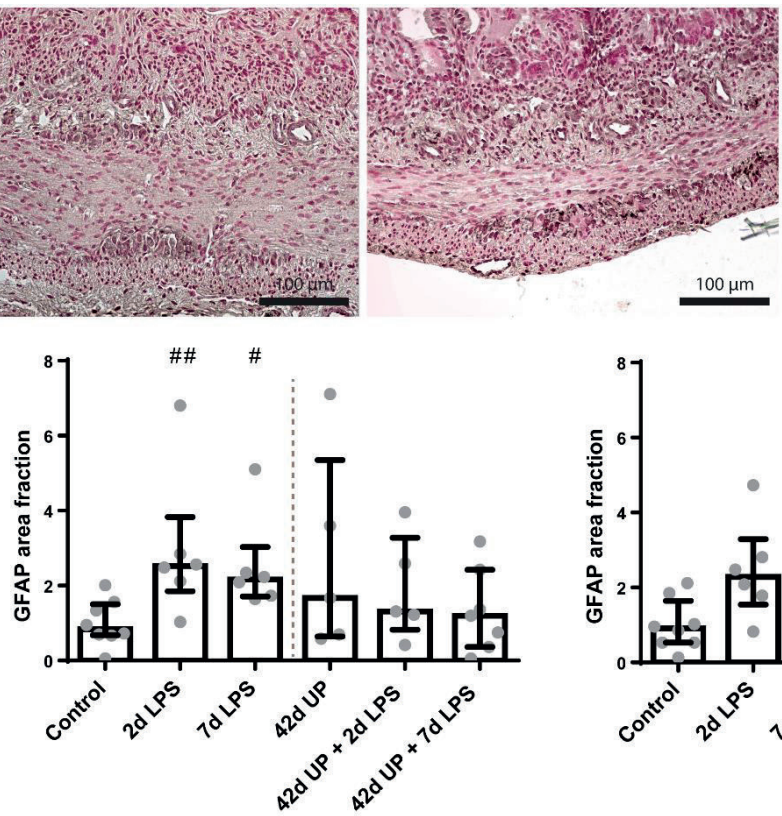

C
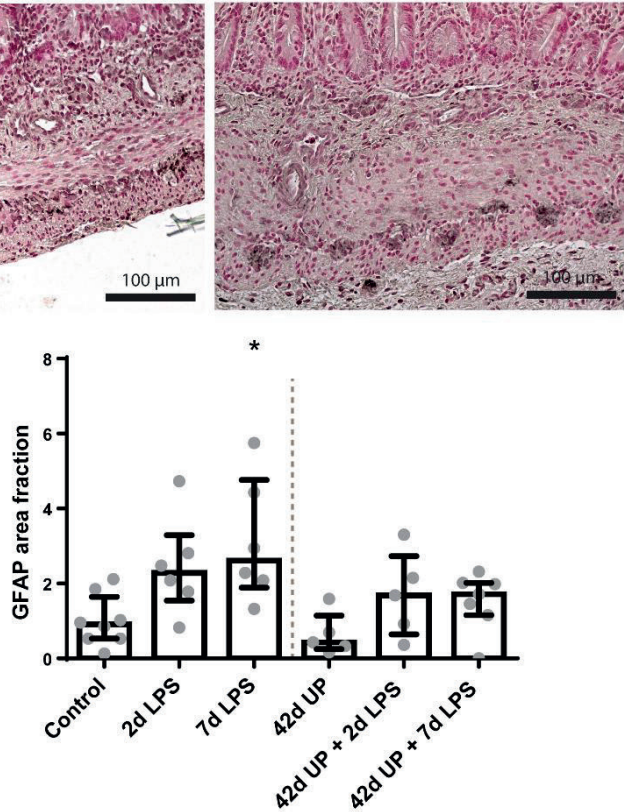

Figure 9. Representative images of GFAP immunoreactivity in the submucosal and myenteric plexus of the control (A), 2 days LPS (B) and 7 days LPS group (C). Area fraction of GFAP in the submucosal plexus (D) and myenteric plexus (E) as fold increase over the control value. D: GFAP-positive surface area tended to be increased in the submucosal plexus of animals exposed to 2 and 7 days LPS. \#\# $p=0.07$ and $\# p=0.09$ compared to control. E: Increased GFAP-positive surface area in the myenteric plexus of animals exposed to 7 days LPS. ${ }^{*} p<0.05$ compared to control. 
S100ß-positive surface areas were unaltered in the submucosal plexus for all conditions (data not shown), while in the myenteric plexus, the $\$ 100 \beta$-positive surface area was significantly decreased in the chronic UP infected group, compared to control $(p<0.05$; figure 10), but this effect appeared to be counteracted by acute stimulation by LPS at either 2 or 7 days pre-delivery.

A

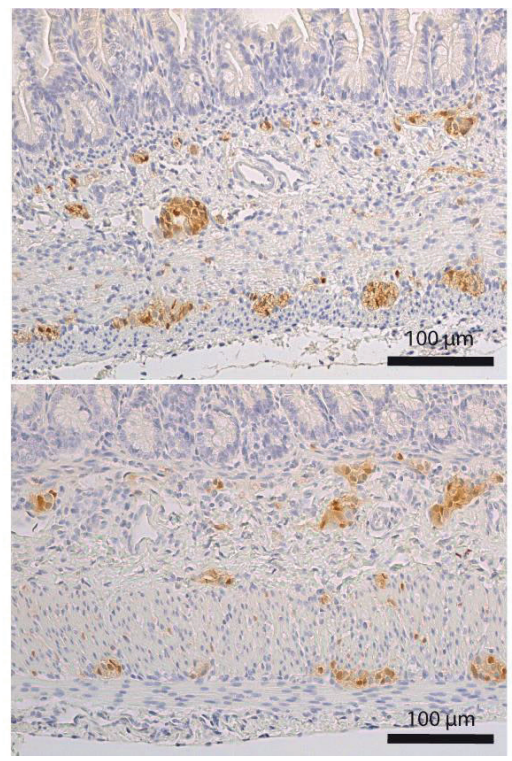

B

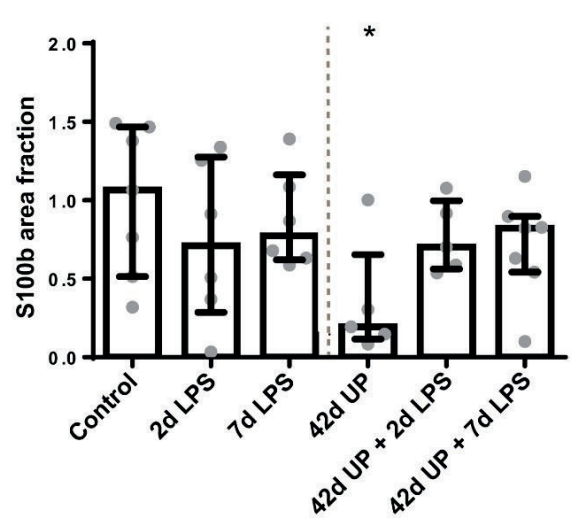

Figure 10. Representative images of $S 100 \beta$ immunoreactivity in the submucosal and myenteric plexus of the control (A) and UP group (B). Area fraction of $S 100 \beta$ in the myenteric plexus (C) as fold increase over the control value. C: S100 $\beta$-positive surface area was decreased in the myenteric plexus of animals exposed to UP. ${ }^{*} p<0.05$ compared to control.

No differences in nNOS and CHAT expression were observed between the groups (data not shown). 


\section{Discussion}

In this study, we investigated the effect of acute, chronic and combined microbial exposure as an antenatal infectious trigger (chorioamnionitis) on the mucosa, submucosa and ENS of the terminal ileum of premature lambs.

Both acute LPS and chronic UP exposure caused mucosal inflammation and injury to the terminal ileum. Although the inflammatory signature differed between these groups, mucosal injury was not aggravated in the combined exposure groups. Prenatal IA exposure to 7 days of LPS and to chronic 42 day infection by UP both provoked an influx of neutrophils (MPO-positive cells) in the intestine. By contrast, T cell (CD3-positive cells) numbers remained unaltered in the chronic UP and 2 day LPS groups compared to control group, but were increased in the UP +2 day LPS-exposed animals, indicating a potential synergistic effect of both inflammatory stimuli in inducing an adaptive mucosal immune response. We observed a similar effect in the submucosa: while either LPS exposure or UP infection induced innate immune changes in the ileum, $T$ cell alterations only occurred in the presence of combined UP and LPS exposure. Based on the current findings, we can only speculate on the mechanism behind this synergistic effect. In previous in vitro studies, signaling via Toll-like receptor (TLR) 1,2 and 6 by Ureaplasma spss. increased LPSmediated inflammation $(33,34)$. Additionally, TNF-a mRNA levels tended to be increased in the UP infected animals receiving LPS 2 days pre-delivery, while TNF-a levels were not increased upon single exposure to either UP or LPS alone. By contrast, no synergistic effect of UP and LPS exposure on intestinal IL-1 $\beta$ mRNA levels was found in the current study. This is supported by our IRAK3 mRNA findings, a negative regulator of TLR signaling (35), which remained unaltered in combined exposure of UP infected animals to LPS. Based on these joined findings, it is tempting to speculate that UP + LPS induced upregulation of cell adhesion molecules, and consequently temporarily increased diapedesis, could at least in part be responsible for the observed increase in CD3-positive cells. The latter suggestion is supported by previous in vitro findings showing enhanced endothelial protein expression of the cell adhesion molecule CXCR7 following co-incubation with LPS and UP, which was not observed in independently UP or LPS-exposed cells (36).

Interestingly, other ovine studies have reported a suppressive immune effect in the premature lung and brain after chronic UP exposure prior to acute LPS exposure (18, 19). Taken together, these data show that cells might be sensitized, preconditioned or remain unaffected following chronic UP infection, indicating organ dependent responses. The mechanisms responsible for organ specific effects of a second hit chorioamnionitis remain to be elucidated.

The ENS closely interacts with intestinal immune cells (37). As such, ENS alterations can both result from intestinal inflammation and modulate it $(38,39)$. In this study, the most evident signs of ENS alterations were seen after chronic UP infection, which caused a reduced PGP9.5-positive surface area in both plexuses, likely representing a loss of enteric neurons. Alternatively, this might represent a loss of PGP9.5 immunoreactivity of enteric neurons. The doublecortin-positive (immature neurons) surface area in chronically UP-infected animals was unchanged, indicating that a decrease of mature neurons is 
responsible for the observed neuronal cell loss. As the period between 10 and 18 weeks of gestation is considered to be of paramount importance for both morphological and functional maturation of the ENS $(40,41)$, one might assume that the timing of our inflammatory challenge during this vulnerable second trimester is the key determinant for the observed effects, rather than the nature of the microbial trigger. The loss of enteric neurons in the myenteric plexus following chronic UP infection coincides with a reduced S100ß-positive surface area, suggesting a reduced number of enteric glial cells. However, a reduction of $S 100 \beta$ immunoreactivity within glial cells could be involved in the observed effect as well. Enteric glial cells are known to contribute to neuronal maintenance, survival and function (42). Interestingly, the $S 100 \beta$-positive surface area was less reduced in the groups exposed to an additional LPS challenge in combination with chronic UP infection and the median of the PGP9.5-positive surface area was higher in these groups. In support, previous studies have shown that enteric glial cells are capable of generating enteric neurons in response to injury $(43,44)$, indicating that glial cells could be the driving cells behind the loss or gain of neurons in our model. As a hallmark of their high level of cellular plasticity (45), enteric glia can respond to inflammatory cues and ENS damage by alternating their morphology and expression of key proteins such as GFAP, in a process similar to reactive astrogliosis $(46,47)$. In this study, GFAP immunoreactivity was increased in both plexuses in the LPS-exposed animals, indicating that a glial response is induced by intestinal inflammation (48). An enteric glial cell response was not detected in chronic UP infected animals, despite signs of intestinal inflammation, suggesting normalization of GFAP levels within this period. Interestingly, pre-conditioning through chronic UP infection prevented GFAP upregulation in response to the overlapping second challenge with LPS in the glial cells in both plexuses, as no altered GFAP immunoreactivity was seen following subsequent IA LPS exposure. Whether this is solely protective or can contribute to the ENS damage seen in chronic UP exposure is unclear, as activation of enteric glia in the context of intestinal inflammation has been described to be both destructive (49) and potentially neuroregenerative (50). We may conclude from the aforementioned findings that enteric glial cells are already able to react to inflammatory cues prenatally. Importantly, our results suggest that these cells play an important role in neuronal survival and neurogenesis in the intra-uterine setting.

At present, the postnatal consequences of the detected loss of mature neurons and glial cells following UP exposure in the second trimester remain unknown. A similar decrease in enteric neurons has been described in models of experimental colitis, which show that neuronal loss persists after recovery of inflammation (51) and is accompanied by decreased colonic motility (52). Based on these combined findings it is likely that the observed changes in utero will result in ENS dysfunction postnatally.

Interestingly, several studies describe intestinal changes in patients with acute NEC that are similar to those found after chronic UP infection, namely loss of both enteric neurons $(25-27,53)$ and glial cells $(25-27)$. Moreover, it has been suggested that ablation of enteric glial cells may be an upstream target of NEC pathology (54). A potential causal role of the ENS in NEC pathophysiology is further supported by a rat study in which increased NEC survival and intestinal motility was associated with improvement of ENS changes, 
including an increase in enteric neurons (27). Collectively, our findings form a novel mechanistic explanation for the reported association of chorioamnionitis and NEC.

A limitation of this study is that it only enables us to study the effects of UP and LPS exposure at one time point, preventing us from dissecting the role of the different inflammatory triggers (LPS and UP) of inflammation duration (acute and chronic). In addition, group sizes are small, which is an inherent shortcoming of the translational ovine model used.

In summary, an acute inflammatory stimulus with LPS or a chronic inflammatory stimulus with UP causes intestinal injury and inflammation in the mucosal and submucosal layers of the gut. Combined overlapping microbial exposure does not aggravate injury of the terminal ileum. Most importantly, chronic UP infection causes structural ENS alterations characterized by PGP9.5 and S100 $\beta$ immunoreactivity loss. Whether the observed ENS alterations result in functional abnormalities after birth remains to be elucidated. However, the observed changes in utero correspond with findings in neonates with NEC, which underlines the concept that NEC pathophysiology may already have its origin in utero. 


\section{References}

1. Murphy SL, Mathews TJ, Martin JA, Minkovitz CS, Strobino DM. Annual Summary of Vital Statistics: 2013-2014. Pediatrics. 2017;139(6) DOI: 10.1542/peds.2016-3239.

2. Howson CP, Kinney MV, McDougall L, Lawn JE, Born Too Soon Preterm Birth Action G. Born too soon: preterm birth matters. Reprod Health. 2013;10 Suppl 1:S1 DOI: 10.1186/1742-4755-10-S1S1.

3. Blencowe $\mathrm{H}$, Cousens $\mathrm{S}$. Addressing the challenge of neonatal mortality. Trop Med Int Health. 2013;18(3):303-12 DOI: 10.1111/tmi.12048.

4. Blencowe H, Cousens S, Oestergaard MZ, Chou D, Moller AB, Narwal R, Adler A, Vera Garcia C, Rohde S, Say L, Lawn JE. National, regional, and worldwide estimates of preterm birth rates in the year 2010 with time trends since 1990 for selected countries: a systematic analysis and implications. Lancet. 2012;379(9832):2162-72 DOI: 10.1016/S0140-6736(12)60820-4.

5. Galinsky R, Polglase GR, Hooper SB, Black MJ, Moss TJ. The consequences of chorioamnionitis: preterm birth and effects on development. J Pregnancy. 2013;2013:412831 DOI: $10.1155 / 2013 / 412831$.

6. Goldenberg RL, Hauth JC, Andrews WW. Intrauterine infection and preterm delivery. N Engl J Med. 2000;342(20):1500-7 DOI: 10.1056/NEJM200005183422007.

7. Tita AT, Andrews WW. Diagnosis and management of clinical chorioamnionitis. Clin Perinatol. 2010;37(2):339-54 DOI: 10.1016/j.clp.2010.02.003.

8. Peng CC, Chang JH, Lin HY, Cheng PJ, Su BH. Intrauterine inflammation, infection, or both (Triple I): A new concept for chorioamnionitis. Pediatr Neonatol. 2018;59(3):231-7 DOI: 10.1016/j. pedneo.2017.09.001.

9. SilwedelC, Speer CP, Glaser K. Ureaplasma-associated prenatal, perinatal, and neonatalmorbidities. Expert Rev Clin Immunol. 2017;13(11):1073-87 DOI: 10.1080/1744666x.2017.1381559.

10. Viscardi RM. Ureaplasma species: role in diseases of prematurity. Clin Perinatol. 2010;37(2):393409 DOI: 10.1016/j.clp.2009.12.003.

11. Been JV, Lievense S, Zimmermann LJ, Kramer BW, Wolfs TG. Chorioamnionitis as a risk factor for necrotizing enterocolitis: a systematic review and meta-analysis. J Pediatr. 2013;162(2):236-42 e2 DOI: 10.1016/j.jpeds.2012.07.012.

12. Okogbule-Wonodi AC, Gross GW, Sun CC, Agthe AG, Xiao L, Waites KB, Viscardi RM. Necrotizing enterocolitis is associated with ureaplasma colonization in preterm infants. Pediatr Res. 2011;69(5 Pt 1):442-7 DOI: 10.1203/PDR.0b013e3182111827.

13. Wolfs TG, Jellema RK, Turrisi G, Becucci E, Buonocore G, Kramer BW.Inflammation-induced immune suppression of the fetus: a potential link between chorioamnionitis and postnatal early onset sepsis. J Matern Fetal Neonatal Med. 2012;25 Suppl 1:8-11 DOI: 10.3109/14767058.2012.664447.

14. Wolfs TG, Kramer BW, Thuijls G, Kemp MW, Saito M, Willems MG, Senthamarai-Kannan P, Newnham JP, Jobe AH, Kallapur SG. Chorioamnionitis-induced fetal gut injury is mediated by direct gut exposure of inflammatory mediators or by lung inflammation. Am J Physiol Gastrointest Liver Physiol. 2014;306(5):G382-93 DOI: 10.1152/ajpgi.00260.2013.

15. Kataoka S, Yamada T, Chou K, Nishida R, Morikawa M, Minami M, Yamada H, Sakuragi N, Minakami H. Association between preterm birth and vaginal colonization by mycoplasmas in early pregnancy. J Clin Microbiol. 2006;44(1):51-5 DOI: 10.1128/JCM.44.1.51-55.2006.

16. Sweeney EL, Dando SJ, Kallapur SG, Knox CL. The Human Ureaplasma Species as Causative Agents of Chorioamnionitis. Clin Microbiol Rev. 2017;30(1):349-79 DOI: 10.1128/CMR.00091-16. 
17. Wolfs TG, Kallapur SG, Knox CL, Thuijls G, Nitsos I, Polglase GR, Collins JJ, Kroon E, Spierings J, Shroyer NF, Newnham JP, Jobe AH, Kramer BW. Antenatal ureaplasma infection impairs development of the fetal ovine gut in an IL-1-dependent manner. Mucosal Immunol. 2013;6(3):54756 DOI: 10.1038/mi.2012.97.

18. Gussenhoven R, Ophelders D, Kemp MW, Payne MS, Spiller OB, Beeton ML, Stock SJ, CilleroPastor B, Barre FPY, Heeren RMA, Kessels L, Stevens B, Rutten BP, Kallapur SG, Jobe AH, Kramer BW, Wolfs T. The Paradoxical Effects of Chronic Intra-Amniotic Ureaplasma parvum Exposure on Ovine Fetal Brain Development. Dev Neurosci. 2017;39(6):472-86 DOI: 10.1159/000479021.

19. Kallapur SG, Kramer BW, Knox CL, Berry CA, Collins JJ, Kemp MW, Nitsos I, Polglase GR, Robinson J, Hillman NH, Newnham JP, Chougnet C, Jobe AH. Chronic fetal exposure to Ureaplasma parvum suppresses innate immune responses in sheep. J Immunol. 2011;187(5):2688-95 DOI: 10.4049/ jimmunol.1100779.

20. Furness JB. The Enteric Nervous System. Blackwell Publishing, Oxford (2006). 2006 DOI: 10.1016/j. dld.2006.03.010.

21. Lake J,Heuckeroth RO. Entericnervous system development:migration, differentiation, and disease. Am J Physiol Gastrointest Liver Physiol. 2013;305(1):G1-24 DOI: 10.1152/ajpgi.00452.2012.

22. Rao M, Gershon MD. Enteric nervous system development: what could possibly go wrong? Nat Rev Neurosci. 2018;19(9):552-65 DOI: 10.1038/s41583-018-0041-0.

23. Burns AJ, Roberts RR, Bornstein JC, Young HM. Development of the enteric nervous system and its role in intestinal motility during fetal and early postnatal stages. Semin Pediatr Surg. 2009;18(4):196-205 DOI: 10.1053/j.sempedsurg.2009.07.001.

24. Hao MM, Foong JP, Bornstein JC, Li ZL, Vanden Berghe P, Boesmans W. Enteric nervous system assembly: Functional integration within the developing gut. Dev Biol. 2016;417(2):168-81 DOI: 10.1016/j.ydbio.2016.05.030.

25. Sigge W, Wedel T, Kuhnel W, Krammer HJ. Morphologic alterations of the enteric nervous system and deficiency of non-adrenergic non-cholinergic inhibitory innervation in neonatal necrotizing enterocolitis. Eur J Pediatr Surg. 1998;8(2):87-94 DOI: 10.1055/s-2008-1071128.

26. Wedel T, Krammer HJ, Kuhnel W, Sigge W. Alterations of the enteric nervous system in neonatal necrotizing enterocolitis revealed by whole-mount immunohistochemistry. Pediatr Pathol Lab Med. 1998;18(1):57-70.

27. Zhou Y, Yang J, Watkins DJ, Boomer LA, Matthews MA, Su Y, Besner GE. Enteric nervous system abnormalities are present in human necrotizing enterocolitis: potential neurotransplantation therapy. Stem Cell Res Ther. 2013;4(6):157- DOI: 10.1186/scrt387.

28. Moss TJ, Knox CL, Kallapur SG, Nitsos I, Theodoropoulos C, Newnham JP, Ikegami M, Jobe $\mathrm{AH}$. Experimental amniotic fluid infection in sheep: effects of Ureaplasma parvum serovars 3 and 6 on preterm or term fetal sheep. Am J Obstet Gynecol. 2008;198(1):122 e1-8 DOI: 10.1016/j. ajog.2007.06.065.

29. Kramer BW, Moss TJ, Willet KE, Newnham JP, Sly PD, Kallapur SG, Ikegami M, Jobe AH. Dose and time response after intraamniotic endotoxin in preterm lambs. Am J Respir Crit Care Med. 2001;164(6):982-8 DOI: 10.1164/ajrccm.164.6.2103061.

30. Newnham JP, Kallapur SG, Kramer BW, Moss TJ, Nitsos I, Ikegami M, Jobe AH. Betamethasone effects on chorioamnionitis induced by intra-amniotic endotoxin in sheep. Am J Obstet Gynecol. 2003;189(5):1458-66 DOI: 10.1067/s0002-9378(03)00758-0. 
31. Miura Y, Payne MS, Keelan JA, Noe A, Carter S, Watts R, Spiller OB, Jobe AH, Kallapur SG, Saito $M$, Stock SJ, Newnham JP, Kemp MW. Maternal intravenous treatment with either azithromycin or solithromycin clears Ureaplasma parvum from the amniotic fluid in an ovine model of intrauterine infection. Antimicrob Agents Chemother. 2014;58(9):5413-20 DOI: 10.1128/AAC.03187-14.

32. Rao M, Nelms BD, Dong L, Salinas-Rios V, Rutlin M, Gershon MD, Corfas G. Enteric glia express proteolipid protein 1 and are a transcriptionally unique population of glia in the mammalian nervous system. Glia. 2015;63(11):2040-57 DOI: 10.1002/glia.22876.

33. Manimtim WM, Hasday JD, Hester L, Fairchild KD, Lovchik JC, Viscardi RM. Ureaplasma urealyticum modulates endotoxin-induced cytokine release by human monocytes derived from preterm and term newborns and adults. Infect Immun. 2001;69(6):3906-15 DOI: 10.1128/IAI.69.6.39063915.2001.

34. Shimizu T, Kida Y, Kuwano K. Ureaplasma parvum lipoproteins, including MB antigen, activate NF-\{kappa\}B through TLR1, TLR2 and TLR6. Microbiology. 2008;154(Pt 5):1318-25 DOI: 10.1099/ mic.0.2007/016212-0.

35. Kobayashi K, Hernandez LD, Galan JE, Janeway CA, Jr., Medzhitov R, Flavell RA. IRAK-M is a negative regulator of Toll-like receptor signaling. Cell. 2002;110(2):191-202.

36. Silwedel C, Speer CP, Haarmann A, Fehrholz M, Claus H, Buttmann M, Glaser K. Novel insights into neuroinflammation: bacterial lipopolysaccharide, tumor necrosis factor alpha, and Ureaplasma species differentially modulate atypical chemokine receptor 3 responses in human brain microvascular endothelial cells. J Neuroinflammation. 2018;15(1):156 DOI: 10.1186/s12974-0181170-0.

37. Yoo BB, Mazmanian SK. The Enteric Network: Interactions between the Immune and Nervous Systems of the Gut. Immunity. 2017;46(6):910-26 DOI: 10.1016/j.immuni.2017.05.011.

38. Brierley SM, Linden DR. Neuroplasticity and dysfunction after gastrointestinal inflammation. Nat Rev Gastroenterol Hepatol. 2014;11(10):611-27 DOI: 10.1038/nrgastro.2014.103.

39. Margolis KG, Gershon MD. Enteric Neuronal Regulation of Intestinal Inflammation. Trends Neurosci. 2016;39(9):614-24 DOI: 10.1016/j.tins.2016.06.007.

40. Fekete E, Resch BA, Benedeczky I. Histochemical and ultrastructural features of the developing enteric nervous system of the human foetal small intestine. Histol Histopathol. 1995;10(1):127-34.

41. Fekete $E$, Bagyánszki M, Resch BA. Prenatal development of the myenteric plexus in the human fetal small intestine. Acta Biol Szeged [Internet]. 2000;44(1-4):3-19.

42. De Giorgio R, Giancola F, Boschetti E, Abdo H, Lardeux B, Neunlist M. Enteric glia and neuroprotection: basic and clinical aspects. Am J Physiol Gastrointest Liver Physiol. 2012;303(8):G887-93 DOI: 10.1152/ajpgi.00096.2012.

43. Joseph NM, He S, Quintana E, Kim YG, Nunez G, Morrison SJ. Enteric glia are multipotent in culture but primarily form glia in the adult rodent gut. J Clin Invest. 2011;121(9):3398-411 DOI: 10.1172/ JCl58186.

44. Laranjeira C, Sandgren K, Kessaris N, Richardson W, Potocnik A, Vanden Berghe P, Pachnis V. Glial cells in the mouse enteric nervous system can undergo neurogenesis in response to injury. J Clin Invest. 2011;121(9):3412-24 DOI: 10.1172/jci58200.

45. Boesmans W, Lasrado R, Vanden Berghe P, Pachnis V. Heterogeneity and phenotypic plasticity of glial cells in the mammalian enteric nervous system. Glia. 2015;63(2):229-41 DOI: 10.1002/ glia.22746.

46. Chow AK, Gulbransen BD. Potential roles of enteric glia in bridging neuroimmune communication in the gut. Am J Physiol Gastrointest Liver Physiol. 2017;312(2):G145-G52 DOI: 10.1152/ ajpgi.00384.2016. 
47. Rosenbaum C, Schick MA, Wollborn J, Heider A, Scholz CJ, Cecil A, Niesler B, Hirrlinger J, Walles $\mathrm{H}$, Metzger M. Activation of Myenteric Glia during Acute Inflammation In Vitro and In Vivo. PLoS One. 2016;11(3):e0151335 DOI: 10.1371/journal.pone.0151335.

48. Cirillo C, Sarnelli G, Turco F, Mango A, Grosso M, Aprea G, Masone S, Cuomo R. Proinflammatory stimuli activates human-derived enteroglial cells and induces autocrine nitric oxide production. Neurogastroenterol Motil. 2011;23(9):e372-82 DOI: 10.1111/j.1365-2982.2011.01748.x.

49. Brown IA, McClain JL, Watson RE, Patel BA, Gulbransen BD. Enteric glia mediate neuron death in colitis through purinergic pathways that require connexin-43 and nitric oxide. Cell Mol Gastroenterol Hepatol. 2016;2(1):77-91 DOI: 10.1016/j.jcmgh.2015.08.007.

50. Belkind-Gerson J, Graham HK, Reynolds J, Hotta R, Nagy N, Cheng L, Kamionek M, Shi HN, Aherne CM, Goldstein AM. Colitis promotes neuronal differentiation of Sox2+ and PLP1+ enteric cells. Sci Rep. 2017;7(1):2525 DOI: 10.1038/s41598-017-02890-y.

51. Linden DR, Couvrette JM, Ciolino A, McQuoid C, Blaszyk H, Sharkey KA, Mawe GM. Indiscriminate loss of myenteric neurones in the TNBS-inflamed guinea-pig distal colon. Neurogastroenterol Motil. 2005;17(5):751-60 DOI: 10.1111/j.1365-2982.2005.00703.x.

52. Boyer L, Ghoreishi M, Templeman V, Vallance BA, Buchan AM, Jevon G, Jacobson K. Myenteric plexus injury and apoptosis in experimental colitis. Auton Neurosci. 2005;117(1):41-53 DOI: 10.1016/j.autneu.2004.10.006.

53. Fagbemi AO, Torrente F, Puleston J, Lakhoo K, James S, Murch SH. Enteric neural disruption in necrotizing enterocolitis occurs in association with myenteric glial cell CCL20 expression. J Pediatr Gastroenterol Nutr. 2013;57(6):788-93 DOI: 10.1097/MPG.0b013e3182a86fd4.

54. Bush TG. Enteric glial cells. An upstream target for induction of necrotizing enterocolitis and Crohn's disease? Bioessays. 2002;24(2):130-40 DOI: 10.1002/bies.10039. 


$$
3
$$




\section{Chorioamnionitis induces enteric nervous system injury: effects of timing and inflammation in the ovine fetus}

C. Heymans*, I.H. de Lange*, K. Lenaerts, L.C.G.A. Kessels, M. Hadfoune, G. Rademakers, V. Melotte, W. Boesmans, B.W. Kramer, A.H. Jobe, M. Saito, M.W. Kemp, W.G. van Gemert, T.G.A.M. Wolfs

* Contributed equally 


\section{Abstract}

\section{Background}

Chorioamnionitis, inflammation of the chorion and amnion, which often results from intra-uterine infection, is associated with premature birth and contributes to significant neonatal morbidity and mortality, including necrotizing enterocolitis (NEC). Recently, we have shown that chronic chorioamnionitis is associated with significant structural enteric nervous system (ENS) abnormalities that may predispose to later NEC development. Understanding time point specific effects of an intra-amniotic (IA) infection on the ENS is important for further understanding the pathophysiological processes and for finding a window for optimal therapeutic strategies for an individual patient. The aim of this study was therefore to gain insight in the longitudinal effects of intra-uterine LPS exposure (ranging from 5 hours to 15 days before premature delivery) on the intestinal mucosa, submucosa, and ENS in fetal lambs by use of a well-established translational ovine chorioamnionitis model.

\section{Materials and Methods}

We used an ovine chorioamnionitis model to assess outcomes of the fetal ileal mucosa, submucosa and ENS following IA exposure to one dose of $10 \mathrm{mg}$ LPS for 5, 12 or 24 hours or $2,4,8$ or 15 days.

\section{Results}

Four days of IA LPS exposure causes a decreased PGP9.5- and S100 $\beta$-positive surface area in the myenteric plexus along with submucosal and mucosal intestinal inflammation that coincided with systemic inflammation. These changes were preceded by a glial cell reaction with early systemic and local gut inflammation. ENS changes and inflammation recovered 15 days after the IA LPS exposure.

\section{Conclusion}

The pattern of mucosal and submucosal inflammation, and ENS alterations in the fetus changed over time following IA LPS exposure. Although ENS damage seemed to recover after prolonged IA LPS exposure, additional postnatal inflammatory exposure, which a premature is likely to encounter, may further harm the ENS and influence functional outcome. In this context, 4 to 8 days of IA LPS exposure may form a period of increased ENS vulnerability and a potential window for optimal therapeutic strategies. 


\section{Introduction}

Chorioamnionitis, inflammation of the chorion and amnion during pregnancy, is associated with premature birth and contributes to significant neonatal morbidity and mortality (1-3). Chorioamnionitis typically results from a bacterial infection ascending through the birth canal (2). It is often clinically silent and therefore difficult to diagnose, but can nevertheless affect the developing fetus (4). As the fetus swallows the amniotic fluid (AF), the intestine is directly exposed to bacterial components and inflammatory cytokines present in the AF, which can consequently cause gut injury and inflammation (5). Moreover, during chorioamnionitis, the fetus can develop a fetal inflammatory response syndrome (FIRS), which is characterized by increased systemic interleukin 6 (IL-6) and interleukin 8 (IL-8) levels (6). FIRS is an independent risk factor for considerable neonatal morbidity, including the postnatal intestinal disease necrotizing enterocolitis (NEC) (4, 7). NEC has a high mortality of overall $25 \%$ with both significant short-term and longterm morbidity (8). Severe intestinal inflammation is associated with NEC and can result in gut necrosis $(8,9)$. Gut specimens from NEC patients contain alterations in the enteric nervous system (ENS) including a loss of neurons and glial cells (10-13). The ENS resides in the intestinal wall and consists of two plexuses; the submucosal and myenteric plexus (14). It operates autonomously and regulates diverse gastrointestinal functions such as motility, secretion, absorption and maintenance of gut integrity (14). ENS development is a complex process that requires coordinated migration, proliferation and differentiation of the involved cell types, directed outgrowth of neurites and the establishment of an interconnected neuronal and glial cell network $(15,16)$. Importantly, ENS development continues in the early postnatal period $(17,18)$ during which it is shaped by amongst others immune cells, microbiota and enteral nutrition (17).

Recently, we have shown in a preclinical ovine model that chronic chorioamnionitis is associated with significant structural ENS abnormalities (19). Importantly, these alterations corresponded with those found in infants with NEC, indicating that ENS changes following chorioamnionitis may predispose to later NEC development (19). Since inflammation is a dynamic process and the vulnerability of the fetus to injurious exposure during intra-uterine development varies, ENS alterations in response to inflammation can be time-dependent. As chorioamnionitis is often clinically silent and infants born after chorioamnionitis have been exposed to varying durations of intra-uterine inflammation, understanding time-dependent effects of intra-uterine inflammation on the ENS is clinically important to define optimal therapeutic strategies. Therefore, the aim of this study was to evaluate the time-dependent effects of 5 hour to 15 days of intra-uterine LPS exposure before premature delivery, on the intestinal submucosa, mucosa and ENS in fetal sheep. 


\section{Materials and Methods}

\section{Animal model and experimental procedures}

The experiments were approved by the animal ethics/care committee of the University of Western Australia (Perth, Australia; ethical approval number: RA/3/100/928).

The ovine model and experimental procedures were previously described $(6,20)$. In brief, 52 time-mated merino ewes carrying singleton fetuses were randomly assigned to eight different groups of six to seven animals. The pregnant ewes were IA injected under ultrasound guidance with $10 \mathrm{mg}$ Escherichia coli-derived LPS (O55:B5; Sigma-Aldrich, St. Louis, MO, USA) dissolved in saline at 5, 12, or 24 hours, or 2, 4, 8 or 15 days before preterm delivery at 125 days of gestation (equivalent of 30-32 weeks of human gestation for the gut; term gestation in sheep around 150 days). The study design is based on the clinically relevant situation that the gestational age of the infant is known, but not the length of exposure to inflammation. Hence, all samples were collected at the same gestational age and inflammation was induced at various times before sampling. Of importance, with a half-life time of 1.7 days, LPS persists in AF and can still be detected at 15 days (21). A group receiving IA injections of saline at variable gestational ages comparable to LPS injections, ranging from 5 hours to 15 days before preterm delivery, served as the controls (figure 1).

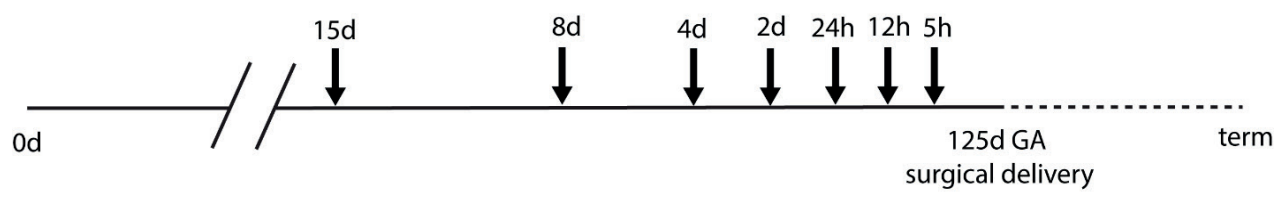

Figure 1. Study design. Pregnant ewes received an IA injection with $10 \mathrm{mg}$ LPS at 5,12 , or $24 \mathrm{~h}$ or $2,4,8$ or 15 days (black arrows) before preterm delivery at 122 days of gestation (term $\sim 150$ days). Control animals received an IA saline injection at comparable time points to LPS injections. Timing shown in gestational days.

Fetuses were delivered by cesarean section at 125 days of gestation and immediately euthanized with intravenous pentobarbitone $(100 \mathrm{mg} / \mathrm{kg})$. Fetuses of both sexes were used. At necropsy, the terminal ileum was sampled and fixed in $10 \%$ formalin or snap frozen. Formalin-fixed tissues were subsequently embedded in paraffin.

\section{Antibodies}

For immunohistochemistry, the following antibodies were used: polyclonal rabbit antimyeloperoxidase ([MPO]; A0398, Dakocytomation, Glostrup, Denmark) for identification of neutrophils, polyclonal rabbit anti-bovine protein gene product 9.5 ([PGP9.5]; Z5116, Dakocytomation) for the detection of enteric neurons, polyclonal rabbit anti-doublecortin (Ab18723, Abcam, Cambridge, UK) for the detection of immature neurons, polyclonal rabbit anti-glial fibrillary acidic protein ([GFAP]; Zo334, Dakocytomation) for identification of activated enteric glial cells and polyclonal rabbit anti-S100ß (PA5-16257, Invitrogen, Carlsbad, CA, USA) which is considered a general marker of enteric glial cells. 
The following secondary antibodies were used: peroxidase-conjugated polyclonal goat anti-rabbit (111-035-045, Jackson, WestGrove, PA, USA) (MPO), peroxidase-conjugated polyclonal swine anti-rabbit (P0399, DakoCytomation) (doublecortin) and BrightVision+ Poly-HRP-Anti Mouse/Rabbit IgG Biotin-free (ImmunoLogic, Duiven, the Netherlands) (PGP9.5), and biotin conjugated polyclonal swine anti-rabbit (E0353, DakoCytomation) (GFAP, S100ß).

\section{Immunohistochemistry}

Paraffin embedded formalin-fixed terminal ileum was cut into $4 \mu \mathrm{m}$ sections. Following deparaffinization and rehydration, sections were incubated in $0.3 \% \mathrm{H} 2 \mathrm{O} 2$ diluted in phosphorylated buffer saline ([PBS]; $\mathrm{pH} 7.4$ ) to block endogenous peroxidase activity. For PGP9.5, doublecortin and $\mathrm{S} 100 \beta$, antigen retrieval was achieved with citrate buffer. Nonspecific binding was blocked for 30 minutes at room temperature with $10 \%$ normal goat serum (NGS) in PBS (MPO), 5\% NGS in PBS (doublecortin), or $5 \%$ bovine serum albumin (BSA) in PBS (GFAP and S100ß) or for 10 minutes at room temperature with $20 \%$ fetal calf serum (FCS) in PBS (PGP9.5). Subsequently, sections were incubated with the primary antibody of interest for one hour (MPO) or overnight (others) followed by the secondary antibody for 30 minutes (MPO) or one hour (others). MPO, PGP9.5 and doublecortin were recognized using a peroxidase-conjugated secondary antibody; antibodies against GFAP and S100 $\beta$ were detected with avidin-biotin complex (Vectastain Elite ABC kit, Bioconnect, Huissen, the Netherlands). Substrate staining was performed with 3-amino-9ethylcarbazole ([AEC]; Merck, Darmstadt, Germany) (MPO), nickel-DAB (GFAP) or DAB (PGP9.5, doublecortin and S100 $\beta$ ). Hematoxylin (MPO, PGP9.5, doublecortin and S100 $\beta$ ) or nuclear fast red (GFAP) were used as nuclear counterstains.

\section{Quantification of immunohistochemical stainings}

The Ventana iScan HT slide scanner (Ventana Medical Systems, Oro Valley, AZ, USA) was used to scan stained tissue sections. With the use of Pannoramic Viewer (version 1.15.4, 3DHISTECH, Budapest, Hungary), an overview picture of the transverse section of the ileum was taken. Two investigators blinded to the experimental groups counted the number of mucosal MPO-positive cells. Leica QWin Pro (version 3.4.0, Leica Microsystems, Mannheim, Germany) was used to calculate the mucosal surface area. The average number of mucosal MPO-positive cells corrected for total mucosal tissue surface area is reported as MPO-positive cells per area per animal. Secondly, random images of the submucosal layer were taken (200x). In five non-overlapping high power fields, the number of submucosal MPO-positive cells was counted by two investigators blinded to the experimental groups. The average number of submucosal MPO-positive cells per animal of the five power fields is reported as MPO-positive cells per area. For PGP9.5, doublecortin, GFAP and S100ß, the surface of positively stained areas in the submucosal and myenteric ganglia and total surface area of the muscle layer were measured (Leica QWin Pro version 3.4.0, Leica Microsystems, Mannheim, Germany) in five non-overlapping high-power fields. The area fraction was calculated by dividing the positively stained surface area by the total surface area of the muscle layer. The average area fraction of the five high-power fields per animal is given as fold increase over the control value. The control value will be stated at one. All area fraction measurements were performed by one investigator blinded to the study groups. 


\section{RNA extraction and real-time PCR}

TRI reagent (Invitrogen)/chloroform extraction was used to extract RNA from snap frozen terminal ileum. Afterwards RNA was reverse transcribed into CDNA using sensifast CDNA Synthese kit (Bioline, London, UK). Quantitative real-time PCR (qPCR) was performed with the specific primers in Sensimix SYBR \& Fluorescein Kit (Bioline) using a 384-wells qPCR plate. qPCR reactions were performed in a LightCycler 480 Instrument (Roche Applied Science, Basel, Switzerland) for 45 cycles. Gene expression levels of tumor necrosis factor alpha (TNF-a), IL-8 and IL-10 were determined to assess terminal ileum inflammation. mRNA expression levels of neuronal nitric oxide synthase (nNOS) and choline acetyltransferase (CHAT) were determined to assess ENS motility signaling function. LinRegPCR software (version 2016.0, Heart Failure Research Center, Academic Medical Center, Amsterdam, the Netherlands) was used for qPCR data processing. The geometric mean of the expression levels of three reference genes (ribosomal protein S15 (RPS15), glyceraldehyde 3-phosphate dehydrogenase (GAPDH) and peptidylprolyl isomerase A (PPIA)) were calculated and used as a normalization factor. Data are expressed as fold increase over the control value. Sequences of the primers used are shown in Table 1.

Table 1. Primer sequences

\begin{tabular}{|c|c|c|}
\hline Primer & Forward & Reverse \\
\hline RPS15 & 5'-CGAGATGGTGGGCAGCAT-3' & 5'-GCTTGATTTCCACCTGGTTGA-3' \\
\hline GAPDH & 5'-GGAAGCTCACTGGCATGGC-3' & 5'-CCTGCTTCACCACCTTCTTG-3' \\
\hline PPIA & 5'-TTATAAAGGTTCCTGCTTTCACAGAA-3' & 5'-ATGGACTTGCCACCAGTACCA-3' \\
\hline IL-8 & 5'-GTTCCAAGCTGGCTGTTGCT-3' & 5'-GTGGAAAGGTGTGGAATGTGTTT-3' \\
\hline IL-10 & 5'-CATGGGCCTGACATCAAGGA-3' & 5'-CGGAGGGTCTTCAGCTTCTC-3' \\
\hline TNF-a & 5'-GCCGGAATACCTGGACTATGC-3' & 5'-CAGGGCGATGATCCCAAAGTAG-3' \\
\hline nNOS & 5'-CGGCTTTGGGGGTTATCAGT-3' & 5'-TTGCCCCATTTCCACTCCTC-3' \\
\hline CHAT & 5'-CCGCTGGTATGACAAGTCCC-3' & 5'-GCTGGTCTTCACCATGTGCT-3' \\
\hline
\end{tabular}

\section{Data analysis}

Statistical analyses were performed using GraphPad Prism (version 6.01, GraphPad Software Inc., La Jolla, CA, USA). Data are presented as median with interquartile range. Differences between the groups and the controls were analyzed using a nonparametric Kruskal-Wallis test followed by Dunn's post hoc test. Differences are considered statistically significant at $p \leq 0.05$. Differences with a $p<0.10$ are also taken into account because of the small study groups and because of potential biological relevance, and described as tendencies as previously described (22). This assumption will decrease the chance of a type II error, but increases the chance of a type I error. 


\section{Results}

\section{Chorioamnionitis induced intestinal inflammation}

A statistically significant increase in MPO-positive cells was seen in the mucosa 4 and 8 days after IA LPS exposure, compared to control ( $p<0.05$; table 2$)$.

Table 2. Immune cells count in the mucosal layer

\begin{tabular}{lcccccccc}
\hline & $\begin{array}{c}\text { Control } \\
(\mathbf{n}=\mathbf{6})\end{array}$ & $\begin{array}{c}\mathbf{5 h} \text { LPS } \\
(\mathbf{n}=\mathbf{6})\end{array}$ & $\begin{array}{c}\mathbf{1 2 h} \text { LPS } \\
(\mathbf{n}=\mathbf{7})\end{array}$ & $\begin{array}{c}\mathbf{2 4 h} \text { LPS } \\
(\mathbf{n}=\mathbf{7})\end{array}$ & $\begin{array}{c}\mathbf{2 d} \text { LPS } \\
(\mathbf{n}=\mathbf{6})\end{array}$ & $\begin{array}{c}\mathbf{4 d} \text { LPS } \\
(\mathbf{n}=\mathbf{6})\end{array}$ & $\begin{array}{c}\mathbf{8 d} \text { LPS } \\
(\mathbf{n}=\mathbf{7})\end{array}$ & $\begin{array}{c}\mathbf{1 5 d} \text { LPS } \\
(\mathbf{n}=\mathbf{6})\end{array}$ \\
\hline $\mathrm{MPO}+$ cell count & 102 & 74 & 159 & 76 & 151 & $\mathbf{3 5 4}^{*}$ & $\mathbf{3 3 2 *}$ & 224 \\
$\mathrm{SD}( \pm)$ & 110 & 77 & 166 & 51 & 105 & $\mathbf{1 6 2}$ & $\mathbf{1 0 1}$ & 96 \\
\hline
\end{tabular}

Values are expressed as median numbers of cells per square millimeter. SD: Standard deviation. KruskalWallis test with Dunn's post hoc test was performed. * $p<0.05$ compared to control.

In the submucosa, there was an increase of MPO-positive cells in animals exposed to 4 days of IA LPS, and submucosal MPO-positive cells still tended to be increased after 8 days of IA LPS exposure, compared to control ( $p<0.05$ and $p=0.08$; figure 2 ).

A

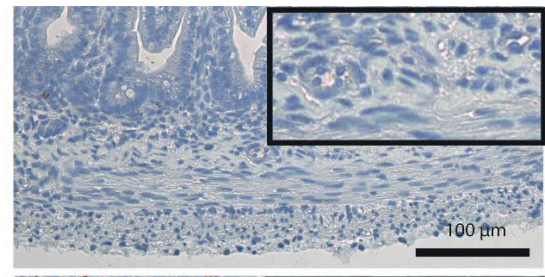

B

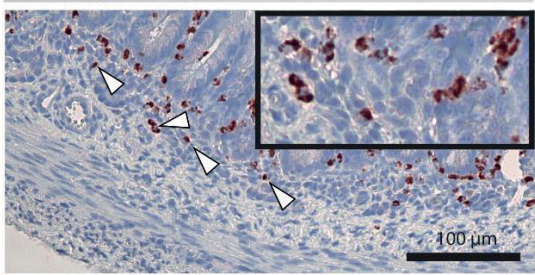

C

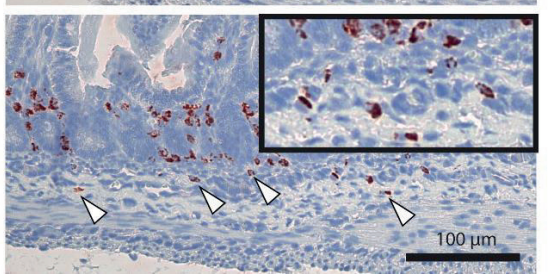

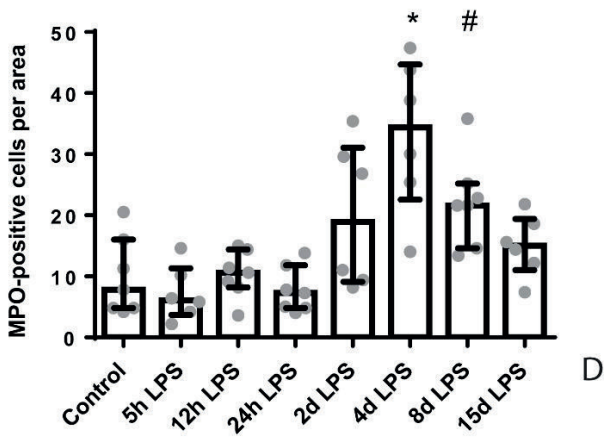

D

Figure 2. Representative images of submucosal neutrophil influx reflected by MPO-positive cell (indicated by white triangles) counts of the control (A), 4 days of IA LPS (B) and 8 days of IA LPS group (C). D: Increased MPO count in animals exposed to 4 and 8 days of IA LPS. ${ }^{*} p<0.01$ compared to control. $\# p=0.08$ compared to control.

Examination of underlying cytokine levels revealed increased ileal IL-8 mRNA levels after 24 hours and 4 days of IA LPS exposure, compared to control (both $p<0.05$; figure 3 ). 


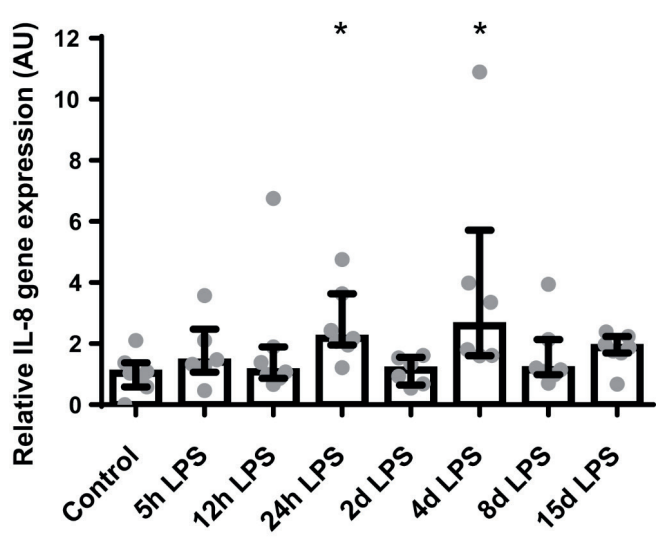

Figure 3. Relative gene expression of IL-8 in arbitrary unit (AU). Increased IL-8 gene expression in animals exposed to 24 hours and 4 days of IA LPS. ${ }^{*} p<0.05$ compared to control.

No differences were seen in IL-10 and TNF-a mRNA levels, compared to control (data not shown).

\section{Chorioamnionitis induced enteric nervous system alterations}

The PGP9.5-positive and doublecortin-positive surface areas in the submucosal plexus were unchanged in all groups compared to control (data not shown). In the myenteric plexus, the PGP9.5-positive surface area was decreased after 4 days of IA LPS exposure, compared to control ( $p<0.05$; figure 4$)$.

A

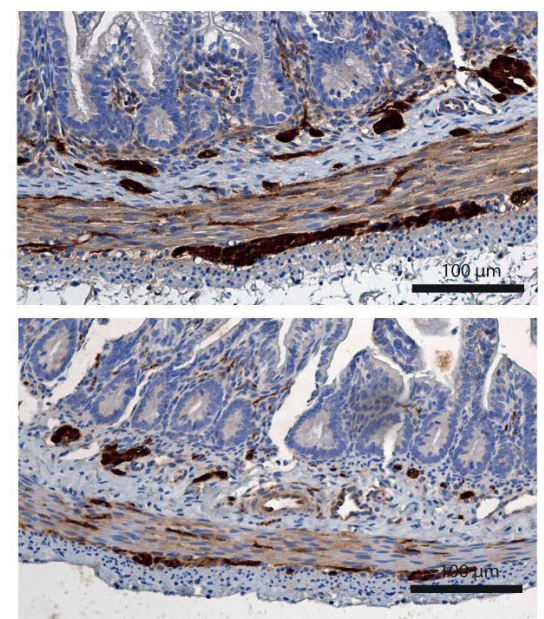

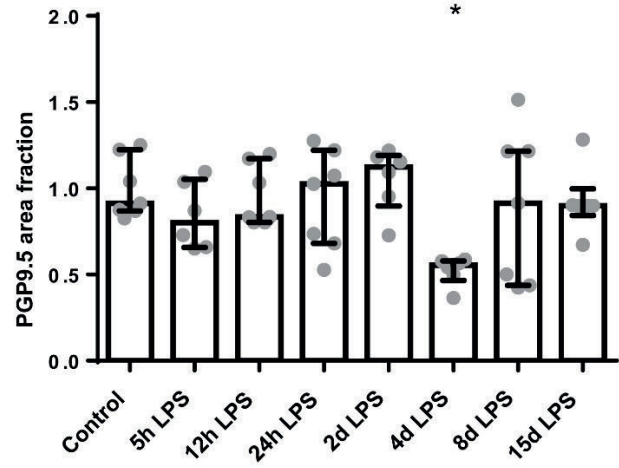

C

Figure 4. Representative images of PGP9.5 immunoreactivity in the submucosal and myenteric plexus of the control (A) and 4 days IA LPS group (B). Area fraction of PGP9.5 in the myenteric plexus (C) as fold increase over the control value. C: PGP9.5-positive surface area was decreased in animals exposed to 4 days of IA LPS. * $p<0.05$ compared to control. 
This reduction was resolved after 8 days of IA LPS exposure. At this time point, the doublecortin-positive surface area tended to be decreased in the myenteric plexus of LPS exposed animals, compared to control ( $p=0.07$; figure 5).

A

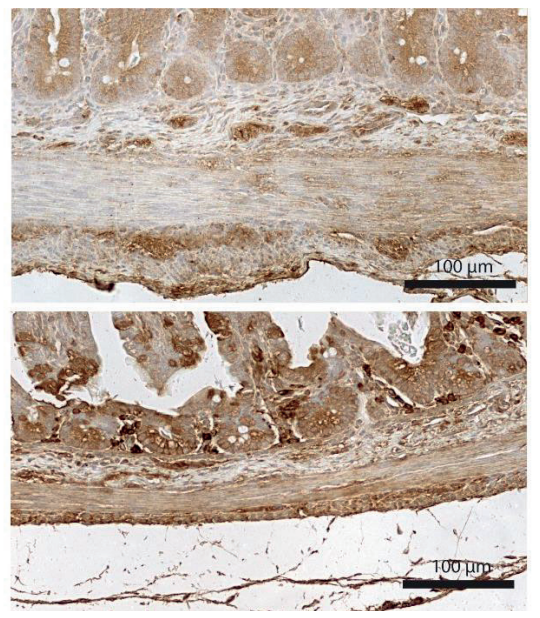

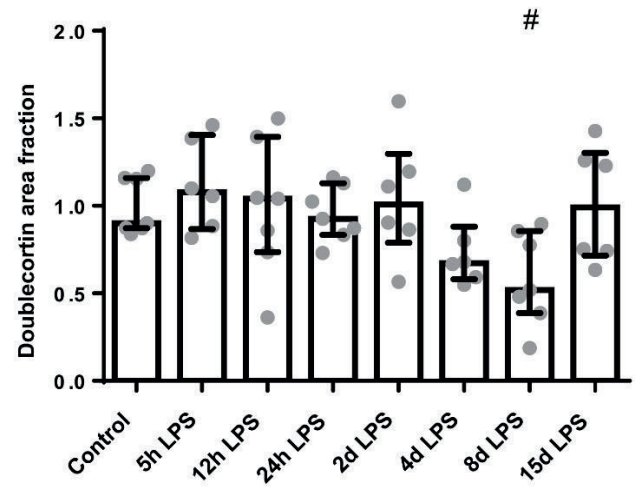

C

Figure 5. Representative images of doublecortin immunoreactivity in the submucosal and myenteric plexus of the control (A) and 8 days of IA LPS group (B). Area fraction of doublecortin in the myenteric plexus (C) as fold increase over the control value. C: Doublecortin-positive surface area tended to be decreased in animals exposed to 8 days of IA LPS. \# $\mathrm{p}=0.07$ compared to control.

In the submucosal plexus, no differences in the GFAP-positive surface areas were observed (data not shown), while in the myenteric plexus, the GFAP-positive surface area was increased in animals exposed to 2 days of IA LPS, compared to control ( $p<0.05$; figure 6$)$.

A

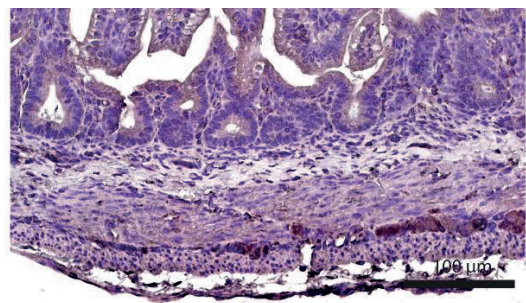

B

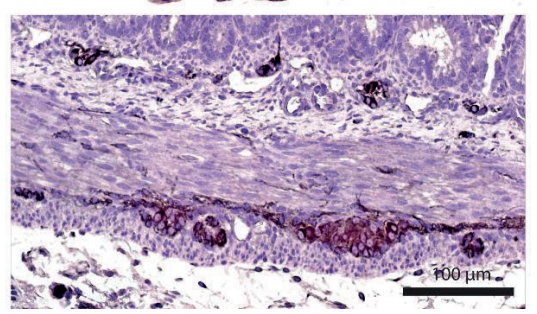

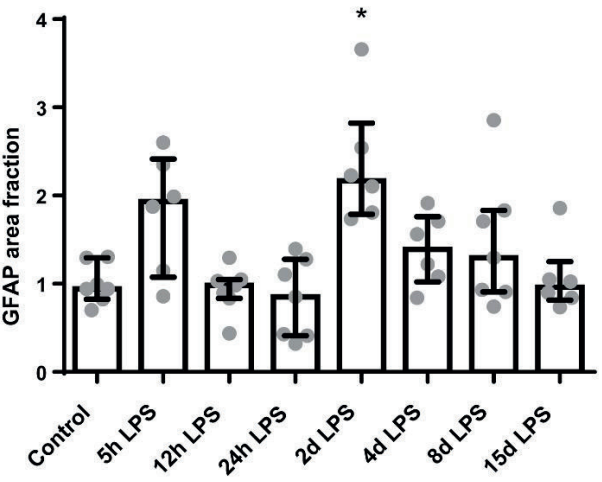

C

Figure 6. Representative images of GFAP immunoreactivity in the submucosal and myenteric plexus of the control (A) and 2 days of IA LPS group (B). Area fraction of GFAP in the myenteric plexus (C) as fold increase over the control value. C: GFAP-positive surface area in the myenteric plexus was increased in animals exposed to 2 days of IA LPS. ${ }^{*} p<0.05$ compared to control. 
The S100 $\beta$-positive surface area in the submucosal plexus tended to be decreased in animals exposed to 8 days of IA LPS, compared to control ( $p=0.09$; figure 7$)$. In the myenteric plexus, the $S 100 \beta$-positive surface area was decreased in animals exposed to 4 days of IA LPS, compared to control ( $p<0.05$; figure 7).

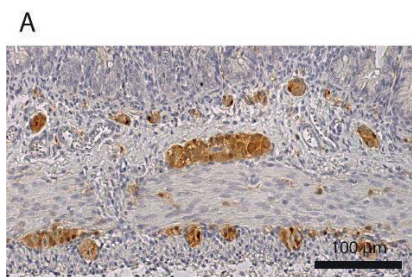

B

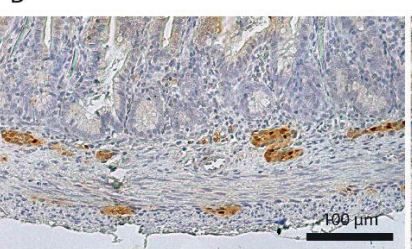

C

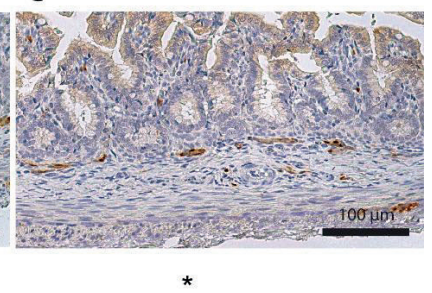

D
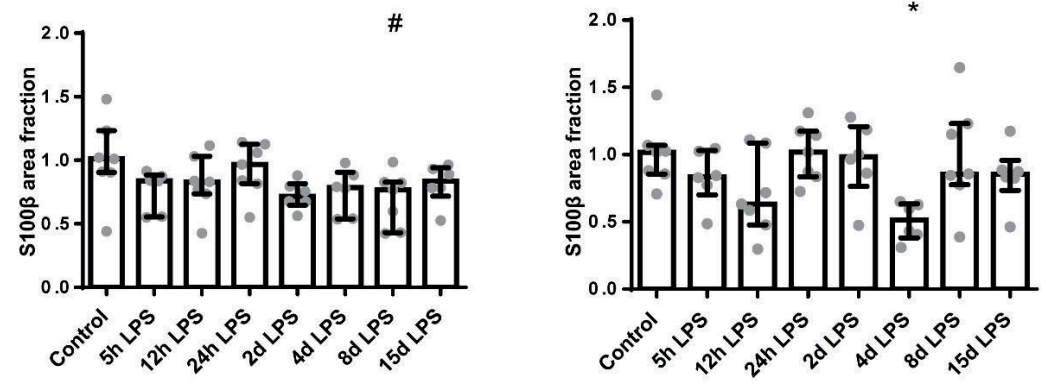

E

Figure 7. Representative images of $S 100 \beta$ immunoreactivity in the submucosal and myenteric plexus of the control (A), 4 days of IA LPS (B) and 8 days of IA LPS group (C). Area fraction of S100 $\beta$ in the submucosal (D) and myenteric plexus (E) as fold increase over the control value. D: S100ß-positive surface area tended to be decreased in animals exposed to 8 days of IA LPS. \# $p=0.09$ compared to control. E: S100 $\beta$-positive surface area is decreased in animals exposed to 4 days of IA LPS. ${ }^{*} p<0.05$ compared to control.

No differences in nNOS and CHAT mRNA expression were observed between the groups (data not shown). 


\section{Discussion}

In the current study, mucosal and submucosal intestinal inflammation was observed in the terminal ileum after 4 days of IA LPS exposure. On mRNA level, gut inflammation (IL-8) also occurs after 24 hours of IA LPS exposure, and this time point overlaps with the fetal systemic immune response, characterized by increased circulatory IL-6 levels (6). In utero gastro-intestinal transit studies showed it takes approximately 24 hours for the swallowed $\mathrm{AF}$ to reach the mid-ileum (unpublished findings). Hence, this early inflammatory response in the terminal ileum is probably not caused by a local process, but solely the result of fetal systemic inflammation. In line, previous research in the same ovine model has shown that chorioamnionitis induced gut inflammation is the combined effect of direct gut exposure to LPS and a lung-mediated systemic inflammatory response (5). It is possible that the early intestinal IL-8 peak contributes to the submucosal and mucosal increase of inflammatory cells at 4 days of IA LPS exposure through stimulation of chemotaxis (23).

Interestingly, the most evident signs of ENS alterations were also seen after 4 days, and after 8 days of IA LPS exposure. After 4 days IA LPS exposure, the myenteric plexus PGP9.5positive surface area was decreased, indicating a loss of enteric neurons and/or reduction of PGP9.5 immunoreactivity of enteric nerve cells. Since the doublecortin-positive (immature neurons) surface area remained unchanged, this was probably the result of affected mature neurons. The reduced PGP9.5-positive surface area after 4 days of IA LPS exposure was recovered after 8 days of IA LPS exposure. The doublecortin-positive surface area tended to decrease at this time point. These findings might indicate that an initial loss of mature neurons is compensated by an accelerated maturation of immature neurons. Whether such an accelerated maturation is sufficient to fully compensate for the identified loss of neurons remains to be elucidated. These findings combined with the unaltered PGP9.5-positive and doublecortin-positive surface area after 2 and 7 days of IA LPS exposure in a previous study (19), show that the ENS changes found are time-dependent and may recover following prolonged intra-uterine inflammation. Interestingly, in a previous study, a similar loss of mature neurons was observed after chronic IA exposure to UP, indicating that different inflammatory triggers can induce similar ENS damage (19).

Enteric glial cells are important for neuronal maintenance, survival, and function (24), and are capable of generating enteric neurons in response to injury $(25,26)$. In addition, enteric glia respond, in a manner similar as reactive astrogliosis in the central nervous system, to ENS injury and inflammation by changing both their morphology and their expression of key proteins such as GFAP $(27,28)$. The neuronal loss in the myenteric plexus after four days of LPS exposure is accompanied with a reduced S100 $\beta$-positive surface area, likely representing a loss of glial cells and/or loss of $S 100 \beta$ immunoreactivity within glial cells, as was earlier described during chronic IA UP exposure (19). Interestingly, this loss of neurons and glial cells is preceded by an increased myenteric plexus GFAP immunoreactivity after 2 days of LPS exposure. It is likely that the observed glial cell response results from fetal systemic inflammation and/or intestinal inflammation, since pro-inflammatory cytokines have been shown to induce GFAP expression in enteric glial cells (29). Moreover, as activated enteric glial cells can secrete various cytokines and other mediators involved in the infiltration and activation of immune cells $(30,31)$, the observed glial cell reaction can 
contribute to the intestinal influx of neutrophils observed after 4 days of IA LPS exposure. Since a glial cell response in the context of intestinal inflammation can be destructive (32) and eventually neuroregenerative (33), it is to date unclear whether it contributes to the loss of neurons and glial cells, or is a protective mechanism that falls short with prolonged inflammation.

In this study, the most profound ENS changes were found in the myenteric plexus, rather than the submucosal plexus. This is in concordance with earlier findings in fetal lambs that were chronically IA exposed to UP (19). Moreover, inflammation driven pathological changes of the ENS are more often found in the myenteric plexus than in the submucosal plexus (34). The mechanisms behind this apparent increased vulnerability of the myenteric plexus remain to be elucidated. At present, we can only speculate about the mechanisms responsible for the observed differences because multiple possible explanations are in play. First, since the ENS undergoes rapid structural growth in utero, the composition of the submucosal and myenteric plexus might be differently altered by the combination of ongoing developmental processes and LPS exposure. Alternatively, the migratory pattern of cells in these plexi might be different during this essential developmental period of the ENS. Second, the macrophages in the plexus, which are in close proximity to neuronal cell bodies and nerve fibers, undergo differentiation towards a multitude of subsets depending on microenvironment but also depending on developmental stage and bacterial colonization. Our findings indicate that these cells play a role in the differential response of the submucosal and myenteric plexus, although the reason for that remains speculative. Notably, the transcriptional profiles of macrophages gradually differ from the lumen to the myenteric plexus. As a result, the macrophages closer to the lumen play an important role by sampling luminal bacteria and initiating adaptive immune responses to clear pathogenic bacteria, whereas macrophages in the muscularis, which are comparatively more distant from luminal stimulation, are primarily involved in tissue protection and regulation of the activity of enteric neurons and peristalsis $(35,36)$. It is tempting to speculate that phenotypical differences of these immune cells following exposure to a bacterial stimulus in the different plexi are involved in the observed differences between the submucosal and myenteric plexus.

At present, it is unclear whether the observed changes have postnatal functional consequences. As the mRNA expression of CHAT and nNOS are unchanged, in utero motility signaling function could be unaltered. This confirms and extends previous findings in fetal lambs chronically IA exposed to UP (19). The resolved inflammation and the recovery of (immature) neurons and glial cells after fifteen days of IA LPS exposure indicate that damage due to IA LPS exposure probably can be repaired in utero. Nevertheless, it is likely that a child that is born prematurely with ongoing inflammation due to FIRS will experience additional postnatal inflammatory stimuli such as mechanical ventilation (37) or sepsis (38). The effects of these postnatal exposures on the ENS should be studied in order to shed light on the long term consequences of (intra-uterine) inflammation for ENS development and function. Notably, 4 to 8 days after the start of intra-uterine infection could very well be the window of vulnerability in which additional inflammation may have a higher impact as the ENS is already affected at this time point. 
A limitation of this study is the relatively low number of animals per group, which is an unavoidable shortcoming of the translational large animal model. Secondly, the current set-up with the fixed moment of premature birth does not exclude a potential influence of gestational age at start of intra-uterine infection. Thirdly, in the current study we were unable to unravel the mechanisms behind the observed changes, as no serial sampling was applied following a specific injection time point.

In summary, submucosal intestinal inflammation was detected after 4 days of IA LPS exposure that coincided with gut mucosal and fetal systemic inflammation. At the same time point, a loss of PGP9.5 and S100 $\beta$ immunoreactivity in the myenteric plexus was observed. These changes are preceded by a glial cell response with systemic inflammation and local gut inflammation. Although initial ENS damage seemed to recover after prolonged IA LPS exposure, additional postnatal inflammatory hits that a premature born child is likely to encounter may further harm the ENS and influence functional outcomes. In this context, 4 to 8 days after the start of an intra-uterine infection may be a window of increased ENS vulnerability, indicating that therapeutic interventions should ideally start before or at this time point. 


\section{References}

1. Galinsky R, Polglase GR, Hooper SB, Black MJ, Moss TJ. The consequences of chorioamnionitis: preterm birth and effects on development. J Pregnancy. 2013;2013:412831 DOI: 10.1155/2013/412831.

2. Goldenberg RL, Hauth JC, Andrews WW. Intrauterine infection and preterm delivery. N Engl J Med. 2000;342(20):1500-7 DOI: 10.1056/NEJM200005183422007.

3. Kim CJ, Romero R, Chaemsaithong P, Chaiyasit N, Yoon BH, Kim YM. Acute chorioamnionitis and funisitis: definition, pathologic features, and clinical significance. Am J Obstet Gynecol. 2015;213(4 Suppl):S29-52 DOI: 10.1016/j.ajog.2015.08.040.

4. Gantert M, Been JV, Gavilanes AW, Garnier Y, Zimmermann LJ, Kramer BW. Chorioamnionitis: a multiorgan disease of the fetus? J Perinatol. 2010;30 Suppl:S21-30 DOI: 10.1038/jp.2010.96.

5. Wolfs TG, Kramer BW, Thuijls G, Kemp MW, Saito M, Willems MG, Senthamarai-Kannan P, Newnham JP, Jobe AH, Kallapur SG. Chorioamnionitis-induced fetal gut injury is mediated by direct gut exposure of inflammatory mediators or by lung inflammation. Am J Physiol Gastrointest Liver Physiol. 2014;306(5):G382-93 DOI: 10.1152/ajpgi.00260.2013.

6. Gussenhoven R, Westerlaken RJJ, Ophelders D, Jobe AH, Kemp MW, Kallapur SG, Zimmermann LJ, Sangild PT, Pankratova S, Gressens P, Kramer BW, Fleiss B, Wolfs T. Chorioamnionitis, neuroinflammation, and injury: timing is key in the preterm ovine fetus. J Neuroinflammation. 2018;15(1):113 DOI: 10.1186/s12974-018-1149-x.

7. Been JV, Lievense S, Zimmermann LJ, Kramer BW, Wolfs TG. Chorioamnionitis as a risk factor for necrotizing enterocolitis: a systematic review and meta-analysis. J Pediatr. 2013;162(2):236-42 e2 DOI: 10.1016/j.jpeds.2012.07.012.

8. Neu J, Walker WA. Necrotizing enterocolitis. N Engl J Med. 2011;364(3):255-64 DOI: 10.1056/ NEJMra1005408.

9. Neu J, Pammi M. Necrotizing enterocolitis: The intestinal microbiome, metabolome and inflammatory mediators. Semin Fetal Neonatal Med. 2018 DOI: 10.1016/j.siny.2018.08.001.

10. Sigge W, Wedel T, Kuhnel W, Krammer HJ. Morphologic alterations of the enteric nervous system and deficiency of non-adrenergic non-cholinergic inhibitory innervation in neonatal necrotizing enterocolitis. Eur J Pediatr Surg. 1998;8(2):87-94 DOI: 10.1055/s-2008-1071128.

11. Wedel T, Krammer HJ, Kuhnel W, Sigge W. Alterations of the enteric nervous system in neonatal necrotizing enterocolitis revealed by whole-mount immunohistochemistry. Pediatr Pathol Lab Med. 1998;18(1):57-70.

12. Fagbemi AO, Torrente F, Puleston J, Lakhoo K, James S, Murch SH. Enteric neural disruption in necrotizing enterocolitis occurs in association with myenteric glial cell CCL20 expression. J Pediatr Gastroenterol Nutr. 2013;57(6):788-93 DOI: 10.1097/MPG.0b013e3182a86fd4.

13. Zhou Y, Yang J, Watkins DJ, Boomer LA, Matthews MA, Su Y, Besner GE. Enteric nervous system abnormalities are present in human necrotizing enterocolitis: potential neurotransplantation therapy. Stem Cell Res Ther. 2013;4(6):157- DOI: 10.1186/scrt387.

14. Furness JB. The enteric nervous system and neurogastroenterology. Nat Rev Gastroenterol Hepatol. 2012;9(5):286-94 DOI: 10.1038/nrgastro.2012.32.

15. Rao M, Gershon MD. Enteric nervous system development: what could possibly go wrong? Nat Rev Neurosci. 2018;19(9):552-65 DOI: 10.1038/s41583-018-0041-0.

16. Lake J,Heuckeroth RO. Entericnervous system development:migration, differentiation, and disease. Am J Physiol Gastrointest Liver Physiol. 2013;305(1):G1-24 DOI: 10.1152/ajpgi.00452.2012. 
17. Hao MM, Foong JP, Bornstein JC, Li ZL, Vanden Berghe P, Boesmans W. Enteric nervous system assembly: Functional integration within the developing gut. Dev Biol. 2016;417(2):168-81 DOI: 10.1016/j.ydbio.2016.05.030.

18. Burns AJ, Roberts RR, Bornstein JC, Young HM. Development of the enteric nervous system and its role in intestinal motility during fetal and early postnatal stages. Semin Pediatr Surg. 2009;18(4):196-205 DOI: 10.1053/j.sempedsurg.2009.07.001.

19. Heymans $C$, de Lange IH, Hutten MC, Lenaerts $K$, de Ruijter NJE, Kessels L, Rademakers $G$, Melotte V, Boesmans W, Saito M, Usuda H, Stock SJ, Spiller OB, Beeton ML, Payne MS, Kramer BW, Newnham JP, Jobe AH, Kemp MW, van Gemert WG, Wolfs T. Chronic Intra-Uterine Ureaplasma parvum Infection Induces Injury of the Enteric Nervous System in Ovine Fetuses. Front Immunol. 2020;11:189 DOI: 10.3389/fimmu.2020.00189.

20. Kuypers E, Wolfs TG, Collins JJ, Jellema RK, Newnham JP, Kemp MW, Kallapur SG, Jobe AH, Kramer BW. Intraamniotic lipopolysaccharide exposure changes cell populations and structure of the ovine fetal thymus. Reprod Sci. 2013;20(8):946-56 DOI: 10.1177/1933719112472742.

21. Newnham JP, Kallapur SG, Kramer BW, Moss TJ, Nitsos I, Ikegami M, Jobe AH. Betamethasone effects on chorioamnionitis induced by intra-amniotic endotoxin in sheep. Am J Obstet Gynecol. 2003;189(5):1458-66 DOI: 10.1067/s0002-9378(03)00758-0.

22. Willems MG, Ophelders DR, Nikiforou M, Jellema RK, Butz A, Delhaas T, Kramer BW, Wolfs TG. Systemic interleukin-2 administration improves lung function and modulates chorioamnionitisinduced pulmonary inflammation in the ovine fetus. Am J Physiol Lung Cell Mol Physiol. 2016;310(1):L1-7 DOI: 10.1152/ajplung.00289.2015.

23. Russo RC, Garcia CC, Teixeira MM, Amaral FA. The CXCL8/IL-8 chemokine family and its receptors in inflammatory diseases. Expert Rev Clin Immunol. 2014;10(5):593-619 DOI: 10.1586/1744666x.2014.894886.

24. De Giorgio R, Giancola F, Boschetti E, Abdo H, Lardeux B, Neunlist M. Enteric glia and neuroprotection: basic and clinical aspects. Am J Physiol Gastrointest Liver Physiol. 2012;303(8):G887-93 DOI: 10.1152/ajpgi.00096.2012.

25. Joseph NM, He S, Quintana E, Kim YG, Nunez G, Morrison SJ. Enteric glia are multipotent in culture but primarily form glia in the adult rodent gut. J Clin Invest. 2011;121(9):3398-411 DOI: 10.1172/ JCI58186.

26. Laranjeira C, Sandgren K, Kessaris N, Richardson W, Potocnik A, Vanden Berghe P, Pachnis V. Glial cells in the mouse enteric nervous system can undergo neurogenesis in response to injury. J Clin Invest. 2011;121(9):3412-24 DOI: 10.1172/jci58200.

27. Boesmans W, Lasrado R, Vanden Berghe P, Pachnis V. Heterogeneity and phenotypic plasticity of glial cells in the mammalian enteric nervous system. Glia. 2015;63(2):229-41 DOI: 10.1002/ glia.22746.

28. Rosenbaum C, Schick MA, Wollborn J, Heider A, Scholz CJ, Cecil A, Niesler B, Hirrlinger J, Walles $\mathrm{H}$, Metzger M. Activation of Myenteric Glia during Acute Inflammation In Vitro and In Vivo. PLoS One. 2016;11(3):e0151335 DOI: 10.1371/journal.pone.0151335.

29. von Boyen GB, Steinkamp M, Reinshagen M, Schafer KH, Adler G, Kirsch J. Proinflammatory cytokines increase glial fibrillary acidic protein expression in enteric glia. Gut. 2004;53(2):222-8 DOI: 10.1136/gut.2003.012625.

30. Stoffels B, Hupa KJ, Snoek SA, van Bree S, Stein K, Schwandt T, VilzTO, Lysson M, Veer CV, Kummer MP, Hornung V, Kalff JC, de Jonge WJ, Wehner S. Postoperative ileus involves interleukin-1 receptor signaling in enteric glia. Gastroenterology. 2014;146(1):176-87.e1 DOI: 10.1053/j. gastro.2013.09.030. 
31. Sharkey KA. Emerging roles for enteric glia in gastrointestinal disorders. J Clin Invest. 2015;125(3):918-25 DOI: 10.1172/jci76303.

32. Brown IA, McClain JL, Watson RE, Patel BA, Gulbransen BD. Enteric glia mediate neuron death in colitis through purinergic pathways that require connexin-43 and nitric oxide. Cell Mol Gastroenterol Hepatol. 2016;2(1):77-91 DOI: 10.1016/j.jcmgh.2015.08.007.

33. Belkind-Gerson J, Graham HK, Reynolds J, Hotta R, Nagy N, Cheng L, Kamionek M, Shi HN, Aherne CM, Goldstein AM. Colitis promotes neuronal differentiation of Sox2+ and PLP1+ enteric cells. Sci Rep. 2017;7(1):2525 DOI: 10.1038/s41598-017-02890-y.

34. De Giorgio R, Guerrini S, Barbara G, Stanghellini V, De Ponti F, Corinaldesi R, Moses PL, Sharkey $\mathrm{KA}$, Mawe GM. Inflammatory neuropathies of the enteric nervous system. Gastroenterology. 2004;126(7):1872-83 DOI: 10.1053/j.gastro.2004.02.024.

35. Gabanyi I, Muller PA, Feighery L, Oliveira TY, Costa-Pinto FA, Mucida D. Neuro-immune Interactions Drive Tissue Programming in Intestinal Macrophages. Cell. 2016;164(3):378-91 DOI: 10.1016/j. cell.2015.12.023.

36. De Schepper S, Verheijden S, Aguilera-Lizarraga J, Viola MF, Boesmans W, Stakenborg N, Voytyuk I, Schmidt I, Boeckx B, Dierckx de Casterle I, Baekelandt V, Gonzalez Dominguez E, Mack M, Depoortere I, De Strooper B, Sprangers B, Himmelreich U, Soenen S, Guilliams M, Vanden Berghe P, Jones E, Lambrechts D, Boeckxstaens G. Self-Maintaining Gut Macrophages Are Essential for Intestinal Homeostasis. Cell. 2018;175(2):400-15 e13 DOI: 10.1016/j.cell.2018.07.048.

37. Bose $\mathrm{CL}$, Laughon MM, Allred EN, O'Shea TM, Van Marter LJ, Ehrenkranz RA, Fichorova RN, Leviton A, Investigators ES. Systemic inflammation associated with mechanical ventilation among extremely preterm infants. Cytokine. 2013;61(1):315-22 DOI: 10.1016/j.cyto.2012.10.014.

38. Machado JR, Soave DF, da Silva MV, de Menezes LB, Etchebehere RM, Monteiro ML, dos Reis MA, Correa RR, Celes MR. Neonatal sepsis and inflammatory mediators. Mediators Inflamm. 2014;2014:269681 DOI: 10.1155/2014/269681. 



\section{CHAPTER 4}




\section{Alterations of the enteric nervous system and intestinal motility in a mouse necrotizing enterocolitis model}

C. Heymans*, I.H. de Lange*, I.G. de Plaen, L.C.G.A. Kessels, B.W. Kramer, K. Lenaerts, W.G. van Gemert, T.G.A.M. Wolfs

* Contributed equally 


\section{Abstract}

\section{Background}

Necrotizing enterocolitis (NEC), characterized by severe intestinal inflammation and in later stages bowel necrosis, is one of the most common intestinal emergencies in premature neonates. Alterations of the enteric nervous system (ENS) and immature intestinal motility are associated with NEC. Although experimental NEC models are important for understanding NEC pathophysiology, the ENS remains essentially unstudied in models for NEC. To bridge this knowledge gap, we studied the ENS and intestinal transit in a verified mouse NEC model.

\section{Materials and Methods}

NEC was induced in newborn C57BL/6 mice by formula feeding, supplementation of oral commensal bacteria and LPS, asphyxia and cold stress. The ENS and intestinal transit were studied.

\section{Results}

In contrast to earlier rodent studies, mice in the experimental NEC group $(0-72 \mathrm{~h})$ had more mature neurons and glial cells and increased presence of presynaptic vesicles in the submucosal and myenteric plexus compared to controls. At $24 \mathrm{~h}$, intestinal transit was increased, whereas a decrease of the intestinal motility was detected at $48 \mathrm{~h}$.

\section{Conclusion}

The observed ENS changes are considered to represent a relative mild disease state of the clinical NEC spectrum, with an ENS response likely preceding glial cell and neuronal loss. 


\section{Introduction}

Necrotizing enterocolitis (NEC), characterized by severe intestinal inflammation and in later stages bowel necrosis, is one of the most common intestinal emergencies in premature neonates (1). It affects around 5 to $10 \%$ of very low birth weight infants and incidences are rising, which is primarily caused by improved early survival rates of premature newborns $(2,3)$. Mortality generally ranges from 15 to $30 \%$, but increases up to $50 \%$ for extremely low birth weight children treated surgically $(1,4,5)$. Infants that do survive are at increased risk for long term morbidities such as growth retardation $(6,7)$ and neurodevelopmental delay $(8,9)$.

The pathogenesis of NEC is multifactorial and still incompletely understood, however, immaturity of the intestinal immune system and abnormal bacterial colonization are clearly involved in the etiology of the excessive inflammation $(1,10)$. In addition, an immature gut motility is regarded as a risk factor for NEC development (10). In line, alterations of the enteric nervous system (ENS) are associated with NEC; in intestinal specimen from infants with NEC, structural ENS abnormalities including the loss of neurons and enteric glial cells have been found (11-13). In addition, reduced expression of neuronal nitric oxide synthase (nNOS) and choline acetyltransferase (CHAT), markers for motility signaling functions of the ENS, during acute NEC suggest a functional impairment of the ENS (14). Finally, evidence from a preterm pig NEC model, in which region-dependent changes in gut transit time preceded radiological signs of NEC, suggests dysmotility is involved in NEC pathophysiology (15).

The ENS autonomously regulates gastrointestinal activity, including secretion, absorption, and motility and contributes to gut integrity $(16,17)$. Furthermore, it is involved in the shaping of the mucosal immune system and the intestinal microbiome $(16,18)$. Development of the ENS requires a complex coordinated migration, proliferation and differentiation of neural crest progenitors, directed neurite growth, and establishment of a network of glial cells and interconnected neurons $(19,20)$. ENS development continues postnatally and in premature neonates the ENS is still immature (19).

As NEC research in infants is notably difficult for ethical reasons, animal models of NEC are frequently used $(21,22)$. Interestingly, ENS alterations in conjunction with objectified altered intestinal motility remain essentially unstudied in models for NEC. To bridge this knowledge gap, we studied the ENS and intestinal transit in a verified and standardized mouse NEC model (23). 


\section{Materials and Methods}

\section{Experimental design}

The animal studies were approved by the Children's Memorial Research Center (CMRC), Institutional Animal Care and Use Committee (Chicago, IL, USA) and the National Research Council's guide for the care and use of laboratory animals was followed. Male and female C57BL/6 mice from Jackson Laboratory (Bar Harbor, ME, USA) were housed in a barrier facility and time-mated pregnancies were induced overnight. After natural delivery, animals were randomly assigned to the two experimental groups: control and NEC. Mouse pups assigned to the NEC group were separated from the dams and placed in a humidified 37-degree incubator prior to start of the experiment. NEC was induced in neonatal mice as previously described (23). Briefly, at the start of the study period, 108 CFU of standardized commensal bacteria including $5 \mathrm{mg} / \mathrm{kg}$ LPS were administered by orogastric gavage within 12 hours after birth. Thereafter, enteral feeding by orogastric gavage was initiated and repeated every 3 hours. Pups were fed with Esbilac (PetAg, Hampshire, IL, USA) formula (33 $\mathrm{g}$ dissolved in $100 \mathrm{~mL}$ water), 30, 40 and $50 \mu \mathrm{L}$ respectively on day 1,2 and 3. In addition, pups were exposed to a short period of asphyxia ( $100 \%$ nitrogen for 60 seconds) followed by cold stress (4 degrees for 10 minutes) twice daily. Pups remaining with the dam and being dam fed during the entire study period served as controls. Mouse pups were continuously monitored for signs of distress (abdominal distension, lethargy, respiratory distress) and pups were euthanized by decapitation if severe distress was present. Those surviving were euthanized at the end of the 72-hour study period. Time of euthanasia was documented and a 72-hour survival curve was defined. After euthanasia, intestines were collected and fixed with $10 \%$ buffered formalin for histological analyses. Pups found dead were excluded from the analyses. The remaining 85 mice pups were used for the 72-hour survival and histological analyses (control: $n=28$ and NEC protocol: $n=57)$. In addition, 29 mice pups were used for analysis of intestinal transit (control: $n=13$ and NEC protocol: $\mathrm{n}=16$ ).

\section{Antibodies}

The following primary antibodies were used for immunohistochemistry: polyclonal rabbit anti-doublecortin (Ab18723, Abcam, Cambridge, UK) to detect immature neurons, monoclonal mouse anti-neuronal nuclear protein (NeuN) (clone A60, IHCR1001, Merck, Darmstadt, Germany) to identify mature neurons, polyclonal rabbit anti-S100 $\beta$ (PA516257, Thermofisher, Waltham, MA, USA) for the detection of glial cells and monoclonal rabbit anti-synaptophysin (Ab32127, Abcam, Cambridge, UK) for the identification of presynaptic vesicles.

Secondary antibodies used were: biotin-conjugated polyclonal donkey anti-rabbit (711065-152, Jackson, West Grove, PA, USA) (for detection of doublecortin), biotin conjugated polyclonal goat anti-mouse (E0433, DakoCytomation, Glostrup, Denmark) (for detection of NeuN) and biotin-conjugated polyclonal swine anti-rabbit (E0353 DakoCytomation, Glostrup, Denmark) (for detection of S100 $\beta$ and synaptophysin). 


\section{Histochemical staining}

Formalin-fixed intestine was embedded in paraffin and $4-\mu \mathrm{m}$ sections were cut. A Haematoxylin and Eosin (H\&E) staining was performed to determine the NEC severity stage as explained below.

\section{Immunohistochemistry}

After deparaffinization and rehydration of paraffin-embedded sections, endogenous peroxidase activity was blocked with $0.3 \% \mathrm{H} 2 \mathrm{O} 2$ diluted in phosphorylated buffer saline (PBS). Heat-mediated antigen retrieval was performed by boiling in $10 \mathrm{mM}$ sodium-citrate buffer ( $\mathrm{pH}$ 6.0). Non-specific binding was blocked for 30 minutes at room temperature with $5 \%$ bovine serum albumin (BSA) in PBS (doublecortin, NeuN and S100 3 ), or $5 \%$ normal goat serum (NGS) in PBS (synaptophysin). Thereafter, sections were incubated with the primary antibody of interest and subsequently incubated with the selected secondary antibody. The primary antibodies were visualized with an avidin-biotin complex (Vectastain Elite $A B C$ kit, Bio-connect, Huissen, the Netherlands). Immunoreactivity for doublecortin and S100 $\beta$ was detected by using diaminobenzidine (DAB). Immunoreactivity for NeuN and synaptophysin was detected by using nickel-3, 3'-DAB. Haematoxylin (doublecortin and $\mathrm{S} 100 \beta)$ or nuclear fast red (NeuN and synaptophysin) were used as counterstain.

\section{Histological analysis}

$\mathrm{H} \& \mathrm{E}$ slides were scored on severity of intestinal histological injury as described before (23). In short, H\&E slides were assessed by an independent investigator blinded to the experimental groups, to determine the intestinal damage score based on the area of worst injury. The following scoring system was used as previously described (23): Grade 0: intact villi; 1 : superficial epithelial cell sloughing; 2 : mid-villus necrosis; 3 : complete villus necrosis; 4: transmural necrosis. Mice with damage scores $\geq 2$ are considered to have NEC.

\section{Quantification of immunohistochemical stainings}

The stained tissue sections were scanned with the Ventana iScan HT slide scanner (Ventana Medical Systems, Oro Valley, AZ, USA). Of these images, viewed with Ventana Image Viewer (version 3.1.4, Ventana Medical Systems, Oro Valley, AZ, USA), random snapshots of cross sections of the small intestine were taken (200x).

Doublecortin (immature neurons), NeuN (mature neurons), S100 $\beta$ (glial cells) and synaptophysin (presynaptic vesicles) were studied as markers for the ENS. Doublecortin, NeuN, S100 $\beta$ and synaptophysin positively stained areas in the submucosal and myenteric ganglia were measured in five non-overlapping high power fields by calculating the area fraction (\%) with Leica QWin Pro (version 3.4.0, Leica Microsystems, Mannheim, Germany). The area fraction was calculated by dividing the positively stained areas in the ganglia of the submucosal or myenteric plexus by the total surface of the muscle layer. The average area fraction per high-power field per animal is given. The area fractions were measured by an investigator blinded to the study groups.

\section{Intestinal transit}

Mice included for the intestinal transit experiment were subjected to the same experimental set-up as described above. At 24 hours after subjection to the NEC protocol 
(control: $n=8$ and NEC: $n=9$ ) and at 48 hours after subjection to the NEC protocol (control: $n=5$ and NEC: $n=7$ ), both NEC pups and littermate dam fed controls were gavaged with 20 ul of $5 \%$ Evans Blue (in 1.5\% carboxymethycellulose) after 4 hours of fasting. Pups were euthanized 10 minutes after gavage and the total length of the intestine and the length covered by Evans Blue dye were measured (colon excluded). The intestinal transit was expressed as the percentage of small intestinal length covered by Evans Blue.

\section{Statistical analyses}

Statistical analyses were performed using GraphPad Prism 5 (GraphPad Prism, La Jolla, CA, USA). 72-hour survival was analyzed by log-rank test. For the immunohistochemical readouts, data were tested for normality with the D'Agostino and Pearson omnibus normality test. Differences among two groups were measured with a T-test or Mann-Whitney U test and differences among three groups were measured with an ANOVA or non-parametric Kruskal-Wallis test with a post hoc Dunn's test. Differences are regarded as statistically significant at $p<0.05$. Data are presented as median with interquartile range (IQR).

\section{Results}

\section{Histological NEC severity scoring and 72-hour survival}

The animals subjected to the NEC protocol had an increased histological NEC severity score $(p<0.0001$; figure 1$)$ and a significant lower survival than control animals $(p<0.0001$; figure 2). According to the NEC severity scoring, 21 out of 57 animals developed NEC.

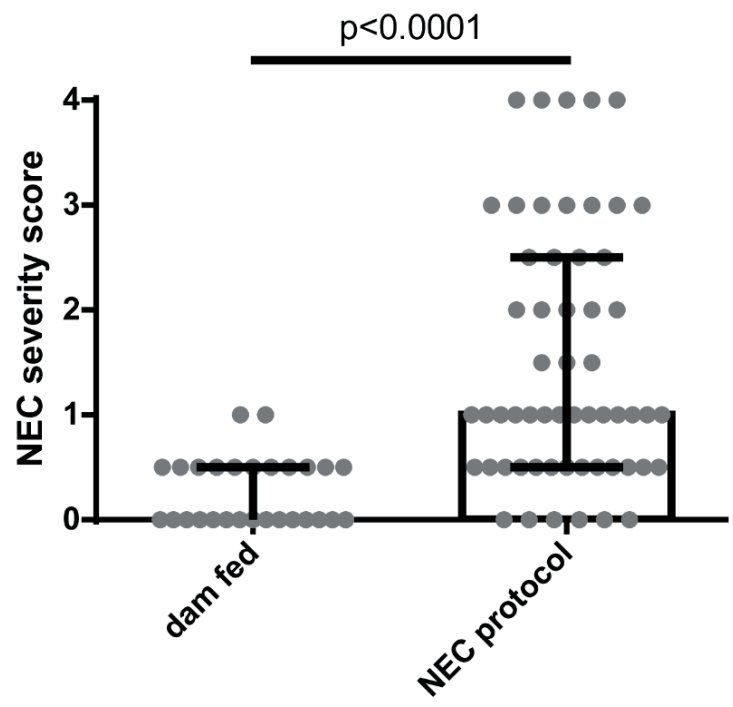

Figure 1. Histological NEC severity score. H\&E slides were scored on a 0 to 4 scale for intestinal damage (23). Increased histological NEC severity score in animals subjected to the NEC protocol, compared to dam fed animals $(\mathrm{p}<0.0001)$. 


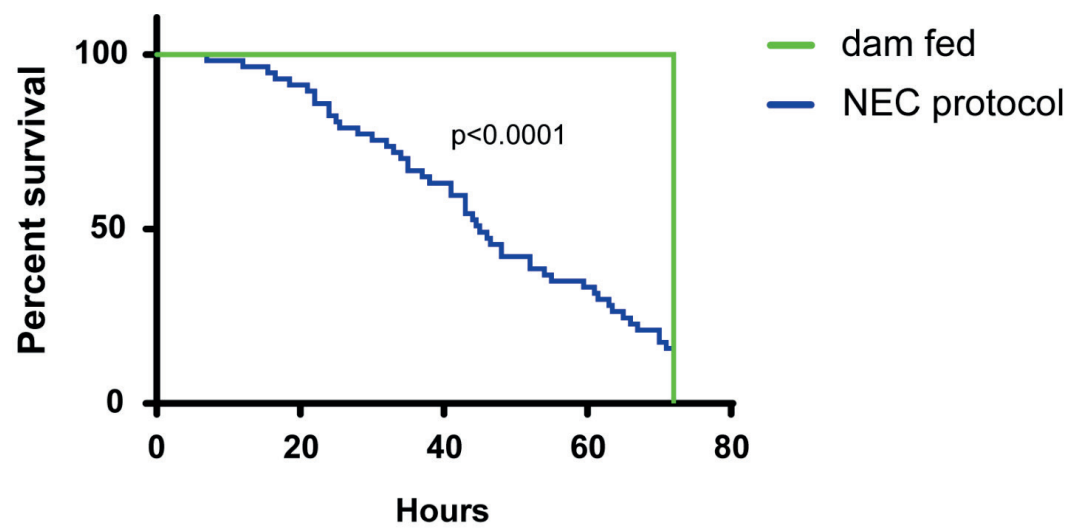

Figure 2. 72-hour survival analyses. The animals subjected to the NEC protocol $(n=57)$ had a significant lower survival than control animals $(n=28)(p<0.0001)$.

\section{ENS alterations}

Doublecortin-positive surface areas (immature neurons) in the submucosal and myenteric plexus were not different between dam fed animals and animals subjected to the NEC protocol (data not shown). The NeuN-positive surface area (mature neurons) in the submucosal and myenteric plexus was increased in animals subjected to the NEC protocol, compared to the control group ( $p=0.02$ and $p=0.0002$; figure 3 ).

A

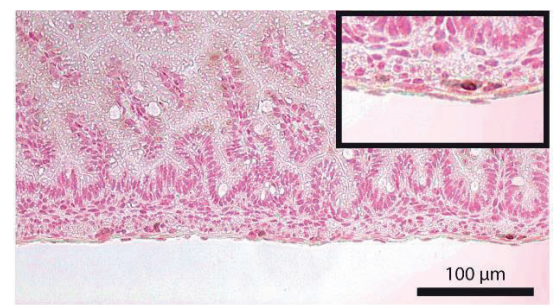

C

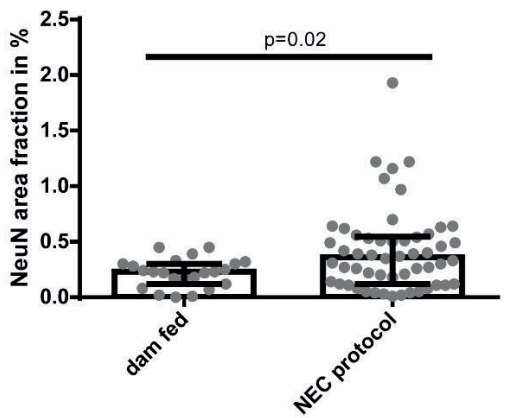

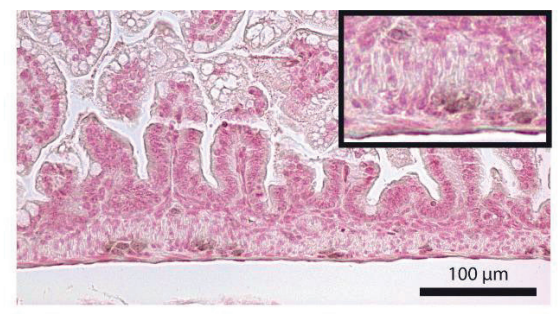

B

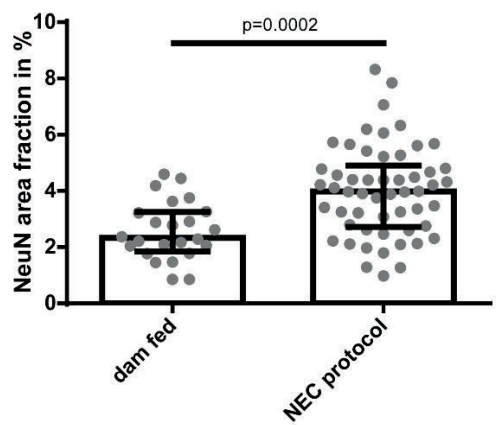

Figure 3. Representative images of NeuN immunoreactivity in the submucosal and myenteric plexus of control animals (A) and animals subjected to the NEC protocol (B). C and D: NeuN-positive surface area was increased in the submucosal and myenteric plexus of animals subjected to the NEC protocol, compared to the control group $(p=0.02$ and $p=0.0002$ ). 
In addition, S100ß-positive surface area (glial cells) in the submucosal and myenteric plexus was increased in animals subjected to the NEC protocol, compared to controls $(p=0.0002$ and $p=0.02$; figure 4$)$.

A

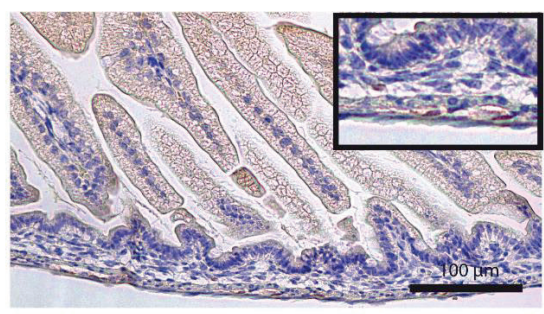

C

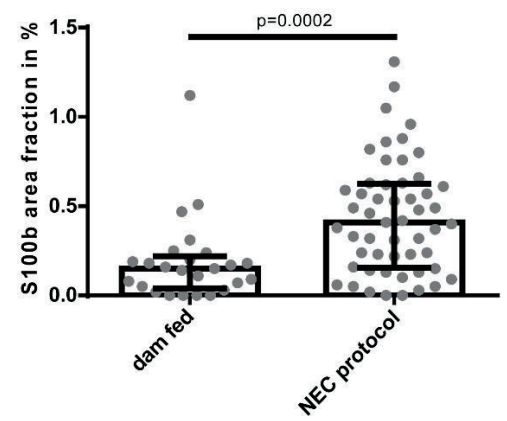

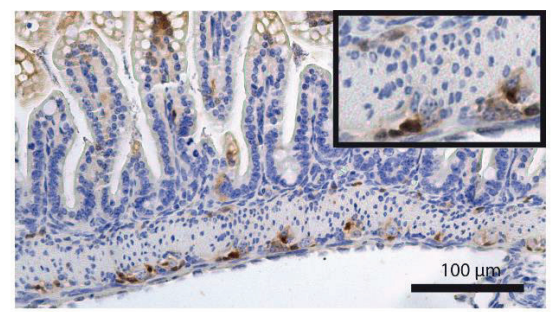

B

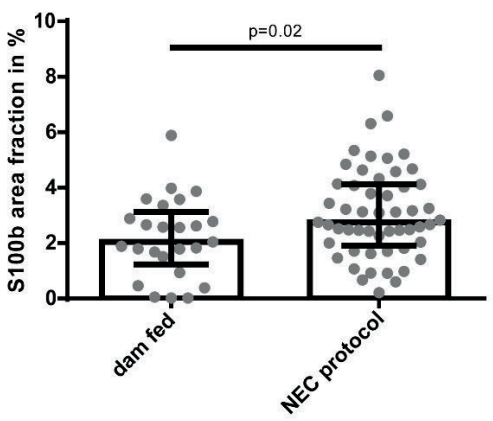

$\mathrm{D}$

Figure 4. Representative images of $S 100 \beta$ immunoreactivity in the submucosal and myenteric plexus of control animals (A) and animals subjected to the NEC protocol (B). C and D: S100ß-positive surface area was increased in the submucosal and myenteric plexus of animals subjected to the NEC protocol, compared to the control group $(p=0.0002$ and $p=0.02)$. 
Finally, the synaptophysin-positive surface area (presynaptic vesicles) in the submucosal and myenteric plexus was increased in animals subjected to the NEC protocol, compared to control (both $p<0.0001$; figure 5).

A

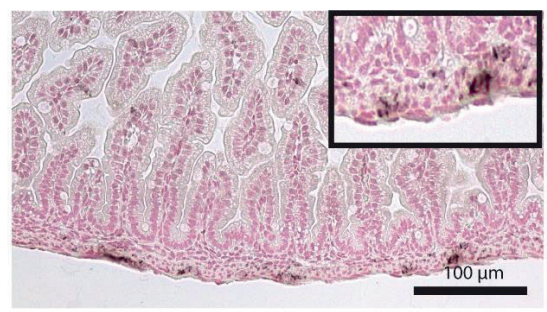

C

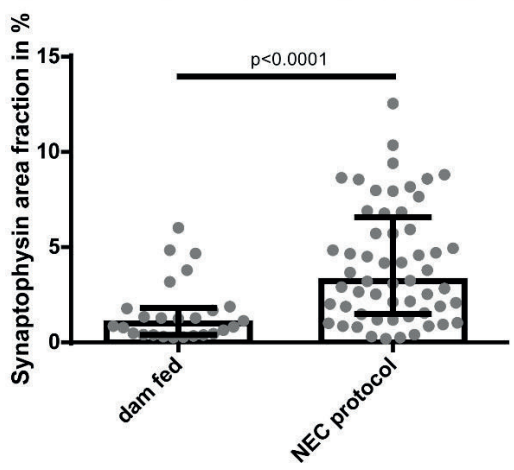

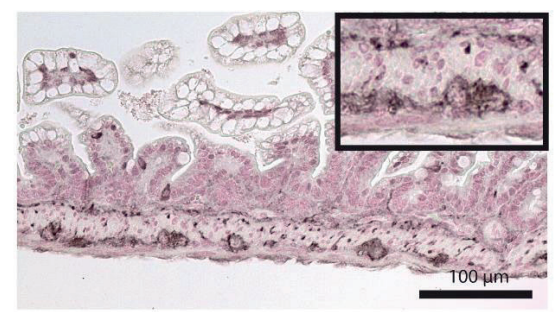

B

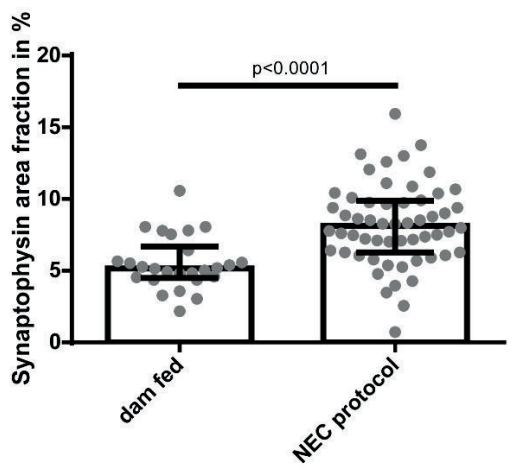

Figure 5. Representative images of synaptophysin immunoreactivity in the submucosal and myenteric plexus of control animals (A) and animals subjected to the NEC protocol (B). C and D: Synaptophysin-positive surface area was increased in the submucosal and myenteric plexus of animals exposed to the NEC protocol, compared to the control group (both $\mathrm{p}<0.0001$ ). 


\section{Intestinal transit}

The intestinal transit was increased in animals 24 hours after subjection to the NEC protocol, compared to control ( $p=0.002$; figure $6 \mathrm{~A})$. At 48 hours after subjection to the NEC protocol, the intestinal transit was decreased, compared to control ( $p=0.01$; figure $6 \mathrm{~B})$.
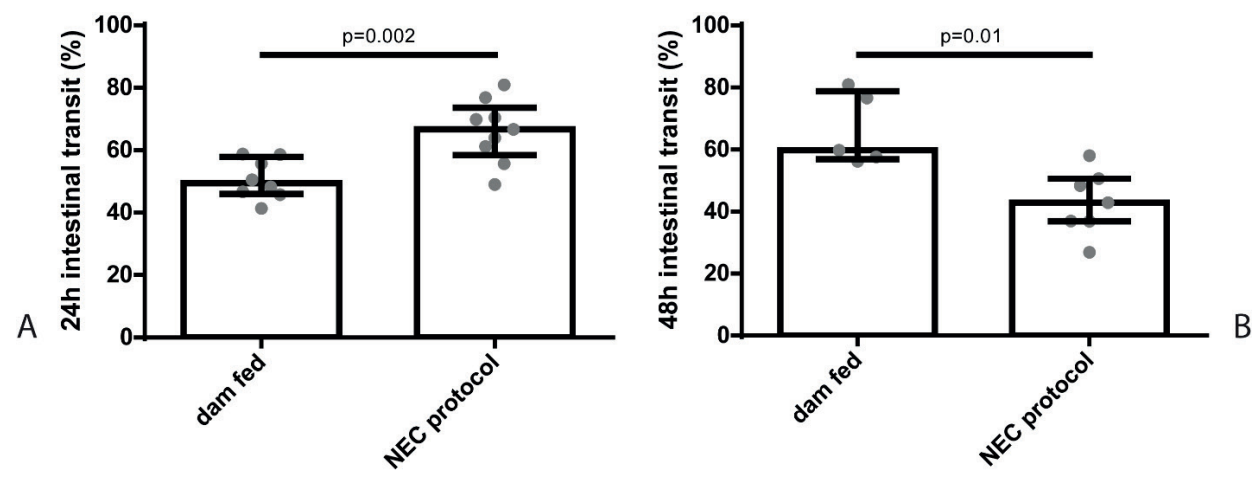

Figure 6. Intestinal transit expressed as the percentage of small intestinal length covered by Evans Blue. A: The intestinal transit was increased in animals 24 hours after subjection to the NEC protocol, compared to control $(p=0.002)$. B: The intestinal transit was decreased in animals 48 hours after subjection to the NEC protocol, compared to control $(p=0.01)$. 


\section{Discussion}

In the current study, we investigated the ENS and intestinal transit in a verified mouse NEC model in which NEC was induced in newborn C57BL/6 mice by formula feeding, oral commensal bacteria with LPS supplementation, asphyxia and cold stress.

We found an increase in mature neurons (NeuN) and enteric glial cells (S100ß) in the mice subjected to the NEC protocol. Enteric glial cells are increasingly recognized for their regulatory function in gastrointestinal homeostasis and their neuroprotective capacities during inflammation $(24,25)$. Ablation of enteric glial cells in a transgenic mouse model leads to a fulminant jejuno-ileitis, resembling NEC, with degeneration of myenteric plexus neurons (26). Thus, the increased S100 $\beta$ surface area in the NEC protocol exposed mice is probably a compensatory response to intestinal inflammation that was earlier shown in this NEC model (27). Interestingly, our findings are in accordance with observations of hypertrophy and hyperplasia of neuronal cell bodies and hyperplasia of glial cells in inflammatory bowel disease, characterized by intestinal inflammation, but typically no necrosis (28).

In contrast, other studies on ENS abnormalities in NEC, both in human specimen (11-13) and a rat NEC model $(13,29)$ report a loss of both glial cells and neurons, with the most severe lesions in the regions prone to intestinal ischemia (12). Moreover, a reduction of neurons was also reported in a mouse NEC model with a different NEC induction protocol, with amongst others a longer exposure time to the experimental NEC protocol of 96 hours (30). Of note, findings of ENS alterations in human NEC are based on specimen obtained during surgery and/or post-mortem, and may not be representative for changes in early and/or less severe NEC in infants. Based on these combined findings, it is tempting to speculate that our data may represent a relatively milder disease state with an ENS response that predisposes to glial cell and neuronal loss at a later time point. This suggests that different rodent NEC models and different durations of post NEC induction follow up can be used to gain longitudinal insight in the ENS changes during NEC pathophysiology. To extrapolate these findings to the clinical setting, functional ENS analyses, such as measurements of transit time and intestinal motility are of vital importance.

In addition, we observed an increased synaptophysin-positive surface area in the submucosal and myenteric plexus of animals subjected to the NEC protocol, suggesting increased production and potential enhanced neurotransmitter release. Importantly, similar changes were already detected in utero in the context of perinatal asphyxia (31), a known risk factor for NEC development (32). A plausible mechanistic explanation for the increase in synaptophysin expression is provided by in vitro work from Bottner et al., reporting that stimulating a rat enteric nerve cell culture with glial-cell line derived neurotrophic factor (GDNF) increased synaptophysin mRNA expression (33). GDNF is produced by glial cells, and its expression is upregulated during inflammation (34), as is induced during this experimental NEC protocol (27). Whether the increased presence of presynaptic vesicles during NEC alters ENS signaling and may contribute to or protect against NEC development remains to be elucidated. 
Importantly, we observed an increased intestinal transit in animals subjected to the NEC protocol for 24 hours, indicating increased intestinal motility. This most likely results from an immunological host response and subsequent diarrhea (35-38). Alternatively, this might reflect a functional consequence of the increased presence of presynaptic vesicles (synaptophysin immunoreactivity), although this would be a rather quick response. In contrast, after 48 hours of exposure to the NEC protocol, the intestinal transit decreased, implying decreased intestinal motility in animals subjected to the NEC protocol. Possibly, neurons were initially able to compensate and react to intestinal inflammation, but in a later stage, despite the neurons still being able to produce synaptophysin, motility decreased. These findings indicate that additional studies are needed to unravel the mechanisms behind the altered intestinal motility following exposure to the NEC protocol. In addition, it shows that including a functional assay of gut motility is of importance when studying ENS changes in NEC.

A limitation of this study is that, due to the size of the mouse intestines, we could not distinguish between the different anatomical parts of the small intestine, or varying disease severities within parts of a single intestine. Moreover, the translation of these rodent findings into the clinic is challenging as biopsies from NEC patients, that are available for research, exclusively reflect children with the most severe phenotype of the NEC spectrum.

In summary, in this experimental NEC model, subjection to the experimental NEC protocol was associated with an increase of mature neurons, glial cells and increased presence of presynaptic vesicles in the submucosal and myenteric plexus and time-dependant changes in intestinal motility. As the current histological data cannot fully explain the observed changes in motility, additional studies are needed to unravel the mechanisms underlying these functional changes. The ENS changes in this NEC model seem to represent a relatively milder disease state within the clinical NEC spectrum, with an ENS response that precedes glial cell and neuronal loss. 


\section{References}

1. Lin PW, Stoll BJ. Necrotising enterocolitis. Lancet. 2006;368(9543):1271-83 DOI: 10.1016/S01406736(06)69525-1.

2. Ahle $M$, Drott $P$, Andersson RE. Epidemiology and trends of necrotizing enterocolitis in Sweden: 1987-2009. Pediatrics. 2013;132(2):e443-51 DOI: 10.1542/peds.2012-3847.

3. Heida FH, Stolwijk L, Loos MH, van den Ende SJ, Onland W, van den Dungen FA, Kooi EM, Bos AF, Hulscher JB, Bakx R. Increased incidence of necrotizing enterocolitis in the Netherlands after implementation of the new Dutch guideline for active treatment in extremely preterm infants: Results from three academic referral centers. J Pediatr Surg. 2017;52(2):273-6 DOI: 10.1016/j. jpedsurg.2016.11.024.

4. Blakely ML, Lally KP, McDonald S, Brown RL, Barnhart DC, Ricketts RR, Thompson WR, Scherer LR, Klein MD, Letton RW, Chwals WJ, Touloukian RJ, Kurkchubasche AG, Skinner MA, Moss $\mathrm{RL}$, Hilfiker ML, Network NECSotNNR. Postoperative outcomes of extremely low birth-weight infants with necrotizing enterocolitis or isolated intestinal perforation: a prospective cohort study by the NICHD Neonatal Research Network. Ann Surg. 2005;241(6):984-9; discussion 9-94 DOI: 10.1097/01.sla.0000164181.67862.7f.

5. Hull MA, Fisher JG, Gutierrez IM, Jones BA, Kang KH, Kenny M, Zurakowski D, Modi BP, Horbar JD, Jaksic T. Mortality and management of surgical necrotizing enterocolitis in very low birth weight neonates: a prospective cohort study. J Am Coll Surg. 2014;218(6):1148-55 DOI: 10.1016/j. jamcollsurg.2013.11.015.

6. Hintz SR, Kendrick DE, Stoll BJ, Vohr BR, Fanaroff AA, Donovan EF, Poole WK, Blakely ML, Wright L, Higgins R. Neurodevelopmental and growth outcomes of extremely low birth weight infants after necrotizing enterocolitis. Pediatrics. 2005;115(3):696-703 DOI: 10.1542/peds.2004-0569.

7. Hong CR, Fullerton BS, Mercier CE, Morrow KA, Edwards EM, Ferrelli KR, Soll RF, Modi BP, Horbar JD, Jaksic T. Growth morbidity in extremely low birth weight survivors of necrotizing enterocolitis at discharge and two-year follow-up. J Pediatr Surg. 2018;53(6):1197-202 DOI: 10.1016/j. jpedsurg.2018.02.085.

8. Rees CM, Pierro A, Eaton S. Neurodevelopmental outcomes of neonates with medically and surgically treated necrotizing enterocolitis. Archives of disease in childhood Fetal and neonatal edition. 2007;92(3):F193-F8 DOI: 10.1136/adc.2006.099929.

9. Schulzke SM, Deshpande GC, Patole SK. Neurodevelopmental outcomes of very low-birth-weight infants with necrotizing enterocolitis: a systematic review of observational studies. Arch Pediatr Adolesc Med. 2007;161(6):583-90 DOI: 10.1001/archpedi.161.6.583.

10. Neu J, Walker WA. Necrotizing enterocolitis. N Engl J Med. 2011;364(3):255-64 DOI: 10.1056/ NEJMra1005408.

11. Sigge W, Wedel T, Kuhnel W, Krammer HJ. Morphologic alterations of the enteric nervous system and deficiency of non-adrenergic non-cholinergic inhibitory innervation in neonatal necrotizing enterocolitis. Eur J Pediatr Surg. 1998;8(2):87-94 DOI: 10.1055/s-2008-1071128.

12. Wedel T, Krammer HJ, Kuhnel W, Sigge W. Alterations of the enteric nervous system in neonatal necrotizing enterocolitis revealed by whole-mount immunohistochemistry. Pediatr Pathol Lab Med. 1998;18(1):57-70.

13. Zhou Y, Yang J, Watkins DJ, Boomer LA, Matthews MA, Su Y, Besner GE. Enteric nervous system abnormalities are present in human necrotizing enterocolitis: potential neurotransplantation therapy. Stem Cell Res Ther. 2013;4(6):157- DOI: 10.1186/scrt387. 
14. Zhou Y, Yang J, Watkins DJ, Boomer LA, Matthews MA, Su Y, Besner GE. Enteric nervous system abnormalities are present in human necrotizing enterocolitis: potential neurotransplantation therapy. Stem Cell Res Ther. 2013;4(6):157 DOI: 10.1186/scrt387.

15. Chen W, Sun J, Kappel SS, Gormsen M, Sangild PT, Aunsholt L. Gut transit time, using radiological contrast imaging, to predict early signs of necrotizing enterocolitis. Pediatr Res. 2020 DOI: 10.1038/ s41390-020-0871-0.

16. Goyal RK, Hirano I. The enteric nervous system. N Engl J Med. 1996;334(17):1106-15 DOI: 10.1056/ nejm199604253341707.

17. von Boyen GB, Steinkamp M, Reinshagen M, Schafer KH, Adler G, Kirsch J. Proinflammatory cytokines increase glial fibrillary acidic protein expression in enteric glia. Gut. 2004;53(2):222-8.

18. Yoo BB, Mazmanian SK. The Enteric Network: Interactions between the Immune and Nervous Systems of the Gut. Immunity. 2017;46(6):910-26 DOI: 10.1016/j.immuni.2017.05.011.

19. Burns AJ, Roberts RR, Bornstein JC, Young HM. Development of the enteric nervous system and its role in intestinal motility during fetal and early postnatal stages. Semin Pediatr Surg. 2009;18(4):196-205 DOI: 10.1053/j.sempedsurg.2009.07.001.

20. Lake J, Heuckeroth RO. Entericnervous system development:migration, differentiation, and disease. Am J Physiol Gastrointest Liver Physiol. 2013;305(1):G1-24 DOI: 10.1152/ajpgi.00452.2012.

21. Sulistyo A, Rahman A, Biouss G, Antounians L, Zani A. Animal models of necrotizing enterocolitis: review of the literature and state of the art. Innov Surg Sci. 2018;3(2):87-92 DOI: 10.1515/iss-20170050.

22. Ganji N, Li B, Lee C, Filler R, Pierro A. Necrotizing Enterocolitis: State of the Art in Translating Experimental Research to the Bedside. Eur J Pediatr Surg. 2019;29(4):352-60 DOI: 10.1055/s-00391693994.

23. Tian R, Liu SX, Williams C, Soltau TD, Dimmitt R, Zheng X, De Plaen IG. Characterization of a necrotizing enterocolitis model in newborn mice. Int J Clin Exp Med. 2010;3(4):293-302.

24. Sharkey KA. Emerging roles for enteric glia in gastrointestinal disorders. J Clin Invest. 2015;125(3):918-25 DOI: 10.1172/jci76303.

25. Margolis KG, Gershon MD. Enteric Neuronal Regulation of Intestinal Inflammation. Trends Neurosci. 2016;39(9):614-24 DOI: 10.1016/j.tins.2016.06.007.

26. Bush TG, Savidge TC, Freeman TC, Cox HJ, Campbell EA, Mucke L, Johnson MH, Sofroniew MV. Fulminant jejuno-ileitis following ablation of enteric glia in adult transgenic mice. Cell. 1998;93(2):189-201 DOI: 10.1016/s0092-8674(00)81571-8.

27. Managlia E, Liu SXL, Yan X, Tan XD, Chou PM, BarrettTA, De Plaen IG. Blocking NF-kappaBActivation in Ly6c(+) Monocytes Attenuates Necrotizing Enterocolitis. Am J Pathol. 2019;189(3):604-18 DOI: 10.1016/j.ajpath.2018.11.015.

28. Geboes K, Collins S. Structural abnormalities of the nervous system in Crohn's disease and ulcerative colitis. Neurogastroenterol Motil. 1998;10(3):189-202.

29. Zhou Y, Wang Y, Olson J, Yang J, Besner GE. Heparin-binding EGF-like growth factor promotes neuronal nitric oxide synthase expression and protects the enteric nervous system after necrotizing enterocolitis. Pediatr Res. 2017;82(3):490-500 DOI: 10.1038/pr.2017.68.

30. Wei J, Zhou Y, Besner GE. Heparin-binding EGF-like growth factor and enteric neural stem cell transplantation in the prevention of experimental necrotizing enterocolitis in mice. Pediatr Res. 2015;78(1):29-37 DOI: 10.1038/pr.2015.63. 
31. Nikiforou M, Willburger C, de Jong AE, Kloosterboer N, Jellema RK, Ophelders DRMG, Steinbusch HWM, Kramer BW, Wolfs T. Global hypoxia-ischemia induced inflammation and structural changes in the preterm ovine gut which were not ameliorated by mesenchymal stem cell treatment. Molecular medicine (Cambridge, Mass). 2016;22:244-57 DOI: 10.2119/molmed.2015.00252.

32. Fox TP, Godavitarne C. What really causes necrotising enterocolitis? ISRN Gastroenterol. 2012;2012:628317- DOI: 10.5402/2012/628317.

33. Bottner M, Harde J, Barrenschee M, Hellwig I, Vogel I, Ebsen M, Wedel T. GDNF induces synaptic vesicle markers in enteric neurons. Neurosci Res. 2013;77(3):128-36 DOI: 10.1016/j. neures.2013.08.012.

34. von Boyen GB, Schulte N, Pfluger C, Spaniol U, Hartmann C, Steinkamp M. Distribution of enteric glia and GDNF during gut inflammation. BMC Gastroenterol. 2011;11:3 DOI: 10.1186/1471-230x11-3.

35. Wirthlin DJ, Cullen JJ, Spates ST, Conklin JL, Murray J, Caropreso DK, Ephgrave KS. Gastrointestinal transit during endotoxemia: the role of nitric oxide. J Surg Res. 1996;60(2):307-11 DOI: 10.1006/ jsre.1996.0048.

36. Hellstrom PM, al-Saffar A, Ljung T, Theodorsson E. Endotoxin actions on myoelectric activity, transit, and neuropeptides in the gut. Role of nitric oxide. Dig Dis Sci. 1997;42(8):1640-51 DOI: 10.1023/a:1018897028463.

37. Cullen JJ, Doty RC, Ephgrave KS, Hinkhouse MM, Broadhurst K. Changes in intestinal transit and absorption during endotoxemia are dose dependent. J Surg Res. 1999;81(1):81-6 DOI: 10.1006/ jsre.1998.5452.

38. Cullen JJ, Mercer D, Hinkhouse M, Ephgrave KS, Conklin JL. Effects of endotoxin on regulation of intestinal smooth muscle nitric oxide synthase and intestinal transit. Surgery. 1999;125(3):339-44. 


$$
-5
$$




\section{Chorioamnionitis induces hepatic inflammation and time-dependent changes of the enterohepatic circulation in the ovine fetus}

C. Heymans, M. den Dulk, K. Lenaerts, L.R. Heij, I.H. de Lange, M. Hadfoune, C. van Heugten, B.W. Kramer, A.H. Jobe, M. Saito, M.W. Kemp, T.G.A.M. Wolfs, W.G. van Gemert 


\section{Abstract}

\section{Background}

Chorioamnionitis, inflammation of fetal membranes, is an important cause of preterm birth and a risk factor for the development of adverse neonatal outcomes including sepsis and intestinal pathologies. Intestinal bile acids (BAs) accumulation and hepatic cytokine production are involved in adverse intestinal outcomes. These findings triggered us to study the liver and enterohepatic circulation (EHC) following intra-amniotic (IA) lipopolysaccharide (LPS) exposure.

\section{Materials and Methods}

An ovine chorioamnionitis model was used in which circulatory cytokines and outcomes of the liver and EHC of preterm lambs were longitudinally assessed following IA administration of $10 \mathrm{mg}$ LPS at 5,12 or $24 \mathrm{~h}$ or $2,4,8$ or $15 \mathrm{~d}$ before preterm birth.

\section{Results}

Hepatic inflammation was observed, characterized by increased hepatic cytokine mRNA levels ( $5 h-2 d$ post IA LPS exposure) and increased erythropoietic clusters (at 8 and $15 d$ post IA LPS exposure). Besides, $12 \mathrm{~h}$ after IA LPS exposure, plasma BA levels were increased, whereas gene expression levels of several hepatic BA transporters were decreased. Initial EHC alterations normalized over time.

\section{Conclusion}

IA LPS exposure induces significant time-dependent changes in the fetal liver and EHC. These chorioamnionitis-induced changes have potential postnatal consequences and the duration of IA LPS exposure might be essential herein. 


\section{Introduction}

Premature birth, before 37 weeks of gestation, is a major cause of morbidity and mortality among infants worldwide (1). Chorioamnionitis, inflammation of the chorion and amnion during pregnancy, resulting from an ascending bacterial infection through the birth canal, is associated with premature birth (2-4). During chorioamnionitis, the fetus can develop the fetal inflammatory response syndrome (FIRS), characterized by increased fetal systemic IL-6 and IL-8 levels (5). Chorioamnionitis, prematurity and FIRS can contribute to significant rates of neonatal morbidity and mortality and are associated with the development of adverse neonatal outcomes including early-onset sepsis and intestinal pathologies (6-9).

Sepsis-associated cholestasis is a common complication in infants and is commonly caused by lipopolysaccharide (LPS), derived from Gram negative bacteria (10). This inflammationinduced cholestasis results from a reduced mRNA expression of various hepatic bile acid (BA) transporters, such as the Na+-taurocholate cotransporting polypeptide (NTCP) and the bile salt export pump (BSEP) (10). In specific, LPS activates cytokine production of Kupffer cells such as tumor necrosis factor alpha (TNF- $\alpha$ ) and interleukin 1 beta (IL-1 $\beta$ ). These cytokines bind to their receptors on hepatocytes that activates an intracellular signaling cascade resulting in altered nuclear transcription factors and reduced mRNA expression (10). Cholestasis can lead to hepatocyte injury as BAs have been shown to be hepatotoxic during cholestasis (11). Several mechanisms may account for this hepatotoxity; BAs could disrupt cell membranes through their detergent action on lipid components and also promote the generation of reactive oxygen species that oxidatively modify lipids, proteins and nucleic acids, which eventually cause hepatocyte necrosis and apoptosis (11).

The liver and intestine are closely connected via the enterohepatic circulation (EHC) of BAs. In line, BAs are recently found to be critical regulators of intestinal epithelial function (12). Several studies have found that elevated ileal BAs and an altered expression of several BAs transporters may result in ileal damage, contributing to the development of necrotizing enterocolitis (NEC). More precisely, full necrosis in the ileum was found in rats as the result of intraluminal accumulation of conjugated BAs, which was similar to the histopathological findings in an experimental rat NEC model $(13,14)$. Increased BA synthesis might be the cause of these elevated intraluminal BAs concentrations (15). In addition, preterm infants with NEC and rodents with induced NEC were found to have an increased expression of the apical sodium-dependent bile acid transporter (ASBT), a protein involved in intestinal BAs uptake, suggesting increased BAs uptake by enterocytes $(14,16)$. In addition, an insufficient transport from the apical to basolateral side of the enterocyte was suggested since rats with induced NEC had a decreased expression of ileal bile acid-binding protein (IBABP) in their ileum (14). Altogether, this results in BAs accumulation within enterocytes, with concomitant enterocyte damage (17), resulting from similar mechanisms as in cholestasis induced hepatocyte injury (11). The production of hepatic cytokines was increased in neonatal rats with induced NEC and this correlated with the progression of intestinal damage during NEC development (18), which further underlines the important role of the gut-liver axis in NEC pathogenesis. 
Fecal BA levels were found to be higher in preterm infants in the week preceding NEC manifestation compared with gestation matched controls (17). Moreover, it was shown that fetuses exposed to endotoxin-induced chorioamnionitis develop hepatic inflammation and a disturbed lipid metabolism in utero (19), which may persist into adolescence (20). These combined findings suggest that the earlier mentioned liver and EHC alterations might already have their origin in utero.

Recent research showed that the fetal liver is an active immune organ with the ability of inducing an early and robust innate immune response activation, and immune activation is already initiated within 1 hour to 5 hours after an in utero inflammatory challenge (21). The changes during intra-uterine infection, including liver inflammation and EHC alterations, are likely to be time-dependent as inflammation is a dynamic process and the vulnerability of the fetus to injurious hits during the complex intra-uterine development varies. The aim of this study is, given the involvement of the liver in sepsis and adverse gastro-intestinal outcomes, to evaluate the time-dependent effects of intra-uterine administration of one bolus of LPS from 15 days to 5 hours before premature delivery, on the liver and EHC of premature sheep.

\section{Materials and Methods}

\section{Animal model and experimental procedures}

This animal study was performed in Australia and the experiments were approved by the Animal Ethics Committees at The University of Western Australia (Perth, Australia; permit number: RA/3/100/928).

The original ovine model and experimental design were previously published $(5,22-$ 24). In short, date-mated merino ewes pregnant with singleton fetuses were randomly assigned to eight different groups. After dropout or exclusion (fetuses in the control group with extremely high systemic IL- 6 and/or IL-8 levels), the different groups consisted of 6 to 7 animals per group. The pregnant ewes received a single intra-amniotic (IA) injection under ultrasound guidance of $10 \mathrm{mg}$ Escherichia coli-derived lipopolysaccharide (LPS) (O55:B5; Sigma-Aldrich, St. Louis, MO, USA) dissolved in saline. These injections were given at 5,12 or 24 hours or $2,4,8$ or 15 days before preterm delivery at 125 days of gestation (corresponds with 30-32 weeks of human gestation; term gestation in sheep is around 150 days). The control group received IA injections of saline at variable gestational ages comparable to the time points of the LPS injections before preterm delivery (Figure 1). 


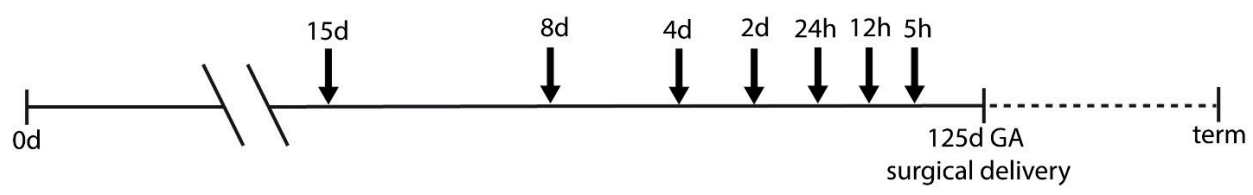

Figure 1. Study design. At 5, 12, or 24 hours or 2, 4, 8 or 15 days (black arrows) pregnant ewes received an IA injection with $10 \mathrm{mg}$ LPS before preterm delivery at 125 days of gestation (term $\sim 150$ days). An IA saline injection at comparable time points to LPS injections was given to control animals. Timing shown in gestational days.

The preterm lambs were delivered by cesarean section at 125 days of gestation and euthanized with intravenous pentobarbital (100 mg/kg). During necropsy, blood, liver and terminal ileum samples were sampled. Liver and ileum samples were fixed in paraformaldehyde and subsequently embedded in paraffin or liver and ileum samples were snap frozen in nitrogen.

\section{Qualitative analysis of liver histology}

A Hematoxylin and Eosin (H\&E) staining was performed which an independent pathologist blinded to the experimental set-up analyzed as previously described (25). In short, the slides were qualitatively scored pathologically on a 0 to 4 scale for hepatic sinusoidal dilatation, shape and size of central veins and number and location of extramedullary hematopoietic clusters. Animals that had been assigned with scoring 0 had no sinusoidal dilatation, no divergent shape or size of central veins and no altered extramedullary hematopoietic clusters. Animals that had been assigned with scoring 4 had pronounced sinusoidal dilatation throughout the parenchyma, large central veins with venous stowing throughout the parenchyma and a severely increased pathologic score of extramedullary hematopoietic clusters. An increased pathologic score of extramedullary hematopoiesis, reflecting clustered and conflated hepatic erythropoiesis in the parenchyma, is a hallmark of increased erythropoiesis as a response to inflammation (26).

\section{Total bile acid assay}

As previously described (25), total bile acids (tBAs) were measured in plasma, liver homogenate and terminal ileum homogenate by an enzymatic cycling method, according to the manufacture protocol (Total Bile Acids Assay kit, Diazyme Laboratories, Poway, CA, USA). tBAs in liver and ileum homogenate were corrected for protein content, which were measured with a BCA protein assay kit (Thermo Fisher Scientific, Waltham, MA, USA).

\section{RNA extraction and real-time PCR}

RNA extraction and quantitative real-time polymerase chain reaction (qPCR) was performed as previously described (25). In short, RNA was extracted from snap frozen liver and terminal ileal tissue using TRI reagent (Thermo Fisher Scientific)/chloroform extraction. Hereafter, with the use of a sensifast cDNA Synthesis kit (Bioline, London, UK), RNA was reverse transcribed into CDNA. With the specific primer in Sensimix SYBR \& Fluorescein Kit (Bioline), a qPCR was performed using a 384-wells qPCR plate. qPCR reactions were executed in a LightCycler 480 Instrument (Roche Applied Science, Basel, Switzerland) for 45 cycles. mRNA expression levels of tumor necrosis factor alpha 
(TNF-a), interleukin 1 beta (IL-1 $\beta$ ), interleukin-8 (IL-8) and interleukin-18 (IL-18) were measured to assess liver inflammation. IL-18 mRNA expression was also measured in ileal samples to asses ileal inflammation. Gene expression levels of cholesterol 7 alphahydroxylase (CYP7A1), cytochrome P450 family 27 subfamily A member 1 (CYP27A1), $\mathrm{Na}$-taurocholate cotransporting polypeptide (NTCP), bile salt export pump (BSEP), apical sodium-dependent bile acid transporter (ASBT), fibroblast growth factor 19 (FGF19), ileal bile acid-binding protein (IBABP) and organic solute transporter alpha-beta (OSTa- $\beta$ ) were measured to assess alterations in the EHC of BAs. To calculate the mRNA expression levels, LinRegPCR software (version 2016.0, Heart Failure Research Center, Academic Medical Center, Amsterdam, the Netherlands) was used. The geometric mean of the expression levels of three reference genes (ribosomal protein S15 (RPS15), glyceraldehyde 3-phosphate dehydrogenase (GAPDH) and peptidylprolyl isomerase A (PPIA)) was calculated and used as a normalization factor. Data are shown as fold increase over the control value; arbitrary unit (AU). Primer sequences are displayed in Table 1.

Table 1. Primer sequences

\begin{tabular}{|c|c|c|}
\hline Primer & Forward & Reverse \\
\hline RPS15 & 5'-CGAGATGGTGGGCAGCAT-3' & 5'-GCTTGATTTCCACCTGGTTGA-3' \\
\hline GAPDH & 5'-GGAAGCTCACTGGCATGGC-3' & 5'-CCTGCTTCACCACCTTCTTG-3' \\
\hline PPIA & 5'-TTATAAAGGTTCCTGCTTTCACAGAA-3' & 5'-ATGGACTTGCCACCAGTACCA-3' \\
\hline TNF-a & 5'-CATCTTCTCAAGCCTCAAATAACAA-3' & 5'-TGCGAGTAGATGAGGTAAAGCCC-3' \\
\hline $\mathrm{IL}-1 \beta$ & 5'-AGAATGAGCTGTTATTTGAGGTTGATG-3' & 5'-GTGAGAAATCTGCAGCTGGATGT-3' \\
\hline IL-8 & 5'-GTTCCAAGCTGGCTGTTGCT-3' & 5'-GTGGAAAGGTGTGGAATGTGTTT-3' \\
\hline IL-18 & 5'- AAGGGGCTGCCGTCTTCTAT-3' & 5'- GATCTGATTCCAGGTCGCCAT-3' \\
\hline CYP7A1 & 5'-GGGCATCACAAGCAAACACC-3' & 5'-GATGATACTGTCTAGCACGGG-3' \\
\hline CYP27A1 & 5'- CCCAAGAATACCCAGTTTGTGC-3' & 5'- GGTGGCAGAAGACTCAGTTCA-3' \\
\hline NTCP & 5'-TCCTCAAATCCAAACGGCCA-3' & 5'-GTTTGGATCGTCCATTGAGGC-3' \\
\hline BSEP & 5'-ACTCAGTAATTCTTCGCAGTGTG-3' & 5'-ATCGAAACAATCGAAAGAAGCCA-3' \\
\hline ASBT & 5'-CATGGACCTGAGCGTCAGCAT-3' & 5'-CACGGAGACGGGAACAACAA-3' \\
\hline FGF19 & 5'-TTGATGGAGATCAGGGCGGT-3' & 5'-CGGATCTCCTCCTCGAAAGC-3' \\
\hline IBABP & 5'-ACAAGAAGTTCAAGGTCACCG-3' & 5'-TGATACGGCTTTATGGCCCC-3' \\
\hline OSTa & 5'-ATCCCAGGTACACGGCAGAT-3' & 5'-ATTGAGGCCAGGACAAGCAA-3' \\
\hline OST $\beta$ & 5'-CCGAGTAGAGGATGCAACTCC-3' & 5'-TTTGTTTTTCCGGTGGCAGC-3' \\
\hline
\end{tabular}

\section{Immunohistochemistry}

Staining of ionized calcium binding adaptor molecule 1 (IBA1), a macrophage marker, in the terminal ileum was used as a marker for intestinal inflammation. For immunohistochemistry, the following antibodies were used: polyclonal rabbit anti-rat IBA1 (019-19741; Fujifilm Wako Chemicals Europe, Neuss, Germany) and polyclonal swine anti-rabbit biotin (E0353; DakoCytomation, Glostrup, Denmark). After 4\% paraformaldehyde fixation and paraffin embedding $4 \mu \mathrm{m}$ thick sections were cut. Following rehydration, endogenous peroxidase activity was blocked by incubating sections for 20 minutes in $0.3 \% \mathrm{H} 2 \mathrm{O} 2$ dissolved in phosphatase buffered saline. Antigen retrieval was performed by boiling the slides in 
$10 \mathrm{mM}$ sodium citrate buffer ( $\mathrm{pH}$ 6.0). Thereafter, aspecific binding sites were blocked by incubating the sections with $4 \%$ normal goat serum and sections were incubated with the primary antibody overnight at $4{ }^{\circ} \mathrm{C}$. Subsequently, sections were incubated with biotin-conjugated secondary antibody for 1 hour at room temperature. IBA1 positive cells were detected with 3,3'-diaminobenzidine ([DAB]; Sigma Aldrich, St Louis, MO, USA). IBA1 immunoreactivity (IBA1 positive surface area) was detected and corrected for ileal surface area with the use of Qwin Pro software (v3.4.0, Leica Microsystem, Wetzlar, Germany). IBA1 immunoreactivity is depicted as the percentage of ileal surface area positively stained for IBA1.

\section{Data Analysis}

Data are presented as median with interquartile range (IQR). Statistical analyses were carried out with GraphPad Prism (version 6.01, GraphPad Software Inc., La Jolla, CA, USA). To analyze significant differences between the groups, a nonparametric KruskalWallis test followed by Dunn's post hoc test was performed. At $p \leq 0.05$, differences were considered statistically significant. Given the small study groups and the potential biological relevance, differences with a $\mathrm{p}<0.10$ are also taken into account, and described as tendencies as previously described (27). This assumption will increase the chance of a type I error, but will decrease the chance of a type II error. 


\section{Results}

\section{Severe liver inflammation at day $\mathbf{8}$ and day $\mathbf{1 5}$ after IA LPS administration}

Histologically, no sinusoidal dilatation or divergent shape or size of central veins was observed in any of the groups. An increased qualitative pathological score of extramedullary hematopoiesiswas observed in the liver of animals 8 days and 15 days after LPS administration, compared to control (both $p<0.05$; Figure $2 a+b+c+d$ ), reflecting clustered and conflated erythropoiesis in the liver parenchyma (Figure $2 \mathrm{~b}$ and $2 \mathrm{c}$ ).

A

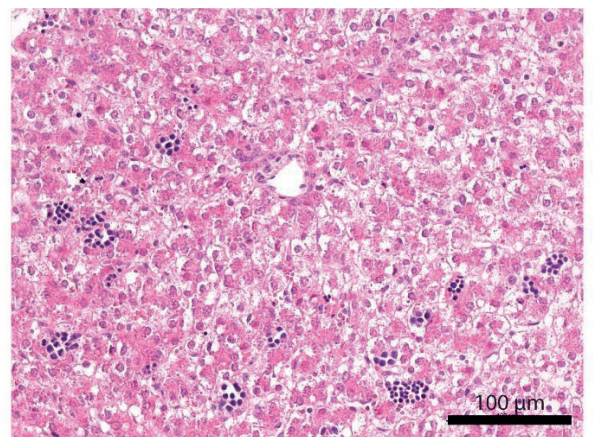

C

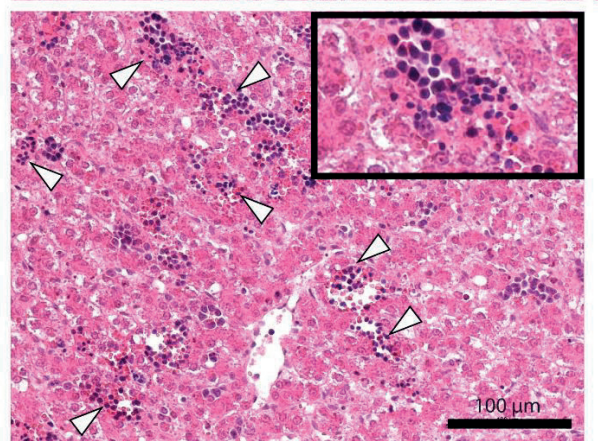

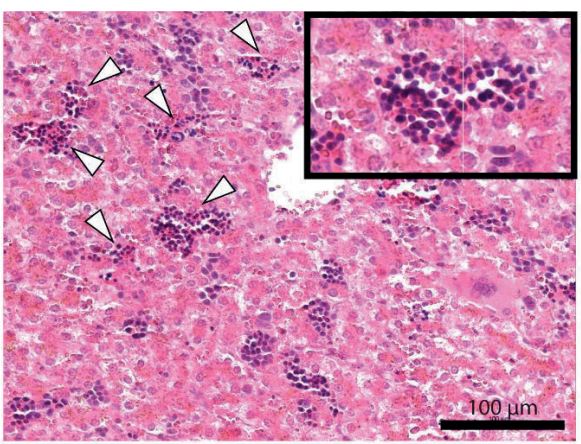

B

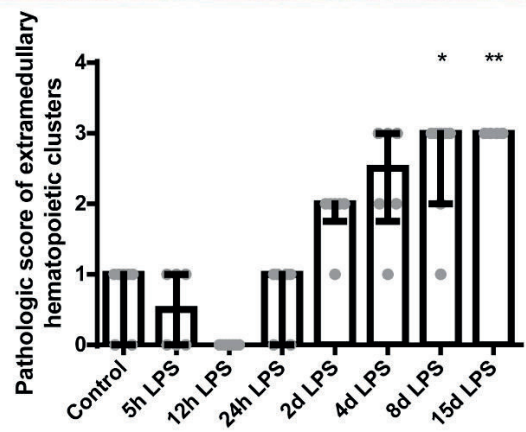

Figure 2. Scoring of H\&E slides on a 0 to 4 scale for degree of sinusoidal dilatation, shape and size of central veins and number and location of extramedullary hematopoietic clusters. Representative images of control (a), at day 8 after IA LPS administration (b) and at day 15 after LPS administration (c). Data are presented as median with IQR (d). D: Pathologic score with increased number of extramedullary hematopoietic clusters in the animals at day 8 and day 15 after IA LPS administration. Specifically, the increased extramedullary hematopoiesis, which manifests as clustering and conflation of hepatic erythropoiesis in the parenchyma, is indicated by white triangles $(b+c) .{ }^{*} p<0.05$ and ${ }^{* *} p<0.01$ compared to control.

Hepatic IL-8 mRNA levels tended to be increased 5 hours after IA LPS administration, compared to control ( $p=0.09$; Figure 3a). Furthermore, TNF-a mRNA levels tended to be increased 12 hours after IA LPS, compared to control ( $p=0.07$; Figure $3 b)$. Moreover, IL-18 mRNA levels were increased 24 hours and 2 days after IA LPS administration (both $p \leq 0.05$; Figure 3c). Hepatic IL-1 $\beta$ mRNA levels did not differ between the groups (data not shown). 

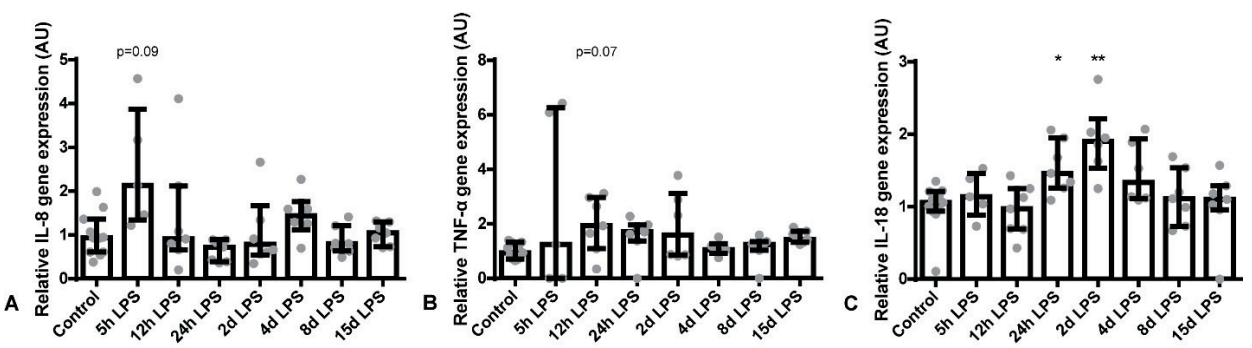

Figure 3. Relative mRNA expression of IL-8 (a), TNF-a (b) and IL-18 (c) in AU in the liver. Data are presented as median with IQR. A: IL-8 tended to increase 5 hours after IA LPS administration. $p=0.09$ compared to control. $B$ : TNF-a mRNA levels tended to be increased 12 hours after IA LPS administration. $p=0.07$ compared to control. C: IL-18 mRNA levels were increased at 24 hours and 2 days after IA LPS administration. ${ }^{*} p=0.05$ and ${ }^{* *} p<0.005$ compared to control.

\section{Enterohepatic circulation alterations due to IA LPS exposure}

Gene expression of NTCP, the transporter responsible for BAs uptake from the portal circulation into the hepatocyte, was decreased in the liver of animals at 12 hours after IA LPS administration, compared to control ( $p<0.05$; Figure $4 a)$. In addition, gene expression of BSEP, the pump responsible for BAs excretion from the hepatocyte into the bile canaliculi for export into the gastrointestinal tract, was also decreased in the liver of animals at 12 hours after IA LPS administration ( $p<0.05$; Figure $4 b)$.

a

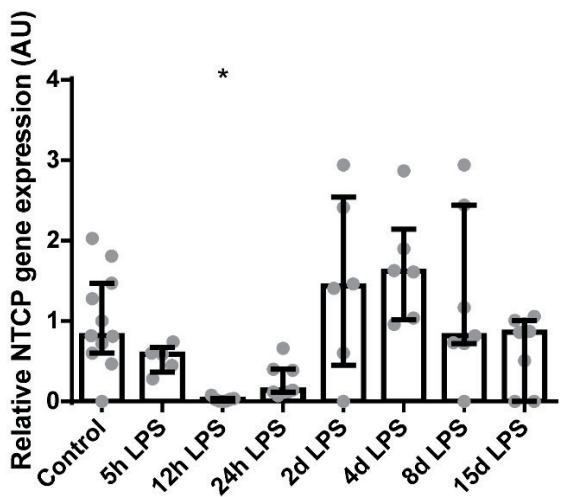

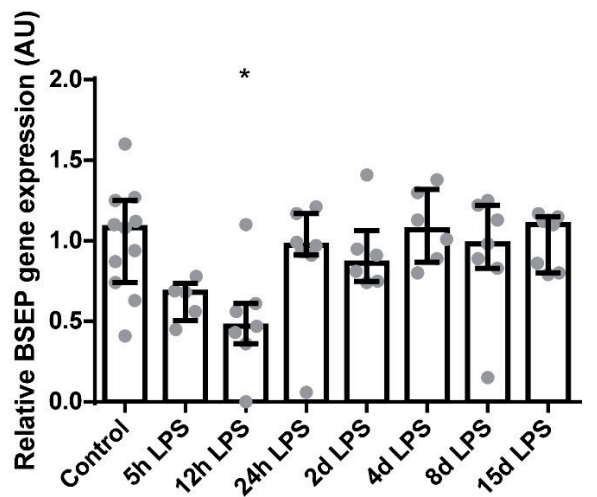

Figure 4. Relative hepatic NTCP and BSEP gene expression in AU. Data are presented as median with IQR. NTCP (a) and BSEP (b) were decreased 12 hours after IA LPS administration. ${ }^{*} p<0.05$ compared to control. 
In addition, plasma tBAs concentrations were increased in animals 12 hours after IA LPS administration, compared to control ( $p<0.05$; Figure 5).

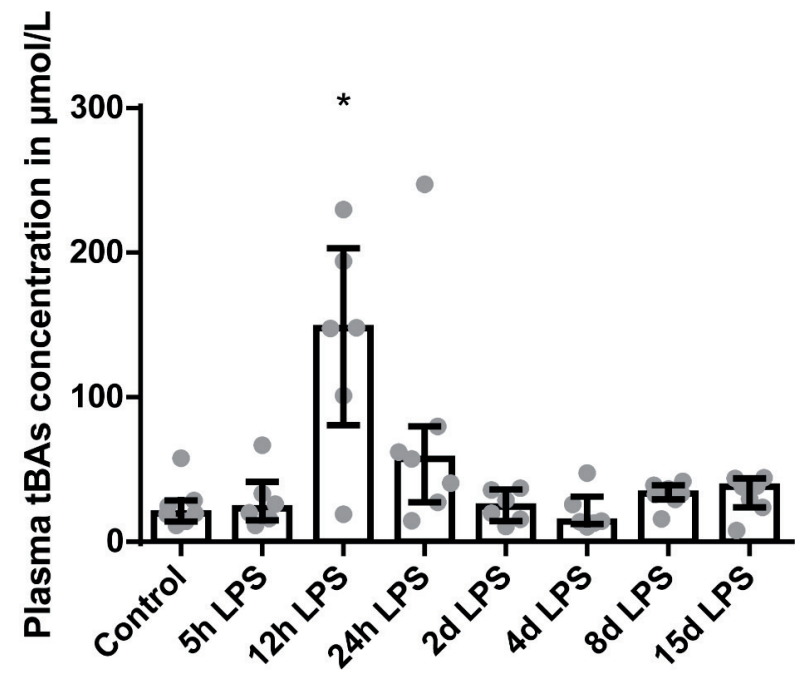

Figure 5. tBAs concentrations in plasma in $\mu \mathrm{mol} / \mathrm{L}$. Data are presented as median with IQR. Increased tBAs concentration in animals 12 hours after IA LPS administration. * $\mathrm{p}<0.05$ compared to control.

Furthermore, tBAs concentrations in liver and ileum homogenates did not differ between the groups (data not shown). Gene expression levels of CYP7A1 and CYP27A1, BA synthesis markers, did not differ among the groups (data not shown). 
Intestinal gene expression of ASBT, a transporter responsible for the uptake of BAs from the lumen into the enterocyte, was increased in animals 24 hours after LPS administration ( $p<0.05$; Figure 6 ) and tended to be increased 8 days after IA LPS administration, compared to control ( $p=0.07$; Figure 6).

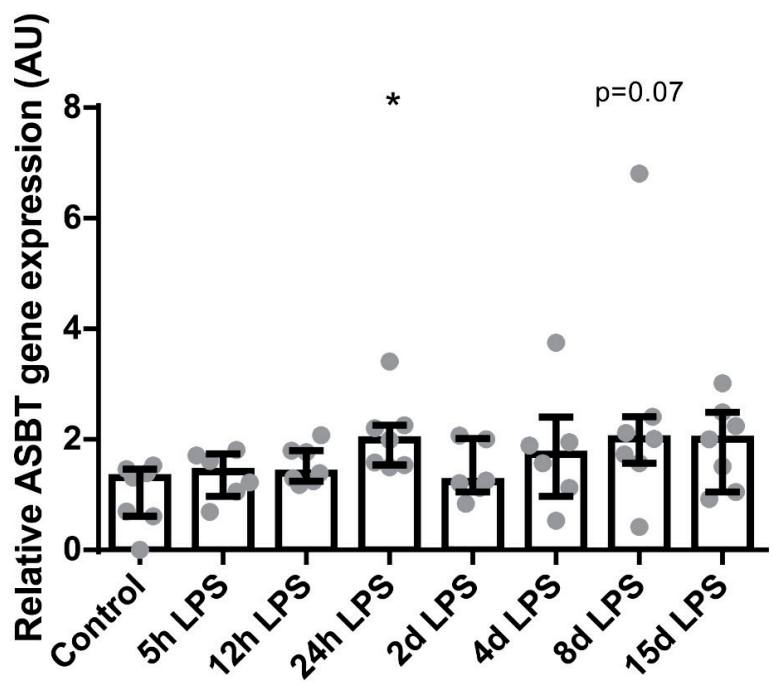

Figure 6. Relative gene expression of ASBT in AU in the terminal ileum. Data are presented as median with IQR. Increased ASBT expression in animals 24 hours and 8 days after IA LPS administration. * $p<0.05$ compared to control. $\mathrm{p}=0.07$ compared to control.

Furthermore, intestinal mRNA expression levels of FGF19 (a hormone regulating bile acid synthesis), IBABP (required for efficient apical to basolateral transport of conjugated BAs in ileal enterocytes) and OSTa- $\beta$ (transporters responsible for BAs excretion from the enterocyte into the portal circulation) did not differ between the groups (data not shown). An overview of the EHC changes is depicted in figure 7. 


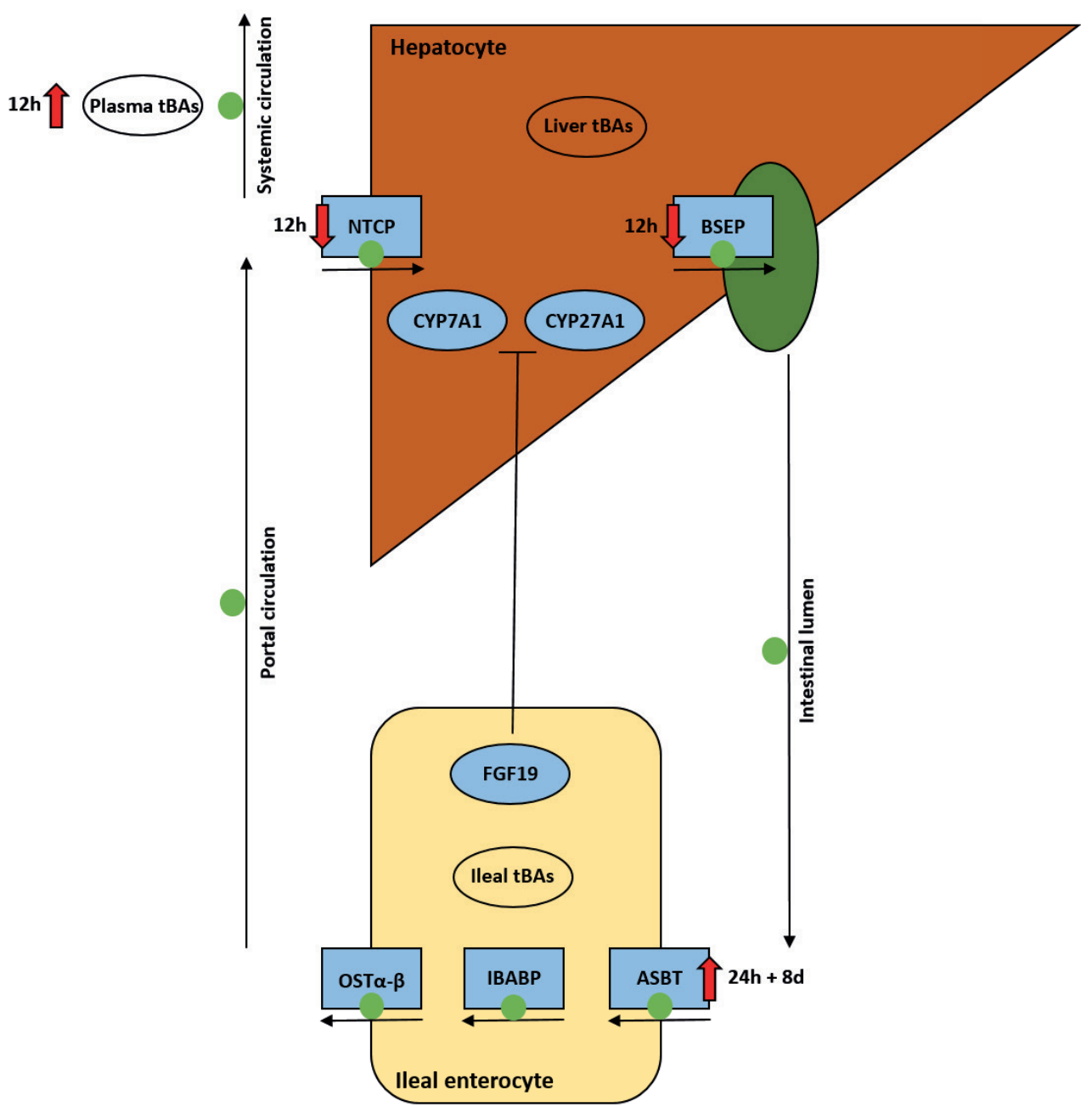

Figure 7. An overview of the enterohepatic circulation (EHC) of bile acids (BAs) and changes herein related to FIRS and liver inflammation. CYP7A1 and CYP27A1 play a role in BAs production, which can be inhibited by FGF19. Physiologically, BAs are transported from hepatocytes via BSEP, via the gallbladder subsequently into the intestinal lumen. Most BAs are reabsorbed in the terminal ileum by ASBT. Following enterocyte uptake, BAs bind to IBABP to traverse the cytoplasm of epithelial cells. BAs exit the basolateral site of the enterocyte via OSTa-OST $\beta$. Via the portal vein, BAs are transported back to the liver. NTCP takes up the BAs into the hepatocyte to be recycled. 
Decreased expression of NTCP and BSEP and resultant increased plasma BA levels are associated with FIRS and liver inflammation. Increased ASBT expression may also be inflammation dependent, or a compensatory mechanism to the postulated lower intraluminal BAs supply to maintain a constant BA pool in the EHC.

\section{Intestinal inflammation after IA LPS exposure}

Intestinal inflammation was detected by increased immunoreactivity of IBA1 (macrophages) in the terminal ileum of preterm lambs 2 days $(p<0.05$; Figure $8 b+d)$ and 4 days $(p=0.09$; Figure $8 c+d)$ after IA LPS administration, compared to control (Figure $8 a+d)$, which supports and extends earlier findings $(24,28)$.

A
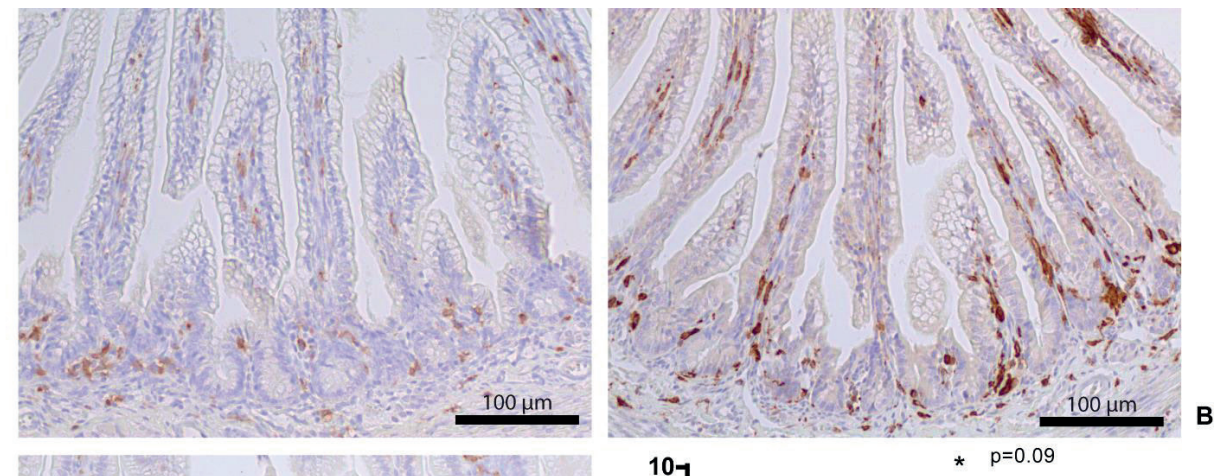

C
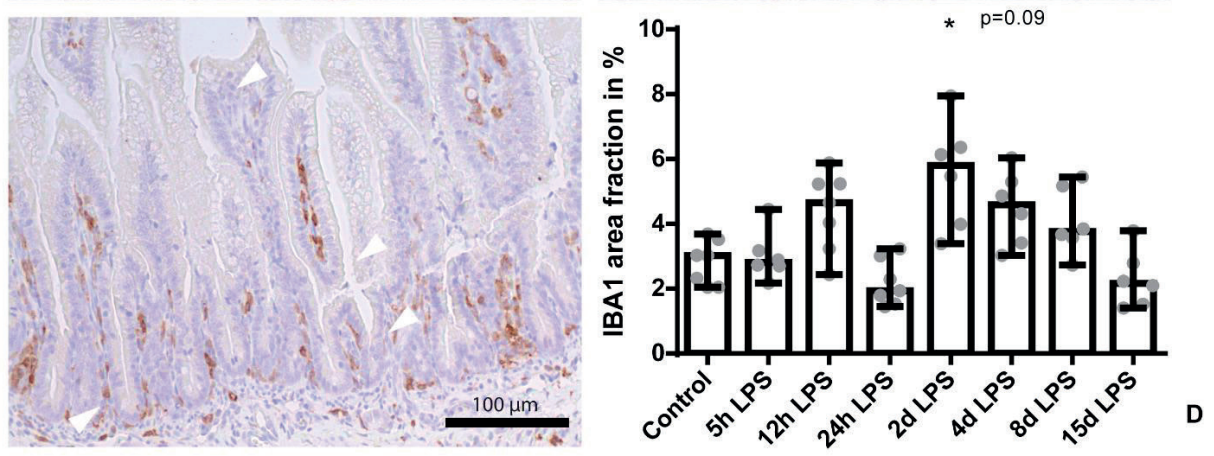

Figure 8. IBA1 immunoreactivity in the terminal ileum. Representative images of control (a), at day 2 after IA LPS administration (b) and at day 4 after LPS administration (c). Data are presented as medium with IQR (d). D: Increased IBA 1 positive surface area in animals 2 days and 4 days after IA LPS exposure. ${ }^{*} \mathrm{p}<0.05$ compared to control. $p=0.09$ compared to control. 
Moreover, ileal IL-18 mRNA levels tended to be increased 24 hours after IA LPS administration, compared to control ( $p=0.07$; Figure 9).

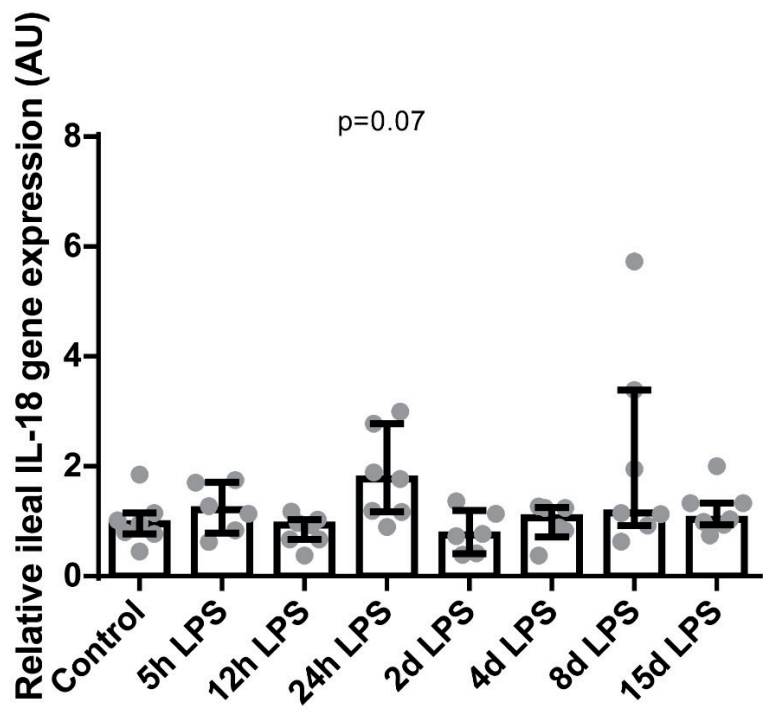

Figure 9. Relative gene expression of IL-18 in AU in the terminal ileum. Data are presented as median with IQR. IL-18 mRNA levels tended to be increased 24 hours after IA LPS administration. $p=0.07$ compared to control.

\section{Discussion}

In this study, we evaluated the time-dependent effects of intra-uterine administration of LPS from 15 days to 5 hours before premature delivery, on the liver and EHC of premature sheep.

Recent research showed that the fetal liver is an active immune organ with the ability of inducing an early and robust innate immune response activation, and immune activation is already initiated within 1 hour to 5 hours after an in utero inflammatory challenge (21). In our study, hepatic cytokine mRNA expression was increased from 5 hours until 2 days after IA LPS administration and these time points overlap with the ongoing fetal systemic immune response, characterized by increased circulatory IL-6 levels (5), suggesting that FIRS and local cytokine production in the liver are associated with each other. In accordance with these findings, chorioamnionitis-induced hepatic inflammation was associated with FIRS in a similar ovine model in which fetuses were assessed at day 2 and day 14 after IA LPS administration (19). Accordingly, in utero, the liver might thus be one of the first organs to induce an innate immune response by early cytokine production as a result of chorioamnionitis. 
An increased qualitative pathologic score of extramedullary hematopoiesis was observed in the liver parenchyma at 8 and 15 days after IA LPS administration. This increased score, reflecting clustered and conflated hepatic erythropoiesis in the parenchyma, is a hallmark of increased erythropoiesis in response to inflammation (26). Interestingly in human stillborns, a similar histological pattern was strongly associated with chorioamnionitis (29), suggesting that antenatal inflammation alters fetal extramedullary hematopoiesis. Although hepatic TNF-a, IL-8 and IL-18 levels have all been normalized at day 4 after IA LPS administration and no alterations in extramedullary hematopoiesis were observed until day 8 after IA LPS administration, it is possible that the early hepatic cytokine response contributed to the altered fetal erythropoiesis at day 8 and day 15 after IA LPS administration. In addition, fetal ileal inflammation was observed in the current and previous studies (24) with the most evident signs of inflammation 2 days and 4 days after IA LPS exposure. Transport of inflammatory mediators from the gut to the liver via the portal vein may therefore also contribute to hepatic inflammation, illustrated by extramedullary hematopoiesis. As seen in a previous study, it has been shown that upon infection and resultant immune responses, various hematopoietic factors including TLR ligands and cytokines promote extramedullary hematopoiesis in the liver (30). Therefore, the ongoing fetal systemic inflammatory response (5) or the direct exposure to inflammatory mediators through the transport from the gut via the portal vein, or a combination of both are likely the cause of the observed altered fetal extramedullary hematopoiesis in our study. Interestingly, increased production of hepatic cytokines was also found in neonatal rats with NEC and correlated with the progression of intestinal damage during disease development (18). Since increased hepatic TNF-a, IL-8 and IL-18 levels preceded and showed overlap with intestinal inflammation, it is likely that the early hepatic cytokine response contributed to intestinal inflammation. Whether hepatic cytokines contribute to intestinal inflammation in utero via the periphery or the bile remains to be elucidated (18).

The important role of the liver and the gut-liver axis in NEC pathogenesis is further emphasized by the fact that elevated ileal BAs and an altered expression of several BAs transporters can contribute to the development of NEC $(13-17,31)$. In our study, however, ileal BA levels were not changed. Only at 12 hours after IA LPS administration, plasma BA levels were increased, probably as a result of decreased NTCP expression. This indicates overflow of BAs returning via the portal circulation into the systemic circulation, with a rise in systemic BA levels as a result. Interestingly, cytokines are found to be key mediators in regulating hepatic expression of BA transporters during inflammation (32). Specifically, IL-6 can suppress NTCP and BSEP expression levels (10,32). The decreased expression of NTCP and BSEP in our study was thus most likely the result of the increased systemic IL-6 levels (5). Moreover, it was shown that LPS activates cytokine production of Kupffer cells, such as TNF-a, which in turn bind to their receptors on hepatocytes, resulting in reduced mRNA expression of several BAs transporters (10). Therefore, the increased hepatic TNF- $a$ expression, in our study, might also have contributed to the decreased mRNA expression of NTCP and BSEP $(10,15,33)$. This suggests that both FIRS and liver inflammation are causally related to the changes in the hepatic BA transporters. 
Reduced mRNA expression of various hepatic excretory BA transporters, e.g. BSEP, was observed in many animal models upon LPS treatment and also in patients with inflammation-induced cholestasis (10). The decreased NTCP expression might thus be a compensatory mechanism to protect the liver against damage during sepsis as BAs have been shown to be hepatotoxic during cholestasis (11). Our results probably show an earlier disease state in which LPS induced sepsis not yet has progressed to cholestasis since hepatic BA levels were unchanged, indicating that these animals were not cholestatic. Another compensatory mechanism might be the unchanged BAs synthesis, which in the normal situation would be expected to increase to remain a constant BA pool.

Intestinal ASBT mRNA expression was increased in animals 24 hours after IA LPS administration, which might be a response to the decreased transport of BAs from the liver to the gut 12 hours after IA LPS administration. No changes in BAs concentrations in the ileum were observed at any of the time points, suggesting that the increased ASBT expression may be a compensatory mechanism to the postulated lower intraluminal BAs supply to keep a constant BA pool in the EHC, as ASBT is known to play a major role in BA homeostasis (34). Moreover, it might also be related to the increased cytokines such as ileal TNF- $a$ and IL-18 levels, which in previous studies have been shown to upregulate ASBT expression $(35,36)$. Of note, intestinal BAs did not contribute to intestinal inflammation (24), as they were not increased.

The elevated BA levels in serum following IA LPS exposure were also measured in neonates with NEC in combination with depressed biliary BA levels, suggesting a failure of BA transport from the hepatocytes into the bile canaliculi (37). In addition, increased ASBT expression has been found in preterm infants and rodents with NEC $(14,16)$. However, in our study, initial alterations to the EHC normalized after IA LPS administration, suggesting that the duration of IA inflammation is important for the hepatic outcome. There is a defined window of vulnerability in which additional inflammatory hits, a premature born child is likely to encounter, might induce (additional) injury. This study highlights that the liver must be considered in neonatal care of preterm infants that suffered from inflammatory stress. Importantly, additional inflammation may have further impact on the liver and $\mathrm{EHC}$ and as a consequence the host in general. Based on our current findings, immune modulatory interventions such as nutrition and cytokine inhibitors might have the potential to improve neonatal wellbeing. In this context, timing of treatment initiation on the liver and $\mathrm{EHC}$ should be further studied.

A limitation of this study is the set-up in which the fixed moments of premature birth cannot exclude a potential influence of gestational age at start of IA infection. Furthermore, an unavoidable shortcoming of large animal studies is the relatively low number of animals per group.

In summary, in utero, the liver might be one of the first organs to induce an innate immune response by early cytokine production as a result of chorioamnionitis. An altered fetal erythropoiesis as a reaction to inflammation was detected at day 8 and day 15 after IA LPS administration that might be the result of hepatic cytokine production, ileal inflammation and ongoing FIRS. This ongoing fetal systemic inflammation and liver inflammation 
most likely also caused the changes in the decreased expression of several hepatic BAs transporters and resultant increased plasma BA levels that were observed 12 hours after IA LPS administration. Initial alterations to the EHC normalized over time, suggesting that the duration of IA inflammation is important for the hepatic outcome. Since a premature born child is likely to encounter additional postnatal inflammatory hits that might induce (additional) injury, this study highlights that the liver must be considered in neonatal care of preterm infants that suffered from inflammatory stress. 


\section{References}

1. Murphy SL, Mathews TJ, Martin JA, Minkovitz CS, Strobino DM. Annual Summary of Vital Statistics: 2013-2014. Pediatrics. 2017;139(6) DOI: 10.1542/peds.2016-3239.

2. Goldenberg RL, Hauth JC, Andrews WW. Intrauterine infection and preterm delivery. N Engl J Med. 2000;342(20):1500-7 DOI: 10.1056/NEJM200005183422007.

3. Galinsky R, Polglase GR, Hooper SB, Black MJ, Moss TJ. The consequences of chorioamnionitis: preterm birth and effects on development. J Pregnancy. 2013;2013:412831 DOI: 10.1155/2013/412831.

4. Kim CJ, Romero R, Chaemsaithong P, Chaiyasit N, Yoon BH, Kim YM. Acute chorioamnionitis and funisitis: definition, pathologic features, and clinical significance. Am J Obstet Gynecol. 2015;213(4 Suppl):S29-52 DOI: 10.1016/j.ajog.2015.08.040.

5. Gussenhoven R, Westerlaken RJJ, Ophelders D, Jobe AH, Kemp MW, Kallapur SG, Zimmermann LJ, Sangild PT, Pankratova S, Gressens P, Kramer BW, Fleiss B, Wolfs T. Chorioamnionitis, neuroinflammation, and injury: timing is key in the preterm ovine fetus. J Neuroinflammation. 2018;15(1):113 DOI: 10.1186/s12974-018-1149-x.

6. Andrews WW, Goldenberg RL, Faye-Petersen O, Cliver S, Goepfert AR, Hauth JC. The Alabama Preterm Birth study: polymorphonuclear and mononuclear cell placental infiltrations, other markers of inflammation, and outcomes in 23- to 32-week preterm newborn infants. Am J Obstet Gynecol. 2006;195(3):803-8 DOI: 10.1016/j.ajog.2006.06.083.

7. Gantert M, Been JV, Gavilanes AW, Garnier Y, Zimmermann LJ, Kramer BW. Chorioamnionitis: $a$ multiorgan disease of the fetus? J Perinatol. 2010;30 Suppl:S21-30 DOI: 10.1038/jp.2010.96.

8. Wolfs TG, Jellema RK, Turrisi G, Becucci E, Buonocore G, Kramer BW. Inflammation-induced immune suppression of the fetus: a potential link between chorioamnionitis and postnatal early onset sepsis. J Matern Fetal Neonatal Med. 2012;25 Suppl 1:8-11 DOI: 10.3109/14767058.2012.664447.

9. Been JV, Lievense S, Zimmermann LJ, Kramer BW, Wolfs TG. Chorioamnionitis as a risk factor for necrotizing enterocolitis: a systematic review and meta-analysis. J Pediatr. 2013;162(2):236-42 e2 DOI: 10.1016/j.jpeds.2012.07.012.

10. Kosters A, Karpen SJ. The role of inflammation in cholestasis: clinical and basic aspects. Semin Liver Dis. 2010;30(2):186-94 DOI: 10.1055/s-0030-1253227.

11. Perez MJ, Briz O. Bile-acid-induced cell injury and protection. World J Gastroenterol. 2009;15(14):1677-89 DOI: 10.3748/wjg.15.1677.

12. Hegyi P, Maleth J, Walters JR, Hofmann AF, Keely SJ. Guts and Gall: Bile Acids in Regulation of Intestinal Epithelial Function in Health and Disease. Physiol Rev. 2018;98(4):1983-2023 DOI: 10.1152/physrev.00054.2017.

13. Halpern MD, Dvorak B. Does abnormal bile acid metabolism contribute to NEC? Semin Perinatol. 2008;32(2):114-21 DOI: 10.1053/j.semperi.2008.01.005.

14. Halpern MD, Holubec H, Saunders TA, Dvorak K, Clark JA, Doelle SM, Ballatori N, Dvorak B. Bile acids induce ileal damage during experimental necrotizing enterocolitis. Gastroenterology. 2006;130(2):359-72 DOI: 10.1053/j.gastro.2005.10.023.

15. Cherrington NJ, Estrada TE, Frisk HA, Canet MJ, Hardwick RN, Dvorak B, Lux K, Halpern MD. The hepatic bile acid transporters Ntcp and Mrp2 are downregulated in experimental necrotizing enterocolitis. Am J Physiol Gastrointest Liver Physiol. 2013;304(1):G48-56 DOI: 10.1152/ ajpgi.00317.2012. 
16. Halpern MD, Weitkamp JH, Mount Patrick SK, Dobrenen HJ, Khailova L, Correa H, Dvorak B. Apical sodium-dependent bile acid transporter upregulation is associated with necrotizing enterocolitis. Am J Physiol Gastrointest Liver Physiol. 2010;299(3):G623-31 DOI: 10.1152/ajpgi.00242.2010.

17. Hulzebos CV, van Zoonen AG, Hulscher JB, Schat TE, Kooi EM, Koehorst M, Boverhof R, Krabbe PF, Groen AK, Verkade HJ. Fecal Bile Salts and the Development of Necrotizing Enterocolitis in Preterm Infants. PLoS One. 2017;12(1):e0168633 DOI: 10.1371/journal.pone.0168633.

18. Halpern MD, Holubec H, Dominguez JA, Meza YG, Williams CS, Ruth MC, McCuskey RS, Dvorak B. Hepatic inflammatory mediators contribute to intestinal damage in necrotizing enterocolitis. Am J Physiol Gastrointest Liver Physiol. 2003;284(4):G695-702 DOI: 10.1152/ajpgi.00353.2002.

19. Bieghs V, Vlassaks E, Custers A, van Gorp PJ, Gijbels MJ, Bast A, Bekers O, Zimmermann LJ, Lutjohann D, Voncken JW, Gavilanes AW, Kramer BW, Shiri-Sverdlov R. Chorioamnionitis induced hepatic inflammation and disturbed lipid metabolism in fetal sheep. Pediatr Res. 2010;68(6):46672 DOI: 10.1203/PDR.0b013e3181f70eeb.

20. Vlassaks E, Gavilanes AW, Bieghs V, Reinartz A, Gassler N, Van Gorp PJ, Gijbels MJ, Bekers O, Zimmermann LJ, Pillow JJ, Polglase GR, Nitsos I, Newnham JP, Kallapur SG, Jobe AH, ShiriSverdlov R, Kramer BW. Antenatal exposure to chorioamnionitis affects lipid metabolism in 7-week-old sheep. J Dev Orig Health Dis. 2012;3(2):103-10 DOI: 10.1017/S2040174412000049.

21. Zarate MA, Wesolowski SR, Nguyen LM, De Dios RK, Wilkening RB, Rozance PJ, Wright CJ. In utero inflammatory challenge induces an early activation of the hepatic innate immune response in late gestation fetal sheep. Innate Immun. 2020;26(7):549-64 DOI: 10.1177/1753425920928388.

22. Kuypers E, Wolfs TG, Collins JJ, Jellema RK, Newnham JP, Kemp MW, Kallapur SG, Jobe AH, Kramer BW. Intraamniotic lipopolysaccharide exposure changes cell populations and structure of the ovine fetal thymus. Reprod Sci. 2013;20(8):946-56 DOI: 10.1177/1933719112472742.

23. Kuypers E, Willems MG, Jellema RK, Kemp MW, Newnham JP, Delhaas T, Kallapur SG, Jobe AH, Wolfs TG, Kramer BW. Responses of the spleen to intraamniotic lipopolysaccharide exposure in fetal sheep. Pediatr Res. 2015;77(1-1):29-35 DOI: 10.1038/pr.2014.152.

24. Heymans $C$, de Lange IH, Lenaerts $K$, Kessels L, Hadfoune M, Rademakers G, Melotte V, Boesmans W, Kramer BW, Jobe AH, Saito M, Kemp MW, van Gemert WG, Wolfs T. Chorioamnionitis induces enteric nervous system injury: effects of timing and inflammation in the ovine fetus. Mol Med. 2020;26(1):82 DOI: 10.1186/s10020-020-00206-x.

25. Heymans C, Heij LR, Lenaerts K, den Dulk M, Hadfoune M, van Heugten C, Spiller OB, Beeton ML, Stock SJ, Jobe AH, Payne MS, Kemp MW, Kramer BW, Plat J, van Gemert WG, Wolfs T. Prophylactic Intra-Uterine beta-Cyclodextrin Administration during Intra-Uterine Ureaplasma parvum Infection Partly Prevents Liver Inflammation without Interfering with the Enterohepatic Circulation of the Fetal Sheep. Nutrients. 2020;12(5) DOI: 10.3390/nu12051312.

26. Paulson RF, Ruan B, Hao S, Chen Y. Stress Erythropoiesis is a Key Inflammatory Response. Cells. 2020;9(3) DOI: 10.3390/cells9030634.

27. Willems MG, Ophelders DR, Nikiforou M, Jellema RK, Butz A, Delhaas T, Kramer BW, Wolfs TG. Systemic interleukin-2 administration improves lung function and modulates chorioamnionitisinduced pulmonary inflammation in the ovine fetus. Am J Physiol Lung Cell Mol Physiol. 2016;310(1):L1-7 DOI: 10.1152/ajplung.00289.2015.

28. van Gorp C, de Lange IH, Massy KRI, Kessels L, Jobe AH, Cleutjens JPM, Kemp MW, Saito M, Usada H, Newnham J, Hütten M, Kramer BW, Zimmermann LJ, Wolfs TGAM. Intestinal Goblet Cell Loss during Chorioamnionitis in Fetal Lambs: Mechanistic Insights and Postnatal Implications. International Journal of Molecular Sciences. 2021;22(4):1946. 
29. Pfisterer C, Faber R, Horn LC. Chorioamnionitis-induced changes of fetal extramedullar hematopoiesis in the second trimester of gestation. Is diagnosis from fetal autopsy possible? Virchows Arch. 2005;446(2):150-6 DOI: 10.1007/s00428-004-1151-9.

30. Kim CH. Homeostatic and pathogenic extramedullary hematopoiesis. J Blood Med. 2010;1:13-9 DOI: $10.2147 / J B M . S 7224$.

31. Martin NA, Mount Patrick SK, Estrada TE, Frisk HA, Rogan DT, Dvorak B, Halpern MD. Active transport of bile acids decreases mucin 2 in neonatal ileum: implications for development of necrotizing enterocolitis. PLoS One. 2011;6(12):e27191 DOI: 10.1371/journal.pone.0027191.

32. Hartmann G, Cheung AK, Piquette-Miller M. Inflammatory cytokines, but not bile acids, regulate expression of murine hepatic anion transporters in endotoxemia. J Pharmacol Exp Ther. 2002;303(1):273-81 DOI: 10.1124/jpet.102.039404.

33. Cherrington NJ, Slitt AL, Li N, Klaassen CD. Lipopolysaccharide-mediated regulation of hepatic transporter mRNA levels in rats. Drug Metab Dispos. 2004;32(7):734-41 DOI: 10.1124/ dmd.32.7.734.

34. Ferrebee CB, Dawson PA. Metabolic effects of intestinal absorption and enterohepatic cycling of bile acids. Acta Pharm Sin B. 2015;5(2):129-34 DOI: 10.1016/j.apsb.2015.01.001.

35. Dobrenen H, Patrick SM, Khailová L, DvpYák B, Halpern M. T1877 Interleukin-18 Upregulates the Apical Sodium-Dependent Bile Acid Transporter (ASBT) in Neonatal Rat lleum and IEC-6 Cells. Gastroenterology. 2010;138.

36. Rogan DT. Overexpression of the Apical Sodium-Dependent Bile Acid Transporter to Replicate Necrotizing Enterocolitis in IEC-6 Cells. The University of Arizona.; 2012.

37. Moss RL, Das JB, Raffensperger JG. Necrotizing enterocolitis and total parenteral nutritionassociated cholestasis. Nutrition. 1996;12(5):340-3 DOI: 10.1016/s0899-9007(96)00062-7. 



\section{Prophylactic intra-uterine}

$\beta$-cyclodextrin administration during intra-uterine Ureaplasma parvum infection partly prevents liver inflammation without interfering with the enterohepatic circulation of the fetal sheep

C. Heymans, L.R. Heij, K. Lenaerts, M. den Dulk, M. Hadfoune, C. van Heugten, O.B. Spiller, M.L. Beeton, S.J. Stock, A.H. Jobe, M.S. Payne, M.W. Kemp, B.W. Kramer, J. Plat, W.G. van Gemert, T.G.A.M. Wolfs 


\section{Abstract}

\section{Background}

Chorioamnionitis can lead to inflammation and injury of the liver and gut, thereby predisposing patients to adverse outcomes such as necrotizing enterocolitis (NEC). In addition, intestinal bile acids (BAs) accumulation is causally linked to NEC development. Plant sterols are a promising intervention to prevent NEC development, considering their anti-inflammatory properties in the liver. Therefore, we investigated whether an intraamniotic (IA) Ureaplasma parvum (UP) infection affected the liver and enterohepatic circulation (EHC) and evaluated whether an IA administered plant sterol mixture dissolved in $\beta$-cyclodextrin exerted prophylactic effects.

\section{Materials and Methods}

An ovine chorioamnionitis model was used in which liver inflammation and the EHC were assessed following IA UP exposure in the presence or absence of IA prophylactic plant sterols (a mixture of $\beta$-sitosterol and campesterol dissolved in $\beta$-cyclodextrin (carrier)) or carrier alone.

\section{Results}

IA UP exposure caused an inflammatory reaction in the liver, histologically seen as clustered and conflated hepatic erythropoiesis in the parenchyma, which was partially prevented by IA administration of sterol $+\beta$-cyclodextrin or $\beta$-cyclodextrin alone. In addition, IA administration of $\beta$-cyclodextrin prior to UP caused changes in the expression of several hepatic BAs transporters, without causing alterations in other aspects of the EHC.

\section{Conclusion}

IA UP exposure caused an inflammatory reaction in the liver, which was partially prevented by IA administration of sterol $+\beta$-cyclodextrin or $\beta$-cyclodextrin alone. In addition, IA administration of $\beta$-cyclodextrin prior to UP caused changes in the expression of several hepatic BAs transporters, without causing alterations in other aspects of the EHC. Thereby, the addition of plant sterols to the carrier $\beta$-cyclodextrin did not have additional effects. 


\section{Introduction}

Preterm birth, birth before 37 weeks of gestation, is the leading cause of morbidity and mortality among infants worldwide (1). An important cause of preterm birth is chorioamnionitis, an inflammatory cell infiltration of the fetal membranes, which is defined as an independent risk factor for the development of necrotizing enterocolitis (NEC) (2-5). The most common organism isolated from the amniotic fluid of pregnant women with chorioamnionitis is Ureaplasma parvum (UP), a commensal of the urogenital tract of humans (6-8). UP colonization in preterm infants has been associated with an increased incidence of NEC (2-5).

A strong interaction exists between the intestine and liver, wherein a crucial role is played by the enterohepatic circulation (EHC) of bile acids (BAs). There is increasing attention towards the role of BAs as critical regulators of intestinal epithelial function (9). Within this context, their contribution to NEC pathophysiology has recently been studied. In the week preceding NEC manifestation, fecal unconjugated BA levels were found to be higher in preterm infants, eventually developing NEC compared with gestation-matched controls (10). More precisely, the intraluminal accumulation of conjugated BAs resulted in intestinal epithelial damage, similar to the histopathological findings in NEC (11). These high concentrations of intraluminal BAs might be the result of increased BA synthesis, as was shown in a rat NEC model (12). In addition, the increased expression of the apical sodium-dependent bile acid transporter (ASBT) in the terminal ileum, a protein involved in the uptake of conjugated BAs into the enterocytes, was reported in preterm infants with NEC and in an experimental NEC model with rodents which correlated with the location of intestinal damage, suggesting increased BAs uptake by enterocytes $(13,14)$. Consequently, high concentrations of intraluminal BAs resulted in their accumulation within enterocytes, with concomitant enterocyte damage (10). Moreover, a decreased expression of the ileal bile acid-binding protein (IBABP) was seen in the terminal ileum of rats in an experimental NEC model, suggesting insufficient transport from the apical to the basolateral side of enterocytes with consequent $B A s$ accumulation within the enterocytes (14). The important role of the liver and the gut-liver axis in NEC pathogenesis is further underlined by the presence of increased hepatic inflammation in neonatal rats with NEC, which correlated with the progression of intestinal damage during disease development (15).

Interestingly, in a recent study, chorioamnionitis induced by six days of intra-uterine UP exposure caused a reduced amount of conjugated BAs in the enterocytes of the terminal ileum of fetal sheep (16). In addition, it was shown that fetuses exposed to endotoxininduced chorioamnionitis develop hepatic inflammation and a disturbed lipid metabolism in utero (17). These findings prompted us to investigate the liver and EHC alterations in a model of UP-induced chorioamnionitis in fetal sheep.

From a treatment perspective, plant sterols, which are dietary constituents present in vegetable oils, nuts, grains and fruit (18), seem promising due to their immune-modulatory properties (19-27). Moreover, they have even shown to be anti-inflammatory in the liver of mice that developed non-alcoholic fatty liver disease (NASH) (28). We previously showed 
that fetal gut inflammation and mucosal damage following IA UP exposure were prevented by prophylactic plant sterol supplementation in the amniotic fluid (16). Therefore, the second aim of this study was to investigate the effects of prophylactic IA administration of a plant sterol mixture dissolved in $\beta$-cyclodextrin as carrier on liver inflammation and potential EHC alterations.

\section{Materials and Methods}

\section{Animal model and experimental procedures}

The animal experiments were approved by the Animal Ethics Committee of the University of Western Australia (Perth, Australia; ethical approval code: RA/3/100/1378). The animal model and experimental procedures were previously described (16). In short, fifty ewes with singleton fetuses were randomly assigned to the six different study groups (figure 1). After drop-outs, the group size for data analyses was six to seven animals per group. One group of the date-mated pregnant ewes received an IA ultrasound-guided injection of Ureaplasma parvum serovar 3 (strain HPA5, $10^{7}$ color-changing units [CCU]), six days prior to delivery. To evaluate the preventive effect of plant sterols, the amniotic fluid in two experimental groups was enriched with a mixture of $\beta$-sitosterol $(70 \%)$ and campesterol (30\%) (total of $0.6 \mathrm{mg} / \mathrm{ml}$ ), dissolved in a carrier, 18\% 2-hydroxypropyl- $\beta$-cyclodextrin (H107, Sigma Aldrich, St. Louis, MO, USA) eleven days prior to delivery in combination with saline, or UP six days prior to delivery. To assess the carrier effect separately from the plant sterols, in two groups, the carrier was given alone with saline or carrier in combination with UP. A group receiving IA injections of saline (eleven or six days prior to delivery) served as control (figure 1).

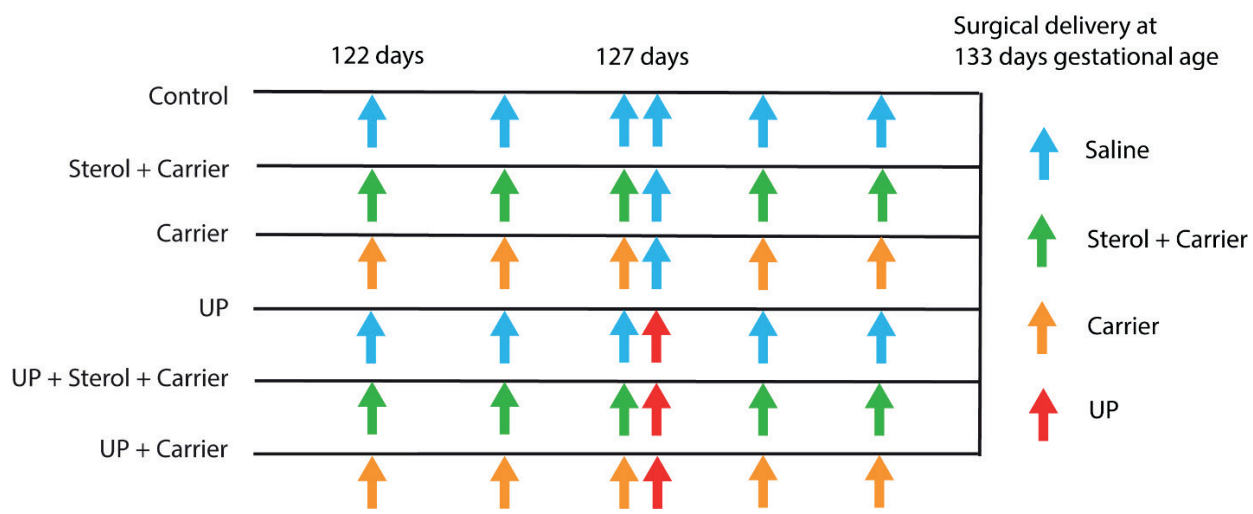

Figure 1. Experimental design. Animals were randomly assigned to six different study groups; control: $n=6$, plant sterols in carrier: $n=6$, carrier alone: $n=6$, Ureaplasma Parvum (UP): $n=7$, UP + plant sterols in carrier: $n=7$, UP + Carrier alone: $n=7$. Plant sterols dissolved in the carrier ( $\beta$-cyclodextrin) were administered by an intraamniotic (IA) injection at $122 \mathrm{~d}$ of gestational age (GA), before onset of chorioamnionitis and were repeated every two days until 131d GA. The animals were prematurely delivered at 133d GA. Two groups received the carrier alone (without added plant sterols) IA to assess the effects of the carrier separately from the plant sterols. Ureaplasma parvum serovar 3 ( $10^{7}$ color-changing units [CCUs]) was given by IA injection at $127 \mathrm{~d}$ GA to induce chorioamnionitis. The control group received saline on all administration time points. 
Fetuses were delivered preterm by cesarean section at 133 days of gestational age (term ewe gestation $=150$ days), the equivalent of 33-34 weeks of human gestation. After cesarean section, fetuses were euthanized directly with intravenous pentobarbitone (100 $\mathrm{mg} / \mathrm{kg}$, Valabarb, Jurox, Rutherford, NSW, Australia). During necropsy, blood, liver, and terminal ileum samples were sampled.

\section{Qualitative analysis of liver histology}

H\&E slides were scored on a zero to four scale for degree of hepatic sinusoidal dilatation, shape, and size of central veins, and number and location of extramedullary hematopoietic clusters by an independent pathologist blinded to the experimental setup. Zero represented no sinusoidal dilatation, no divergent shape or size of central veins, and no inflammation. Four represented the other end of the scale, namely pronounced sinusoidal dilatation throughout the parenchyma, large central veins with venous stowing throughout the parenchyma, and a severely increased number of clusters of hematopoiesis.

\section{Total bile acid assay}

Total bile acids (tBAs) in plasma and liver homogenate were measured by an enzymatic cycling method using the Total Bile Acids Assay kit, according to the manufacture protocol (Diazyme Laboratories, Poway, CA, USA). tBAs in liver homogenate were corrected for protein content.

\section{RNA extraction and real-time PCR}

RNA was isolated from snap frozen liver and ileum tissue using TRI reagent (Thermo Fisher Scientific, Waltham, MA, USA)/chloroform extraction. The RNA was reverse transcribed into CDNA using a sensifast cDNA Synthesis kit (Bioline, London, UK). Quantitative realtime PCR (qPCR) was executed with the selected primer in Sensimix SYBR \& Fluorescein Kit (Bioline, London, UK). The qPCR reactions were performed with the use of the LightCycler 480 Instrument (Roche Applied Science, Basel, Switzerland) for 45 cycles. The gene expression levels of cholesterol 7 alpha-hydroxylase (CYP7A1), Cytochrome P450 Family 27 Subfamily A Member 1 (CYP27A1), $\mathrm{Na}^{+}$-taurocholate cotransporting polypeptide (NTCP), bile salt export pump (BSEP), apical sodium-dependent bile acid transporter (ASBT), fibroblast growth factor 19 (FGF19), ileal bile acid-binding protein (IBABP) and organic solute transporter alpha-beta (OSTa- $\beta$ ) were determined to assess changes in the EHC. LinRegPCR software (version 2016.0, Heart Failure Research Center, Academic Medical Center, Amsterdam, the Netherlands) was used to calculate the expression levels. As a normalization factor, the geometric mean of the expression levels of three reference genes (ribosomal protein S15 (RPS15), glyceraldehyde 3-phosphate dehydrogenase (GAPDH) and peptidylprolyl isomerase A (PPIA)) was calculated. The data were expressed as fold increase over the control value, arbitrary unit (AU). An overview of the used primer sequences is shown in Table 1. 
Table 1. Primer sequences

\begin{tabular}{|c|c|c|}
\hline Primer & Forward & Reverse \\
\hline RPS15 & 5'-CGAGATGGTGGGCAGCAT-3' & 5'-GCTTGATTTCCACCTGGTTGA-3' \\
\hline GAPDH & 5'-GGAAGCTCACTGGCATGGC-3' & 5'-CCTGCTTCACCACCTTCTTGG-3' \\
\hline PPIA & 5'-TTATAAAGGTTCCTGCTTTCACAGAA-3' & 5'-ATGGACTTGCCACCAGTACCA-3' \\
\hline CYP7A1 & 5'-GGGCATCACAAGCAAACACC-3' & 5'-GATGATACTGTCTAGCACGGG-3' \\
\hline CYP27A1 & 5'- CCCAAGAATACCCAGTTTGTGC-3' & 5'- GGTGGCAGAAGACTCAGTTCA-3' \\
\hline NTCP & 5'-TCCTCAAATCCAAACGGCCA-3' & 5'-GTTTGGATCGTCCATTGAGGC-3' \\
\hline BSEP & 5'-ACTCAGTAATTCTTCGCAGTGTG-3' & 5'-ATCGAAACAATCGAAAGAAGCCA-3' \\
\hline ASBT & 5'-CATGGACCTGAGCGTCAGCAT-3' & 5'-CACGGAGACGGGAACAACAA-3' \\
\hline FGF19 & 5'-TTGATGGAGATCAGGGCGGT-3' & 5'-CGGATCTCCTCCTCGAAAGC-3' \\
\hline IBABP & 5'-ACAAGAAGTTCAAGGTCACCG-3' & 5'-TGATACGGCTTTATGGCCCC-3' \\
\hline OSTa & 5'-ATCCCAGGTACACGGCAGAT-3' & 5'-ATTGAGGCCAGGACAAGCAA-3' \\
\hline OST $\beta$ & $5^{\prime}-\mathrm{CCGAGTAGAGGATGCAACTCC-} 3^{\prime}$ & 5'-TTTGTTTTTCCGGTGGCAGC-3' \\
\hline
\end{tabular}

\section{Data analysis}

Statistical analyses were performed using GraphPad Prism (version 6.01, GraphPad Software Inc., La Jolla, CA, USA). Data are presented as median with interquartile range (IQR). Differences in the qualitative analysis of liver histology using scorings were assessed using the Kruskal-Wallis test followed by Dunn's post hoc test. A square root transformation was applied to the other data to obtain normal distribution. A comparison between different experimental groups was performed using the two-way ANOVA followed by Tukey's or Sidak's multiple comparisons test. Differences were considered statistically significant at $\mathrm{p}<0.05$.

\section{Results}

\section{Sterol + carrier, as well as carrier alone, partly decrease hepatic inflammation due to UP-induced chorioamnionitis}

Histologically, no signs of sinusoidal dilatation, divergent shape or size of central veins, or inflammation were observed in the control group and animals exposed to the sterol + carrier or carrier alone (figure $2 \mathrm{E}, 2 \mathrm{~F}$ and $2 \mathrm{G}$ ). All animals exposed to UP (all groups) displayed pronounced sinusoidal dilation throughout the entire liver parenchyma (all $\mathrm{p}<0.01$; figure $2 \mathrm{E}$ ) without dilatation of the central veins. Administration of sterol + carrier or carrier alone prior to UP exposure did not decrease sinusoidal dilation. However, the animals treated with sterol + carrier prior to UP displayed enlarged central veins compared to control, sterol + carrier, UP, and UP + carrier (all $p<0.10$; figure $2 \mathrm{~F}$ ), without shape divergence.

A significant increase in the number of erythropoietic clusters was observed in animals exposed to UP ( $p<0.005$; figure $2 \mathrm{G}$ ), as the extramedullary hematopoiesis is interpreted as clustered and conflated hepatic erythropoiesis in the parenchyma (figure 2B, 2C and 2D). The administration of sterol + carrier or carrier alone prior to UP exposure tended to have lower numbers of erythropoietic clusters (both $p<0.10$; figure $2 \mathrm{G}$ ), suggesting that the 
carrier exerted this anti-inflammatory effect while dissolving sterols in the carrier did not have an additional effect on top of the carrier.

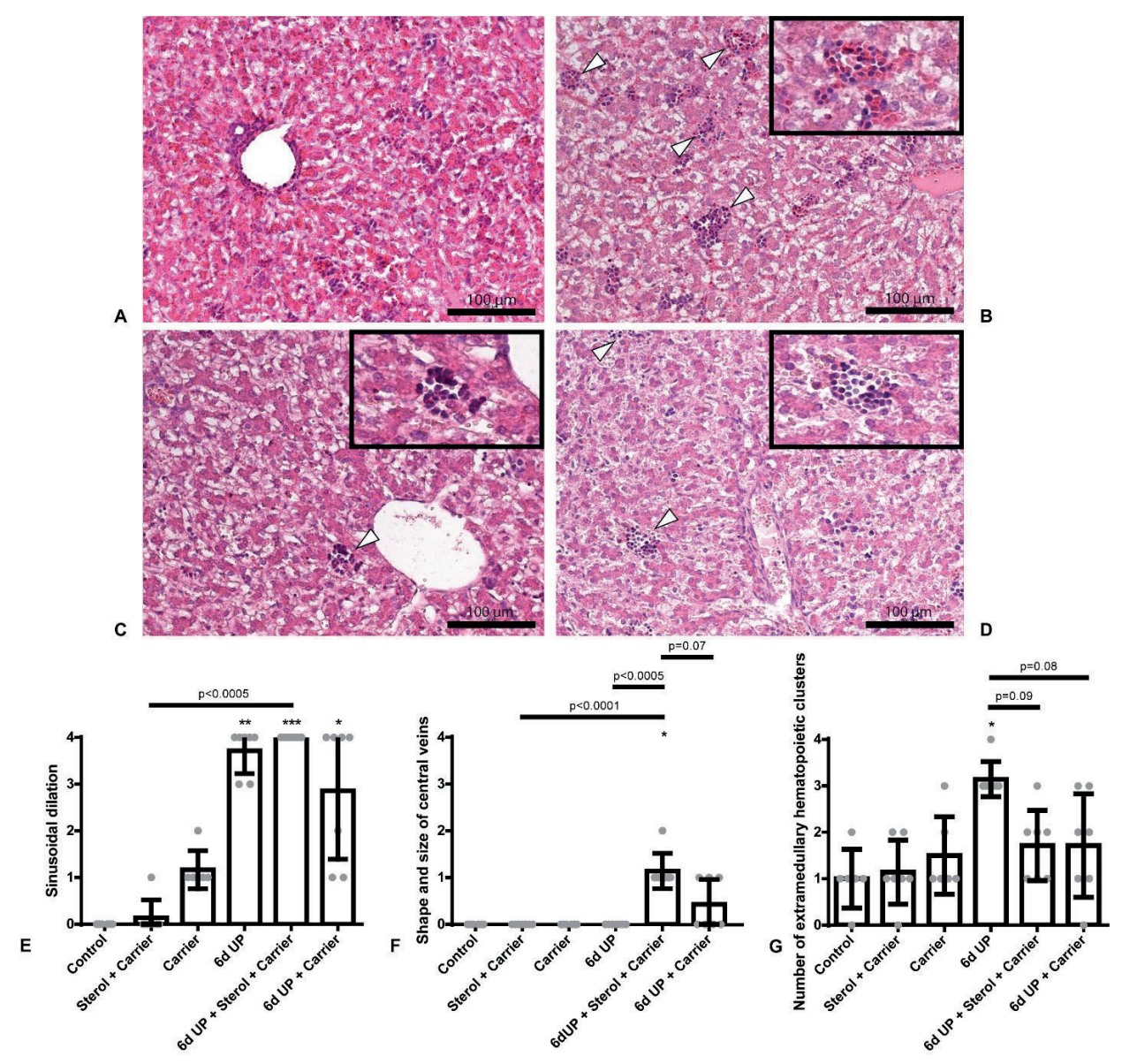

Figure 2. H\&E slides scored on a zero to four scale for degree of sinusoidal dilatation, shape and size of central veins, and number and location of extramedullary hematopoietic clusters by an independent pathologist. Representative images of the control (A), UP (B), UP + sterols dissolved in carrier (C) and UP + carrier alone (D). E: Increased sinusoidal dilation in animals exposed to UP, UP + sterols dissolved in the carrier, and UP + carrier alone. ${ }^{*} p<0.01,{ }^{* *} p<001,{ }^{* * *} p<0.0005$ compared to control. F: The UP + sterol dissolved in the carrier group displayed enlarged central veins, without shape divergence. ${ }^{*} p=0.0002$ compared to control. G: Increased number of extramedullary hematopoietic clusters in the animals exposed to UP. Treatment with prophylactic IA sterols dissolved in the carrier or carrier alone prior to UP exposure tended to decrease the number of extramedullary hematopoietic clusters. Specifically, the extramedullary hematopoiesis in all the animals exposed to UP is interpreted as clustered and conflated hepatic erythropoiesis in the parenchyma, which are indicated by white triangles. ${ }^{*} \mathrm{p}=0.001$ compared to control. 


\section{Administration of the carrier alone prior to UP exposure causes increased BSEP} expression in the liver

NTCP and BSEP mRNA expression levels were not altered in animals exposed to UP alone, compared to control (figure $3 \mathrm{~A}$ and $3 \mathrm{~B}$ ). However, in the UP + carrier group, an increased BSEP mRNA expression was observed compared with UP alone or UP + sterol + carrier $(p<0.05$; figure 3B).

A
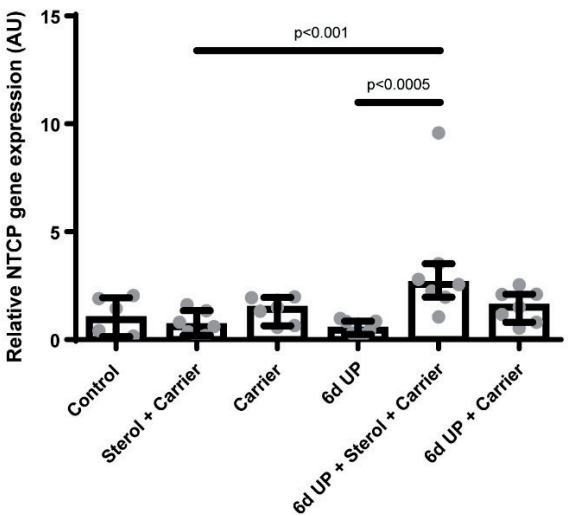

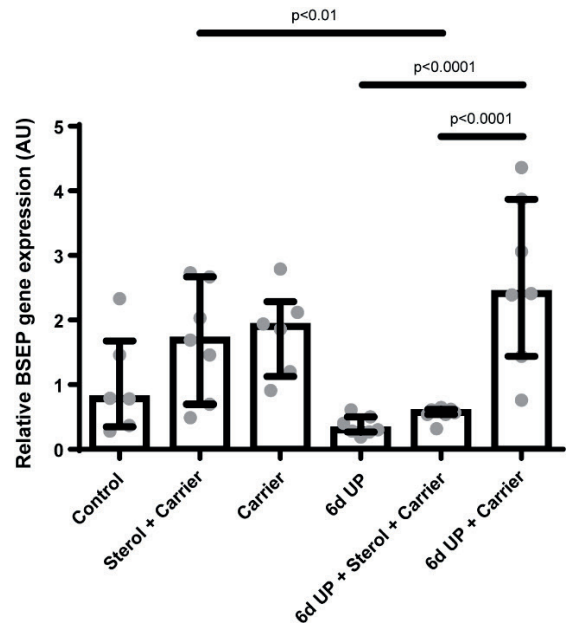

Figure 3. Relative gene expression of Na+-taurocholate cotransporting polypeptide (NTCP) (A) and bile salt export pump (BSEP) (B) in AU in the liver. A: Increased NTCP gene expression in animals exposed to UP + sterols dissolved in the carrier. B: Increased BSEP gene expression in animals exposed to UP + carrier alone.

No differences in the amount of tBAs in plasma or the liver were measured (data not shown). Furthermore, no differences were observed in the mRNA expression of the BAs synthesis markers CYP7A1 and CYP27A1 (data not shown). In addition, no differences were observed in the mRNA expression of any of the BAs transporters in the gut (ASBT, OSTa- $\beta$; data not shown), as well as intestinal FGF19 or IBABP (data not shown). 


\section{Discussion}

Chorioamnionitis induced by IA UP exposure for six days caused an inflammatory reaction in the liver, characterized by increased sinusoidal dilation and an increased number of extramedullary hematopoietic clusters. Specifically, clustering and conflation of hepatic erythropoiesis in the parenchyma was observed in these animals. This phenomenon was also observed in human babies born stillborn, in whom there was an increase in total hematopoiesis and erythropoiesis with clustering of erythropoietic cells, which was strongly associated with chorioamnionitis (29), implicating that antenatal inflammation alters fetal extramedullary hematopoiesis. The observed fetal hepatic cellular response to chorioamnionitis in our study might be the result of the fetal systemic inflammatory response (FIRS; increased circulatory IL-6 levels (16)), or direct exposure to inflammatory mediators through the transport from the gut via the portal vein, or a combination of both. Additionally, the distribution of erythropoiesis was altered in the previous mentioned study in humans, in which erythropoietic activity was often expanded over the whole portal field and the sinusoids in the case of chorioamnionitis (29). However, in our study, we observed alterations of fetal intrahepatic erythropoiesis in the parenchyma. Whether this difference is species-dependent remains to be elucidated, since no previous data on alterations of fetal intrahepatic erythropoiesis is present in sheep.

In our study, prophylactic IA exposure to carrier or carrier enriched with plant sterols prior to IA UP exposure partly decreased the number of clusters of erythropoiesis in the liver parenchyma. In our study, the carrier $\beta$-cyclodextrin exerted similar anti-inflammatory effects in the liver as was previously observed in the gut by plant sterols (16). The effects in the gut were explained by the plant sterols and the carrier $\beta$-cyclodextrin apparently having overlapping working mechanisms (16). Given the fact that plant sterols dissolved in the carrier, and the carrier alone possessed similar anti-inflammatory effects, our data suggests that the anti-inflammatory effects in the liver might be solely the result of the carrier $\beta$-cyclodextrin.

Caution to use plant sterols in the fetal setting of chorioamnionitis might be required, since sterol-treated animals displayed enlarged central veins in combination with pronounced sinusoidal dilatation, implying hepatic congestion (30). However, not significant, in the case of carrier administration alone these effects were also visible, concluding that one should be cautious here as well.

A previous study in the same model showed that chorioamnionitis induced by six days of IA UP exposure caused a reduction of conjugated BAs in the enterocytes of the terminal ileum of fetal sheep in the same model (16). This might indicate that, as a result of chorioamnionitis, the uptake of BAs from the lumen into the enterocyte is reduced, or the amount of $B A s$ in the lumen is reduced by a potential decreased excretion of BAs from the liver into the intestine, or a reduced BA synthesis. However, in our study, none of the above could explain the reduction of conjugated BAs in the enterocytes of the terminal ileum, since mRNA expression levels of the BA transporter responsible for the uptake of conjugated BAs in the terminal ileum, ASBT, were unchanged in animals exposed to UP. Moreover, mRNA expression levels of the pump responsible for the excretion of BAs from 
the hepatocytes into the bile canaliculi, BSEP, remained unaltered in animals exposed to UP alone.

Our findings suggest that the BAs synthesis in the liver of UP-exposed animals remained unchanged. This is an interesting finding since we previously demonstrated in these animals a reduction of conjugated BAs in enterocytes, and reduced fetal circulatory lathosterol concentrations, indicative of a reduced (hepatic) endogenous cholesterol synthesis (16). Since BAs are synthesized via oxidation of cholesterol (31), we assumed we would detect an altered syntheses of primary BAs as a result of IA UP exposure. However, the liver cholesterol pool was sufficient to such an extend that it did not have a negative impact on the syntheses of primary BAs. It might be possible that the conjugated BAs that were not absorbed in the terminal ileum of the fetal sheep via the ASBT were deconjugated in the colon and subsequently passively absorbed. When the deconjugated BAs would not have been passively absorbed in the colon, they would be lost in the feces. In this case, one would have expected increased BAs syntheses to maintain a constant BA pool, suggesting that there was no loss of BAs in our study.

In our study, we could not observe the EHC alterations found in experimental NEC and neonates with NEC. A possible explanation for this discrepancy could be that the EHC alterations contributing to NEC pathology do not have their origin in utero but might manifest after birth.

Finally, sterols dissolved in the carrier or carrier alone did not cause any alterations in the EHC. However, in combination with UP, increased mRNA expression levels of NTCP and BSEP were found. Although exposure to plant sterols dissolved in the carrier as well as the $\beta$-cyclodextrin carrier alone prior to UP caused changes in the expression of several hepatic BAs transporters, this did not cause EHC alterations further up in the circulation, e.g., altered BAs synthesis or altered ASBT mRNA expression levels, suggesting that these alterations might not be biologically relevant.

Overall we can conclude from this study that the observed effects are not the result of the addition of plant sterols to the carrier $\beta$-cyclodextrin but can be fully assigned to the carrier $\beta$-cyclodextrin alone. We have previously found that plant sterols and the carrier $\beta$-cyclodextrin protect the fetal gut against chorioamnionitis induced gut inflammation and injury (16). Based on these earlier findings and the current study, we conclude that $\beta$-cyclodextrin is an interesting pharmaceutical target to protect the gut and liver against the negative consequences of perinatal inflammatory stress $(32,33)$. Importantly, cyclodextrins are highly water soluble, "ready-made", and commercially available. Its pharmacokinetics and the optimal moment and route of administration in the fetal or neonatal context warrant further investigation.

In summary, IA UP exposure caused an inflammatory reaction in the liver, which was partially prevented by IA $\beta$-cyclodextrin administration. In addition, IA administration of $\beta$-cyclodextrin prior to UP caused changes in the expression of several hepatic BAs transporters, without causing alterations in other aspects of the EHC. Thereby, the addition of plant sterols to the carrier $\beta$-cyclodextrin did not have additional effects. 


\section{References}

1. Murphy SL, Mathews TJ, Martin JA, Minkovitz CS, Strobino DM. Annual Summary of Vital Statistics: 2013-2014. Pediatrics. 2017;139(6) DOI: 10.1542/peds.2016-3239.

2. Silwedel C, Speer CP, Glaser K.Ureaplasma-associated prenatal, perinatal, and neonatalmorbidities. Expert Rev Clin Immunol. 2017;13(11):1073-87 DOI: 10.1080/1744666X.2017.1381559.

3. Been JV, Lievense S, Zimmermann LJ, Kramer BW, Wolfs TG. Chorioamnionitis as a risk factor for necrotizing enterocolitis: a systematic review and meta-analysis. J Pediatr. 2013;162(2):236-42 e2 DOI: 10.1016/j.jpeds.2012.07.012.

4. Andrews WW, Goldenberg RL, Faye-Petersen O, Cliver S, Goepfert AR, Hauth JC. The Alabama Preterm Birth study: polymorphonuclear and mononuclear cell placental infiltrations, other markers of inflammation, and outcomes in 23- to 32-week preterm newborn infants. Am J Obstet Gynecol. 2006;195(3):803-8 DOI: 10.1016/j.ajog.2006.06.083.

5. Okogbule-Wonodi AC, Gross GW, Sun CC, Agthe AG, Xiao L, Waites KB, Viscardi RM. Necrotizing enterocolitis is associated with ureaplasma colonization in preterm infants. Pediatr Res. 2011;69(5 Pt 1):442-7 DOI: 10.1203/PDR.0b013e3182111827.

6. Onderdonk AB, Delaney ML, DuBois AM, Allred EN, Leviton A, Extremely Low Gestational Age Newborns Study I. Detection of bacteria in placental tissues obtained from extremelylow gestational age neonates. Am J Obstet Gynecol. 2008;198(1):110 e1-7 DOI: 10.1016/j.ajog.2007.05.044.

7. Oh KJ, Lee KA, Sohn YK, Park CW, Hong JS, Romero R, Yoon BH. Intraamniotic infection with genital mycoplasmas exhibits a more intense inflammatory response than intraamniotic infection with other microorganisms in patients with preterm premature rupture of membranes. Am J Obstet Gynecol. 2010;203(3):211 e1-8 DOI: 10.1016/j.ajog.2010.03.035.

8. Larsen B, Hwang J. Mycoplasma, Ureaplasma, and adverse pregnancy outcomes: a fresh look. Infect Dis Obstet Gynecol. 2010;2010 DOI: 10.1155/2010/521921.

9. Hegyi P, Maleth J, Walters JR, Hofmann AF, Keely SJ. Guts and Gall: Bile Acids in Regulation of Intestinal Epithelial Function in Health and Disease. Physiol Rev. 2018;98(4):1983-2023 DOI: 10.1152/physrev.00054.2017.

10. Hulzebos CV, van Zoonen AG, Hulscher JB, Schat TE, Kooi EM, Koehorst M, Boverhof R, Krabbe PF, Groen AK, Verkade HJ. Fecal Bile Salts and the Development of Necrotizing Enterocolitis in Preterm Infants. PLoS One. 2017;12(1):e0168633 DOI: 10.1371/journal.pone.0168633.

11. Halpern MD, Dvorak B. Does abnormal bile acid metabolism contribute to NEC? Semin Perinatol. 2008;32(2):114-21 DOI: 10.1053/j.semperi.2008.01.005.

12. Cherrington NJ, Estrada TE, Frisk HA, Canet MJ, Hardwick RN, Dvorak B, Lux K, Halpern MD. The hepatic bile acid transporters Ntcp and Mrp2 are downregulated in experimental necrotizing enterocolitis. Am J Physiol Gastrointest Liver Physiol. 2013;304(1):G48-56 DOI: 10.1152/ ajpgi.00317.2012.

13. Halpern MD, Weitkamp JH, Mount Patrick SK, Dobrenen HJ, Khailova L, Correa H, Dvorak B. Apical sodium-dependent bile acid transporter upregulation is associated with necrotizing enterocolitis. Am J Physiol Gastrointest Liver Physiol. 2010;299(3):G623-31 DOI: 10.1152/ajpgi.00242.2010.

14. Halpern MD, Holubec H, Saunders TA, Dvorak K, Clark JA, Doelle SM, Ballatori N, Dvorak B. Bile acids induce ileal damage during experimental necrotizing enterocolitis. Gastroenterology. 2006;130(2):359-72 DOI: 10.1053/j.gastro.2005.10.023.

15. Halpern MD, Holubec H, Dominguez JA, Meza YG, Williams CS, Ruth MC, McCuskey RS, Dvorak B. Hepatic inflammatory mediators contribute to intestinal damage in necrotizing enterocolitis. Am J Physiol Gastrointest Liver Physiol. 2003;284(4):G695-702 DOI: 10.1152/ajpgi.00353.2002. 
16. van Gorp C, de Lange IH, Spiller OB, Dewez F, Cillero Pastor B, Heeren RMA, Kessels L, Kloosterboer $\mathrm{N}$, van Gemert WG, Beeton ML, Stock SJ, Jobe AH, Payne MS, Kemp MW, Zimmermann LJ, Kramer BW, Plat J, Wolfs T. Protection of the Ovine Fetal Gut against Ureaplasma-Induced Chorioamnionitis: A Potential Role for Plant Sterols. Nutrients. 2019;11(5) DOI: 10.3390/nu11050968.

17. Bieghs V, Vlassaks E, Custers A, van Gorp PJ, Gijbels MJ, Bast A, Bekers O, Zimmermann LJ, Lutjohann D, Voncken JW, Gavilanes AW, Kramer BW, Shiri-Sverdlov R. Chorioamnionitis induced hepatic inflammation and disturbed lipid metabolism in fetal sheep. Pediatr Res. 2010;68(6):46672 DOI: 10.1203/PDR.0b013e3181f70eeb.

18. Gylling H, Plat J, Turley S, Ginsberg HN, Ellegard L, Jessup W, Jones PJ, Lutjohann D, Maerz W, Masana L, Silbernagel G, Staels B, Boren J, Catapano AL, De Backer G, Deanfield J, Descamps OS, Kovanen PT, Riccardi G, Tokgozoglu L, Chapman MJ, European Atherosclerosis Society Consensus Panel on P. Plant sterols and plant stanols in the management of dyslipidaemia and prevention of cardiovascular disease. Atherosclerosis. 2014;232(2):346-60 DOI: 10.1016/j. atherosclerosis.2013.11.043.

19. Bouic PJ, Lamprecht JH. Plant sterols and sterolins: a review of their immune-modulating properties. Altern Med Rev. 1999;4(3):170-7.

20. Ding Y, Nguyen HT, Kim Sl, Kim HW, Kim YH. The regulation of inflammatory cytokine secretion in macrophage cell line by the chemical constituents of Rhus sylvestris. Bioorg Med Chem Lett. 2009;19(13):3607-10 DOI: 10.1016/j.bmcl.2009.04.129.

21. Valerio M, Awad AB. beta-Sitosterol down-regulates some pro-inflammatory signal transduction pathways by increasing the activity of tyrosine phosphatase SHP-1 in J774A.1 murine macrophages. Int Immunopharmacol. 2011;11(8):1012-7 DOI: 10.1016/j.intimp.2011.02.018.

22. Valerio MS, Minderman H, Mace T, Awad AB. beta-Sitosterol modulates TLR4 receptor expression and intracellular MyD88-dependent pathway activation in J774A.1 murine macrophages. Cell Immunol. 2013;285(1-2):76-83 DOI: 10.1016/j.cellimm.2013.08.007.

23. Kim KA, Lee IA, Gu W, Hyam SR, Kim DH. beta-Sitosterol attenuates high-fat diet-induced intestinal inflammation in mice by inhibiting the binding of lipopolysaccharide to toll-like receptor 4 in the NFkappaB pathway. Mol Nutr Food Res. 2014;58(5):963-72 DOI: 10.1002/mnfr.201300433.

24. Aldini R, Micucci M, Cevenini M, Fato R, Bergamini C, Nanni C, Cont M, Camborata C, Spinozzi S, Montagnani M, Roda G, D'Errico-Grigioni A, Rosini F, Roda A, Mazzella G, Chiarini A, Budriesi R. Antiinflammatory effect of phytosterols in experimental murine colitis model: prevention, induction, remission study. PLoS One. 2014;9(9):e108112 DOI: 10.1371/journal.pone.0108112.

25. Mencarelli A, Renga B, Palladino G, Distrutti E, Fiorucci S. The plant sterol guggulsterone attenuates inflammation and immune dysfunction in murine models of inflammatory bowel disease. Biochem Pharmacol. 2009;78(9):1214-23 DOI: 10.1016/j.bcp.2009.06.026.

26. te Velde AA, Brull F, Heinsbroek SE, Meijer SL, Lutjohann D, Vreugdenhil A, Plat J. Effects of Dietary Plant Sterols and Stanol Esters with Low- and High-Fat Diets in Chronic and Acute Models for Experimental Colitis. Nutrients. 2015;7(10):8518-31 DOI: 10.3390/nu7105412.

27. Cheon JH, Kim JS, Kim JM, Kim N, Jung HC, Song IS. Plant sterol guggulsterone inhibits nuclear factor-kappaB signaling in intestinal epithelial cells by blocking IkappaB kinase and ameliorates acute murine colitis. Inflamm Bowel Dis. 2006;12(12):1152-61 DOI: 10.1097/01. mib.0000235830.94057.c6.

28. Plat J, Hendrikx T, Bieghs V, Jeurissen ML, Walenbergh SM, van Gorp PJ, De Smet E, Konings M, Vreugdenhil AC, Guichot YD, Rensen SS, Buurman WA, Greve JW, Lutjohann D, Mensink RP, ShiriSverdlov R. Protective role of plant sterol and stanol esters in liver inflammation: insights from mice and humans. PLoS One. 2014;9(10):e110758 DOI: 10.1371/journal.pone.0110758. 
29. Pfisterer C, Faber R, Horn LC. Chorioamnionitis-induced changes of fetal extramedullar hematopoiesis in the second trimester of gestation. Is diagnosis from fetal autopsy possible? Virchows Arch. 2005;446(2):150-6 DOI: 10.1007/s00428-004-1151-9.

30. Brancatelli G, Furlan A, Calandra A, Dioguardi Burgio M. Hepatic sinusoidal dilatation. Abdom Radiol (NY). 2018;43(8):2011-22 DOI: 10.1007/s00261-018-1465-8.

31. Chiang JY. Bile acid metabolism and signaling. Compr Physiol. 2013;3(3):1191-212 DOI: 10.1002/ cphy.c120023.

32. Zimmer S, Grebe A, Bakke SS, Bode N, Halvorsen B, Ulas T, Skjelland M, De Nardo D, Labzin LI, Kerksiek A, Hempel C, Heneka MT, Hawxhurst V, Fitzgerald ML, Trebicka J, Bjorkhem I, Gustafsson JA, Westerterp M, Tall AR, Wright SD, Espevik T, Schultze JL, Nickenig G, Lutjohann D, Latz E. Cyclodextrin promotes atherosclerosis regression via macrophage reprogramming. Sci Transl Med. 2016;8(333):333ra50 DOI: 10.1126/scitransImed.aad6100.

33. Bakke SS, Aune MH, Niyonzima N, Pilely K, Ryan L, Skjelland M, Garred P, Aukrust P, Halvorsen B, Latz E, Damas JK, Mollnes TE, Espevik T. Cyclodextrin Reduces Cholesterol Crystal-Induced Inflammation by Modulating Complement Activation. J Immunol. 2017;199(8):2910-20 DOI: 10.4049/jimmunol.1700302. 


\section{CHAPTER 7}

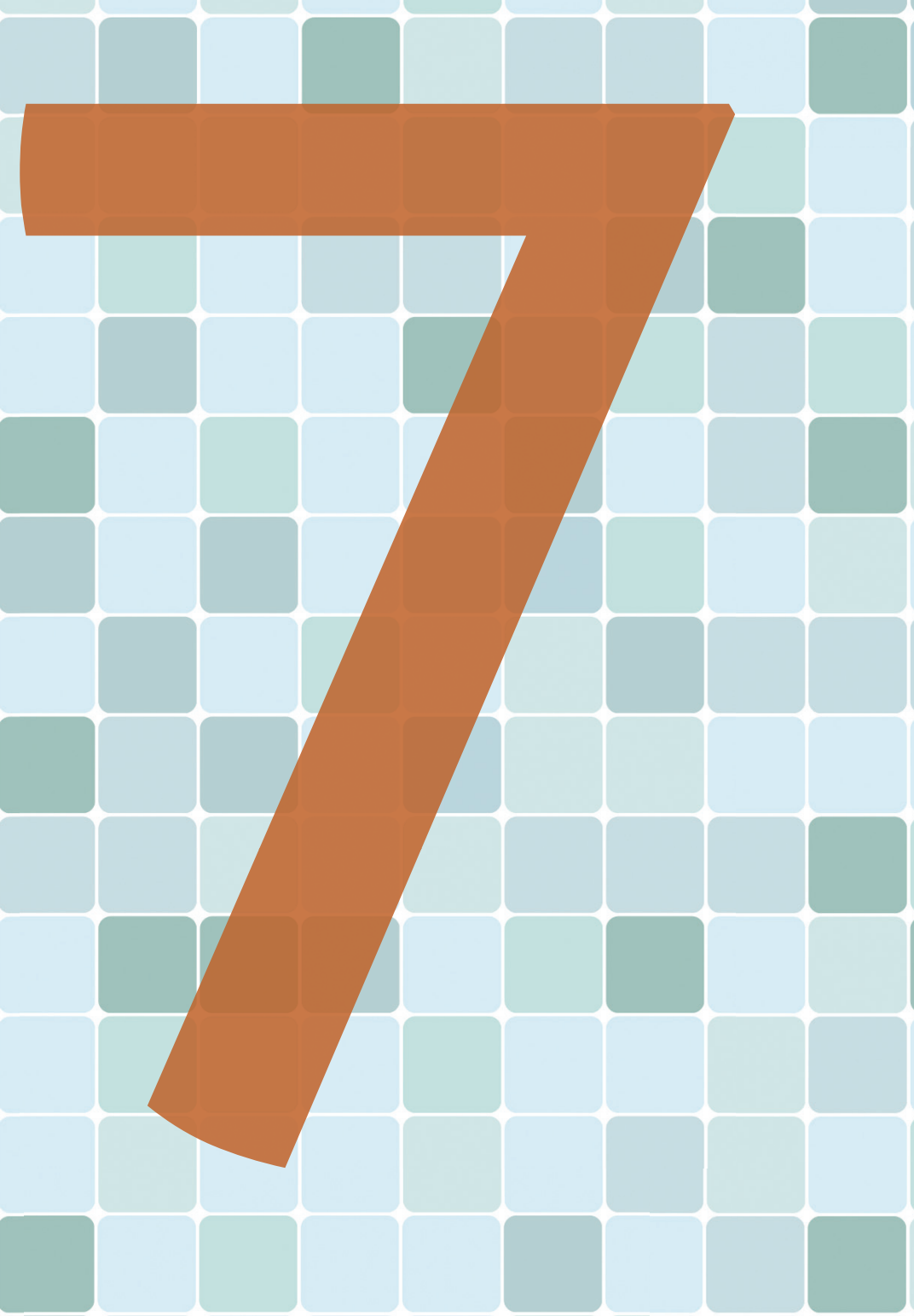


General discussion, implications and future perspectives 



\section{General discussion}

Chorioamnionitis is, regardless of preterm birth, an independent risk factor for adverse neonatal outcomes $(1,2)$, including necrotizing enterocolitis (NEC) (2-4). At present, the multifactorial pathogenesis of NEC is still incompletely understood.

One of the suspected pathophysiological mechanisms contributing to NEC development is an immature gut motility, facilitating abnormal bacterial colonization $(5,6)$. Various clinical signs of gut dysmotility such as vomiting, feeding intolerance and abdominal distention have been reported in a rodent NEC model (7). Moreover, functional and structural enteric nervous system (ENS) abnormalities of both the submucosal and myenteric plexus have been found in intestinal biopsies from NEC patients and pre-clinical models (8-12). More precisely, loss of enteric neurons and glial cells was observed in both the submucosal and myenteric plexus (8-12). These ENS alterations have been investigated in more detail in a porcine NEC model, a study in which region-dependent changes in gut transit time were observed before radiological signs of NEC appeared, suggesting that dysmotility precedes NEC development (13). Taken together, these data support the general concept that a disturbed intestinal motility is causally related to NEC pathophysiology.

Another pathophysiological mechanism that may contribute to NEC development is ileal damage caused by elevated ileal bile acids (BAs) in the lumen and enterocytes as a result of an altered expression of several BAs transporters (14-18). A role for BAs in the pathogenesis of NEC is further supported by the finding that fecal BA levels were higher in preterm infants in the week preceding NEC manifestation compared to gestationmatched controls (18). Since in utero circumstances (e.g. chorioamnionitis) influence NEC risk, alterations in the enterohepatic circulation (EHC) might originate in utero. The important role of the liver and the gut-liver axis in NEC pathogenesis is further underlined by the presence of increased hepatic inflammation in neonatal rats with NEC, which correlated with the progression of intestinal damage during disease development (19).

This thesis focused on examining the effect of an intra-uterine infection on the ENS, liver and EHC of the fetus to gain essential insight into the relationship between chorioamnionitis and the predisposition to adverse gut and liver outcomes including NEC. First, we investigated the effect of an Ureaplasma parvum- as well as an endotoxininduced intra-amniotic (IA) infection on ENS development, studying both the effect of time of onset and the duration of an IA infection. Second, we evaluated postnatal ENS and intestinal motility changes in a NEC mouse model to investigate the effects of perinatal stress after birth. Third, the effect of an IA infection on the liver and EHC was investigated. In addition, we investigated the potential of prophylactic IA plant sterol administration to prevent chorioamnionitis-induced liver inflammation and potential EHC alterations. Plant sterols have been shown to have immune-modulatory effects in the healthy and inflamed intestine and liver and may thereby modulate the gastrointestinal and liver outcome following chorioamnionitis (20-30). 


\section{The effect of chorioamnionitis on the enteric nervous system}

As described above, ENS abnormalities are found in neonates with NEC and in rodent NEC models (8-12). In chapter $\mathbf{2}$ and 3, we questioned whether ENS alterations originate already, at least in part, in utero as a result of chorioamnionitis.

First, we explored the effects of acute IA lipopolysaccharide (LPS) and chronic IA Ureaplasma parvum (UP) exposure on the ENS of the terminal ileum in premature lambs in chapter 2. We showed that the timing of the inflammatory challenge during the vulnerable second trimester, which is essential for both morphological and functional maturation of the ENS $(31,32)$, appeared to be the key determinant for the loss of both mature enteric neurons and glial cells $(31,32)$.

Secondly, in chapter 2, we investigated the effects of repetitive exposure to UP and LPS on the ENS. The potential interplay between different microbial stimuli is clinically important, as chorioamnionitis is often poly-microbial (33). In addition, inflammation as driving force behind perinatal organ injury, may be induced, sustained, and aggravated by multiple perinatal factors interacting in a multiple-hit sequence. Interestingly, consecutive exposure to two different microbial stimuli did not affect the number of enteric glial cells and enteric neurons compared to control, indicating that glial cells can withstand this stressor, and because of this also can protect the neurons in these circumstances. These results suggest that enteric glial cells are important for neuronal survival and neurogenesis in the intra-uterine setting.

In chapter 3, IA infection with LPS was initiated at various time points to investigate the time-dependent ENS changes following intra-uterine inflammation. Chorioamnionitis is often clinically silent and infants born after chorioamnionitis may have been exposed to varying durations of intra-uterine microbial stimuli at the time of diagnosis. Interestingly, both UP (chapter 2) and LPS (chapter $\mathbf{3}$ ) induced chorioamnionitis were associated with a loss of enteric neurons and glial cells, suggesting that different microbial triggers can induce similar ENS damage. The work described in chapter 3 suggests that fetal systemic inflammation following IA infection is an important determinant for the loss of enteric neurons and glial cells. In addition, these ENS alterations might also result from direct exposure of the intestine to bacterial components and inflammatory cytokines present in the amniotic fluid, which is swallowed by the fetus.

Importantly, our findings are supported by previous studies in rodent NEC models and neonates with NEC demonstrating loss of enteric neurons and glial cells in the submucosal and myenteric plexus (8-12). It has been suggested that ablation of enteric glial cells may lead to NEC pathology (34). Enteric glial cells are important for neuronal maintenance, survival, and function (35) and are capable of generating enteric neurons in response to injury $(36,37)$. Combined, these data suggest that glial cells are important for neuronal survival and neurogenesis, also in the intra-uterine setting, and that loss of these cells in utero potentially predisposes to NEC.

Since the most profound ENS changes in chapter $\mathbf{2}$ and $\mathbf{3}$ were found in the myenteric plexus, which is primarily responsible for coordinating contractions and relaxations 
of gut smooth muscle involved in gut motility (38), one can expect adverse intestinal motility outcomes. It is tempting to speculate that functions of the submucosal plexus, responsible for e.g. coordinating the exchange of fluids across the mucosal surface, secretion of hormones, absorption and local blood flow, were retained since no loss of enteric neurons and glial cells was found in this part of the ENS. However, the exact postpartum functions of the ENS after antenatal infection remain to be elucidated. It would be interesting to investigate longitudinal ENS outcomes post-partum in lambs that were IA exposed to a microbial stimulus as additional postnatal inflammatory hits that a premature born child is likely to encounter, such as early onset infection or sepsis, oxygen toxicity, mechanical ventilation (39-44), which may further harm the ENS and negatively influence its development, recovery and functional outcomes. The post-partum outcomes should be investigated in conjunction with the microbiome, as recent studies indicate that gut microbiota diversification, which occurs alongside postnatal ENS maturation, could be critical for the development and function of the ENS (45).

As an essential intermediate step to bridge the gap between post-partum outcomes and additional postnatal inflammatory hits (as the mouse intestine and ENS is still immature at birth $(46,47)$ ), we evaluated the consequences of perinatal inflammatory stress on the ENS after birth in a murine NEC model in chapter 4. In this model, NEC was induced in newborn mice by formula feeding, oral commensal bacteria with LPS supplementation, asphyxia and cold stress. Importantly, in this rodent model, we correlated histological findings with functional consequences (intestinal transit).

In contrast to the findings described in chapter $\mathbf{2}$ and $\mathbf{3}$ and the ENS changes found in previous studies in rodent NEC models and neonates with NEC, the study in chapter 4 revealed an increased number of mature neurons and enteric glial cells in mice exposed to the NEC protocol. These histological findings are in accordance with observations of hypertrophy and hyperplasia of neuronal cell bodies and hyperplasia of glial cells in inflammatory bowel disease, characterized by intestinal inflammation, but typically no necrosis (48). It is therefore tempting to speculate that our data may represent a relatively milder disease state with an ENS response that predisposes to glial cell and neuronal loss at a later time point.

In addition, in chapter 4, we observed an increased synaptophysin-positive surface area in the submucosal and myenteric plexus of animals subjected to the NEC protocol. In line, we initially observed an increased intestinal transit, indicating increased intestinal motility. This most likely results from an immunological host response and subsequent diarrhoea (49-52). Alternatively, this might reflect a functional consequence of an increased presence of presynaptic vesicles (synaptophysin immunoreactivity). In contrast, 48 hours after exposure to the NEC protocol, the intestinal transit decreased, implying decreased intestinal motility in these animals. Possibly, neurons were initially able to compensate and react to intestinal inflammation, but in a later stage, despite the neurons still being able to produce synaptophysin, motility decreased. These findings indicate that additional studies are needed to unravel the mechanisms behind the altered intestinal motility following exposure to the NEC protocol. 
In summary, although our in utero ENS alterations provide strong evidence of a dysregulated enteric network and thus disrupted ENS functions following chorioamnionitis, future studies are warranted to assess intestinal motility postnatally after IA infections initiated at various time points. For this purpose, postnatal in vivo evaluation of intestinal motor function after IA infection and potential other pro-inflammatory hits could link the current in utero findings of loss of enteric neurons and glial cells with the observed disrupted ENS functions in the rodent NEC model.

\section{The effect of chorioamnionitis on the liver and enterohepatic circulation of bile acids}

The liver is increasingly recognized as primary immune organ during early stages of development $(53,54)$. Hepatic macrophages are able to capture pathogens and antigens in the bloodstream due to their unique location outside of the sinusoidal space. This way, they are uniquely positioned to initiate an innate immune response to a systemic infection (55-57). Following activation, the hepatic macrophages send signals to other organs through the production and systemic release of cytokines (58). In chapter 5, we indeed detected an early hepatic response after IA LPS injections; hepatic cytokine production occurred from 5 hours until 2 days after IA LPS administration. These time points overlapped with the ongoing fetal systemic inflammatory response (FIRS; characterized by increased circulatory IL-6 levels $(30,59)$ ), suggesting that FIRS and local cytokine production in the liver are causally linked. Indeed, in a recent study in a similar sheep chorioamnionitis model, hepatic cytokine production was found already 1 hour, and similar to our findings also at 5 hours, after IA LPS injection (60). According to these results, the liver might thus be one of the first organs to induce an innate immune response by early cytokine production as a result of chorioamnionitis. The exact mechanism of how IA LPS can produce an early hepatic innate immune response activation remains to be determined. Interestingly, in a small sub-study, the presence of circulating LPS metabolites was already detected 15 min after IA LPS exposure (60), which could be an explanation for the early hepatic immune response.

Increased production of hepatic cytokines, which are released into the biliary system, was also found in neonatal rats with NEC and correlated with the progression of intestinal damage during disease development (19). In chapter 2 and 5, mucosal and submucosal inflammation was observed in the terminal ileum at day 2 and 4 after IA LPS administration. Although hepatic TNF-a, IL-8 and IL-18 levels were normalized at day 4 after IA LPS administration, it is possible that the early hepatic cytokine response contributed to the intestinal inflammation and development of NEC.

Histologic hepatic inflammation was observed as a result of an antenatal infection, i.e. LPS (chapter 5) and UP (chapter 6). It was characterized by an increased number of extramedullary hematopoietic clusters, in which specific, clustered and conflated hepatic erythropoiesis was observed in the liver parenchyma. Interestingly, in human stillborns, a similar histological pattern was strongly associated with chorioamnionitis (61), implicating that antenatal inflammation affects fetal extramedullary hematopoiesis. Extramedullary hematopoiesis in the liver is known to occur as a normal response to inflammation and infection, which results in the production of phagocytic cells and antigen-presenting 
cells (62). The early hepatic cytokine production, ongoing FIRS or the direct exposure to inflammatory mediators through transport from the gut via the portal vein, or a combination of these are likely the cause of the observed altered fetal extramedullary hematopoiesis.

The liver and intestine are closely connected via the EHC. In line, BAs are recently found to be critical regulators of intestinal epithelial function (63). Several studies have found that elevated ileal BAs and an altered expression of several BAs transporters may result in ileal damage, contributing to the pathophysiology of (experimental) NEC (14-18). Given the role of BAs in NEC pathogenesis and the fact that fecal unconjugated BA levels are higher in preterm infants in the week preceding NEC manifestation compared with gestation matched control (18), we hypothesized that alterations in the $\mathrm{EHC}$ have their origin in utero. In this context, we further evaluated the time-dependent effects of IA administration of LPS in chapter $\mathbf{5}$ and IA UP exposure in chapter $\mathbf{6}$ on the EHC of premature sheep.

In chapter 5, total ileal BA concentrations remained unchanged at different time points after IA LPS administration. In line, in a more recent study in which NEC infants were compared to matched controls, no differences in total fecal BAs nor differences in BA species were found (64). However, in the month prior to NEC diagnosis, a difference in the mean coefficient of variation of total BAs was detected when compared to controls (64). This latter finding indicates that fluctuations in fecal BA levels were measured, which suggests that the neonatal ileum may be especially sensitive to pulsatile exposure to BAs in the first month, potentially contributing to NEC development (64).

Interestingly, in the same model as used in chapter 6, a reduction of the conjugated BAs taurocholic acid (TCA), taurochenodeoxycholic acid (TCDCA) and taurodeoxycholic acid (TDCA) in the enterocytes was found after six days of IA UP exposure by using mass spectrometry imaging (30). This might be a sign that fluctuations of BAs over time as in the study of Knapp et al. can occur in utero. Currently, the arguments that can explain this difference remain unclear. It is tempting to speculate that the microbial trigger appears to be an essential determinant in BA homeostasis following an IA infection.

In the normal situation, BAs repress ASBT to prevent toxicity from intracellular accumulation of BAs $(14,16)$. However, it is thought that increased cytokine levels can increase the expression of ASBT, which leads to accumulation of intra-enterocyte BAs, resulting in increased incidence and severity of (experimental) NEC (14-16, 18, 65, 66). In chapter 5, we found at two different time points (24h and 8d after IA LPS exposure) an increased expression of ASBT, which might be related to the observed increased ileal cytokine levels such as TNF- $a$ and IL-18 levels, which in previous studies have been shown to upregulate ASBT expression $(65,66)$. Despite increased ASBT expression, ileal $B A s$ remained unchanged. Moreover, in chapter 6 , ASBT expression remained unchanged after IA UP exposure, while a reduction of conjugated BAs in the ileum was found in the same model (30), indicating that further investigation into ASBT regulation and fluctuating ileal BA levels is necessary. Future studies should measure ileal BA levels, ASBT levels and post-partum fecal BAs (within one animal) at multiple time points after IA UP and/or 
LPS administration to further unravel the role of BAs in the context of adverse neonatal outcomes following perinatal infections.

Altogether, in chapter $\mathbf{5}$ and $\mathbf{6}$, we did not find elevated ileal BAs in combination with the increased ASBT expression as was previously found in experimental NEC and neonates with NEC $(14-16,18)$. However, we did find an increased expression of ASBT at different time points, suggesting that the EHC alterations contributing to NEC pathology might in part have their origin in utero.

\section{Treatments}

In this thesis, we showed that chorioamnionitis-induced FIRS is associated with hepatic inflammation and alterations of the ENS and EHC. This finding could mechanistically explain why chorioamnionitis-induced FIRS (59) is a risk factor for neonatal morbidity, including NEC $(4,67)$. Since our data and previous literature suggest that early hepatic cytokine production can induce FIRS, the fetal hepatic innate immune response must be considered when developing therapeutic approaches. In this context, immune modulatory interventions that can target systemic inflammation and/or inhibit inflammation at organ level hold great promise. The clinical potential of this concept was shown by several interventions, i.e. IA recombinant human IL-1 receptor antagonist (rhIL1 ra), prophylactic IL-2 and prophylactic plant sterol supplementation. These interventions have been shown to inhibit chorioamnionitis-induced FIRS and/or inhibit inflammation and damage at organ level, i.e. fetal lung and intestine $(30,68-70)$. Future studies in the same models should investigate whether above immune modulatory interventions can also prevent hepatic inflammation and alterations of the ENS and EHC to attenuate organ injury associated with in utero inflammation.

A noninvasive intervention without the necessity to enter the IA cavity (due to the risk of abortion) would be most desired. A safe and accessible immune modulatory intervention would therefore be enrichment of the mother diet by plant sterols, as they are naturally present in breast feeding (71-73) and amniotic fluid $(74,75)$. From a treatment perspective, plant sterols seem promising due to their immune-modulatory properties $(20-27,29,76-$ 79). Several in vitro studies have supported immune modulation by plant sterols on both the innate and the acquired immune system, in which the secretion of pro-inflammatory mediators by macrophages was suppressed by plant sterols (20, 22, 24, 25, 27, 76-79).

In the fetal gut, anti-inflammatory effects of plant sterols were previously demonstrated in an ovine chorioamnionitis model (30). Plant sterols also decreased UP-induced systemic inflammation (30). Moreover, plant sterols and/or the carrier $\beta$-cyclodextrin reduced mucosal damage and stimulated the maturation of enterocytes (30). In the liver of mice that developed non-alcoholic fatty liver disease (NASH) (28), plant sterols have also shown to be anti-inflammatory. However, some safety concerns were raised by the occurrence of intestinal failure-associated liver disease (IFALD), characterized by hepatic inflammation (increased hepatic cytokine production) and decreased expression of BSEP promoting cholestasis and hepatic cholestatic injury in neonates that received long-term parenteral nutrition containing high concentrations of plant sterols (80-84). 
Above studies in NASH and IFALD describe different routes of plant sterol administration, of which enteral administration seems the best route since in the mouse NASH study anti-inflammatory effects of enteral administrated plant sterols were demonstrated (28). As the fetus swallows the amniotic fluid, IA administration will eventually result in enteral uptake of plant sterols. Hence, in chapter 6, we aimed to investigate the effects of prophylactic IA plant sterols (a mixture of $\beta$-sitosterol and campesterol dissolved in the carrier $\beta$-cyclodextrin) on the liver and EHC in the IA UP chorioamnionitis model. In this study, there were no signs of IA plant sterols causing hepatic inflammation, decreased expression of BSEP or cholestasis. In addition, fetal plasma plant sterol concentrations were not increased by the IA treatment (30). This could be related to the IA (therefore enteral) administration of the plant sterols. Also other factors should be considered, such as dosage and duration of plant sterol administration in our study.

Interestingly, in this study, both plant sterols dissolved in the carrier and the carrier alone possessed similar anti-inflammatory effects, suggesting that the anti-inflammatory effects in the liver might be solely the result of the carrier $\beta$-cyclodextrin. This is in contrast with the anti-inflammatory effects of plant sterols in the fetal gut, which were previously demonstrated in the same ovine chorioamnionitis model (30). We cannot fully explain these different therapeutic effects of the carrier $\beta$-cyclodextrin in the liver in chapter 6 and the intestinal effects of plant sterols and the carrier $\beta$-cyclodextrin in the same model. Interestingly, $\beta$-cyclodextrin was previously shown to exert anti-inflammatory effects in atherosclerosis (85-87), mediated by among others an increase in liver-X-receptor (LXR) target gene expression in macrophages, which in turn lowered the systemic levels of LXR-modulated cytokines (IL-1 $\beta$, IL-6 and TNF- $\alpha$ ) (87). Plant sterols are also known activators of LXRs (88), and decreased LXR expression is observed in experimental colitis and inflammatory bowel disease (89). Moreover, $\beta$-cyclodextrin is capable of trapping cholesterol and lipids and therewith removing intracellular cholesterol and lipids that can cause injury and inflammation to various organs (90). In chapter 6, IA $\beta$-cyclodextrin administration partly decreased hepatic inflammation during UPinduced chorioamnionitis. However, this was not accompanied by changes in the hepatic cholesterol pool as fetal circulatory lathosterol concentrations, that are indicative for (hepatic) endogenous cholesterol synthesis, remained unaltered (30). Finally, the liver cholesterol pool was sufficient to such extent that it did not have a negative impact on the hepatic synthesis of primary BAs, suggesting that antenatal IA $\beta$-cyclodextrin administration does not harm the cholesterol pool and BAs synthesis in utero. Considering all the above, future studies are required to investigate the exact working mechanisms in the fetus to further dissect the role of $\beta$-cyclodextrin in the protection of the fetal liver during chorioamnionitis and ultimately unravel the clinical potential of $\beta$-cyclodextrin in the perinatal context.

In conclusion, the studies described in this thesis demonstrate that chorioamnionitis can induce intestinal inflammation, which coincided with ENS alterations, especially when infection is induced early in pregnancy. Postnatally, in a NEC mouse model, ENS and intestinal motility alterations were also observed. Although, we did not explore whether the in utero observed ENS alterations result in functional abnormalities after birth, the observed changes correspond with clinical and histological findings in neonates with 
NEC, which underlines the concept that NEC pathophysiology might start in utero. In addition, one of our studies using the chorioamnionitis model demonstrated an increased expression of ASBT at different time points, suggesting that the EHC alterations contributing to NEC pathology might in part have their origin in utero. Lastly, we provided evidence that chorioamnionitis can induce early hepatic cytokine production, which was earlier shown to contribute to a systemic innate immune response. Moreover, hepatic inflammation was observed as a result of chorioamnionitis, which could be partially prevented by prophylactic IA $\beta$-cyclodextrin administration.

\section{Implications and future perspectives}

This thesis focused on the effect of an antenatal intra-uterine infection on the ENS, liver and $\mathrm{EHC}$ of the fetus in order to gain essential insight in the relation between chorioamnionitis and the predisposition to adverse gut and liver outcomes including NEC.

We demonstrated that chorioamnionitis results in ENS changes in utero and that the vulnerability of the fetus to injurious IA triggers is determined by the onset and duration of these triggers. These ENS changes may increase susceptibility to intestinal injury later in life and may explain why preterm infants exposed to chorioamnionitis prior to birth have a higher incidence of NEC. Although the ENS alterations in utero provide strong evidence of a dysregulated enteric network and thus disrupted ENS functions following chorioamnionitis, future studies are warranted to assess intestinal motility postnatally. Indeed, postnatal in vivo evaluation of intestinal motor function after chorioamnionitis could link the current in utero findings with the clinical observations of decreased motility, facilitating intraluminal bacterial overgrowth and translocation, in neonates suffering from NEC.

As shown in chapter $\mathbf{2} \mathbf{- 4}$ initiation of an intestinal inflammatory response leads to ENS injury. Modulating this inflammatory response directly or indirectly is considered to be a key treatment target. Second, regeneration of injured enteric neurons and glial cells and/ or stimulation of the maturation of enteric neurons is a prerequisite to repair ENS injury. In addition, the data presented in chapter $\mathbf{2}$ indicate that there is a defined window of vulnerability in which additional inflammation may have a greater impact as the ENS is already affected at that time, and in which therapeutic interventions could be very well initiated. The effect of timing of treatment initiation on the ENS should be further studied.

In literature, several promising treatments have been proposed as treatments for the ENS alterations found in NEC, such as the administration of heparin-binding epidermal growth factor-like growth factor, neuronal stem cell transplantation and ghrelin (11, $12,91)$. These treatments were able to recover the NEC-induced loss of enteric neurons and glial cells, promote enteric nerve regeneration, by for instance promoting glial cellderived neurogenesis, and improve intestinal transit $(11,12,91)$. Future studies should focus on applying these treatments in the intra-uterine setting during chorioamnionitis to evaluate whether ENS alterations can be repaired or prevented, also in the context of additional injurious hits (postnatally). Moreover, early detection of IA infections will 
facilitate appropriate timing of these treatments or preventative measures, which is discussed below.

We also demonstrated that hepatic inflammation is induced in utero as a result of chorioamnionitis and that prophylactic IA $\beta$-cyclodextrin administration can partly prevent the hepatic inflammatory response. A previous study from our group found that plant sterols reduce chorioamnionitis-induced gut inflammation and that $\beta$-cyclodextrin reduces chorioamnionitis-induced gut injury (30). Based on these earlier findings and the results of our study, we conclude that $\beta$-cyclodextrin is an interesting pharmaceutical target to protect the gut and liver against the negative consequences of perinatal inflammatory stress $(87,92)$. Importantly, cyclodextrins are highly water soluble, "readymade", and commercially available. Its pharmacokinetics, the optimal moment and route of administration, side effects and safety in the fetal or neonatal context warrant further investigation. Future studies should focus on the correct timing and dose of IA $\beta$-cyclodextrin administration in utero during chorioamnionitis.

Altogether, in chapter 5 and 6, we did not find elevated ileal BAs in combination with increased ASBT expression as was previously found in experimental NEC and neonates with NEC $(14-16,18)$. However, we did find an increased expression of ASBT at different time points, suggesting that the EHC alterations contributing to NEC pathology might partly have their origin in utero. Besides, based on these studies, we cannot exclude the fact that the observed hepatic and intestinal inflammation in utero as a result of chorioamnionitis might predispose to more permanent EHC alterations after birth. In a previous ovine chorioamnionitis study, it was shown that antenatal exposure to chorioamnionitis affected lipid metabolism in 7-week-old sheep (93), demonstrating that chorioamnionitis can alter liver function post-partum. Future studies should focus on the postnatal consequences of chorioamnionitis-induced hepatic and intestinal inflammation and FIRS on the EHC to get more insight in the relationship between chorioamnionitis and the predisposition to NEC; e.g. measuring fecal BAs at various time points within one animal.

Chapter 6 and several pre-clinical studies showed that interventions at the earliest possible moment, being in utero, have been successfully tested (94-102). Successful development and implementation of such treatment strategies in clinical care is currently impeded by a lack of diagnostic tools for early (prenatal) detection and surveillance of intra-amniotic infections. Currently, the gold standard to diagnose chorioamnionitis is histological examination after birth with proof of inflammation and necrosis throughout the chorionic plate and amnion (103, 104). Ideally, a noninvasive maternal marker to detect chorioamnionitis, without performing an amniocentesis (due to the risk of abortion), would allow timely interventions to prevent intestinal inflammation, ENS damage, liver inflammation and EHC alterations. There have been some attempts to detect biomarkers specific for chorioamnionitis in the maternal circulation, e.g. IL-6, IL8, C-reactive protein (CRP), ferritin and microbial cell-free DNA, however, they have not yet been proven reliable to be translated into the clinic $(105,106)$. Moreover, a recent study analyzed volatile organic compounds (VOCs), which are formed during various inflammatory and metabolic processes on cellular and systemic level (107), in exhaled breath of pregnant sheep (108). This study showed that an UP infection can be detectable 
with good accuracy using exhaled breath profile 96 hours post infection (108). These data show great potential to identify pregnancies complicated by IA infections by VOCs analysis and clinical implementation would be an immense breakthrough in perinatal diagnostics. Early detection of IA infections will lead to optimal surveillance of these highrisk pregnancies and will facilitate appropriate clinical management including timely treatment or preventative measures for prematurity-associated morbidities.

In conclusion, in our translational chorioamnionitis ovine models, we demonstrated that prenatal events induce intestinal inflammation, ENS damage, liver inflammation and EHC alterations, potentially predisposing to NEC. We have provided evidence on a potential intervention that is able to decrease the inflammatory response in the liver. The results presented in this thesis form the basis for future studies in which therapeutic strategies for gut- and liver-associated complications of chorioamnionitis can be tested. 


\section{References}

1. Viscardi RM. Ureaplasma species: role in diseases of prematurity. Clin Perinatol. 2010;37(2):393409 DOI: 10.1016/j.clp.2009.12.003.

2. SilwedelC,SpeerCP, Glaser K.Ureaplasma-associated prenatal, perinatal, and neonatalmorbidities. Expert Rev Clin Immunol. 2017;13(11):1073-87 DOI: 10.1080/1744666x.2017.1381559.

3. Okogbule-Wonodi AC, Gross GW, Sun CC, Agthe AG, Xiao L, Waites KB, Viscardi RM. Necrotizing enterocolitis is associated with ureaplasma colonization in preterm infants. Pediatr Res. 2011;69(5 Pt 1):442-7 DOI: 10.1203/PDR.0b013e3182111827.

4. Been JV, Lievense S, Zimmermann LJ, Kramer BW, Wolfs TG. Chorioamnionitis as a risk factor for necrotizing enterocolitis: a systematic review and meta-analysis. J Pediatr. 2013;162(2):236-42 e2 DOI: 10.1016/j.jpeds.2012.07.012.

5. Lin PW, Stoll BJ. Necrotising enterocolitis. Lancet. 2006;368(9543):1271-83 DOI: 10.1016/S01406736(06)69525-1.

6. Neu J. Necrotizing enterocolitis. World Rev Nutr Diet. 2014;110:253-63 DOI: 10.1159/000358474.

7. Koike Y, Li B, Lee C, Cheng S, Miyake H, Welsh C, Hock A, Belik J, Zani A, Pierro A. Gastric emptying is reduced in experimental NEC and correlates with the severity of intestinal damage. J Pediatr Surg. 2017;52(5):744-8 DOI: 10.1016/j.jpedsurg.2017.01.031.

8. Sigge W, Wedel T, Kuhnel W, Krammer HJ. Morphologic alterations of the enteric nervous system and deficiency of non-adrenergic non-cholinergic inhibitory innervation in neonatal necrotizing enterocolitis. Eur J Pediatr Surg. 1998;8(2):87-94 DOI: 10.1055/s-2008-1071128.

9. Wedel T, Krammer HJ, Kuhnel W, Sigge W. Alterations of the enteric nervous system in neonatal necrotizing enterocolitis revealed by whole-mount immunohistochemistry. Pediatr Pathol Lab Med. 1998;18(1):57-70.

10. Fagbemi AO, Torrente F, Puleston J, Lakhoo K, James S, Murch SH. Enteric neural disruption in necrotizing enterocolitis occurs in association with myenteric glial cell CCL20 expression. J Pediatr Gastroenterol Nutr. 2013;57(6):788-93 DOI: 10.1097/MPG.0b013e3182a86fd4.

11. Zhou Y, Yang J, Watkins DJ, Boomer LA, Matthews MA, Su Y, Besner GE. Enteric nervous system abnormalities are present in human necrotizing enterocolitis: potential neurotransplantation therapy. Stem Cell Res Ther. 2013;4(6):157 DOI: 10.1186/scrt387.

12. Wei J, Zhou Y, Besner GE. Heparin-binding EGF-like growth factor and enteric neural stem cell transplantation in the prevention of experimental necrotizing enterocolitis in mice. Pediatr Res. 2015;78(1):29-37 DOI: 10.1038/pr.2015.63.

13. Chen W, Sun J, Kappel SS, Gormsen M, Sangild PT, Aunsholt L. Gut transit time, using radiological contrast imaging, to predict early signs of necrotizing enterocolitis. Pediatr Res. 2020 DOI: 10.1038/ s41390-020-0871-0.

14. Halpern MD, Holubec H, Saunders TA, Dvorak K, Clark JA, Doelle SM, Ballatori N, Dvorak B. Bile acids induce ileal damage during experimental necrotizing enterocolitis. Gastroenterology. 2006;130(2):359-72 DOI: 10.1053/j.gastro.2005.10.023.

15. Halpern MD, Dvorak B. Does abnormal bile acid metabolism contribute to NEC? Semin Perinatol. 2008;32(2):114-21 DOI: 10.1053/j.semperi.2008.01.005.

16. Halpern MD, Weitkamp JH, Mount Patrick SK, Dobrenen HJ, Khailova L, Correa H, Dvorak B. Apical sodium-dependent bile acid transporter upregulation is associated with necrotizing enterocolitis. Am J Physiol Gastrointest Liver Physiol. 2010;299(3):G623-31 DOI: 10.1152/ajpgi.00242.2010. 
17. Cherrington NJ, Estrada TE, Frisk HA, Canet MJ, Hardwick RN, Dvorak B, Lux K, Halpern MD. The hepatic bile acid transporters Ntcp and Mrp2 are downregulated in experimental necrotizing enterocolitis. Am J Physiol Gastrointest Liver Physiol. 2013;304(1):G48-56 DOI: 10.1152/ ajpgi.00317.2012.

18. Hulzebos CV, van Zoonen AG, Hulscher JB, Schat TE, Kooi EM, Koehorst M, Boverhof R, Krabbe PF, Groen AK, Verkade HJ. Fecal Bile Salts and the Development of Necrotizing Enterocolitis in Preterm Infants. PLoS One. 2017;12(1):e0168633 DOI: 10.1371/journal.pone.0168633.

19. Halpern MD, Holubec H, Dominguez JA, Meza YG, Williams CS, Ruth MC, McCuskey RS, Dvorak B. Hepatic inflammatory mediators contribute to intestinal damage in necrotizing enterocolitis. Am J Physiol Gastrointest Liver Physiol. 2003;284(4):G695-702 DOI: 10.1152/ajpgi.00353.2002.

20. Bouic PJ, Lamprecht JH. Plant sterols and sterolins: a review of their immune-modulating properties. Altern Med Rev. 1999;4(3):170-7.

21. Cheon JH, Kim JS, Kim JM, Kim N, Jung HC, Song IS. Plant sterol guggulsterone inhibits nuclear factor-kappaB signaling in intestinal epithelial cells by blocking IkappaB kinase and ameliorates acute murine colitis. Inflamm Bowel Dis. 2006;12(12):1152-61 DOI: 10.1097/01. mib.0000235830.94057.c6.

22. Ding Y, Nguyen HT, Kim Sl, Kim HW, Kim YH. The regulation of inflammatory cytokine secretion in macrophage cell line by the chemical constituents of Rhus sylvestris. Bioorg Med Chem Lett. 2009;19(13):3607-10 DOI: 10.1016/j.bmcl.2009.04.129.

23. Mencarelli A, Renga B, Palladino G, Distrutti E, Fiorucci S. The plant sterol guggulsterone attenuates inflammation and immune dysfunction in murine models of inflammatory bowel disease. Biochem Pharmacol. 2009;78(9):1214-23 DOI: 10.1016/j.bcp.2009.06.026.

24. Valerio $M$, Awad $A B$. beta-Sitosterol down-regulates some pro-inflammatory signal transduction pathways by increasing the activity of tyrosine phosphatase SHP-1 in J774A.1 murine macrophages. Int Immunopharmacol. 2011;11(8):1012-7 DOI: 10.1016/j.intimp.2011.02.018.

25. Valerio MS, Minderman H, Mace T, Awad AB. beta-Sitosterol modulates TLR4 receptor expression and intracellular MyD88-dependent pathway activation in J774A.1 murine macrophages. Cell Immunol. 2013;285(1-2):76-83 DOI: 10.1016/j.cellimm.2013.08.007.

26. Aldini R, Micucci M, Cevenini M, Fato R, Bergamini C, Nanni C, Cont M, Camborata C, Spinozzi S, Montagnani M, Roda G, D'Errico-Grigioni A, Rosini F, Roda A, Mazzella G, Chiarini A, Budriesi R. Antiinflammatory effect of phytosterols in experimental murine colitis model: prevention, induction, remission study. PLoS One. 2014;9(9):e108112 DOI: 10.1371/journal.pone.0108112.

27. Kim KA, Lee IA, Gu W, Hyam SR, Kim DH. beta-Sitosterol attenuates high-fat diet-induced intestinal inflammation in mice by inhibiting the binding of lipopolysaccharide to toll-like receptor 4 in the NFkappaB pathway. Mol Nutr Food Res. 2014;58(5):963-72 DOI: 10.1002/mnfr.201300433.

28. Plat J, Hendrikx T, Bieghs V, Jeurissen ML, Walenbergh SM, van Gorp PJ, De Smet E, Konings M, Vreugdenhil AC, Guichot YD, Rensen SS, Buurman WA, Greve JW, Lutjohann D, Mensink RP, ShiriSverdlov R. Protective role of plant sterol and stanol esters in liver inflammation: insights from mice and humans. PLoS One. 2014;9(10):e110758 DOI: 10.1371/journal.pone.0110758.

29. te Velde AA, Brull F, Heinsbroek SE, Meijer SL, Lutjohann D, Vreugdenhil A, Plat J. Effects of Dietary Plant Sterols and Stanol Esters with Low- and High-Fat Diets in Chronic and Acute Models for Experimental Colitis. Nutrients. 2015;7(10):8518-31 DOI: 10.3390/nu7105412.

30. van Gorp C, de Lange IH, Spiller OB, Dewez F, Cillero Pastor B, Heeren RMA, Kessels L, Kloosterboer N, van Gemert WG, Beeton ML, Stock SJ, Jobe AH, Payne MS, Kemp MW, Zimmermann LJ, Kramer BW, Plat J, Wolfs T. Protection of the Ovine Fetal Gut against Ureaplasma-Induced Chorioamnionitis: A Potential Role for Plant Sterols. Nutrients. 2019;11(5) DOI: 10.3390/nu11050968. 
31. Fekete E, Resch BA, Benedeczky I. Histochemical and ultrastructural features of the developing enteric nervous system of the human foetal small intestine. Histol Histopathol. 1995;10(1):127-34.

32. Fekete $E$, Bagyánszki M, Resch BA. Prenatal development of the myenteric plexus in the human fetal small intestine. Acta Biol Szeged [Internet]. 2000;44(1-4):3-19.

33. Tita AT, Andrews WW. Diagnosis and management of clinical chorioamnionitis. Clin Perinatol. 2010;37(2):339-54 DOI: 10.1016/j.clp.2010.02.003.

34. Bush TG. Enteric glial cells. An upstream target for induction of necrotizing enterocolitis and Crohn's disease? Bioessays. 2002;24(2):130-40 DOI: 10.1002/bies.10039.

35. De Giorgio R, Giancola F, Boschetti E, Abdo H, Lardeux B, Neunlist M. Enteric glia and neuroprotection: basic and clinical aspects. Am J Physiol Gastrointest Liver Physiol. 2012;303(8):G887-93 DOI: 10.1152/ajpgi.00096.2012.

36. Joseph NM, He S, Quintana E, Kim YG, Nunez G, Morrison SJ. Enteric glia are multipotent in culture but primarily form glia in the adult rodent gut. J Clin Invest. 2011;121(9):3398-411 DOI: 10.1172/ JCI58186.

37. Laranjeira C, Sandgren K, Kessaris N, Richardson W, Potocnik A, Vanden Berghe P, Pachnis V. Glial cells in the mouse enteric nervous system can undergo neurogenesis in response to injury. J Clin Invest. 2011;121(9):3412-24 DOI: 10.1172/jci58200.

38. Furness J. The Enteric Nervous System. Oxford, UK: Blackwell. 2006.

39. Vento M, Moro M, Escrig R, Arruza L, Villar G, Izquierdo I, Roberts LJ, 2nd, Arduini A, Escobar JJ, Sastre J, Asensi MA. Preterm resuscitation with low oxygen causes less oxidativestress, inflammation, and chronic lung disease. Pediatrics. 2009;124(3):e439-49 DOI: 10.1542/peds.2009-0434.

40. Bohrer B, Silveira RC, Neto EC, Procianoy RS. Mechanical ventilation of newborns infant changes in plasma pro- and anti-inflammatory cytokines. J Pediatr. 2010;156(1):16-9 DOI: 10.1016/j. jpeds.2009.07.027.

41. Lavoie PM, Lavoie JC, Watson C, Rouleau T, Chang BA, Chessex P. Inflammatory response in preterm infants is induced early in life by oxygen and modulated by total parenteral nutrition. Pediatr Res. 2010;68(3):248-51 DOI: 10.1203/PDR.0b013e3181eb2f18.

42. Stoll BJ, Hansen NI, Sanchez PJ, Faix RG, Poindexter BB, Van Meurs KP, Bizzarro MJ, Goldberg RN, Frantz ID, 3rd, Hale EC, Shankaran S, Kennedy K, Carlo WA, Watterberg KL, Bell EF, Walsh MC, Schibler K, Laptook AR, Shane AL, Schrag SJ, Das A, Higgins RD, Eunice Kennedy Shriver National Institute of Child H, Human Development Neonatal Research N. Early onset neonatal sepsis: the burden of group B Streptococcal and E. coli disease continues. Pediatrics. 2011;127(5):817-26 DOI: 10.1542/peds.2010-2217.

43. Leviton A, O'Shea TM, Bednarek FJ, Allred EN, Fichorova RN, Dammann O, Investigators ES. Systemic responses of preterm newborns with presumed or documented bacteraemia. Acta Paediatr. 2012;101(4):355-9 DOI: 10.1111/j.1651-2227.2011.02527.x.

44. Bose CL, Laughon MM, Allred EN, O'Shea TM, Van Marter LJ, Ehrenkranz RA, Fichorova RN, Leviton A, Investigators ES. Systemic inflammation associated with mechanical ventilation among extremely preterm infants. Cytokine. 2013;61(1):315-22 DOI: 10.1016/j.cyto.2012.10.014.

45. Joly A, Leulier F, De Vadder F. Microbial Modulation of the Development and Physiology of the Enteric Nervous System. Trends Microbiol. 2020 DOI: 10.1016/j.tim.2020.11.007.

46. Uesaka T, Young HM, Pachnis V, Enomoto H. Development of the intrinsic and extrinsic innervation of the gut. Dev Biol. 2016;417(2):158-67 DOI: 10.1016/j.ydbio.2016.04.016.

47. Chin AM, Hill DR, Aurora M, Spence JR. Morphogenesis and maturation of the embryonic and postnatal intestine. Semin Cell Dev Biol. 2017;66:81-93 DOI: 10.1016/j.semcdb.2017.01.011. 
48. Geboes K, Collins S. Structural abnormalities of the nervous system in Crohn's disease and ulcerative colitis. Neurogastroenterol Motil. 1998;10(3):189-202.

49. Wirthlin DJ, Cullen JJ, Spates ST, Conklin JL, Murray J, Caropreso DK, Ephgrave KS. Gastrointestinal transit during endotoxemia: the role of nitric oxide. J Surg Res. 1996;60(2):307-11 DOI: 10.1006/ jsre.1996.0048.

50. Hellstrom PM, al-Saffar A, Ljung T, Theodorsson E. Endotoxin actions on myoelectric activity, transit, and neuropeptides in the gut. Role of nitric oxide. Dig Dis Sci. 1997;42(8):1640-51 DOI: 10.1023/a:1018897028463.

51. Cullen JJ, Doty RC, Ephgrave KS, Hinkhouse MM, Broadhurst K. Changes in intestinal transit and absorption during endotoxemia are dose dependent. J Surg Res. 1999;81(1):81-6 DOI: 10.1006/ jsre.1998.5452.

52. Cullen JJ, Mercer D, Hinkhouse M, Ephgrave KS, Conklin JL. Effects of endotoxin on regulation of intestinal smooth muscle nitric oxide synthase and intestinal transit. Surgery. 1999;125(3):339-44.

53. Gale RP. Development of the immune system in human fetal liver. Thymus. 1987;10(1-2):45-56 DOI: 10.1007/978-94-009-3365-1_6.

54. Naito M, Hasegawa G, Takahashi K. Development, differentiation, and maturation of Kupffer cells. Microsc Res Tech. 1997;39(4):350-64 DOI: 10.1002/(SICI)1097-0029(19971115)39:4<350::AIDJEMT5>3.0.CO;2-L.

55. Naito M, Takahashi K, Nishikawa S. Development, differentiation, and maturation of macrophages in the fetal mouse liver. J Leukoc Biol. 1990;48(1):27-37 DOI: 10.1002/jlb.48.1.27.

56. Mebius RE, Miyamoto T, Christensen J, Domen J, Cupedo T, Weissman IL, Akashi K. The fetal liver counterpart of adult common lymphoid progenitors gives rise to all lymphoid lineages, CD45+CD4+CD3-cells, as well as macrophages. J Immunol. 2001;166(11):6593-601 DOI: 10.4049/ jimmunol.166.11.6593.

57. Naito M, Hasegawa G, Ebe $Y$, Yamamoto T. Differentiation and function of Kupffer cells. Med Electron Microsc. 2004;37(1):16-28 DOI: 10.1007/s00795-003-0228-x.

58. Sheth K, Bankey P. The liver as an immune organ. Curr Opin Crit Care. 2001;7(2):99-104 DOI: 10.1097/00075198-200104000-00008.

59. Gussenhoven R, Westerlaken RJJ, Ophelders D, Jobe AH, Kemp MW, Kallapur SG, Zimmermann LJ, Sangild PT, Pankratova S, Gressens P, Kramer BW, Fleiss B, Wolfs T. Chorioamnionitis, neuroinflammation, and injury: timing is key in the preterm ovine fetus. J Neuroinflammation. 2018;15(1):113 DOI: 10.1186/s12974-018-1149-x.

60. Zarate MA, Wesolowski SR, Nguyen LM, De Dios RK, Wilkening RB, Rozance PJ, Wright CJ. In utero inflammatory challenge induces an early activation of the hepatic innate immune response in late gestation fetalsheep. Innate Immun. 2020:1753425920928388DOI: 10.1177/1753425920928388.

61. Pfisterer C, Faber R, Horn LC. Chorioamnionitis-induced changes of fetal extramedullar hematopoiesis in the second trimester of gestation. Is diagnosis from fetal autopsy possible? Virchows Arch. 2005;446(2):150-6 DOI: 10.1007/s00428-004-1151-9.

62. Kim CH. Homeostatic and pathogenic extramedullary hematopoiesis. J Blood Med. 2010;1:13-9 DOI: $10.2147 / J B M . S 7224$.

63. Hegyi P, Maleth J, Walters JR, Hofmann AF, Keely SJ. Guts and Gall: Bile Acids in Regulation of Intestinal Epithelial Function in Health and Disease. Physiol Rev. 2018;98(4):1983-2023 DOI: 10.1152/physrev.00054.2017. 
64. Knapp S, Kehring A, Stepp J, Calton CM, Gephart SM, Bandlamuri S, Boyle KE, Dietz Gl, Johnson $\mathrm{H}$, Romo RE, Spencer M, Bedrick AD, Halpern MD. Elevated Coefficient of Variation in Total Fecal Bile Acids Precedes Diagnosis of Necrotizing Enterocolitis. Sci Rep. 2020;10(1):249 DOI: 10.1038/ s41598-019-57178-0.

65. Dobrenen H, Patrick SM, Khailová L, DvpYák B, Halpern M. T1877 Interleukin-18 Upregulates the Apical Sodium-Dependent Bile Acid Transporter (ASBT) in Neonatal Rat lleum and IEC-6 Cells. Gastroenterology. 2010;138.

66. Rogan DT. Overexpression of the Apical Sodium-Dependent Bile Acid Transporter to Replicate Necrotizing Enterocolitis in IEC-6 Cells. The University of Arizona.; 2012.

67. Gantert M, Been JV, Gavilanes AW, Garnier Y, Zimmermann LJ, Kramer BW. Chorioamnionitis: a multiorgan disease of the fetus? J Perinatol. 2010;30 Suppl:S21-30 DOI: 10.1038/jp.2010.96.

68. Kallapur SG, Nitsos I, Moss TJ, Polglase GR, Pillow JJ, Cheah FC, Kramer BW, Newnham JP, Ikegami $\mathrm{M}$, Jobe AH. IL-1 mediates pulmonary and systemic inflammatory responses to chorioamnionitis induced by lipopolysaccharide. Am J Respir Crit Care Med. 2009;179(10):955-61 DOI: 10.1164/ rccm.200811-17280C.

69. Wolfs TG, Kallapur SG, Knox CL, Thuijls G, Nitsos I, Polglase GR, Collins JJ, Kroon E, Spierings J, Shroyer NF, Newnham JP, Jobe AH, Kramer BW. Antenatal ureaplasma infection impairs development of the fetal ovine gut in an IL-1-dependent manner. Mucosal Immunol. 2013;6(3):54756 DOI: $10.1038 / \mathrm{mi} .2012 .97$.

70. Nikiforou M, Vanderlocht J, Chougnet CA, Jellema RK, Ophelders DR, Joosten M, Kloosterboer N, Senden-Gijsbers BL, Germeraad WT, Kramer BW, Wolfs TG. Prophylactic Interleukin-2 Treatment Prevents Fetal Gut Inflammation and Injury in an Ovine Model of Chorioamnionitis. Inflamm Bowel Dis. 2015;21(9):2026-38 DOI: 10.1097/MIB.0000000000000455.

71. Mellies MJ, Burton K, Larsen R, Fixler D, Glueck CJ. Cholesterol, phytosterols, and polyunsaturated/ saturated fatty acid ratios during the first 12 months of lactation. The American Journal of Clinical Nutrition. 1979;32(12):2383-9 DOI: 10.1093/ajcn/32.12.2383.

72. Laitinen K, Isolauri E, Kaipiainen L, Gylling H, Miettinen TA. Plant stanol ester spreads as components of a balanced diet for pregnant and breast-feeding women: evaluation of clinical safety. Br J Nutr. 2009;101(12):1797-804 DOI: 10.1017/S0007114508133608.

73. Hamdan IJA, Sanchez-Siles LM, Matencio E, Bermudez JD, Garcia-Llatas G, Lagarda MJ. Sterols in human milk during lactation: bioaccessibility and estimated intakes. Food Funct. 2018;9(12):656676 DOI: 10.1039/c8fo01795f.

74. Amaral C, Gallardo E, Rodrigues R, Pinto Leite R, Quelhas D, Tomaz C, Cardoso ML. Quantitative analysis of five sterols in amniotic fluid by GC-MS: application to the diagnosis of cholesterol biosynthesis defects. J Chromatogr B Analyt Technol Biomed Life Sci. 2010;878(23):2130-6 DOI: 10.1016/j.jchromb.2010.06.010.

75. Baardman ME, Erwich JJ, Berger RM, Hofstra RM, Kerstjens-Frederikse WS, Lutjohann D, Plosch T. The origin of fetal sterols in second-trimester amniotic fluid: endogenous synthesis or maternal-fetal transport? Am J Obstet Gynecol. 2012;207(3):202 e19-25 DOI: 10.1016/j.ajog.2012.06.003.

76. Moreno JJ. Effect of olive oil minor components on oxidative stress and arachidonic acid mobilization and metabolism by macrophages RAW 264.7. Free Radic Biol Med. 2003;35(9):107381 DOI: 10.1016/s0891-5849(03)00465-9.

77. Awad AB, Toczek J, Fink CS. Phytosterols decrease prostaglandin release in cultured P388D 1/MAB macrophages. Prostaglandins Leukot Essent Fatty Acids. 2004;70(6):511-20 DOI: 10.1016/j. plefa.2003.11.005. 
78. Vivancos M, Moreno JJ. beta-Sitosterol modulates antioxidant enzyme response in RAW 264.7 macrophages. Free Radic Biol Med. 2005;39(1):91-7 DOI: 10.1016/j.freeradbiomed.2005.02.025.

79. Vivancos M, Moreno JJ. Effect of resveratrol, tyrosol and beta-sitosterol on oxidised low-density lipoprotein-stimulated oxidative stress, arachidonic acid release and prostaglandin E2 synthesis by RAW 264.7 macrophages. Br J Nutr. 2008;99(6):1 199-207 DOI: 10.1017/S0007114507876203.

80. Clayton PT, Bowron A, Mills KA, Massoud A, Casteels M, Milla PJ. Phytosterolemia in children with parenteral nutrition-associated cholestatic liver disease. Gastroenterology. 1993;105(6):1806-13 DOI: 10.1016/0016-5085(93)91079-w.

81. El Kasmi KC, Anderson AL, Devereaux MW, Vue PM, Zhang W, Setchell KD, Karpen SJ, Sokol RJ. Phytosterols promote liver injury and Kupffer cell activation in parenteral nutrition-associated liver disease. Sci Transl Med. 2013;5(206):206ra137 DOI: 10.1126/scitransImed.3006898.

82. Mutanen A, Nissinen MJ, Lohi J, Heikkila P, Gylling H, Pakarinen MP. Serum plant sterols, cholestanol, and cholesterol precursors associate with histological liver injury in pediatric onset intestinal failure. Am J Clin Nutr. 2014;100(4):1085-94 DOI: 10.3945/ajcn.114.088781.

83. Mutanen A, Lohi J, Heikkila P, Jalanko H, Pakarinen MP. Liver Inflammation Relates to Decreased Canalicular Bile Transporter Expression in Pediatric Onset Intestinal Failure. Ann Surg. 2018;268(2):332-9 DOI: 10.1097/SLA.0000000000002187.

84. Khalaf RT, Sokol RJ. New Insights Into Intestinal Failure-Associated Liver Disease in Children. Hepatology. 2020;71(4):1486-98 DOI: 10.1002/hep.31152.

85. Atger VM, de la Llera Moya M, Stoudt GW, Rodrigueza WV, Phillips MC, Rothblat GH. Cyclodextrins as catalysts for the removal of cholesterol from macrophage foam cells. J Clin Invest. 1997;99(4):773-80 DOI: 10.1172/JCI119223.

86. Liu SM, Cogny A, Kockx M, Dean RT, Gaus K, Jessup W, Kritharides L. Cyclodextrins differentially mobilize free and esterified cholesterol from primary human foam cell macrophages. J Lipid Res. 2003;44(6):1156-66 DOI: 10.1194/jlr.M200464-JLR200.

87. Zimmer S, Grebe A, Bakke SS, Bode N, Halvorsen B, Ulas T, Skjelland M, De Nardo D, Labzin LI, Kerksiek A, Hempel C, Heneka MT, Hawxhurst V, Fitzgerald ML, Trebicka J, Bjorkhem I, Gustafsson JA, Westerterp M, Tall AR, Wright SD, Espevik T, Schultze JL, Nickenig G, Lutjohann D, Latz E. Cyclodextrin promotes atherosclerosis regression via macrophage reprogramming. Sci Transl Med. 2016;8(333):333ra50 DOI: 10.1126/scitransImed.aad6100.

88. Plat J, Nichols JA, Mensink RP. Plant sterols and stanols: effects on mixed micellar composition and LXR (target gene) activation. J Lipid Res. 2005;46(11):2468-76 DOI: 10.1194/jlr.M500272-JLR200.

89. Jakobsson T, Vedin LL, Hassan T, Venteclef N, Greco D, D'Amato M, Treuter E, Gustafsson JA, Steffensen KR. The oxysterol receptor LXRbeta protects against DSS- and TNBS-induced colitis in mice. Mucosal Immunol. 2014;7(6):1416-28 DOI: 10.1038/mi.2014.31.

90. Jablin MS, Flasinski M, Dubey M, Ratnaweera DR, Broniatowski M, Dynarowicz-Latka P, Majewski $\mathrm{J}$. Effects of beta-cyclodextrin on the structure of sphingomyelin/cholesterol model membranes. Biophys J. 2010;99(5):1475-81 DOI: 10.1016/j.bpj.2010.06.028.

91. Meister AL, Burkholder CR, Doheny KK, Travagli RA. Ghrelin ameliorates the phenotype of newborn rats induced with mild necrotizing enterocolitis. Neurogastroenterol Motil. 2019;31(11):e13682 DOI: $10.1111 / \mathrm{nmo} .13682$.

92. Bakke SS, Aune MH, Niyonzima N, Pilely K, Ryan L, Skjelland M, Garred P, Aukrust P, Halvorsen B, Latz E, Damas JK, Mollnes TE, Espevik T. Cyclodextrin Reduces Cholesterol Crystal-Induced Inflammation by Modulating Complement Activation. J Immunol. 2017;199(8):2910-20 DOI: 10.4049/jimmunol.1700302. 
93. Vlassaks E, Gavilanes AW, Bieghs V, Reinartz A, Gassler N, Van Gorp PJ, Gijbels MJ, Bekers O, Zimmermann LJ, Pillow JJ, Polglase GR, Nitsos I, Newnham JP, Kallapur SG, Jobe AH, ShiriSverdlov R, Kramer BW. Antenatal exposure to chorioamnionitis affects lipid metabolism in 7-week-old sheep. J Dev Orig Health Dis. 2012;3(2):103-10 DOI: 10.1017/S2040174412000049.

94. Ng PY, Ireland DJ, Keelan JA. Drugs to block cytokine signaling for the prevention and treatment of inflammation induced preterm birth. Frontiers in immunology. 2015;6:166.

95. Nikiforou M, Vanderlocht J, Chougnet CA, Jellema RK, Ophelders DR, Joosten M, Kloosterboer $\mathrm{N}$, Senden-Gijsbers BL, Germeraad WT, Kramer BW. Prophylactic interleukin-2 treatment prevents fetal gut inflammation and injury in an ovine model of chorioamnionitis. Inflammatory bowel diseases. 2015;21(9):2026-38.

96. Willems MG, Ophelders DR, Nikiforou M, Jellema RK, Butz A, Delhaas T, Kramer BW, Wolfs TG. Systemic interleukin-2 administration improves lung function and modulates chorioamnionitisinduced pulmonary inflammation in the ovine fetus. American Journal of Physiology-Lung Cellular and Molecular Physiology. 2015;310(1):L1-L7.

97. Keelan JA, Payne MS, Kemp MW, Ireland DJ, Newnham JP. A new, potent, and placenta-permeable macrolide antibiotic, solithromycin, for the prevention and treatment of bacterial infections in pregnancy. Frontiers in immunology. 2016;7:111.

98. Ophelders DR, Gussenhoven R, Lammens M, Küsters B, Kemp MW, Newnham JP, Payne MS, Kallapur SG, Jobe AH, Zimmermann LJ. Neuroinflammation and structural injury of the fetal ovine brain following intra-amniotic Candida albicans exposure. Journal of neuroinflammation. 2016;13(1):29.

99. Paton MC, Allison BJ, Li J, Fahey MC, Sutherland AE, Nitsos I, Bischof RJ, Dean JM, Moss TJ, Polglase GR. Human umbilical cord blood therapy protects cerebral white matter from systemic LPS exposure in preterm fetal sheep. Developmental Neuroscience. 2018;40(3):258-70.

100. Gomez-Lopez N, Romero R, Garcia-Flores V, Leng Y, Miller D, Hassan SS, Hsu C-D, Panaitescu B. Inhibition of the NLRP3 inflammasome can prevent sterile intra-amniotic inflammation, preterm labor/birth, and adverse neonatal outcomes. Biology of reproduction. 2019;100(5):1306-18.

101. van Gorp C, de Lange IH, Spiller OB, Dewez F, Cillero Pastor B, Heeren R, Kessels L, Kloosterboer N, van Gemert WG, Beeton ML. Protection of the Ovine Fetal Gut against Ureaplasma-Induced Chorioamnionitis: A Potential Role for Plant Sterols. Nutrients. 2019;11(5):968.

102. Galinsky R, Dhillon SK, Dean JM, Davidson JO, Lear CA, Wassink G, Nott F, Kelly SB, Fraser M, Yuill C. Tumor necrosis factor inhibition attenuates white matter gliosis after systemic inflammation in preterm fetal sheep. Journal of Neuroinflammation. 2020;17(1):1-16.

103. Redline RW. Inflammatory responses in the placenta and umbilical cord. Semin Fetal Neonatal Med. 2006;11(5):296-301 DOI: 10.1016/j.siny.2006.02.011.

104. Faye-Petersen OM. The placenta in preterm birth. J Clin Pathol. 2008;61(12):1261-75 DOI: 10.1136/ jcp.2008.055244.

105. Menon R, Taylor RN, Fortunato SJ. Chorioamnionitis--a complex pathophysiologic syndrome. Placenta. 2010;31(2):113-20 DOI: 10.1016/j.placenta.2009.11.012.

106. Witt RG, Blair L, Frascoli M, Rosen MJ, Nguyen QH, Bercovici S, Zompi S, Romero R, Mackenzie TC. Detection of microbial cell-free DNA in maternal and umbilical cord plasma in patients with chorioamnionitis using next generation sequencing. PLoS One. 2020;15(4):e0231239 DOI: 10.1371/journal.pone.0231239.

107. Boots AW, Bos LD, van der Schee MP, van Schooten F-J, Sterk PJ. Exhaled molecular fingerprinting in diagnosis and monitoring: validating volatile promises. Trends in molecular medicine. 2015;21(10):633-44. 
108. Ophelders DRMG, Boots AW, Hütten MC, Al-Nasiry S, Jellema RK, Spiller OB, van Schooten F-J, Smolinska A, Wolfs TGAM. Screening of Chorioamnionitis Using Volatile Organic Compound Detection in Exhaled Breath: A Pre-clinical Proof of Concept Study. Frontiers in Pediatrics. 2021;9(488) DOI: 10.3389/fped.2021.617906. 

Summary

Samenvatting 



\section{Summary}

Chorioamnionitis, inflammation of the fetal membranes during pregnancy, is, regardless of preterm birth, an independent risk factor for adverse neonatal outcomes including necrotizing enterocolitis (NEC). At present, the multifactorial pathogenesis of NEC is still incompletely understood. The involvement of chorioamnionitis in the induction of adverse intestinal and hepatic outcomes, combined with the presence of enteric nervous system (ENS) and enterohepatic circulation (EHC) abnormalities in NEC, prompted us to study the origin of these abnormalities. Therefore, the aim of this thesis was to investigate the effect of an antenatal intra-uterine infection on the ENS, liver and EHC of the fetus in order to gain essential insight how chorioamnionitis can lead to unfavorable gut and liver outcomes including NEC. Moreover, we aimed to investigate the consequences of perinatal stress on ENS and intestinal motility after birth. In addition, we investigated the potential of prophylactic intra-amniotic (IA) plant sterol administration to prevent chorioamnionitis-induced liver inflammation and potential EHC alterations, as plant sterols have been shown to have immune-modulatory effects in the healthy and inflamed intestine and liver.

Chorioamnionitis can be acute or chronic and is often polymicrobial. Therefore, we studied in chapter 2 the effects of acute, chronic and repetitive IA microbial exposure on the ileal mucosa, submucosa and ENS of premature lambs. We found that an acute inflammatory stimulus with lipopolysaccharide (LPS) or a chronic inflammatory stimulus with Ureaplasma parvum (UP) caused intestinal injury and inflammation in the mucosal and submucosal layers of the gut. Combined exposure of LPS and UP did not aggravate injury of the terminal ileum. Most importantly, chronic UP infection caused structural ENS alterations characterized by PGP9.5 and S100 $\beta$ immunoreactivity loss, implying neuronal and glial cell loss. Whether the observed ENS alterations result in functional abnormalities after birth remains to be elucidated. However, the observed changes in utero correspond with findings in neonates with NEC, underscoring the concept that NEC pathophysiology may originate already in utero, especially when elicited during the vulnerable second trimester.

In chapter 3, the time-dependent effects of 5 hours to 15 days of IA LPS exposure prior to premature delivery on the ileal mucosa, submucosa and ENS of premature sheep was investigated. Submucosal intestinal inflammation was detected after 4 days of IA LPS exposure and coincided with gut mucosal and fetal systemic inflammation. At the same time point, loss of PGP9.5 and S100 $\beta$ immunoreactivity in the myenteric plexus was observed, implying loss of neurons and glial cells, similar to the observations in chapter 2. Although initial ENS damage seemed to recover after prolonged IA LPS exposure, additional postnatal inflammatory hits likely to be encountered by a prematurely born child, may further harm the ENS and influence functional outcomes. In this context, 4 to 8 days after the start of an intra-uterine infection may be a window of increased ENS vulnerability, indicating that therapeutic interventions should ideally start before or at this time point. 
In chapter 4, we evaluated postnatal ENS and intestinal motility alterations in a NEC mouse model to investigate the consequences of perinatal stress after birth. We demonstrated that subjection to an experimental NEC-like injury protocol was associated with an increased NeuN and $\mathrm{S} 100 \beta$ immunoreactivity, implying an increase in the number of mature neurons and glial cells. Moreover, this was associated with an increased synaptophysin immunoreactivity (implying an increased presence of presynaptic vesicles in the submucosal and myenteric plexus) and time-dependent changes in intestinal motility. As increased numbers of mature neurons, glial cells and presynaptic vesicles cannot fully explain the observed changes in motility, additional studies are needed to unravel the mechanisms underlying these functional changes. Our findings are not in accordance with other studies on ENS abnormalities in NEC, both in human specimen and rodent NEC models, in which a loss of both glial cells and neurons was reported. Therefore the ENS changes in this NEC mouse model seem to represent a relatively milder disease state within the clinical NEC spectrum.

In chapter 5, we investigated the time-dependent effects of 5 hours to 15 days of intrauterine LPS exposure prior to premature delivery on the liver and EHC of premature sheep. We discovered that, in utero, the liver might be one of the first organs to induce a systemic innate immune response by early cytokine production as a result of chorioamnionitis. Altered fetal hepatic erythropoiesis as a reaction to inflammation was detected at day 8 and day 15 after IA LPS administration that might be the result of hepatic cytokine production, ileal inflammation and ongoing fetal inflammatory response syndrome (FIRS). This ongoing fetal systemic and liver inflammation likely contributed to the decreased expression of several hepatic bile acids (BAs) transporters and resultant increased plasma BA levels that were observed 12 hours after IA LPS administration. Initial alterations to the EHC as a consequence of IA LPS exposure normalized over time. However, since a premature born child is likely to encounter additional postnatal inflammatory hits that might induce (additional) hepatic injury and/or EHC alterations, this chapter highlights that the liver must be considered in future perinatal research projects and ultimately in neonatal care of preterm infants that suffered from inflammatory stress. Importantly, additional inflammation may have further impact on the liver and EHC and as a consequence the host in general.

In chapter 6, we investigated whether an IA UP infection affected the liver and EHC. Next, the effects of prophylactic IA administration of a plant sterol mixture dissolved in sterol carrier $\beta$-cyclodextrin was evaluated in this context, as plant sterols are a promising intervention to prevent NEC development considering their postulated anti-inflammatory properties. We showed that IA UP exposure caused an inflammatory reaction in the liver, which was partially prevented by IA $\beta$-cyclodextrin administration. In addition, IA administration of $\beta$-cyclodextrin prior to UP caused changes in the expression of several hepatic BAs transporters, without influencing other aspects of the EHC. Surprisingly, addition of plant sterols to the carrier $\beta$-cyclodextrin did not have additional effects. Taken together, the work described in this chapter reveals that interventions in utero have the potential to improve the outcome of preterm infants with a suboptimal start in life. 


\section{Samenvatting}

Chorioamnionitis (onsteking van de vruchtvliezen ten gevolge van een infectie in de baarmoeder) is een veel voorkomende oorzaak van vroeggeboorte. Tevens is chorioamnionitis een onafhankelijke risicofactor voor problemen in het latere leven, waaronder necrotiserende enterocolitis (NEC). Bij NEC is er sprake van een ernstige darmontsteking en deze ziekte is één van de meest voorkomende acute gastro-intestinale aandoeningen op de neonatale intensive care. De multifactoriële pathogenese van NEC is helaas nog niet volledig bekend. De betrokkenheid van chorioamnionitis bij het induceren van negatieve darm- en leveruitkomsten, gecombineerd met de aanwezigheid van afwijkingen van het enterisch zenuwstelsel (ENS) en de enterohepatische kringloop (EHC) bij NEC, bracht ons ertoe de oorsprong van deze afwijkingen te bestuderen. Het doel van dit proefschrift was daarom het effect van een intra-uteriene infectie op het ENS, de lever en de EHC van de foetus te onderzoeken, om zo inzicht te krijgen in hoe chorioamnionitis kan leiden tot slechte darm- en leveruitkomsten, waaronder NEC. Daarnaast hebben we de gevolgen van perinatale stress op het ENS en darmmotiliteit na de geboorte onderzocht. Tevens onderzochten we of het profylactisch verrijken van het vruchtwater met plantensterolen leverontsteking en potentiële EHC-veranderingen ten gevolge van chorioamnionitis kan voorkomen, omdat eerder onderzoek aantoonde dat plantensterolen een ontstekingsreactie in de darm en lever gunstig kunnen beïnvloeden.

Chorioamnionitis kan zowel acuut als chronisch zijn en wordt vaak veroorzaakt door meerdere micro-organismen. Daarom hebben we in hoofdstuk $\mathbf{2}$ de effecten van acute, chronische en herhaalde microbiële blootstelling in de baarmoeder (IA) op de mucosa, submucosa en het ENS van het ileum van premature lammeren onderzocht. We ontdekten dat acute IA blootstelling aan de bacteriële toxine lipopolysaccharide (LPS) of een chronische blootstelling aan Ureaplasma parvum (UP) weefselbeschadiging en -ontsteking veroorzaakten in de mucosale en submucosale lagen van de darm. Gecombineerde blootstelling aan LPS en UP verergerde deze weefselschade niet. De meest belangrijke bevinding was dat een chronische UP-infectie structurele ENSveranderingen veroorzaakte die werden gekenmerkt door verlies van PGP9.5- en S100ßimmunoreactiviteit, wat verlies van neuronen en gliacellen impliceert. Of de waargenomen ENS-veranderingen leiden tot functionele afwijkingen na de geboorte moet nog worden onderzocht. De gevonden veranderingen in utero komen echter overeen met bevindingen bij pasgeborenen met NEC, hetgeen onderstreept dat de pathofysiologie van NEC al in utero kan ontstaan, vooral wanneer deze wordt uitgelokt tijdens het kwetsbare tweede trimester van de zwangerschap.

In hoofdstuk 3 werden de tijdsafhankelijke gevolgen van 5 uur tot 15 dagen IA blootstelling aan LPS voorafgaand aan vroeggeboorte, op de mucosa, submucosa en het ENS van het ileum van premature lammeren onderzocht. Vier dagen na blootstelling aan IA LPS werd ontsteking van zowel de mucosa als de submucosa van het ileum gedetecteerd, alsmede een systemische ontstekingsreactie van de foetus. Op hetzelfde tijdspunt werd een afname van de eiwitten PGP9.5 en S100 $\beta$ in de myenterische plexus waargenomen, hetgeen verlies van neuronen en gliacellen impliceert, vergelijkbaar met de waarnemingen in hoofdstuk 2. De initiële door IA LPS veroorzaakte ENS-schade leek 
na verloop van tijd te herstellen. Desalniettemin kunnen bijkomende postnatale infecties en andere vormen van onstekingsstress, die een te vroeg geboren kind waarschijnlijk zal doormaken, het ENS verder beschadigen en de ENS functies beïnvloeden. Onze bevindingen suggereren dat het ENS extra kwetsbaar is in de periode van 4 tot 8 dagen na het oplopen van een intra-uteriene infectie, hetgeen impliceert dat therapeutische interventies idealiter vóór of op dit tijdstip zouden moeten aanvangen.

In hoofdstuk 4 onderzochten we in een NEC-muismodel of er postnatale veranderingen optraden in het ENS en in de darmmotiliteit, om de gevolgen van perinatale ontstekingsstress na de geboorte te achterhalen. Wij hebben een verhoogde expressie van de eiwitten NeuN en $\mathrm{S} 100 \beta$ aangetoond in muizen die blootgesteld werden aan een experimenteel NEC-protocol, hetgeen duidt op een toename van het aantal mature neuronen en gliacellen. Bovendien ging dit gepaard met een verhoging van synaptophysine (hetgeen duidt op een verhoogde aanwezigheid van presynaptische blaasjes in de submucosale en myenterische plexus) en tijdsafhankelijke veranderingen in de darmmotiliteit. Aangezien het verhoogde aantal mature neuronen, gliacellen en presynaptische blaasjes de veranderingen in de darmmotiliteit niet volledig kunnen verklaren, zijn aanvullende studies nodig om de mechanismen die ten grondslag liggen aan deze functionele veranderingen te ontrafelen. Onze bevindingen komen niet overeen met eerdere studies naar ENS-veranderingen in NEC, zowel in weefsels afkomstig van neonaten met NEC, als in NEC-knaagdiermodellen, waarin een verlies van zowel gliacellen als neuronen werd beschreven. Daarom lijken de ENS-veranderingen in ons NEC-muismodel een relatief milder ziektebeloop binnen het klinische NEC-spectrum te weerspiegelen.

In hoofdstuk 5 werden de gevolgen van een IA blootstelling aan LPS op de lever en EHC van premature lammeren gedurende de periode van 5 uur tot 15 dagen in kaart gebracht. We ontdekten dat de lever van de foetus in de baarmoeder één van de eerste organen zou kunnen zijn die een foetale systemische ontsteking opwekt door vroegtijdige productie van cytokines als gevolg van chorioamnionitis. Op dag 8 en dag 15 na blootstelling aan IA LPS werden er, als reactie op ontsteking, veranderingen in de foetale hepatische erytropoëse gevonden. Dit zou het gevolg kunnen zijn van cytokineproductie door de lever, ontsteking van het ileum en de aanhoudende systemische immuunactivatie van de foetus (FIRS). Deze FIRS en leverontsteking hebben waarschijnlijk bijgedragen aan de verminderde expressie van verschillende galzouttransporters in de lever en de daardoor verhoogde plasma galzout spiegels die 12 uur na IA LPS toediening werden waargenomen. Aanvankelijke veranderingen van de EHC als gevolg van IA LPS blootstelling normaliseerden na verloop van tijd. Echter is er een aanzienlijk risico dat een te vroeg geboren kind na geboorte bijkomende postnatale infecties zal doormaken of blootgesteld wordt aan andere vormen van ontstekingsstress die (extra) leverbeschadiging en/of EHC-veranderingen kunnen veroorzaken. Deze gecombineerde feiten benadrukken dat de lever nader bestudeerd dient te worden in toekomstige perinatale onderzoeksprojecten en meegenomen dient te worden in de neonatale zorg van te vroeg geboren kinderen die een perinatale infectie hebben doorgemaakt. Belangrijk te vermelden is dat een bijkomende ontsteking verdere nadelige effecten kan hebben op de lever en $\mathrm{EHC}$ en als gevolg daarvan op de gezondheid van het kind in het algemeen. 
In hoofdstuk 6 hebben we het effect van een IA UP infectie op de lever en EHC onderzocht. Vervolgens werd gekeken of een profylactische IA toediening van een plantensterolmengsel opgelost in $\beta$-cyclodextrine een gunstig effect heeft in dit kader, aangezien plantensterolen een veelbelovende interventie zijn om de ontwikkeling van NEC te voorkomen gezien de eerder beschreven ontstekingsremmende eigenschappen. Deze studie toonde aan dat IA blootstelling aan UP een ontstekingsreactie in de lever veroorzaakte, die gedeeltelijk voorkomen werd door IA toediening van een plantensterolmengsel opgelost in $\beta$-cyclodextrine. Daarnaast veroorzaakte IA toediening van plantensterolen/ $\beta$-cyclodextrine voorafgaand aan UP-blootstelling veranderingen in de expressie van verschillende galzouttransporters in de lever, zonder andere aspecten van de EHC te beïnvloeden. Verrassend genoeg waren de farmacologische effecten van de plantensterolen opgelost in $\beta$-cyclodextrine gelijk aan de effecten van enkel het oplosmiddel $\beta$-cyclodextrine. Het onderzoek dat in dit hoofdstuk wordt beschreven laat zien dat interventies in utero de potentie hebben om de gezondheidsuitkomst van te vroeg geboren kinderen, met een suboptimale start in het leven, te verbeteren. 



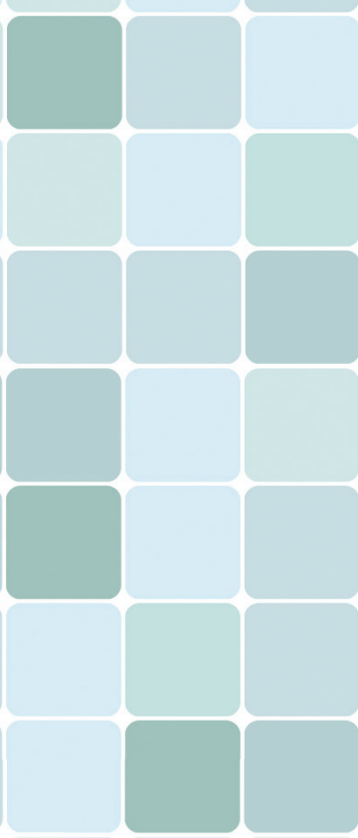

Impact 



\section{Impact}

\section{Relevance}

Preterm birth is a common and major worldwide health issue, contributing to significant neonatal morbidity and mortality. Around one in every ten births are preterm, accounting for approximately 15 million premature newborns each year. Due to complications, over one million premature newborns die shortly after birth.

Survivors of preterm birth have higher rates of adverse long-term health outcomes including cerebral palsy, sensory deficits, learning disabilities, altered intestinal motility and respiratory illnesses, compared with children born at term. The morbidity associated with preterm birth often extends to later in life and have a severe impact on emotional and financial status of patients, their families, caregivers and society.

Spontaneous preterm birth is in up to $40 \%$ associated with chorioamnionitis, which is an inflammation of the amniotic membranes. Chorioamnionitis typically results from an ascending bacterial infection from the lower genital tract (cervix and vagina), resulting in microbial colonization of the amniotic membranes, amniotic fluid, and/or umbilical cord. Most frequently, chorioamnionitis is asymptomatic, meaning that pregnant women do not have clinical symptoms and the presence of chorioamnionitis is recognized after delivery.

Chorioamnionitis can also, independently of preterm birth, provoke postnatal adverse effects, of which necrotizing enterocolitis (NEC) is one of the most severe neonatal gastrointestinal emergencies that predominantly affects premature infants. NEC is an inflammatory intestinal disorder, characterized by variable damage to the intestinal tract. The natural history of NEC is a fulminant progression with severe inflammation of the bowel, ischemia and necrosis, leading to septic shock, multiple organ failure, and death within hours or days of onset if appropriate treatment is not timely instituted. Mortality generally ranges from 15 to $30 \%$, but increases up to $50 \%$ for extremely low birth weight children treated surgically. Infants that do survive are at increased risk for e.g. intestinal failure, feeding difficulties, growth retardation and developmental delay as they age.

Despite advancing medical care, NEC treatment remains largely symptomatic. Moreover, effective preventative strategies that reduce the prevalence of NEC are lacking. These can only be achieved with a more profound understanding of the pathophysiological mechanisms leading to NEC, since the multifactorial pathogenesis of NEC is still incompletely understood.

\section{Innovation}

Firstly, the findings described in this thesis support the concept that intestinal neuropathological lesions found in NEC (loss of enteric neurons and glial cells in the submucosal and myenteric plexus) have their origin far before NEC onset and these enteric nervous system (ENS) alterations are considered to result in dysfunctional intestinal motility facilitating intraluminal bacterial overgrowth and translocation, therewith promoting the multi-factorial pathophysiology of NEC. 
Secondly, the data described in this thesis in combination with observations in literature, suggest that the enterohepatic circulation (EHC) alterations (elevated ileal bile acids (BAs) and altered expression of several BAs transporters) and liver inflammation found in NEC have their origin far before the onset of NEC and that both may result in ileal damage contributing to the multi-factorial pathophysiology of NEC.

To the best of our knowledge, we are the first describing the effects of chorioamnionitis on the ENS and EHC and postulate that chorioamnionitis-induced ENS and EHC alterations and liver inflammation may contribute to development of NEC. Moreover, we showed that the vulnerability of the fetus to injurious exposure during intra-uterine development is largest when exposed early in pregnancy, namely in the second trimester, potentially predisposing to NEC.

Ideally, one would like to start therapeutic interventions already in utero to prevent intestinal injury later in life and prevent NEC development. In this context, the work in this thesis showed that cyclodextrins, which are highly water-soluble, "ready-made," and commercially available, target liver inflammation and thereby potentially prevent adverse outcomes. Future studies should focus on the correct timing and dose of $\beta$-cyclodextrin administration and potential other interventions in utero during chorioamnionitis.

All together, the findings in this thesis demonstrate that chorioamnionitis can induce ENS and EHC alterations and liver inflammation which can adversely affect the fetal gut and liver, potentially predisposing to NEC. This increases our understanding of the multifactorial pathophysiology of NEC, which eventually will lead to therapeutic options and also preventative strategies.

\section{Target audience and future activities}

The findings in this thesis could be of interest to a wide audience. Firstly, the results are of significant interest for researchers, who could extend this research to further unravel the detrimental effects of chorioamnionitis and the multifactorial pathophysiology of NEC. Moreover, the anti-inflammatory effect of $\beta$-cyclodextrin can form the basis for scientists to further investigate the therapeutic effect of $\beta$-cyclodextrin in other organs, both in vulnerable neonates but also outside the pediatric setting. Moreover, the potential synergy with other pharmacological interventions should be explored. The described characterization of the in utero models facilitate the design of future studies where therapeutic strategies for gut- and liver-associated complications after chorioamnionitis can be tested.

Secondly, the results of this thesis can also be of interest for various clinicians including e.g. neonatologists, pediatricians, perinatologists and gynecologists who are interested in understanding the basic mechanisms underlying liver and gastrointestinal diseases in the newborn.

In conclusion, the studies described in this thesis contribute to the understanding of the effect of chorioamnionitis on the fetus and the multifactorial pathophysiology of NEC. In addition, this thesis presents a potential intervention to protect the fetal liver and gut in 
the context of chorioamnionitis. Nevertheless, additional research is required for clinical implementation and our translational ovine model is suitable to test the efficacy and feasibility of more comprehensive therapeutic strategies to prevent and/or treat liver and gastrointestinal complications after chorioamnionitis. 
Appendices 

Abbreviations 



\section{Abbreviations}

- 5-HT: Serotonin

- AF: Amniotic fluid

- AU: Arbitrary unit

- ASBT: Apical sodium-dependent bile acid transporter

- BA: Bile acid

- BSA: Bovine serum albumin

- BSEP: Bile salt export pump

- CA: Cholic acid

- CCU: Color-changing units

- CD3: Cluster of differentiation 3

- CDCA: chenodeoxycholic acid

- CHAT: Choline acetyltransferase

- CNS: Central nervous system

- CRP: C-reactive protein

- CYP7A1: Cholesterol 7alphahydroxylase

- CYP27A1: Cytochrome P450 family 27 subfamily A member 1

- DCA: Deoxycholic acid

- EHC: Enterohepatic circulation

- ENS: Enteric nervous system

- FCS: Fetal calf serum

- FGF19: Fibroblast growth factor 19
- FIRS: Fetal inflammatory response syndrome

- FXR: Farnesoid X receptor

- GAPDH: Glyceraldehyde 3-phosphate dehydrogenase

- GDNF: Glial-cell line derived neurotrophic factor

- GFAP: Glial fibrillary acidic protein

- Gl: Gastro-intestinal

- GpBAR1 (or TGR5): G-protein-coupled bile acid receptor 1

- H\&E: Hematoxylin and Eosin

- HELLP: Hemolysis, elevated liver enzymes and low platelets

- IA:Intra-amniotic

- IBA1: lonized calcium binding adaptor molecule 1

- IBABP: lleal bile acid-binding protein

- IBD: Inflammatory bowel disease

- IFALD: Intestinal failure-associated liver disease

- IL: Interleukin

- IPANs: Intrinsic primary afferent neurons

- IRAK3: Interleukin-1 receptor-associated kinase 3

- IUGR: Intra-uterine growth retardation 
- IQR: Interquartile range

- LCA: Lithocholic acid

- LPS: Lipopolysaccharide

- LXR: Liver-X-receptor

- MPO: Myeloperoxidase

- MMP: Matrix metalloproteinase

- NASH: Non-alcoholic fatty liver disease

- NEC: Necrotizing enterocolitis

- NeuN: Neuronal nuclei

- NGS: Normal goat serum

- NICU: Neonatal intensive care unit

- nNOS: Neuronal nitric oxide synthase

- NTCP: $\mathrm{Na}^{+}$-taurocholate cotransporting polypeptide

- OSTa: Organic solute transporter alpha

- OST $\beta$ : Organic solute transporter beta

- PBS: Phosphorylated buffer saline

- PNS: Peripheral nervous system

- PGP9.5: Protein gene product 9.5

- PPIA: Peptidylprolyl isomerase A

- PPROM: Preterm premature rupture of the membranes

- qPCR: Quantitative real-time polymerase chain reaction
- RhlL-1 ra: Recombinant human IL-1 receptor antagonist

- RPS15: Ribosomal protein S15

- Spp: Species

- tBAs: Total bile acids

- TCA:Taurocholic acid

- TCDCA:Taurochenodeoxycholic acid

- TDCA:Taurodeoxycholic acid

- TLR:Toll-like receptor

- TNF-a: Tumor necrosis factor alpha

- UP: Ureaplasma parvum

- VIP: Vasoactive intestinal polypeptide

- VOCs: volatile organic compounds 


Dankwoord 



\section{Dankwoord}

Last but not least: het dankwoord, waarschijnlijk het meest gelezen hoofdstuk van dit proefschrift. Een hoofdstuk waarbij ik graag even stil wil staan bij mijn promotietraject en alle leuke en lieve mensen die ik hierbij heb mogen ontmoeten, die mij hebben ondersteund, geholpen en goede raad hebben gegeven.

Wat heb ik veel geleerd in de afgelopen vijf jaar. Veel over wetenschap natuurlijk, maar ook heb ik mijzelf beter leren kennen. Mijn promotie was een ontzettend leuke, bijzondere en leerzame periode in mijn leven. Daarentegen zijn ook mij de bijbehorende bloed, zweet en tranen niet bespaard gebleven. Terugkijkend had ik deze tijd voor geen goud willen missen!

Dit proefschrift is tot stand gekomen met de hulp en steun van geweldige collega's, familie en vrienden. Zonder jullie was dit proefschrift niet geworden wat het nu is. Mijn dank is groot en bijzonder gericht aan onderstaande personen.

Prof. Dr. van Gemert, Wim, mijn promoter, dank dat je mij de kans hebt gegeven om dit promotietraject te doen. Jouw enthousiasme is aanstekelijk en tijdens meetings kwam jij dan ook altijd met heel veel nieuwe ideeën en/of samenwerkingen met andere specialismen. Dit nadat er eerst uitgebreid werd gekletst en gelachen over van alles en nog wat. Nou ja, eigenlijk was jij voornamelijk aan het kletsen en met een grote glimlach luisterde ik altijd graag naar al jouw verhalen. Tevens was je altijd in voor een grapje en kon ik goed met je sparren over bijvoorbeeld mijn toekomst en mogelijke specialisaties.

Waar ik moeilijkheden zag, zei jij altijd dat het wel goed zou komen. En je hebt gelijk gehad, het is goedgekomen, met dit mooie proefschrift als resultaat! Ik heb enorm veel van je geleerd en ik wil je ontzettend bedanken voor de fijne begeleiding en het vertrouwen, de vrijheid en de kansen die je me hebt gegeven. Gelukkig blijven we nog vaak samenwerken op de OK, welliswaar ieder aan de andere kant van het doek.

Tim Wolfs, mijn promotor, je hebt me gered van de darmslices en daarvoor ben ik je eeuwig dankbaar! Je hielp me aan een nieuw onderwerp, waardoor uiteindelijk dit mooie proefschrift is ontstaan. Een proefschrift waarbij we een mooie samenwerking tussen de kindergeneeskunde en de chirugie tot stand hebben gebracht.

Wanneer ik door de bomen het bos niet meer zag (door de vele data uitkomsten), hielp jij mij om hier weer lijn in te krijgen. Tevens heb je me geleerd om deze data op een goede wetenschappelijke manier te analyseren, te beoordelen en te beschrijven. Mede door jouw goede begeleiding en kritische blik hebben we het onderzoek naar een hoger niveau getild en is hier een aantal mooie publicaties uit voortgekomen.

Tijdens de vele brainstorm en feedback sessies (en daarbuiten) viel er altijd met jou wat te geinen. Toch jammer dat toen ik tijdens het skireisje op het punt stond om in de sneeuw te springen (om de beste deal uit mijn leven te kunnen sluiten!), jij toch angst kreeg voor je autobekleding. Tim, nogmaals enorm bedankt voor alles! 
Kaatje Lenaerts, mijn co-promotor, iedere week zaten wij samen om de voortgang van het onderzoek te bespreken en problemen in het lab te tackelen. Jij had altijd weer een oplossing wanneer het onderzoek in het lab vast liep of een nieuw idee om een wetenschappelijk vraagstuk verder uit te zoeken en meer diepgang te geven. Daarnaast had je altijd tijd voor mij als ik tussendoor weer vragen had en hielp je me verder met uiteenlopende zaken van statistiek tot schrijftechnische vaardigheden.

Wij bleken echt een top sollicitatieteam te zijn! Samen wisten we altijd de beste studenten eruit te pikken die mij konden helpen met mijn projecten. Ik wil je ontzettend graag bedanken voor de fijne begeleiding, de fijne gesprekken en alles wat jij mij hebt geleerd. Tevens mijn dank dat je me in contact bracht met jouw partner, Frank Mooren, die deze supergave omslag heeft ontworpen!

Graag wil ik de leden van de beoordelingscommissie van dit proefschrift, bestaande uit: Prof. Dr. Olde Damink, Prof. Dr. Hulscher, Prof. Dr. Jonkers, Prof. Dr. Stassen en Dr. Derikx bedanken voor de deelname aan deze commissie en dank voor de tijd en moeite die jullie hebben genomen om mijn proefschrift kritisch te beoordelen.

Specifiek wil ik graag Prof. Dr. Steven Olde Damink bedanken voor het gebruik kunnen maken van de faciliteiten van het lab van de chirurgie. In het begin van mijn promotietraject was jij mijn surrogaat promotor en hebben we veel samen gezeten, waarbij jij in meetings altijd met ontzettend veel nieuwe onderzoeksideeën kwam. Helaas is het darmslicesproject niet van de grond gekomen zoals we hadden gehoopt. Ontzettend leuk dat je nu weer betrokken bent bij mijn promotie als voorzitter van de beoordelingscommissie.

Prof. Dr. Boris Kramer, toen het darmslicesproject strandde stond u meteen voor mij klaar om mij te helpen aan een nieuwe onderzoekslijn. De lever is uiteindelijk inderdaad een deel van het proefschrift geworden. Mijn dank hiervoor.

Matthias Hütten, zo fijn dat ik van jou het onderzoek naar het enteric nervous system (ENS) in het UP-LPS model af mocht ronden. Deze voorzet was een fijne start om aan het nieuwe onderwerp van mijn promotie te beginnen. Dank hiervoor, ook voor je betrokkenheid bij de studie, de hulp bij de statistiek en de gezelligheid tijdens de kindergeneeskunde uitjes.

Marcel den Dulk, dank dat je tijd vrij maakte om meermaals met mij te brainstormen over de leverstudies en samen met mij in de literatuur te duiken. Tevens dank voor de kritische blik waarmee je de manuscripten nakeek. Jouw manier om statistiek uit te leggen, als "het bakken van taarten", was een echte eyeopener. Ook wij zullen elkaar vaker blijven zien aan de andere kant van het doek.

Miriam Habex-Froidmont, heel erg bedankt voor je ondersteuning gedurende mijn promotietraject.

Annemarie, Bas, Cathy (mede hondenmama!), Chantal, Hans en Mo, bedankt voor alle hulp, adviezen en het aanleren van verschillende technieken in het lab. Bas, de bedenker 
van de confetti-techniek, wat hebben wij onwijs geknutseld aan de darmslices/confetti's. Klinkt vrolijk die confetti, de uitkomst minder. Gelukkig maakte al dat geinen met jou alles beter! Chantal, jij hebt mij zoveel werk uit handen genomen. Zonder jou was ik nog niet zo ver geweest, dank hiervoor! Mo, jij bent betrokken geweest bij al mijn studies, gaf jouw inbreng tijdens onze wekelijkse labmeeting met Kaatje en hebt me ontzettend veel in het lab geleerd en geholpen. Op ICT gebied heb je me ook de nodige keren uit de brand geholpen. Heel erg bedankt voor alles, anders had ik het nooit gehaald!

Kimberly, Lilian, Nico en Teun, bedankt voor alle hulp, adviezen en het aanleren van verschillende technieken in het lab. Kimberly, een van de weinige personen die "Noot Noot" (Pingu) tot haar skills kan rekenen. Dit was dan ook standaard onze begroeting. Heerlijk om met jou te kunnen kletsen over de gebeurtenissen in de slechtste programma's die te zien waren op de Nederlandse televisie. Lilian, bewonderingswaardig hoe jij alle kleuringen aan de praat hebt weten te krijgen. We hebben hierdoor een heel mooi scala aan ENS kleuringen kunnen doen, dank hiervoor! Als ik nog eens een keer een splinter in mijn vinger heb, weet ik je te vinden. Nico, jij bent echt een manus van alles. Voor iedere vraag had je wel een oplossing, wist je waar ik iets kon vinden, iets stond of bij wie ik moest zijn. Ook op ICT gebied heb je me geholpen aan de nodige belangrijke programma's. Daarnaast heb ik bewondering voor jouw operatie skills. Dank voor alles! Teun, lieve Teun, niemand zal jou en je bulderende lach ooit vergeten.

Lara Heij, dank voor de beoordeling van alle levercoupes. Deze data heeft gezorgd voor een doorbraak in de leverstudies en heeft daarmee deze studies tot een hoger niveau gebracht.

Alle overige co-auteurs wil ik bedanken voor hun gewaardeerde input en feedback die ik heb mogen ontvangen voor alle studies. Speciaal nog, Veerle Melotte en Werend Boesmans, bedankt voor jullie waardevolle input met jullie brede kennis van het ENS. Tevens, Jogchum Plat, dank voor de waardevolle inbreng die u heeft gegeven met uw brede kennis van plantsterolen.

Frank, Ralph, Sander en Zita, dank voor jullie ondersteuning. Tijdens de wekelijkse research meetings en daarbuiten hielpen jullie mij met ideën of gaven jullie waardevolle adviezen en aanvullingen. Frank, dank voor het meedenken over de leverstudies, de hulp bij de galzoutdata en het nakijken van de levermanuscripten.

Ilse, één van mijn paranimfen, samen vormden wij het team van promovendi van de kinderchirurgie en hebben wij samengewerkt aan vier van de vijf studies in dit proefschrift. Hiervan heeft de muisstudie ons de meeste grijze haren bezorgd, geloof ik. Wat kun jij ontzettend goed schrijven zeg, hier heb ik echt bewondering voor! Daarnaast is één van jouw talenten om in de krochten van de literatuur altijd wel weer een verklaring te vinden voor een of ander vraagstuk of een onderbouwing te vinden voor de bevindingen in onze studies. Samen (en met de hulp van het hele team) hebben we vier mooie artikelen gemaakt. Ik wil je ontzettend bedanken voor de fijne samenwerking die gepaard ging met flink wat gezelligheid en gegiechel. En wat hadden wij een geluk dat het deel uitmaken 
van twee afdelingen ook betekende dat we hierdoor mee mochten op dubbel zoveel leuke uitjes! Heel veel succes met de afronding van jouw promotie!

Jacqueline, Sjekkie, één van mijn paranimfen, wat waren wij een stel blije, impulsieve, luide en gniffelende geeks op één kamer. Tenminste ik denk dat gniffelen of luid hyena gehuil wel de meest passende terminologie is om ons soort lachen uit te drukken. Onze $\mathrm{pH}$-waarde daalde wel altijd bergopwaarts op de wielrenfiets, waarbij we droomden dat we ooit zulke goeie bovenbenen als "jeweetwel" zouden mogen krijgen. Toch gingen we altijd weer competitief de berg omhoog, waarbij er weer flink werd gegniffeld als we weer eens een man op een wielrenfiets hadden "gechicked". Bewonderingswaardig vind ik het dat jij je, als thriatlete, voor de fun helemaal kapot traint en je de Ironman voltooid hebt, zo knap!

Erg bedreven waren we ook met tafelvoetbal, waarbij we menig maal Wald, Steen en the Robster keihard hebben ingemaakt, ook al zullen ze het zelf niet snel toegeven. Bijna zeker dat wij met zijn allen gedeeltelijk de veroorzakers van de geluidsoverlast waren, met de nodige boze mails als gevolg. "Sorry hè!" Zo leuk dat we elkaars paranimfen zijn!

Claire (PC), Frans (Fransie) en Leontine (Leo), de eerste twee jaar van mijn promotie waren jullie mijn roomies en wat was dat gezellig! Soms zelfs zo gezellig dat we niet aan werken toekwamen. En dat was niet altijd onze eigen schuld (!), want onze kamer leek wel de koffiekamer van de chirurgie te zijn, waarbij mensen (voornamelijk Bas, David en Dennis) gedurende de hele dag langskwamen om te kletsen en koffie te drinken.

Met zijn vieren hadden we de meest rare gespreksonderwerpen, waarvan ik de onderwerpen hier niet eens durf te benoemen, of hadden we discussies over hoe films niet klopten of over themaliedjes van kinderseries uit de jaren 90. Frans en Leo: Claire en ik vinden overigens nog steeds dat jullie rare series keken, maar dat zal de generatiekloof wel zijn. Toch wel fijn dat we na ruim vier jaar discussiëren eindelijk antwoord hebben gekregen op de vraag of iemand zonder diabetes een hypo kan krijgen.

Tevens werden de nodige streken uitgehaald. In die categorie: Frans, het is je geraden dat je dat internisten naamplaatje nog steeds hebt! Dit allemaal altijd onder het genot van de meest nieuwe smaak van Tony Chocolonely, waarvan we de special edition met onze hoofden erop nog steeds niet hebben durven opeten.

Privé organiseren we nog steeds eens in de zoveel tijd een "roomie diner", waarbij om de beurt iemand moet koken en Frans zich altijd mega uitslooft met een gigantische Indische rijsttafel en unicorn (fluffy!) gekleurde pannekoeken als toetje. Geen editie wordt overgeslagen. Zo waren we zelfs tijdens een dikke sneeuwstorm naar Venlo gereden om bij Leo en Tom te gaan eten. Om de orde van grootte van deze sneeuwstorm uit te drukken: Zelfs de gigantische Dodge pick-up van Claire was hier niet tegen bestand. leder diner spelen we weer het spel 30 seconds (Claire speelt dan " 5 seconds"), maar na al die jaren staat het spelen van het spel droomtelefoon nog steeds op de to-do list! Het was een superleuke tijd met jullie en we zetten de "roomies diner" traditie voort! 
Sjekkie, Steen, Rob, en Wald, de belangrijkste vraag voor ons van de dag was altijd: gaan we lunchen of gaan we lekker lunchen? Een kleine greep uit onze gezellige gekkigheden: Zeilweekend, slutdrops (resulterend in dagenlange spierpijn), de op één shotje na uitgespeelde shotjeskaart van Basilica, biertje-balletje-bowlen, post-prandiale tafelvoetbal sessies etc. Geeks, het was me een genoegen en tot slot: "Dank u, geen moeite!"

Sebastiaan, Steen, waardige kamergenoot, ouwe Rotterdammert en de "geekste geek" van de afdeling. Wellicht dat we moeten beginnen met een verontschuldiging aan onze kantoorburen wegens geluidsoverlast. Frequent zongen (ik denk overigens dat het eerder benoemd moet worden als schreeuwen) wij namelijk mee met Paul de Leeuw met het liedje regenboog of werd random Malaa door de kamer geroepen. Muziek was wel één van de grote dingen op onze kamer met jou als top DJ van het foute uur. Zo werd iedere dag standaard begonnen met het foute uur van Q-music. Tevens waren wij vervend speler van het geluid, deden we weddenschappen om een potje bier of zaten we bijna te janken bij het bekijken van foto's van puppy's in het asiel.

Het andere geschreeuw kwam van het lachen of nadoen van de schapen-die-schreeuwenals-mensen-youtube-compilatie. Dit uitte zich uitendelijk tot "the goat dance move". Het was me een genoegen om met jou op één kamer te zitten, "jij gekke en fijne technovlinder!"

Rob, The Robster, aanstichter van de huilen-met-Rob-wielren-edities. Tochten met de nodige aantallen kilometers, hoogtemeters en steile bergen resulterend in huilende dames op wielrenfietsen (lees: sjekkie en mijzelf). En over de tocht der tochten, de alternatieve van 2017, zullen we het alsjeblieft maar niet meer hebben. Toch wist je ons altijd weer over te halen met een beloofde vlaai-eet-tussenstop. En met jouw routes leidde je ons altijd langs de mooiste stukken van Limburg en België!

In het lab waren wij de slice-buddies. Bloed, zweet en tranen hebben we in die techniek gestoken; jij voor de lever en ik voor de darm. Rob, we moeten nog steeds dat ene snode plan samen uitvoeren!

Wald, Wald-Erik, bedenker van een aantal van de bijnamen die ik had op het werk: Carla \& Juanita. Hoe je hier ook alweer opkwam, geen idee. Jij hebt op het werk en daarbuiten zo vaak Daniel Arends nagedaan dat toen we hem uiteindelijk in het echt in het theater zagen, Daniel zelve, toch bestwel tegenviel. Jouw imitaties bleken toch leuker te zijn, mede omdat je altijd daarna om jezelf zat te schuddebuiken.

Tijdens het wielrennen hoefden wij ons nooit zorgen te maken om plotseling omslaand weer, met jouw "wolk-lees-skills". Als je ooit een carrièreswitch overweegt, kun je altijd nog "weermeneer" worden (maar snap ook wel dat walvischirurg je tweede keus is). Ik denk tot slot dat we als tip mee kunnen geven aan iedere wielrenner: "Als je de weg kwijt bent, volg altijd de puppy's!"

Anne en Aurelia, jullie waren mijn roomies tijdens het laatste deel van mijn promotie. Ik geloof dat er geen dag was dat we niet allemaal kleren van Vanilia aan hadden. Het was 
altijd lachen met jullie en in onze eerste week samen op een kantoor hadden we al de meest rare gesprekken. In het nieuwe jaar (2020) werd er een sportschema op de deur gehangen waarbij we vier keer per week moesten sporten, dat was een goede motivator! Heel veel succes met jullie promotie meiden!

Alle overige lieve collega's van de chirurgie, ik ga jullie niet allemaal appart benoemen want ik heb over de jaren heen met zoveel van jullie samengewerkt. Bedankt voor jullie collegialiteit, de gezelligheid tijdens het werk en daarbuiten! Een kleine greep uit al deze gezelligheden: de nodige koffiepauzes, lunches, borrels, etentjes, Thembi middagen/avonden, lab uitjes, feestjes, Sinterklaas suprises, ambtenaren carnaval edities, wielrentochten, jachthonden wandeling etc.

Lieve collega's van de kindergeneeskunde: Charlotte, Daan, Helene, Ilse, Kimberly, Lieke, Luise, Nico, Lilian, Rob, Ruth en Teun. Wat werd ik halverwege mijn promotietraject warm door jullie ontvangen. Jullie stonden altijd klaar om te helpen of om vragen te beantwoorden. Dit team is echt een geoliede machine waarbij teamwork voorop staat. Het was altijd één brok gezelligheid tijdens het werk (en daarbuiten!) met lachen tot buikpijn aan toe. Bij elk experiment werd er wel weer een nieuw woord of rijmpje verzonnen. Daarbuiten hebben we ook de nodige gezellige activiteiten ondernomen, van borrels tot pizza, van de Efteling tot het zeer geslaagde skireisje (top organisatie Rob!). Succes met het afronden van jullie promoties!

Mijn lieve studenten: Lian, Ellen, Anja, Lotte, Tessa en Sophie, zonder jullie had ik dit allemaal niet voor elkaar gekregen! Jullie hebben me op het lab zoveel werk uit handen genomen. Mijn oneindige dank hiervoor! Naast samenwerken, kletsen en lachen (of giechelen zoals cavia's zou Bas zeggen), hoop ik dat jullie tijdens jullie stages veel geleerd hebben. Heel veel succes in de toekomst!

Lieve collega's van de intensive care in het Zuyderland, het was best spannend om na ruim vier jaar promoveren weer in de kliniek te beginnen en nog spannender om tijdens de piek van de COVID-19 uitbraak te starten. Ik wil jullie ontzettend bedanken voor de goede begeleiding, alle leermomenten, de fijne en gezellige werksfeer en de teamspirit die er heerst. Wellicht weer tot in de toekomst!

Lieve collega's van de anesthesie \& pijngeneeskunde in het MUMC+, ik wil jullie ontzettend bedanken voor jullie warme ontvangst, de tijd die jullie in me investeren om me verschillende technieken te leren, dingen uitgebreid uit te leggen en samen te reflecteren. Bedankt voor de goede begeleiding en fijne werksfeer. Ik kijk uit naar de ontwikkelingen en samenwerking de komende 5 jaar.

Lieve familie, dank voor jullie interesse, steun en alle lieve berichtjes! Jullie belangstelling en succes wensen waren een goede stimulans.

Lieve vriendinnies, kittens, koekies en sjmikkels, wat ben ik dankbaar dat wij allemaal vriendinnetjes zijn! Ik wil jullie heel erg bedanken voor de steun die jullie me hebben gegeven de afgelopen jaren. Ook dat jullie me begrepen als ik weer eens af moest zeggen, 
omdat ik weer een of andere deadline had. Dank dat ik altijd bij jullie mijn hart mocht luchten, maar vooral dat we het fijn over compleet andere dingen konden hebben, konden levelen en lekker gek konden doen onder het genot van een glaasje wijn of thee met heel veel koekjes. Dank dat jullie me onvoorwaardelijk steunen, advies geven en ontzettend aan het lachen maken. "Miauw!"

Lieve Vino, mijn Vizsla hond, mijn kleine-irritante-maar-oh-zo-lief stuk-vreten. Op het werk ben je berucht en beroemd en kregen collega's vaak (ongevraagd) foto's of filmpjes van je te zien. "Sorry not sorry!" Tijdens mijn promotie heb je voor de nodige welkome afleiding en ontspanning gezorgd. Wanneer ik thuis aan het werken was kwam je gevraagd en ook vaak ongevraagd een knuffel halen. Ik kan zo vaak lachen om de gekke dingen die je doet, je knuffelen en met je dansen door de kamer als ik weer leuke resultaten uit een analyse kreeg. Je snapt hier waarschijnlijk geen snars van. In hondentaal zou je waarschijnlijk zeggen: "woef-waf-wtf", maar ik geef je zo nog wel een koekje. Dat is voor jou toch bijna hetzelfde denk ik zo.

Lieve papa en mama, ik weet niet eens waar ik bij jullie moet beginnen. Jullie zijn mijn steun en toeverlaat en kunnen altijd zo een wijze raad geven. Jullie hebben alles mogelijk gemaakt om te staan waar ik nu sta. Ik kan jullie niet genoeg bedanken voor eigenlijk alles: de fijne en onbezorgde jeugd die jullie me hebben geven en alle mogelijkheden die jullie mij hebben geboden om te studeren en me te ontwikkelen. De drie pijlers die in mijn opvoeding (en nu nog steeds nu ik volwassen ben) altijd naar boven kwamen: doe wat je leuk vindt, volg je hart en zorg dat je (financieel) onafhankelijk bent, hebben ervoor gezorgd de persoon te worden die ik nu ben. Ook jullie gezegdes als: "plannen is vooruitzien" en "een slimme meid is op alles voorbereid", hebben mij ver geholpen. Jullie steunen me onvoorwaardelijk en tijdens onze brainstormsessies weten jullie er altijd weer voor te zorgen dat ik er zelf achter kom wat ik nou eigenlijk wil (terwijl jullie dat natuurlijk allang wisten, want jullie kennen me te goed). "Pappie en moemoe ik houd van jullie! Knuffels van de knuffel".

Lieve Timo, allerliefste "apie/knorbert", of beter bekend op het werk als "der Timo". Jij ging altijd (als mijn bodyguard) midden in de nacht met me mee naar de uni voor de darmslice-experimenten, omdat ik anders bang was alleen in het donker rond te lopen op de uitgestorven uni. Even voor de goede orde: het ging hier om tijdstippen als 03:00 uur 's nachts of elk ander onzondags tijdstip. Ik bedoel: hoeveel best-boyfriend-in-the-worldpunten wil je nog meer scoren?

Thuis zorg je er altijd voor om me uit de werksfeer te halen, "geeken we around", kook je superlekker voor me of gaan we gezellig samen uit eten. Daarnaast vind ik het juist heel fijn om het met jou over heel andere dingen te hebben dan mijn werk. Jij weet er altijd op de juiste momenten voor me te zijn, me te pushen wanneer nodig, maar (heel belangrijk) je weet me ook af te remmen als ik weer eens doordraaf met werken. Je weet hoe gek en trots ik op jou ben! "Knorrie, I love you to the moooooon!" 

List of publications 



\section{List of publications}

\section{This thesis}

C. Heymans, I.H. de Lange, M.C. Hütten, K. Lenaerts, N.J.E. de Ruijter, L.C.G.A. Kessels, G. Rademakers, V. Melotte, W. Boesmans, M. Saito, H. Usuda, S.J. Stock, O.B. Spiller, M.S. Payne, B.W. Kramer, J.P Newnham, A.H. Jobe, M.W. Kemp, W.G. van Gemert, T.G.A.M. Wolfs. Chronic Intra-Uterine Ureaplasma parvum Infection Induces Injury of the Enteric Nervous System in Ovine Fetuses. Front Immunol. 2020 Mar 17;11:189

C. Heymans, L.R. Heij, K. Lenaerts, M. den Dulk, M. Hadfoune, C. van Heugten, O.B. Spiller, M.L. Beeton, S.J. Stock, A.H. Jobe, M.S. Payne, M.W. Kemp, B.W. Kramer, J. Plat, W.G. van Gemert, T.G.A.M. Wolfs. Prophylactic Intra-Uterine $\beta$-Cyclodextrin Administration during Intra-Uterine Ureaplasma Parvum Infection Partly Prevents Liver Inflammation without Interfering with the Enterohepatic Circulation of the Fetal Sheep. Nutrients. 2020 May;12(5)

C. Heymans*, I.H. de Lange* , K. Lenaerts, L.C.G.A. Kessels, M. Hadfoune, G. Rademakers, V. Melotte, W. Boesmans, B.W. Kramer, A.H. Jobe, M.W. Kemp, W.G. van Gemert, T.G.A.M. Wolfs. Chorioamnionitis induces enteric nervous system injury: effects of timing and inflammation in the ovine fetus. Molecular Medicine. 2020 Sept;26(1)

* Contributed equally

C. Heymans, M. den Dulk, K. Lenaerts, L.R. Heij, I.H. de Lange, M. Hadfoune, C. van Heugten, B.W. Kramer, A.H. Jobe, M. Saito, M.W. Kemp, T.G.A.M. Wolfs, W.G. van Gemert. Chorioamnionitis induces hepatic inflammation and time-dependent changes of the enterohepatic circulation in the ovine fetus. Sci Rep. 2021 May 14;11(1):10331

\section{Other}

C. Heymans, J. van Bastelaar, R.G.J. Visschers, Y.L.J. Vissers. Sentinel Node Procedure Obsolete in Lumpectomy for Ductal Carcinoma In Situ. Clin Breast Cancer. 2017 Jun;17(3)

R.W.Y.Granzier, J.van Bastelaar, S.M.J.van Kuijk, K.F.H.Hintzen, C.Heymans, L.L.B.Theunissen, E.R.M. van Haaren, A. Janssen, G.L. Beets, Y.L.J. Vissers. Reducing seroma formation and its sequelae after mastectomy by closure of the dead space: The interim analysis of a multi-center, double-blind randomized controlled trial (SAM trial). Breast. 2019 Aug;46:81-86

L. de Rooij, S. M. J. van Kuijk, R. W.Y. Granzier, K. F. H. Hintzen, C. Heymans, L. L. B. Theunissen, E. M. von Meyenfeldt, J. A. van Essen, E. R. M. van Haaren, A. Janssen, Y. L. J. Vissers, G. L. Beets, J. van Bastelaar. Reducing Seroma Formation and Its Sequelae After Mastectomy by Closure of the Dead Space: A Multi-center, Double-Blind Randomized Controlled Trial (SAMTrial). Ann Surg Oncol. 2020 Oct 19 

Curriculum Vitae 



\section{Curriculum Vitae}

Cathelijne Heymans was born on the $30^{\text {th }}$ of November 1990 in Zoetermeer, the Netherlands. After finishing the first year of high school at the Alfrink College in Zoetermeer, she and her parents moved to the south of Limburg where she graduated from the Sint-Maartenscollege in Maastricht in 2009. Afterwards, Cathelijne started her medical training at the Faculty of Health, Medicine and Life Sciences at Maastricht University. During her studies, she taught resuscitation courses (Taskforce QRS) and was a member of the organization committee of the extracurricular course on resuscitation medicine. After graduating in July 2015 , she started her first job as a surgical resident (ANIOS) at the Department of Surgery at the Erasmus Medical Center Rotterdam.

In February 2016, Cathelijne started as a PhD candidate at the Department of Surgery within NUTRIM School of Nutrition and Translational Research in Metabolism at Maastricht University under the supervision of prof. dr. W.G. van Gemert (pediatric surgery, Maastricht University Medical Center +), dr. T.G.A.M. Wolfs (pediatrics, Maastricht University) and dr. K. Lenaerts (surgery, Maastricht University). During her PhD, she investigated the effect of chorioamnionitis and perinatal stress on the enteric nervous system, liver and enterohepatic circulation of bile acids, and evaluated a therapeutic intervention to prevent hepatic inflammation.

In April 2020, Cathelijne continued her career as a resident (ANIOS) at the Intensive Care Unit at Zuyderland Medical Center Heerlen/Sittard-Geleen. As of June 2021, she started her anesthesiology residency (AIOS) at the Maastricht University Medical Center +.

Cathelijne is living together with her boyfriend, Timo Reichelt. They are proud "dog parents" of their Vizsla, Vino (October 2018). 
